University of Tennessee Health Science Center

UTHSC Digital Commons

\title{
The Role of BiP Co-chaperone SIL1 in Marinesco-Sjögren Syndrome Pathogenesis
}

\author{
Viraj Paresh Ichhaporia \\ University of Tennessee Health Science Center
}

Follow this and additional works at: https://dc.uthsc.edu/dissertations

Part of the Medical Cell Biology Commons, and the Medical Molecular Biology Commons

\section{Recommended Citation}

Ichhaporia, Viraj Paresh (http://orcid.org/0000-0001-7466-6487), "The Role of BiP Co-chaperone SIL1 in Marinesco-Sjögren Syndrome Pathogenesis" (2018). Theses and Dissertations (ETD). Paper 469.

http://dx.doi.org/10.21007/etd.cghs.2018.0470.

This Dissertation is brought to you for free and open access by the College of Graduate Health Sciences at UTHSC Digital Commons. It has been accepted for inclusion in Theses and Dissertations (ETD) by an authorized administrator of UTHSC Digital Commons. For more information, please contact jwelch30@uthsc.edu. 


\title{
The Role of BiP Co-chaperone SIL1 in Marinesco-Sjögren Syndrome Pathogenesis
}

\begin{abstract}
Marinesco-Sjögren syndrome (MSS) is a rare, autosomal recessive, multisystem disorder, which is characterized by cerebellar ataxia, early-onset bilateral cataracts, and progressive myopathy amongst other symptoms. MSS has been attributed to mutations in the SIL1 gene, which encodes a nucleotide exchange factor for the endoplasmicreticulum- resident Hsp70 chaperone, BiP. To date, there are 46 MSSassociated mutations that have been reported in SIL1, which occur throughout this gene and are predicted to result in a loss of SIL1's function. The large majority of these mutations cause deletions of large fractions of the SIL1 protein. Nine MSS-associated mutations are particularly interesting because they alter less than six amino acids, yet are associated with a phenotype indistinguishable from a near-full length deletion of SIL1. The mechanisms by which these nine mutations lead to a loss of SIL1's function are not well understood. On the other hand, it remains unclear how the loss of SIL1 leads to the multisystem defects observed in MSS, selectively affecting certain tissues while sparing others. Our goal was to answer these two questions.

We have shown that the selected nine MSS-associated SIL1 mutations may dramatically alter the protein microenvironment and disrupt intramolecular interactions, such that it alters the folding properties of SIL1 and renders it aggregation-prone. This offers a potential mechanism by which mutations in SIL1 cause a loss of its function. We validated that the C57BL/6 Sil1Gt mouse model, which harbors a genetic disruption of Sil1, phenocopies numerous aspects of the MSS-phenotype and represents a valid preclinical model system to investigate the MSS-associated pathology and explore pharmacotherapeutic strategies. Using a combination of the Sil1Gt mice and SIL1- deficient MSS-patient-derived lymphoblastoid cell lines, we explored the biosynthesis and secretion of immunoglobulins (Ig), which are the best characterized substrate of BiP to date. In vivo antigen-specific immunizations and ex vivo LPS stimulation of splenic B cells revealed that the Sil $1 \mathrm{Gt}$ mouse was indistinguishable from wild-type agematched controls, in terms of both the kinetics and magnitude of antigen-specific antibody responses. There was no significant accumulation of BiP-associated Ig assembly intermediates or evidence that another molecular chaperone system was used for antibody production in the LPS-stimulated splenic B cells from Sil1Gt mice. ER chaperones were expressed at the same level in wild-type and Sil1Gt mice, indicating that there was no evident compensation for the disruption of Sil1. These results were confirmed and extended in lymphoblastoid cell lines from individuals with MSS, leading us to conclude that, surprisingly, the SIL1 was dispensable for antibody production.
\end{abstract}

Using Sil1Gt mice, we next characterized the molecular aspects of progressive myopathy associated with MSS. Proteomic-profiling of quadriceps at the onset of myopathy revealed that SIL1 deficiency affected multiple pathways critical to muscle physiology. We observed an increase in ER chaperones prior to the onset of muscle weakness, which was complemented by up-regulation of multiple protein degradation pathways. These responses were inadequate to maintain normal expression of secretory pathway proteins, including insulin and IGF-1 receptors. There was a paradoxical downstream PI3K-AKT signaling and glucose uptake in Sil1-disrupted skeletal muscles, all of which were insufficient to maintain systemic glucose homeostasis and muscle mass. Together, these data reveal defects in maintaining ER homeostasis upon SIL1 loss, which are countered by multiple compensatory responses that are ultimately unsuccessful, leading to trans-organellar proteostasis collapse and myopathy.

\section{Document Type}

Dissertation 


\section{Degree Name}

Doctor of Philosophy (PhD)

\section{Program}

Biomedical Sciences

\section{Research Advisor}

Linda M. Hendershot, Ph.D.

\section{Keywords}

BiP/GRP78/HSPA5, Chaperones, Endoplasmic Reticulum, Marinesco-Sjögren syndrome, Muscle biology, SIL1/BAP

\section{Subject Categories}

Medical Cell Biology | Medical Molecular Biology | Medical Sciences | Medicine and Health Sciences 
The Role of BiP Co-chaperone SIL1 in Marinesco-Sjögren Syndrome Pathogenesis

\author{
A Dissertation \\ Presented for \\ The Graduate Studies Council \\ The University of Tennessee \\ Health Science Center
}

\author{
In Partial Fulfillment \\ Of the Requirements for the Degree \\ Doctor of Philosophy \\ From The University of Tennessee
}

By

Viraj Paresh Ichhaporia

December 2018 
Chapter 2 (C) 2015 by Viraj P. Ichhaporia et al. All other material (C) 2018 by Viraj P. Ichhaporia. All rights reserved. 


\section{DEDICATION}

Dedicated to my parents and my grandparents. It is because of their countless sacrifices, unconditional love, and everlasting support that have inspired me to push beyond my personal limits, and who have instilled in me the values that I most cherish today. 


\section{ACKNOWLEDGEMENTS}

It has been an incredible journey over the past seven years, studded with numerous memories and learning lessons, that brings me to the end of my dissertation. Right from my lab rotation to until now, I have never stopped learning from Dr. Linda M. Hendershot, my Ph.D. advisor, and I am indebted to her for that. Dr. Hendershot has been an excellent mentor over these years and is responsible for inculcating in me the core values of an ideal scientist, while always being supportive and enabling me to be a better version of myself, at a professional and a personal level. I am grateful to her for giving me the opportunity to be a part of the family at Hendershot laboratory and the cherished memories that I carry forward. I shall always remember what I have learnt here.

Along with Dr. Hendershot, I am very thankful to Melissa Mann for teaching me the rigor and prowess with which scientific experimentation should be performed. It is Ms. Mann who taught me many of the lab techniques that I proudly claim as my 'bread and butter' skills. I also wish to thank her for her help with numerous aspects of my dissertation research. I am also thankful to my friends within the Hendershot lab, both past and present, for enlivening the work place and for always being supportive, encouraging, and helpful at any given opportunity. It is only because of them that the late nights and long hours passed by with ease. I thank Tyler Sanford for early discussions on the potential of this project and helping me transition into this research field. I thank Drs. Brian Murphy and Walid Awad for their assistance with managing the mouse colony and genotyping. I truly appreciate the support and constructive feedback I have received over the years from Dr. Kyung Tae Chung (who discovered mammalian SIL1), Dr. Joel Otero, Dr. Ethel Pereira, Dr. Matthias Feige, Dr. Julia Behnke, Dr. Jyoti Sinha, Dr. Greg Poet, Dr. Kristine Pobre, Dr. Amanda Preston, and Mary Carson Irvine. A special thanks to my friends and fellow graduate students, Christina Oikonomou and Rachael Wood, for their wholehearted support. I wish them the best for their own Ph.D. journeys. I was fortunate to work with an excellent summer student and a budding young scientist, Jessica Joyce, and thank her for her help with the SIL1 mutants project.

I sincerely also thank my committee members, Dr. Alessandra d'Azzo, Dr. Tony Marion, Dr. David Nelson, Dr. J. Paul Taylor, and Dr. Stanislav Zakharenko, for their time, advice, guidance, and support over these years. The decision to pursue proteomics and many riveting answers stemmed from the scientific discussions during my committee meetings. I would like to thank Dr. Alessandra d'Azzo, Dr. Mondira Kundu, Dr. J. Paul Taylor, and Dr. Bo Wang for their generous gift of reagents, and Dr. Diantha Van de Vlekkert and Dr. Cath Drummond for their help with establishing myoblast cultures.

Without the help from my collaborators, answering questions using technologies that lie beyond my expertise would not have been possible. In doing so, these collaborators not only offered me their time, but also excellent opportunities to educate myself and understand complex data in a meaningful manner. I thank Sharon Frase and Linda Horner for their assistance with electron microscopy studies; Dr. Peter Vogel, Sean 
Savage, Michael Anderson, Michael Straign, and Chandra Savage for their help animal pathology and pharmacokinetic studies; Dr. Junmin Peng, Dr. Vishwajeeth Pagala, Dr. Kanisha Kavdia, and Dr. Yong-Dong Wang for their help with proteomic studies; Dr. Jieun Kim and Dr. Walter Akers for their help with PET- $\mu$ CT studies; Dr. Chunliang Li and Shaela Wright for making the CRISPR-mediated SIL1 knockout cell lines a possibility; Yumei Zhang for all her help with the structural analysis of the SIL1 mutants, and Dr. Fabio Demontis for providing critical feedback and improving our SIL1myopathy article.

I would like to acknowledge the funding support I have received from UTHSC and St. Jude in the form of travel grants that have enabled me to present my research on diverse platforms and engage in career development activities. For this, I thank Dr. Donald Thomason, Dr. Gerard Zambetti, and Dr. Isaac Donkor. I thank NIH and ALSAC for their funding support through Dr. Hendershot that has allowed me to perform my experimentation. I also thank the UTHSC deans along with the past and present members of the Graduate Student Executive Committee for empowering and supporting graduate students.

I also appreciate the help I have received from the faculty and staff members at UTHSC and St. Jude. In particular, I would like to call out the current and past IBS Program Directors, Dr. Elizabeth Fitzpatrick, Dr. Rennolds Ostrom, and Dr. Pat Ryan; and Ms. Elizabeth Webb, Late Ms. Tricia Satkowski, and Ms. Denise Slaughter, our past and present program coordinators from UTHSC and St. Jude. I also thank the faculty members of the Microbiology, Biochemistry, and Immunology department, and my colleagues within the Tumor Cell Biology department for their scientific feedback and guidance.

Lastly, I am grateful beyond measure to my family, especially my parents, without whom this life-changing journey would have never materialized, and for their ever-lasting support and inspiration. I am very thankful to my fiancé for being my northern star and my strength. Last, but certainly not the least, I would like to end by thanking my friends who made my time in Memphis a truly enjoyable and nostalgic experience. 


\begin{abstract}
Marinesco-Sjögren syndrome (MSS) is a rare, autosomal recessive, multisystem disorder, which is characterized by cerebellar ataxia, early-onset bilateral cataracts, and progressive myopathy amongst other symptoms. MSS has been attributed to mutations in the SIL1 gene, which encodes a nucleotide exchange factor for the endoplasmicreticulum-resident Hsp70 chaperone, BiP. To date, there are $46 \mathrm{MSS}$-associated mutations that have been reported in SIL1, which occur throughout this gene and are predicted to result in a loss of SIL1's function. The large majority of these mutations cause deletions of large fractions of the SIL1 protein. Nine MSS-associated mutations are particularly interesting because they alter less than six amino acids, yet are associated with a phenotype indistinguishable from a near-full length deletion of SIL1. The mechanisms by which these nine mutations lead to a loss of SIL1's function are not well understood. On the other hand, it remains unclear how the loss of SIL1 leads to the multisystem defects observed in MSS, selectively affecting certain tissues while sparing others. Our goal was to answer these two questions.
\end{abstract}

We have shown that the selected nine MSS-associated SIL1 mutations may dramatically alter the protein microenvironment and disrupt intramolecular interactions, such that it alters the folding properties of SIL1 and renders it aggregation-prone. This offers a potential mechanism by which mutations in SIL 1 cause a loss of its function. We validated that the C57BL/6 Sill ${ }^{\mathrm{Gt}}$ mouse model, which harbors a genetic disruption of Sill, phenocopies numerous aspects of the MSS-phenotype and represents a valid preclinical model system to investigate the MSS-associated pathology and explore pharmacotherapeutic strategies. Using a combination of the Sill ${ }^{\mathrm{Gt}}$ mice and SIL1deficient MSS-patient-derived lymphoblastoid cell lines, we explored the biosynthesis and secretion of immunoglobulins (Ig), which are the best characterized substrate of BiP to date. In vivo antigen-specific immunizations and ex vivo LPS stimulation of splenic B cells revealed that the Sill $^{\text {Gt }}$ mouse was indistinguishable from wild-type age-matched controls, in terms of both the kinetics and magnitude of antigen-specific antibody responses. There was no significant accumulation of BiP-associated Ig assembly intermediates or evidence that another molecular chaperone system was used for antibody production in the LPS-stimulated splenic B cells from Sill ${ }^{\mathrm{Gt}}$ mice. ER chaperones were expressed at the same level in wild-type and Sill ${ }^{\mathrm{Gt}}$ mice, indicating that there was no evident compensation for the disruption of Sill. These results were confirmed and extended in lymphoblastoid cell lines from individuals with MSS, leading us to conclude that, surprisingly, the SIL1 was dispensable for antibody production.

Using Sil1 $1^{\mathrm{Gt}}$ mice, we next characterized the molecular aspects of progressive myopathy associated with MSS. Proteomic-profiling of quadriceps at the onset of myopathy revealed that SIL1 deficiency affected multiple pathways critical to muscle physiology. We observed an increase in ER chaperones prior to the onset of muscle weakness, which was complemented by up-regulation of multiple protein degradation pathways. These responses were inadequate to maintain normal expression of secretory pathway proteins, including insulin and IGF-1 receptors. There was a paradoxical 
enhancement of downstream PI3K-AKT signaling and glucose uptake in Sill-disrupted skeletal muscles, all of which were insufficient to maintain systemic glucose homeostasis and muscle mass. Together, these data reveal defects in maintaining ER homeostasis upon SIL1 loss, which are countered by multiple compensatory responses that are ultimately unsuccessful, leading to trans-organellar proteostasis collapse and myopathy. 


\section{TABLE OF CONTENTS}

CHAPTER 1. INTRODUCTION ....................................................................................

Biosynthesis of Secretory Pathway Proteins - Journey into the ER................................1

Protein Folding and Quality Control in the Endoplasmic Reticulum ..............................3

Chaperone Families of the Endoplasmic Reticulum....................................................

Lectin chaperones and co-chaperones...................................................................

The HSP70 chaperone BiP/Grp78: It's ATPase cycle and its co-chaperone

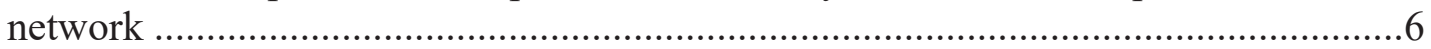

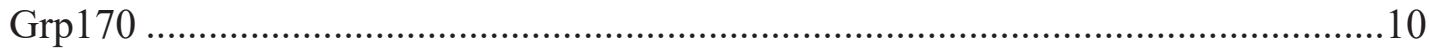

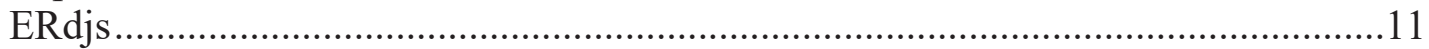

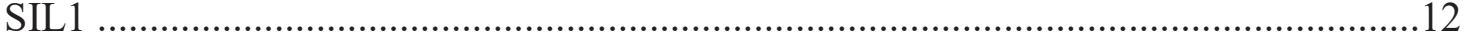

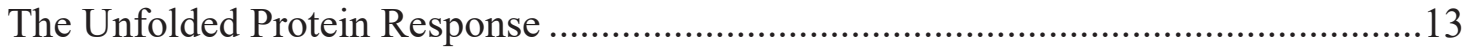

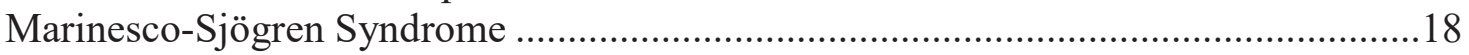

CHAPTER 2. SIL1, A NUCLEOTIDE EXCHANGE FACTOR FOR BIP, IS NOT REQUIRED FOR ANTIBODY ASSEMBLY OR SECRETION.......................24

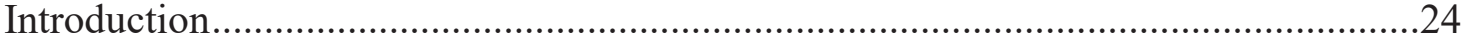

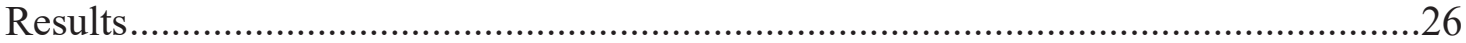

Detection of disrupted Sill transcripts in woozy mice .............................................26

Characterization of immune populations in wild-type and woozy mice ...................26

$\mathrm{Sill}^{\mathrm{Gt}}$ mice produce normal levels of antigen-specific antibodies in response to immunization with a T-dependent antigen...............................................................29

LPS-stimulated B cells from Sill ${ }^{\mathrm{Gt}}$ mice synthesize and secrete IgM antibodies

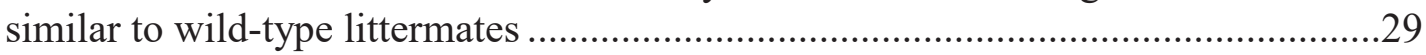

Sil1 disruption does not affect chaperone levels in LPS stimulated B cells ...............32

Immunoglobulin synthesis is not relegated to a different chaperone system in

SIL1 disrupted splenic B cells during differentiation .................................................35

Antibody assembly and secretion are normal in lymphoblastoid lines from

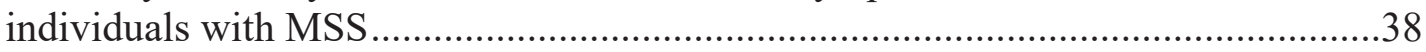

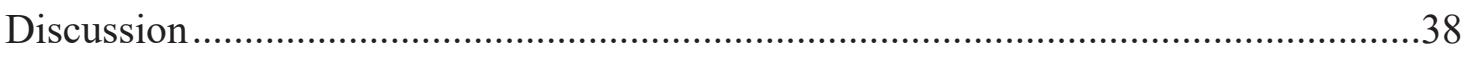

SIL1 is not required for Ig assembly or secretion....................................................38

How is BiP released from Ig subunits to allow their assembly when SIL1 is absent?

Why do SIL1 disrupted mice and MSS patients have defects that only appear to

affect some systems? .............................................................................................4

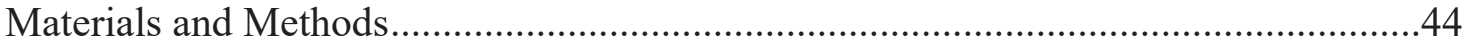

Generation of wild-type and Sill-disrupted mice ………........................................4

Detection of mutant transcripts and protein in woozy mice ........................................4

Characterization of immune cell populations in spleen and thymus ...........................45

Immunization protocol and ELISA for antigen-specific response .............................45

Ex vivo stimulation of purified splenocytes with LPS ...........................................45

EBV-transformed lymphoblastoid cell lines ............................................................46

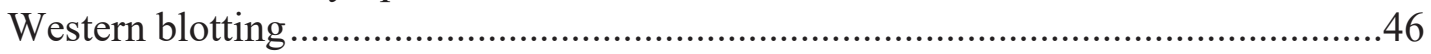

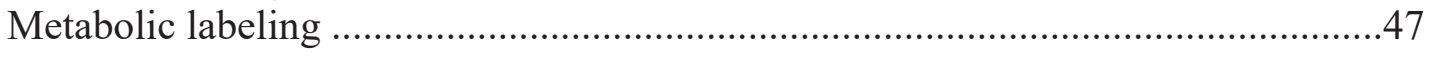


Sill sequencing

\section{CHAPTER 3. SIL1, THE ENDOPLASMIC-RETICULUM-LOCALIZED BIP CO-CHAPERONE, PLAYS A CRITICAL ROLE IN MAINTAINING SKELETAL MUSCLE PROTEOSTASIS AND PHYSIOLOGY}

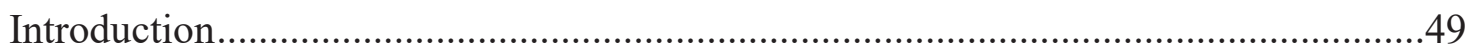

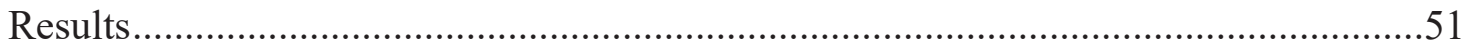

Sill $1^{\mathrm{Gt}}$ mice display evidence of a progressive myogenic myopathy ...........................51

SIL1 disruption leads to widespread changes in the quadriceps proteome .................58

Loss of SIL1 disrupts ER homeostasis as mice age ...................................................61

Evidence for inadequate compensation by cellular degradation systems ...................64

Disruption of SIL1 has profound effects on components of the insulin signaling

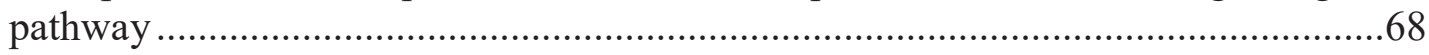

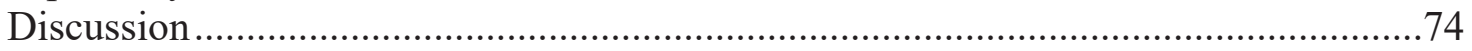

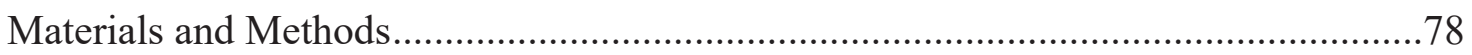

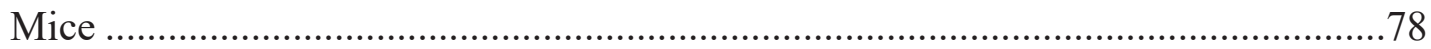

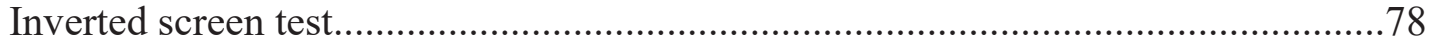

Histopathology and immunohistochemistry ……………......................................79

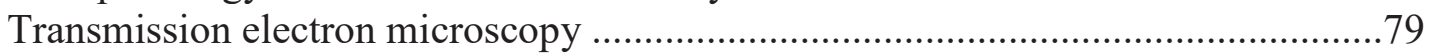

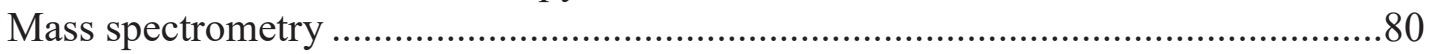

Protein digestion and peptide isobaric labeling by Tandem Mass Tags (TMTs) .80

Two dimensional HPLC and mass spectrometry ................................................ 80

Mass spectrometry data analysis and bioinformatics.......................................... 80

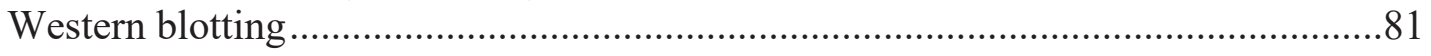

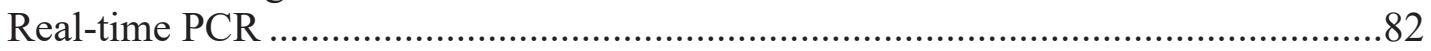

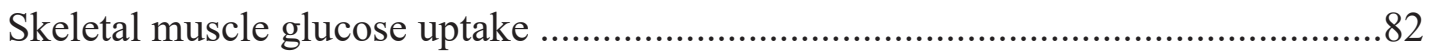

\section{CHAPTER 4. MSS-ASSOCIATED MUTATIONS DESTABILIZE SIL1 AND} CAN CAUSE MARINESCO-SJÖGREN SYNDROME.........................................84

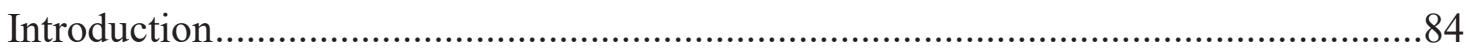

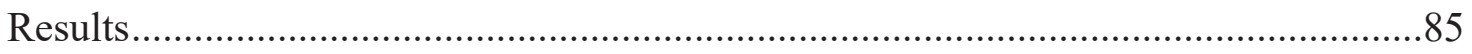

MSS-associated SIL1 mutations affect highly conserved regions, and in most cases are predicted to disrupt regional alpha-helicity ................................................. 85

Structural modeling suggests that the selected MSS-associated mutations affect residues which play a key role in maintaining SIL1's structural integrity .................90

MSS-associated SIL1 mutants form NP40-insoluble aggregates ................................98

MSS-associated SIL1 mutants alter its half-life …………......................................100

Discussion and Future Direction.............................................................................. 100

Completing studies on the half-lives of SIL1 mutants.............................................102

Do the selected SIL1 mutants display a dominant-negative behavior? ....................102

Evaluating the ability of chemical chaperones to rescue SIL1 mutants ...................102

Investigating the ability of SIL1 mutants to bind BiP .............................................103

Investigating the NEF activity of corrected SIL1 mutants .......................................104

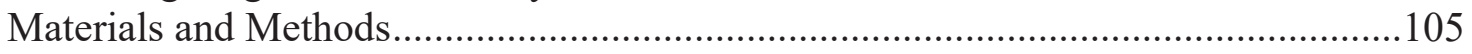

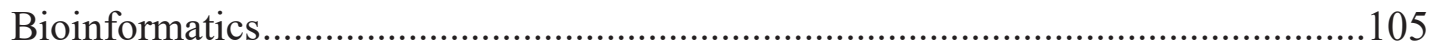


Multiple sequence alignment ................................................................................ 105

Prediction of functional effects of missense SIL1 mutations .............................. 105

Prediction of alpha-helical and aggregation propensities using TANGO .......... 105

Analysis of the yeast Sil1p and human HSPBP1 structures ......................................106

Generation of MSS-associated SIL1 mutants using site-directed mutagenesis .......106

Cell culture, transfections, western blotting, and densitometric quantitations ........108

CHAPTER 5. DISCUSSION ...............................................................................110

Molecular Mechanisms Underlying Marinesco-Sjögren Syndrome ...........................110

SIL1 mutations and the genetic heterogeneity associated with MSS ........................110

The neurological features of MSS ...................................................................111

The myopathy in MSS ...................................................................................111

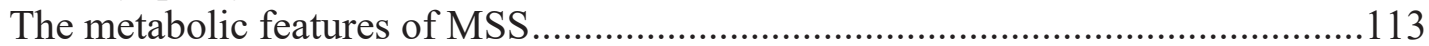

Differences Between the Two Murine Models of MSS ………….............................114

Why Does the Loss of SIL1 in Humans and Mice Selectively Affect Some Tissues,

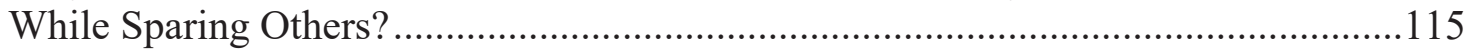

The SIL1 expression-effect paradox ..................................................................115

Possibilities for selective vulnerability of certain tissues in MSS ...........................115

An unexpected new role for SIL1 as a BiP-reductase ..........................................116

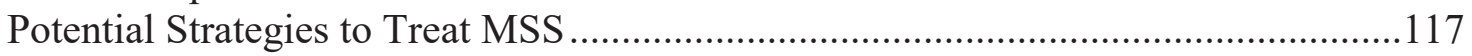

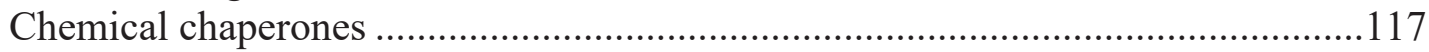

Modulation of the PERK pathway ....................................................................118

The Role of SIL1 in the Pathogenesis of Other Diseases ...........................................119

SIL1 and neurodegenerative diseases ...............................................................119

SIL1 and gliomas, the most common central nervous system tumors......................119

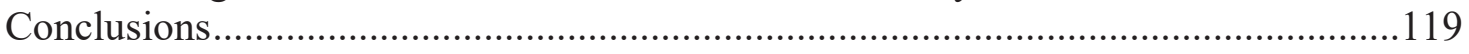

LIST OF REFERENCES ............................................................................................121

APPENDIX. GLUCOSE HANDLING DEFECTS IN SIL1 ${ }^{\text {GT }}$ MICE AND

GENERATION OF A CRISPR/CAS9-MEDIATED SIL1-NULL MODEL

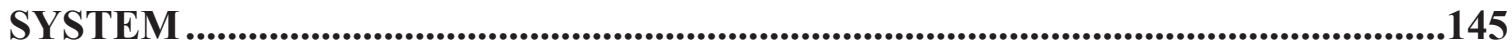

VITA..............................................................................................................................149 


\section{LIST OF TABLES}

Table 4-1. MSS-associated SIL1 mutations that alter less than 6 amino acids. .86

Table 4-2. Sillp and HSPBP1 amino acid residues corresponding to MSSassociated human SIL1 mutants.

Table 4-3. Primer sequences for the generation of SIL1 mutants using site-directed mutagenesis. 


\section{LIST OF FIGURES}

Figure 1-1. The free energy landscape during protein folding and aggregation. .............2

Figure 1-2. The ER protein quality control machinery.............................................5

Figure 1-3. The ATPase cycle of BiP and the co-chaperones regulating it.....................

Figure 1-4. Structure of Sil1 and Sil1 complexed with BiP's NBD............................ 14

Figure 1-5. Differences in the mechanisms of nucleotide exchange by Sill and Grp170, the two ER-resident NEFs of BiP. .............................................15

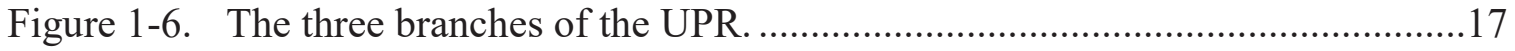

Figure 1-7. The relative frequencies of signs and symptoms associated with MSS.......19

Figure 1-8. Genetic disruption of murine Sil1 phenocopies MSS symptoms.................21

Figure 2-1. Schemes for quantifying SIL1 mRNA and genotyping mice. ....................27

Figure 2-2. Disruption of Sill gene does not affect splenocyte or thymocyte

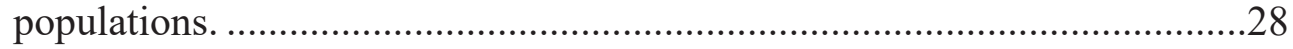

Figure 2-3. Sill ${ }^{\mathrm{Gt}}$ mice respond to T-dependent antigens similarly to age-matched Sil1 ${ }^{\mathrm{WT}}$ mice.

Figure 2-4. Disruption of Sill gene does not adversely affect IgM biosynthesis or secretion in response to ex vivo LPS stimulation. ........................................31

Figure 2-5. Disruption of Sill does not increase Ig heavy or light chain aggregation. ...33

Figure 2-6. Chaperone levels are unaltered during plasmablast differentiation in Sill ${ }^{\mathrm{Gt}}$ mice.

Figure 2-7. Loss of SIL1 does not redirect IgM assembly to another chaperone system.

Figure 2-8. Disruption of SIL1 does not alter BiP's interaction with the Ig heavy chain.

Figure 2-9. Characterization of Lymphoblastoid cell lines from normal controls and MSS affected individuals.

Figure 2-10. Disruption of Sill gene in MSS-patient-derived LBLs does not adversely affect the levels of ER chaperones. 
Figure 2-11. B Lymphoblastoid cell lines produced from individuals with MarinescoSjögren syndrome synthesize and secrete $\operatorname{IgG}$ antibodies normally.

Figure 3-1. SIL1 is required to maintain muscle strength and mass as mice age............52

Figure 3-2. Loss of SIL1 predominantly affects glycolytic skeletal muscles. 53

Figure 3-3. The myopathy resulting from SIL1 loss predominantly affects fast myofibers.....

Figure 3-4. Sill ${ }^{\mathrm{Gt}}$ skeletal muscles display characteristic myopathic signatures. 55

Figure 3-5. SIL1 disruption perturbs all major cellular organelles and pathways critical for normal quadriceps function.

Figure 3-6. Proteomic study reveals that loss of SIL1 affects pathways critical for normal muscle physiology.

Figure 3-7. SIL1 is required to maintain ER proteostasis in quadriceps as mice age. ....62

Figure 3-8. Loss of SIL1 leads to activation of the Unfolded Protein Response. .63

Figure 3-9. Loss of SIL1 leads to disruption of ER proteostasis. .65

Figure 3-10. Loss of SIL1 results in compensating increases in all cellular protein degradation systems. .66

Figure 3-11. Loss of SIL1 leads to aggregation of cytosolic small heat shock chaperones and disruption of cytosolic proteostasis.

Figure 3-12. Loss of SIL1 leads to aggregation of cytosolic small heat shock chaperones and disruption of cytosolic proteostasis.

Figure 3-13. Loss of SIL1 has profound effects on IR signaling. 71

Figure 3-14. Loss of SIL1 leads to basal activation of growth factor signaling. . .72

Figure 3-15. Evaluating the expression of genes involved in skeletal muscle regeneration, atrophy, and glucose uptake.

Figure 3-16. Schematic model describing the molecular responses to SIL1 loss in skeletal muscles leading to myopathy.

Figure 4-1. Schematic of the human SIL1 protein indicating the known MSSassociated missense mutations and small in-frame deletions.

Figure 4-2. Alignment of the SIL1 protein sequence across multiple species. . .88

Figure 4-3. Predicted detrimental effects of point mutations in SIL1. .89 
Figure 4-4. TANGO-AGADIR predicted alpha-helix forming propensities of the selected SIL1 mutant.

Figure 4-5. TANGO-predicted aggregation propensity of the region affected by the p.R92W point mutation in SIL1.

Figure 4-6. The interaction of Sil1p and HSPBP1 with their respective HSP70 chaperones.

Figure 4-7. Structural mapping of the residues corresponding to p.L206Q, p.V231_I232del, and p.G312E/R. .95

Figure 4-8. Structural mapping of the residues corresponding to p.R298C and $\triangle \mathrm{RYRQD}$.

Figure 4-9. MSS-associated SIL1 mutants form NP40-insoluble aggregates.

Figure 4-10. SIL1 mutations alter its stability and degradation kinetics. 101

Figure A-1. Sill ${ }^{\mathrm{Gt}}$ mice demonstrate glucose intolerance and insulin resistance. 145

Figure A-2. Loss of SIL1 leads to defects in brain glucose uptake in Sil1 ${ }^{\mathrm{Gt}}$ mice 146

Figure A-3. Deep sequencing reveals successful CRISPR/Cas9-mediated targeting of the Sill locus in murine $\mathrm{C} 2 \mathrm{C} 12$ myoblasts.

Figure A-4. SIL1-null myoblasts retain the ability to differentiate into myotubes and demonstrate molecular changes similar to that observed in Sill ${ }^{\mathrm{Gt}}$ skeletal muscles. 


\section{LIST OF ABBREVIATIONS}

$\% \mathrm{ID} / \mathrm{g}$

AARS

ACC

APC

APO

ARM

ATF6

ATP

BBB

$\mathrm{BiP}$

BiP 44k

bZIP

CCFDN

CHOP

CHX

CNX

COPII

CREB

CRT

Ctsh

Ctsl
Percentage Injected Dose per Gram Body Weight

Amino-Acyl tRNA Synthetase

Acetyl-CoA Carboxylase

Allophycocyanin

Apolipoprotein

Armadillo-Like Repeats

Activating Transcription Factor 6

Adenosine Triphosphate

Blood-brain Barrier

Immunoglobulin binding protein

Nucleotide binding domain of BiP of $44 \mathrm{kDa}$

Basic Leucine Zipper

Congenital Cataracts, Facial Dysmorphism, and Neuropathy Syndrome

C/EBP Homologous Protein

Cycloheximide

Calnexin

Coatamer protein II

cAMP-Responsive Element-Binding

Calreticulin

Cathepsin $\mathrm{H}$

Cathepsin L 


\begin{tabular}{|c|c|}
\hline DSP & Dithiobis Succinimidyl Propionate \\
\hline DTT & Dithiothreitol \\
\hline EBV & Epstein-Barr Virus \\
\hline EDEM & ER Degradation Enhancing $\alpha$-Mannosidase-Like Protein \\
\hline $\mathrm{eIF} 2 \alpha$ & Eukaryotic Initiation Factor $2 \alpha$ \\
\hline EM & Electron Microscopy \\
\hline ER & Endoplasmic Reticulum \\
\hline ERAD & Endoplasmic-Reticulum-Associated Degradation \\
\hline ERDJs & ER-Resident DnaJ-Like Proteins \\
\hline ERQC & Endoplasmic Reticulum Protein Quality Control \\
\hline ERS & Endoplasmic Reticulum Stress \\
\hline ERSE & ER Stress Response Elements \\
\hline FDA & Food and Drug Administration \\
\hline GADD34 & Growth Arrest And DNA-Damage-Inducible Protein 34 \\
\hline gDNA & Genomic DNA \\
\hline GET & Guided Entry of TA \\
\hline GO & Gene Ontology \\
\hline GRP & Glucose Regulated Protein \\
\hline GSEA & Gene-Set Enrichment Analysis \\
\hline $\mathrm{H} \& \mathrm{E}$ & Hematoxylin and Eosin \\
\hline $\mathrm{HC}$ & Immunoglobulin Heavy Chain \\
\hline HPLC & High-Performance Liquid Chromatography \\
\hline HSP & Heat Shock Protein \\
\hline
\end{tabular}




\begin{tabular}{|c|c|}
\hline Hsp110 & Heat Shock Protein of $110 \mathrm{kDa}$ \\
\hline Hsp70 & Heat Shock Protein of $70 \mathrm{kDa}$ \\
\hline Hsp90 & Heat Shock Protein of $90 \mathrm{kDa}$ \\
\hline $\operatorname{Ig}$ & Immunoglobulin \\
\hline IGF1R- $\beta$ & Insulin-Like Growth Factor 1 Receptor $\beta$ Chain \\
\hline INPP4B & Inositol Polyphosphate 4-Phosphatase Type II \\
\hline INPP5K & Inositol Polyphosphate-5-Phosphatase K \\
\hline IR & Insulin Receptor \\
\hline IRE $1 \alpha$ & Inositol-Requiring Enzyme $1 \alpha$ \\
\hline IRW & Inveon Research Workspace \\
\hline LBLs & EBV-Transformed B-Cell Lymphoblastoid Cell Lines \\
\hline LC & Immunoglobulin Light Chain \\
\hline $\mathrm{LC} 3 \mathrm{~B}$ & Microtubule-Associated Protein 1 Light Chain 3 Beta \\
\hline MIGIRKO & Muscle-specific Dual IGF1R and IR Knockout Mice \\
\hline MS & Mass Spectrometry \\
\hline MSS & Marinesco-Sjögren Syndrome \\
\hline MyHC & Myosin Heavy Chain \\
\hline NBD & Nucleotide-Binding Domain \\
\hline NEFs & Nucleotide Exchange Factors \\
\hline $\mathrm{NP}_{15}-\mathrm{KLH}$ & $\begin{array}{l}\text { 4-Hydroxy-3-nitrophenylacetyl conjugated to Keyhole Limpet } \\
\text { Hemocyanin }\end{array}$ \\
\hline OSEM & Ordered Subset Expectation Maximization \\
\hline OST & Oligosaccharyltransferase \\
\hline PBA & Sodium 4-phenylbutyrate \\
\hline
\end{tabular}




$\begin{array}{ll}\text { PDGFR- } \alpha & \text { Platelet-Derived Growth Factor Receptor } \alpha \text { Chain } \\ \text { PDI } & \text { Protein Disulfide Isomerase } \\ \text { PERK } & \text { dsRNA-Activated Protein Kinase-Like Protein Kinase } \\ \text { PMSF } & \text { Phenylmethylsulphonyl Fluoride } \\ \text { qPCR } & \text { Quantitative Real Time PCR } \\ \text { RIDD } & \text { IRE1-Dependent Decay Of mRNA } \\ \text { RIPK1 } & \text { Receptor-Interacting Serine/Threonine-Protein Kinase 1 } \\ \text { ROS } & \text { Reactive Oxygen Species } \\ \text { RYR } & \text { Ryanodine Receptors } \\ \text { SBD } & \text { Substrate-Binding Domain } \\ \text { sHSP } & \text { Small Heat Shock Protein } \\ \text { SIL1 } & \text { Suppressor of Irel And Lhs1 Deletion 1 } \\ \text { SNAP2 } & \text { Screening for Non-Acceptable Polymorphisms, Version 2 } \\ \text { SRP } & \text { Signal Recognition Particle } \\ \text { sXBP1 } & \text { Uauroursodeoxycholic Acid } \\ \text { TFEB } & \text { Spliced XBP1 } \\ \text { TMD } & \text { Transcription Factor EB } \\ \text { TMTs } & \text { Transmembrane Domain } \\ \text { TUDCA } & \text { Tandem Mass Tags } \\ \text { UGGT } & \text { UPR }\end{array}$


VOIs

YY1

${ }^{18}$ F-FDG
Volumes of Interests

Ying Yang 1

18F-Labeled Fluorodeoxyglucose 


\section{CHAPTER 1. INTRODUCTION}

\section{Biosynthesis of Secretory Pathway Proteins - Journey into the ER}

Nearly a third of human genomic open reading frames encode proteins that enter the Endoplasmic Reticulum (ER) and traverse the secretory pathway to reach their final destination as residents of single membrane-bound organelles, plasma membrane, or the extracellular space (1). As the newly synthesized polypeptide chain emerges from the ribosome, the signal recognition particle (SRP) recognizes the typically N-terminal signal sequence and targets the ribosomal-polypeptide-SRP complex to the ER-localized SRP receptor (2). The ribosome-polypeptide chain complex is then directed towards a protein-conducting channel in the ER membrane called the Sec61 translocon. The Sec61 translocon allows efficient vectorial transport of the growing polypeptide across the ER membrane. Co-translational targeting of secretory pathway proteins via the SRP pathway is very well characterized, but all proteins do not exclusively adhere to this mode of ERentry. Tail-anchored proteins, one such exception to this mode of translocation, are generally cytosolically oriented and consist of a C-terminal transmembrane domain, which is occluded by the ribosome while the protein is being synthesized. Thus, the availability of the transmembrane domain for recognition and insertion into the ER membrane can only occur post-synthesis and requires an SRP-Sec61 complexindependent mechanism in this scenario. This alternative mode of insertion into the ER membrane is mediated by a group of proteins belonging to the GET (Guided Entry of TA) pathway (3).

The nascent polypeptide entering the ER undergoes a series of protein processing and modification events, which may include cleavage of the signal sequence, N-linked glycosylation, intra- and inter-molecular disulfide bond formation, and oligomerization of subunits in the case of multimeric proteins. In parallel to these events, the maturing polypeptide also begins to fold into three-dimensional secondary and tertiary structures governed by its amino acid sequence and macromolecular environment. During the process of protein folding, tracking the free energy of each conformation assumed by the polypeptide chain (high free energy) on its journey to becoming completely folded (stable conformation with minimal free energy) can be imagined as a 'funnel-shaped' landscape. The $\mathrm{X}$ and $\mathrm{Y}$ axes represent the conformational diversity that the protein can assume whereas the $\mathrm{Z}$ axis tracks the free energy of the assumed conformation. As the polypeptide begins to form intrachain hydrophobic and electrostatic associations on its journey to attain the native state conformational ensemble, it traverses down the free energy funnel with each step restricting its conformational diversity (Figure 1-1). However, this landscape is more aptly a 'rough terrain' and certain protein conformations, while lower in their free energy, are non-native and need to be reversed for the folding polypeptide to expand its conformation reach. These 'pitfalls' in the free energy landscape may kinetically trap the protein in a native intermediate state or an aggregation-prone intermediate state or an aggregated conformation, the latter of which might be even more thermodynamically stable than the native conformation itself $(4,5)$. From an evolutionary perspective, the cell has made an enormous investment to facilitate 


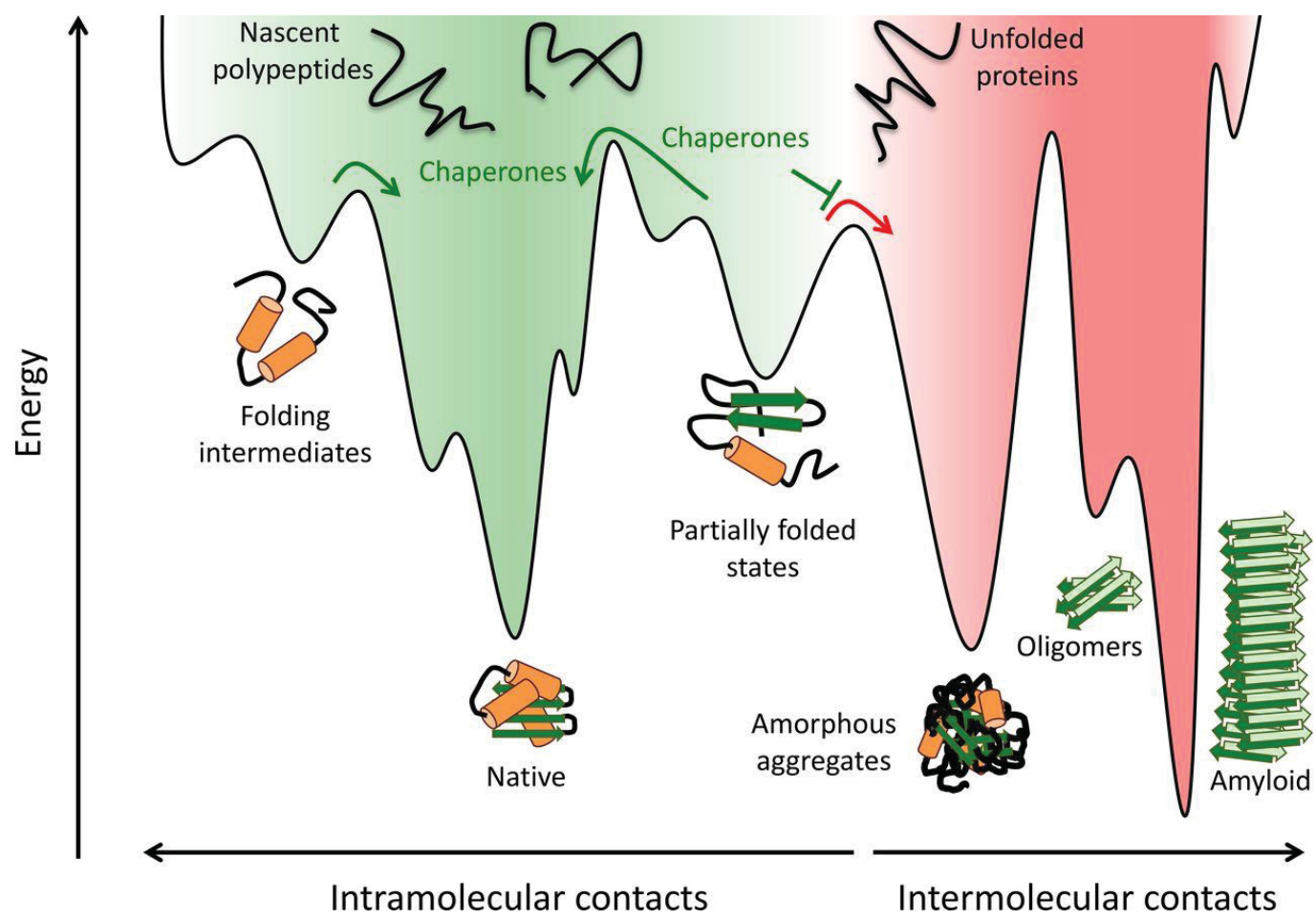

Figure 1-1. The free energy landscape during protein folding and aggregation. Protein folding and aggregation. Nascent polypeptides fold by sampling various conformations and sequestering hydrophobic amino acid residues. Partially folded intermediates, both on- and off-pathway, can become trapped in localized energy minima. These species are at risk of aggregation by forming aberrant intermolecular contacts, which can lead to the formation of oligomers, amorphous aggregates, and amyloid fibrils. Molecular chaperones promote the formation of the native species by lowering freeenergy barriers between kinetically stable intermediates, smoothing the protein folding landscape (green arrows), and preventing aberrant intermolecular interactions (red arrow).

Reprinted with permission from Rockefeller University Press. Klaips CL, Jayaraj GG, Hartl FU. Pathways of cellular proteostasis in aging and disease. J Cell Biol. 2018 Jan 2;217(1):51-63. 
proper protein folding, evolve mechanisms to deal with misfolding rapidly, and to avoid the formation of toxic protein species that may prove detrimental to its health. The stability of a protein's three-dimensional shape and the conformation required to facilitate efficient biological function present two competing evolutionary pressures. For example, a protein which might have improved biological function may not possess the right biophysical properties to fold efficiently. Conversely, a protein that may fold efficiently, might not be able to mediate sufficient function at its cellular concentration (6).

Space can be a constraint for protein folding. The ribosomal tunnel and the Sec61 translocon can only accommodate $\alpha$-helix formation within the emerging polypeptide chain (7). The formation of other secondary structures like $\beta$ sheets and turns requires greater spatial and conformational freedom, which is considerably expanded upon entry into the ER. This expansion of conformation freedom also increases the risk for unfavorable intrachain interactions. On the other hand, the ER is nearly 30 times more oxidizing with the ratio of reduced glutathione to the disulfide form (GSH/GSSG) within the secretory pathway ranging from $1: 1$ to $3: 1$, whereas the overall cellular GSH/GSSG ratio ranges from 30:1 to 100:1 (8); has a high concentration of calcium ions (close to $1 \mathrm{mM}$ ); and is devoid of any proteolytic machinery. To combat this environment, the ER is equipped with a multitude of molecular chaperones and folding factors that serve to shelter the nascent polypeptide chain entering the ER and restrict its conformational landscape. This slows down the overall folding process and prevents counterproductive folding, while governing efficient protein processing, modifications, folding, and oligomeric assembly.

\section{Protein Folding and Quality Control in the Endoplasmic Reticulum}

The endoplasmic reticulum possesses a stringent protein quality control (ERQC) system for newly synthesized secretory pathway proteins. The ER consists of two major families of molecular chaperones, the Heat Shock Protein (HSP) chaperones, and the glycan-binding lectin chaperones, both of which are complemented by their diverse machinery of co-chaperones. These chaperone families are additionally complemented by peptidyl prolyl cis/trans isomerases, which allow isomerization of the cis and trans peptide bonds within the amino acid proline, oxidoreductases, and client-specific folding factors. The resident chaperones and folding factors inhibit, delay, and modulate protein folding to ensure that folding intermediates, misfolded or aggregated proteins, and assembly-incompetent or orphan subunits of oligomeric proteins remain within the ER and do not undergo faulty transportation along the secretory pathway (9).

Despite all the cellular resources dedicated to protein folding, a significant fraction of the newly synthesized polypeptides fail to attain their correct native structure (10). These aberrantly folded proteins residing in ER need to be degraded by the cell. There are two primary means of degrading proteins, by either employing the ubiquitin proteasomal system (UPS) or by the autophagy-lysosomal machinery. The terminally misfolded and unassembled subunits of oligomers are recognized by the chaperones and co-chaperones of the ERQC machinery. These chaperones target the misfolded protein 
for retrotranslocation via a protein-conducting channel (conceptually similar to the translocon and is referred to as the retrotranslocon) to the cytosol, where it is simultaneously ubiquitinated by the ER-membrane-resident ubiquitin ligase complexes and selectively targeted for degradation by the cytosolic proteasome. This process is referred to as ER-associated degradation (ERAD) (11). It has been suggested that under rate-limiting conditions of ERAD, excessive amounts of soluble proteins are capable of traversing the secretory pathway up to the trans-Golgi network where they are selectively targeted to the lysosome (12). However, dealing with protein aggregates sequestered within the ER pose a challenge for the ERAD machinery since these aggregates cannot be efficiently extracted through the retrotranslocon. Such ERAD-incompetent aggregates are degraded by the autophagy-lysosomal system $(12,13)$. There are two components to the autophagy-mediated degradation of ER-aggregates, macroautophagy and ER-phagy. Macroautophagy is characterized by the generation of double membranous transport vesicles called autophagosomes that engulf their cargo as they are formed. These autophagosomes subsequently fuse with the lysosomal compartment upon which the autophagosomal cargo is degraded by the lysosome-resident lytic enzymes. ER-phagy, on the contrary, is an organelle-selective process which occurs independently of the core autophagy components and is characterized by the formation of ER-whorls which are subsequently taken up by the lysosome (14).

\section{Chaperone Families of the Endoplasmic Reticulum}

\section{Lectin chaperones and co-chaperones}

Amongst numerous ER-resident chaperones, the local HSP chaperone superfamily includes BiP/Grp78 (Hsp70), Grp94 (Hsp90), and Grp170 (Hsp110). While these chaperones serve as the ER luminal counterparts of cytosolic orthologs, lectin chaperones are exclusively expressed in the ER and include Calnexin (CNX) and Calreticulin (CRT) (Figure 1-2).

Calnexin is a type I membrane protein and is tethered to the ER membrane whereas Calreticulin is its soluble paralog. The lectin chaperones share 39\% sequence identity and structural similarities (15). Even though both of these chaperones have similar lectin binding preferences, they have distinct specificities for the location of the glycans they bind and also the number of glycans they recognize. Thus, it is postulated that Calnexin and Calreticulin recognize distinct topologies. Calnexin and Calreticulin are also capable of binding to polypeptide regions of both glycosylated and nonglycosylated substrates, albeit with altered kinetic properties, and can even suppress their aggregation (16-18). Calnexin and Calreticulin play crucial roles in ER protein quality control and calcium homeostasis and are involved in several processes in immunity, cancer, and apoptosis. These lectin chaperones are further aided by their set of co-chaperones and folding factors. The oligosaccharyltransferase (OST) complex is

responsible for covalently transferring an oligosaccharide sugar moiety from a lipidbound dolichol donor to an asparagine residue within an N-X-S/T motif (where X cannot 


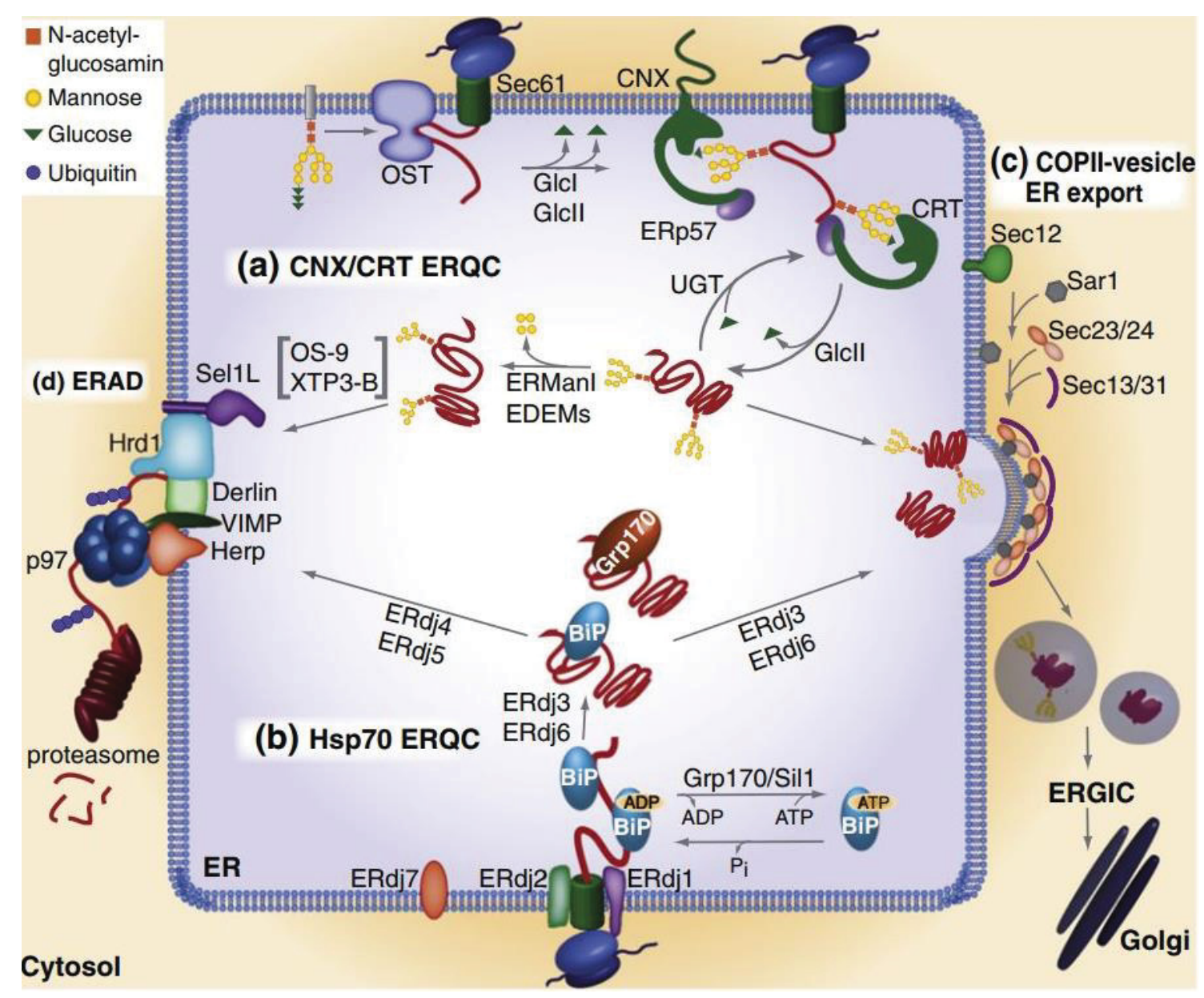

Figure 1-2. The ER protein quality control machinery.

The schematic highlights the role of various co-chaperones and folding factors that assis the two main chaperone systems within the ER. The lectins calnexin/calreticulin (CNX/CRT) (a) and the Hsp70 chaperone BiP (b), aid in the proper folding of secretory pathway proteins (c) or recognize and target them for ER-associated degradation by the cytosolic $26 \mathrm{~S}$ proteasome if folding ultimately fails (d).

Reproduced with permission from Elsevier. Behnke J, Feige MJ, Hendershot LM. BiP and its nucleotide exchange factors Grp170 and Sil1: mechanisms of action and biological functions. Journal of molecular biology. 2015;427(7):1589-608. Epub 2015/02/24. doi: 10.1016/j.jmb.2015.02.011. PubMed PMID: 25698114; PubMed Central PMCID: PMCPMC4356644. 
be a proline residue) flanked by a flexible region in the polypeptide that is entering the ER. This large hydrophilic oligosaccharide itself can affect the solubility and folding properties of the protein. It can be acted upon by multiple ER-resident glycosyl-enzymes that modify this sugar moiety, resulting in a ligand that is recognized by the ER-resident carbohydrate-binding proteins. Glycosidases I and II (GlcI and GlcII, respectively) trim the glucose oligosaccharide residues, which allows the unfolded, monoglucosylated glycoprotein to be recognized by either Calnexin or Calreticulin. These chaperones act in concert with ERp57, the lectin-associated oxidoreductase, which catalyzes the formation of native disulfide bonds in the folding polypeptides. Some proteins can fold correctly after a single interaction with the CNX/CRT chaperones. However, numerous proteins require more than one cycle of association. Incompletely folded intermediates containing non-native structures can be sensed by the bifunctional UDP-glucose glycoprotein glucosyltransferase (UGGT) sensor, which allows it to 'inspect' non-native structures within folding polypeptides and re-glycosylate the previously deglycosylated substrates, allowing them to re-engage with CNX/CRT. Glycoproteins are offered a temporal window to complete their folding. When the native structure is achieved, glycoproteins are exported from the ER in cytosolic coatamer protein II (COPII)-coated vesicles. If folding ultimately fails, proteins are further targeted for ERAD by an ER mannosidase or an ER degradation enhancing $\alpha$-mannosidase-like protein (EDEM), resulting in removal of 4 mannose residues, and OS-9 and XTP3-B, which allow recognition of misfolded proteins and ERAD-targeting (19).

\section{The HSP70 chaperone BiP/Grp78: It's ATPase cycle and its co-chaperone network}

Pre-B lymphocytes and hybridomas derived from these lymphocytes synthesize the immunoglobulin (Ig) heavy chain alone, without the light chain. These Ig heavy chains were identified to non-covalently associate with a $78 \mathrm{kDa}$ protein named immunoglobulin heavy chain binding protein-BiP (20). Soon after, it was determined that $\mathrm{BiP}$ was an ER-resident protein that transiently, but specifically, bound to free and incompletely assembled intracellular heavy chains with an unfolded Ig domain, and dissociated when the Ig heavy chain was completely folded when it associated with the Ig light chain. The association of BiP with Ig heavy chains retained them within the ER, thereby implicating $\mathrm{BiP}$ as a necessary regulator to prevent premature escape and secretion of incompletely assembled Ig molecules in the Ig biosynthetic process $(21,22)$. Moreover, it has also been shown that BiP plays a key role in governing the half-life of a protein and was capable of delivering partially folded substrates for degradation by the proteasome (23). However, the mere presence of a BiP-binding sequence within a client protein is not an absolute indication of BiP's binding ability. This interaction is rather governed by the kinetic rate of folding and stability of the domain-containing protein (24). This work provided one of the earliest evidence for the crucial role of BiP in ER protein quality control and the biosynthesis of immunoglobulins $(21,22,25)$. It was later demonstrated that BiP and Grp78 were the same protein and that BiP was capable of binding other proteins as well, such as the coagulation Factor VIII $(25,26)$. In subsequent studies, it has been demonstrated that BiP can be posttranslationally modified by AMPylation or phosphorylation and this modification is characteristic of $\mathrm{BiP}$ that was 
that was not bound to its substrates. This indicates that are at least three forms of the cellular BiP pool: BiP complexed with its substrates, a free and unmodified monomer, and free modified oligomers, implying that the modified oligomeric BiP species serve as a cellular reservoir, which can be readily mobilized to accommodate fluctuations in the secretory proteome (27-32). BiP also plays a crucial role in the disposal of nonglycosylated substrates and this process relies on the retrotranslocation complex, including the ER transmembrane proteins Derlin-1, Herp, Hrd1, Sel1 L, and VIMP. These proteins connect the ER luminal chaperone machinery to the to the 26S proteasomal degradation machinery via $\mathrm{VCP} / \mathrm{p} 97$, which provides the energy required for extraction of a protein through the retrotranslocon channel (33).

Work from Dr. Amy S. Lee's laboratory has shown that the glucose-regulated proteins can also be upregulated by a variety of cellular stresses in addition to glucose depletion and play a cytoprotective role. These stresses include the increased expression of misfolded and underglycosylated proteins, oxidative stress, heat shock, and disruption of ER calcium homeostasis (34). It has been shown that BiP is upregulated in the inner cell mass and the trophectoderm during embryonic development stage 3.5, and biallelic deletion of BiP leads to lethality due to a peri-implantation defect. Thus, BiP is crucial for embryonic cell growth and survival of pluripotent stem cells (35). Interestingly, haploinsufficiency of BiP in mice does not affect viability. These mice upregulate Grp94 and protein disulfide isomerase (PDI), which facilitates the correct arrangement of disulfide bonds within a protein (35). In support of the crucial importance of BiP in cellular systems, a highly virulent Escherichia coli strain that was responsible for the hemolytic uraemic syndrome endemic in Australia in 1998 was found to synthesize a cytotoxin that mediated its toxicity by inactivating BiP, cleaving its interdomain linker, which is necessary for the allosteric relay between BiP's NBD and SBD (36).

Mammalian Grp promoters, including that of Grp78, contain multiple copies of ER stress response elements (ERSE) and UPR response elements (UPRE), which enable the glucose-regulated chaperones to be upregulated upon insults to the ER which compromise its homeostasis. The ERSE, an evolutionarily conserved tripartite sequence, typically consists of a CCAAT $\left(\mathrm{N}_{9}\right) \mathrm{CCACG}$ (N represents a 9-bp GC-rich region) consensus sequence and is more complex than the UPRE. The transcription factors, NFY and Ying Yang 1 (YY1), both have been reported to enhance the Grp78 promoter activity under conditions of ER stress. While NF-Y binds to the CCAAT motif of the ERSE, YY1 binds to the CCACG sequence. In addition, the UPR-sensor ATF6 has also been reported to bind to the Grp promoters in complex with NF-Y (34).

Hsp70s are highly conserved from $E$. coli to mammals with nearly $40 \%$ sequence identity (37). In addition to BiP's role in de novo protein folding and assembly, BiP plays a key role in regulating ER homeostasis. BiP maintains the permeability barrier of the ER membrane by sealing the luminal face of the translocon channels that are either not in an active state of conducting protein or newly targeted translocons that are associated with a ribosome but haven't yet begun translational translocation (38). BiP is also capable of recognizing and stably associating with unfolded regions within a client protein and targeting them to the cellular degradation machineries (23). BiP can also 
reversibly associate with the luminal domains of UPR transducers and maintain them in a dormant state (39). In addition, $\mathrm{BiP}$ has been shown to be a bona fide ER calcium storage protein, enhancing the ER's storage capacity by approximately $25 \%$ under resting conditions (40). Except for BiP's contribution to the ER's calcium stores, all other functions rely on the ability of BiP to bind adenosine triphosphate (ATP) and succesfully hydrolyze it. Evidence from our laboratory has indicated that mutation of the residues on BiP's nucleotide binding domain that interact with ATP or ADP or of residues results in severe inhibition of its ATPase activity. The mutant BiP is still able to bind ATP and Ig heavy chains, however, it is deficient in the release of the bound Ig heavy chains (41). Intriguingly, the expression of these BiP mutants in COS cells not only recapitulated the defect in BiP being able to efficiently release client proteins, but also revealed the importance of BiP in maintaining the integrity of ER and preventing disruption of ER morphology and dilation (42).

BiP's ATPase activity is tightly regulated by two families of co-chaperones, ERresident DnaJ-like proteins (ERdjs) and nucleotide exchange factors (NEFs) (Figure 1-3). Similar to other members of the Hsp70 family, BiP has an N-terminal nucleotidebinding domain (NBD) and a C-terminal substrate-binding domain (SBD), which are connected by an interdomain linker. BiP can exist as two distinct nucleotide-bound conformations, which dictate the conformation adopted by the SBD through ligandinduced conformational changes. Recent evidence suggests that the ATP-bound state of $\mathrm{BiP}$ is a completely closed conformation, which does not allow BiP to engage any polypeptides via its SBD (43). BiP's inherent ATPase activity is weak. The association of BiP with the ER-resident Hsp40 co-chaperones, ERdjs, via the J-domain has been postulated to facilitate a partial opening of the SBD to initiate stable binding of the substrate. This conformation is capable of rapidly engaging new susbtrates. However, the affinity of this comformation for clients is low and they are rapidly released as well. Association of BiP with ERdjs and the substrate together stimulates BiP's ATPase activity, leading to the hyrolysis of the bound ATP molecule to ADP and subsequent dissociation of the ERdj protein. Multiple ERDJs (ERDJ3-6) have also been reported to engage substrates themselves directly and transfer them to BiP upon association. The ADP-bound BiP conformation possesses high-affinity for the substrate and serves as a holdase for its substrate, preventing off-target folding pathways. However, the substrate cannot fold while bound to BiP since the SBD's closed lid restricts the substrate's conformational freedom and need to be released from BiP on its own and require the function of NEFs. There are two ER-resident co-chaperones, which have been reported to possess NEF-activity towards BiP, Sill and Grp170. Interestingly, Grp170 has been recently demonstrated to also function as a bona fide ER chaperone that is capable of binding incompletely folded substrates and is regulated by a mechanism distinct from that of BiP (44). NEFs introduce a torsional strain within the BiP-NBD, which allows disengagement of the bound ADP molecule by breaking the hydrogen bonded contacts. The release of the ADP molecule allows the BiP-NBD to engage a new ATP molecule, which causes an active release of the bound polypeptide substrate and closes

the SBD. This mechanism prevents BiP from repeatedly engaging the same substrate and additionally provides a spatial and temporal window in which that substrate may fold (43, 45). 


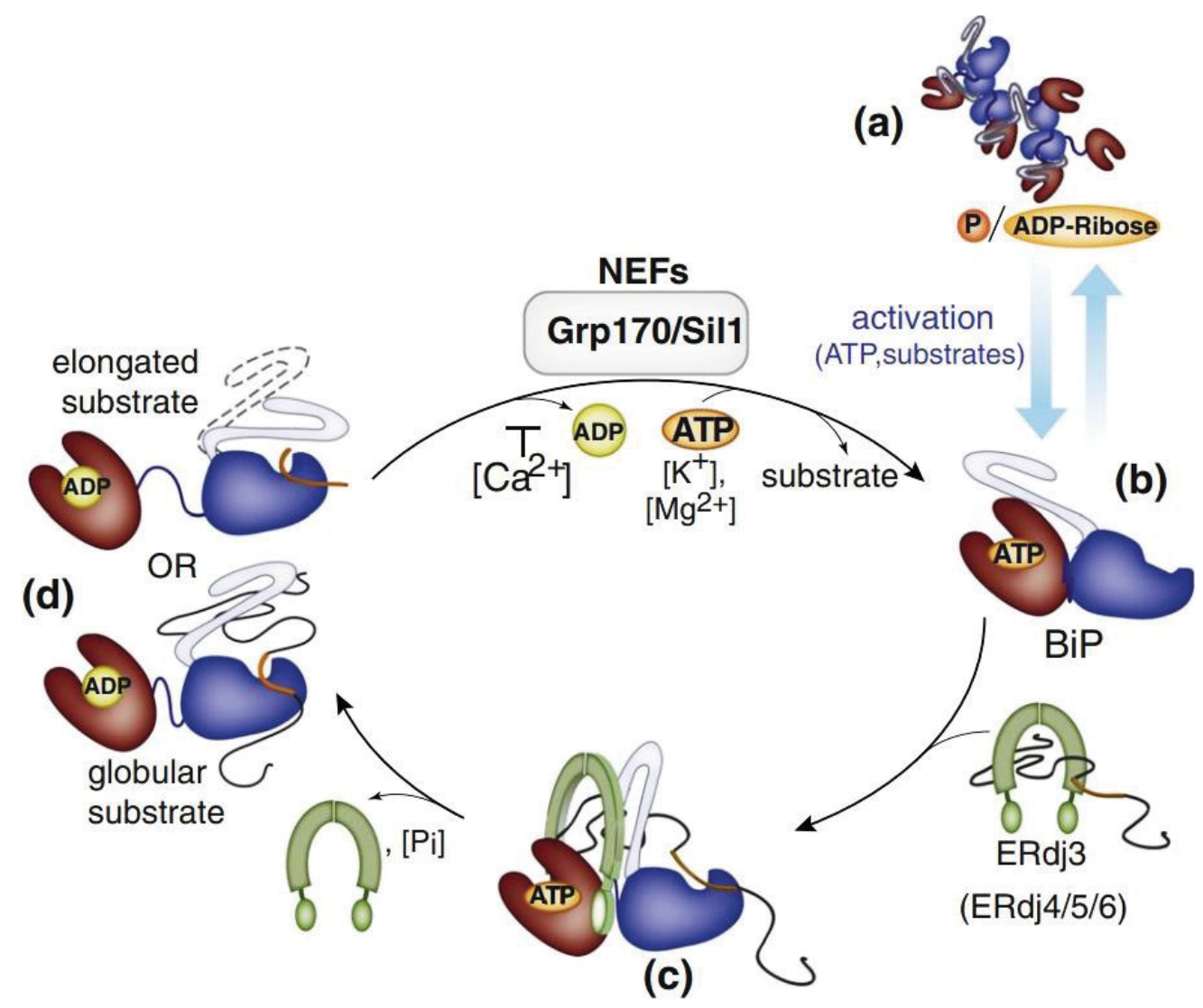

Figure 1-3. The ATPase cycle of $\mathrm{BiP}$ and the co-chaperones regulating it.

Reproduced with permission from Elsevier. Behnke J, Feige MJ, Hendershot LM. BiP and its nucleotide exchange factors Grp170 and Sil1: mechanisms of action and biological functions. Journal of molecular biology. 2015;427(7):1589-608. Epub 2015/02/24. doi: 10.1016/j.jmb.2015.02.011. PubMed PMID: 25698114; PubMed Central PMCID: PMCPMC4356644. 


\section{Grp170}

Lhs1p's (the yeast homolog of Grp170) discovery stemmed from the yeast genome sequencing project, which revealed an open reading frame that would encode a novel Hsp70-like protein. Indeed, this protein was shown to be an ER-luminal glycoprotein that is induced upon UPR-activation and was designated as luminal Hsp70 or Lhs1. Further efforts to characterize the role of this protein revealed that the deletion of Lhs $1 p$ does not yield a lethal phenotype, unlike the deletion of $\operatorname{Kar} 2 p$ (the yeast homolog of BiP). However, Lhs $1 p$ deletion resulted in an activation of the UPR and a severe defect in the translocation of multiple secretory pathway proteins. The combined deletion of Lhs Ip with Irelp, which alone would blunt the cell's ability to activate the ER stress response, or along with $\operatorname{Kar} 2 p$, both resulted in cellular lethality. Thus, Lhs1p was postulated to be an ER luminal chaperone important for polypeptide translocation into the ER and subsequent protein folding of secretory pathway proteins, and was shown to contain an UPRE in its promoter region, similar to that of $\operatorname{Kar} 2 p$, which enabled induction of this genetic locus upon UPR activation (46, 47). Surprisingly, studies in yeast and mammals later demonstrated that the postulated molecular chaperone Lhs $1 \mathrm{p}$ and its human homolog, Grp170, are also capable of nucleotide exchange activity for their respective Hsp70-partners, Kar2p and BiP. It was also shown that Kar2p reciprocally activated the ATPase activity of Lhs1p, indicating that the ATPase activity of Lhs1p and Kar2p were coupled, which was important for normal ER function in vivo (48-50). Lhs $1 p$ has also been shown to play an important role in the conformational repair, refolding, and stabilization of denatured protein aggregates, similar to its cytosolic ortholog Hsp104 (51, 52). Dr. Bernd Bukau's group further demonstrated that the mechanism of Grp170's nucleotide exchange was similar to that of the cytosolic Hsp110 (53). Grp170 is conserved in eukaryotes and is ubiquitously expressed within humans. It contains an N-terminal ER-targeting signal sequence, NBD, followed by a $\beta$-sheet and an $\alpha$-helical domain and a C-terminal KNDEL ER-retention sequence. The nucleotide binding domain of Grp170 is very similar to that of BiP and both of these proteins overall share a similar domain organization with each other and with conventional Hsp70s (33). It has been shown that Grp170 and BiP are capable of binding to the same molecular species of different substrates and that the dissociation of BiP from the substrate was not a prerequisite for Grp170-binding (44). However, there are functional and structural differences between the Grp170 and conventional Hsp70s. The binding of Hsp70substrate complex is governed by the occupancy of the nucleotide binding domain. When ATP occupies the NBD, the substrate is released from Hsp70. However, consistent with the disaggregase activity shown for Hsp110, the occupancy of Grp170's NBD by either ATP or ADP does not influence substrate release from this molecular chaperone. In addition, using mutational analysis, the $\alpha$-helical domain of Grp170 and the unstructured loop docked within its $\beta$-sheet domain have also been reported to influence Grp170's substrate-binding ability (44). Recent evidence has highlighted that Grp170 can facilitate the degradation of certain substrates, as observed in the case of the epithelial sodium channel subunit (54). 


\section{ERdjs}

ERdjs are characterized by the presence of a J-domain, which mediates association of Hsp40 members with their respective Hsp70-binding partners. There are eight ERdjs (Erdj1-8) reported to date $(55,56)$. ERdjs primarily serve two functions. They enable BiP to engage its substrate by transforming the ATP-bound BiP to an open conformation (43) and, secondly, deliver the substrate to BiP. ERdjs can be categorized into three groups on the basis of their structural characteristics. Type I ERdjs (Erdj3) consist of an N-terminal J domain, a glycine/phenylalanine-rich domain, and a substratebinding domain, which contains a cysteine-rich zinc-binding domain. Type II proteins (ERdj4) possess only the J-domain and the glycine/phenylalanine-rich domain and poorly-defined substrate-binding site. Type III ERdjs only have a J domain (ERdjs1/2/5/6). ERdjs are present at substoichiometric amounts compared to BiP and have been shown to vary widely in their concentrations among each other within a cell. These DnaJ-like proteins also vary in their ability to be induced by the UPR and only ERdjs3/4/5/6 have been reported to show this induction. Growing evidence suggests that ERdjs play a crucial role in determining the fate of a substrate (55).

ERdj1, a transmembrane protein, serves as a translational checkpoint by dually associating with the translating ribosome via its cytosolic domain and with BiP via its $\mathrm{J}$ domain. ERdj1 ensures that BiP is present in the vicinity to engage newly synthesized polypeptides as they enter the ER lumen. ERdj2 is a multipass transmembrane protein, has been shown to associate with the translocon channel, and plays an important role in translocation of proteins. ERdj3 has been independently identified as an Hsp40 cochaperone that assists in the trafficking of Shiga toxin and also as a part of the chaperone complex that binds unassembled Ig heavy chains. ERdj3 plays a major role in protein folding and even in ERAD of certain substrates by maintaining them in a soluble state compatible with ERAD. ERdj4 is the least abundant ERdj, which also displays the greatest induction upon activation of the UPR. ERdj4 has been shown to play a role in the ERAD of certain clients of BiP. ERdj5 possesses four thioredoxin domains in addition to the $\mathrm{J}$ domain and has been shown to interact with the retrotranslocon machinery. ERdj5 is an oxidoreductase and has been shown to reduce the disulfide linkage in ERAD-targeted substrates, allowing efficient retrotranslocation. Recently, it has also been suggested that ERdj5 is involved in maintaining ER calcium homeostasis via ERdj5-mediated regulation of the ER calcium channel, SERCA2b. ERdj6 has been shown to possess three tetratricopeptide domains, which allows it to engage unfolded proteins, and is postulated to play a pro-folding role in the ER lumen. ERdj7 is a transmembrane protein with an ER-luminal J domain. However, little is know about is known about its function within the cell. ERdj8 is the most recently discovered ERdj protein and is suggested to be prevalent at ER-mitochondrial association sites where it play a role in negatively regulating autophagy $(55,56)$.

The ATPase cycle of BiP is crucial to its function as a substrate holdase. Mutations in BiP that severely inhibit its ATPase activity prevent the release of substrates from $\mathrm{BiP}$, as exemplified in the case of Ig heavy chains and light chains, and even prevents their secretion $(41,57)$. The binding of $\mathrm{BiP}$ to its substrates is rather 
promiscuous, which allows it to bind a diverse set of substrates. It is known that BiP preferentially binds a 7-9 amino acid sequence that is composed of alternating aromatic and hydrophobic amino acid residues. This sequence is generally buried within the native conformation of a relevant protein but is exposed when it is unfolded or misfolded (58). A recent study from our laboratory confirmed and expanded upon previous findings on BiP-binding sequences, using Ig as a model system to reveal intriguing findings into the nature of substrate engagement by BiP, Grp170, and ERdjs3-5. This study found that $\mathrm{BiP}$ and Erdj3 have numerous binding sites within an Ig, which likely allows efficient recognition upon entry into the ER. Contrarily, Grp10, Erdj4, and Erdj5, all recognized nearly identical sequences, which display a high propensity for aggregate formation and have been implicated to play an important role in ERAD. This suggests a convergence in evolutionary mechanisms to stringently deal with aggregates (59).

\section{SIL1}

The first nucleotide exchange factor was not identified until the mid-1990s when several laboratories independently discovered them in three organisms. A conditional lethality screen in the yeast Yarrowia lipolytica to detect cellular components which interacted with the 7S RNA or SRP in the process of co-translational translocation led to the identification of the Sls $1 p$ gene (60). The Sls1p-encoded protein was an ER-luminal, 426-amino acid-long polypeptide, which contained an N-terminal signal sequence and a C-terminal ER-retention motif. Sls1p fractionated with the membranous fraction and was shown to interact with the Sec61p translocation apparatus. It was also determined that this gene was induced by ER stresses such as heat shock and inhibition of glycosylation. Deletion of the $S l s 1 p$ gene resulted in a temperature-sensitive growth phenotype and resulted in decreased levels of mature secretory proteins. Interestingly, overexpression of Sls1p had a similar phenotype, suggesting a cellular stoichiometric preference for the amount of Sls1p. This protein was also shown to bind Kar2p (the yeast homolog of BiP) and disruption of this interaction significantly affected the secretion of a cotranslationally translocated reporter protein (61). Thus, Sls1p was thought to act in the translocation process where it assisted in the ER-luminal entry of secretory pathway proteins or their folding (60).

Sill was identified as a gene whose overexpression rescued the severe growth defect observed in yeast $\Delta$ Irel 1 Lhs 1 double mutants and, thus, was named as the suppressor of Irel and $\underline{L} h s 1$ deletion $\underline{1}$ (Sil1). Sillp was determined to an ER-resident protein, which bound the ATPase domain of Kar2p (62). It was further shown that although deletion of Sillp on its own was not lethal, a combined deletion of Sillp and Lhs 1 proved to be lethal with an absolute block in protein translocation into the ER (62).

Mammalian (human) Sil1, was discovered in our laboratory using a yeast twohybrid screen aimed at identifying proteins which interacted with a mutant ATPase domain of BiP and was initially named as the BiP-associated protein (BAP) (63). The BAP gene encoded a 461-amino acid protein with a putative N-terminal ER-targeting sequence and two N-linked glycosylation sites and displayed nucleotide exchange 
activity (63). BAP was determined to be similar to the yeast Sls 1p protein and Sillp, which interacts with the NBD of the yeast BiP homolog, and also similar to the cytosolic Hsp70-binding protein, HspBP1. It was further shown that BAP/Sil1 is ubiquitously expressed in mice and humans, and its expression displayed a tissue-specific pattern in humans that was highly similar to that of BiP, ERdj3, and ERdj4, suggesting that these co-chaperones of BiP function together $(63,64)$.

Sil1 has an elongated, "kidney bean"-like, molecular shape which consists of 16 $\alpha$-helices (A1-A16) and lacks $\beta$-sheets (Figure 1-4). The central helices A3-A14 form the Armadillo-like repeats (ARM1-ARM4) (45), which are named after the armadillo repeats found in $\beta$-catenin. Each ARM repeat is composed of three $\alpha$-helices that pack into a superhelix. From the resolved Sill structure complexed with BiP, it appears that Sill acts as a "wrench," which wraps around lobe IIb of BiP's NBD and makes additional contact with lobe Ib. Sill's interaction with the ADP-bound BiP causes lobes Ib and IIb to move away from each other by $\sim 3.7^{\circ}$, which is likely to disrupt the hydrogen bonds between ADP and BiP, leading to the release of ADP (45). Although the ER has two NEFs that mediate nucleotide exchange for BiP, it has been reported that these interactions are mechanistically distinct (Figure 1-5). The binding of Lhs1 to BiP requires the NBD as well as the linker. However, lobe IIb of the NBD is sufficient for Sillp association and point mutations in the Sillp-interacting site of BiP's NBD specifically disrupted Sillp binding, but not Kar2ps's interaction with Lhs1 (65).

Intriguing data from Dr. Carolyn Sevier's group has recently shown that Sil1p also acts as a reductant for BiP (66). Protein folding within the highly oxidizing conditions within the ER is a significant source of cellular reactive oxygen species (ROS), comparable to that of ROS generated during mitochondrial respiration. Oxidative stress has a detrimental impact on BiP's function by modifying a conserved cysteine within BiP's NBD that decouples the relay of information about NBD's nucleotide occupancy to its SBD, and thereby disrupts its ATPase cycle $(67,68)$. How this cysteine was reduced to restore BiP's normal function is not understood. Recently, it has been demonstrated that Sill has the potential to reduce the oxidized cysteine within BiP's NBD and restore BiP's function. Thus, in addition to the nucleotide exchange activity of Sil1, the Sil1-BiP pair are also important in sensing redox imbalance within the ER.

\section{The Unfolded Protein Response}

The protein folding capacity of the ER is governed by the composite capacity of the ER-resident chaperones to effectively accommodate the protein folding and processing load of newly synthesized proteins and the ability to efficiently deal with any abnormalities in the folding pathway. Cellular insults, either physiological or pharmacological, disrupt ER homeostasis and culminate in the accumulation of unfolded or misfolded proteins. This presents an additional burden on the ERQC machinery, leading to ER stress (ERS). The triage response to salvage ER stress is called as the Unfolded Protein Response (UPR), which leads to the initiation of an ER-to-nuclear signal transduction pathway to globally halt further protein synthesis and simultaneously 

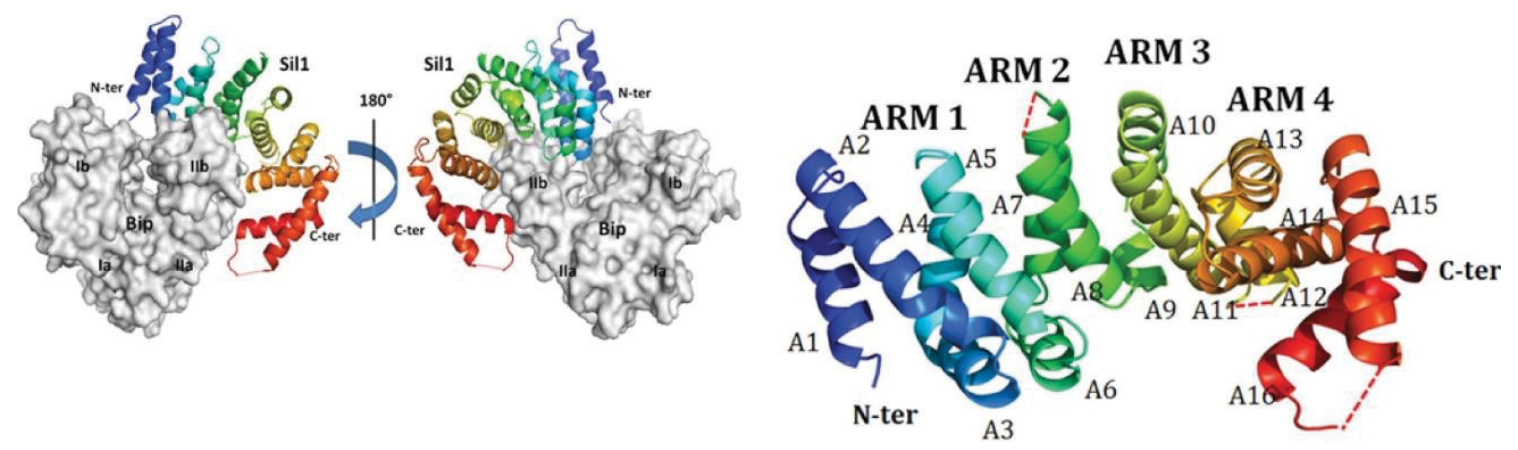

Figure 1-4. Structure of Sil1 and Sil1 complexed with BiP's NBD.

Reproduced with permission from Portland Press. Yan M, Li J, Sha B. Structural analysis of the Sill-Bip complex reveals the mechanism for Sill to function as a nucleotide-exchange factor. The Biochemical journal. 2011;438(3):447-55. Epub 2011/06/17. doi: 10.1042/bj20110500. PubMed PMID: 21675960. C Biochemical Journal. 


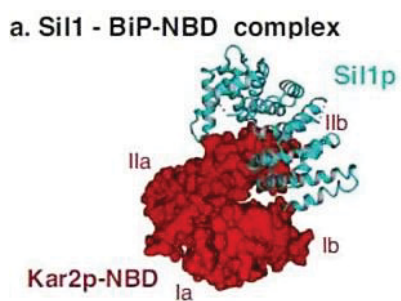

c. Grp170 - Hsp70-NBD complex

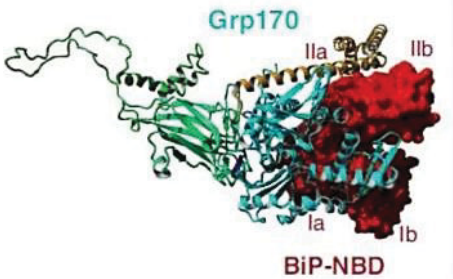

b. Sil1 - Nucleotide Exchange Mechanism
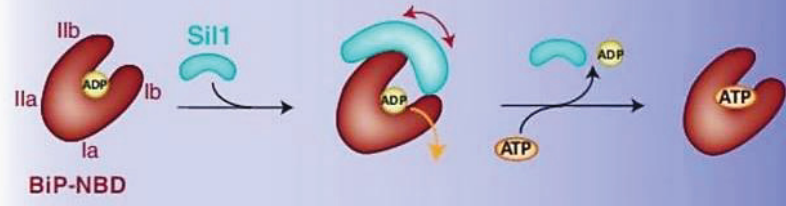

d. Grp170 - Nucleotide Exchange Mechanism

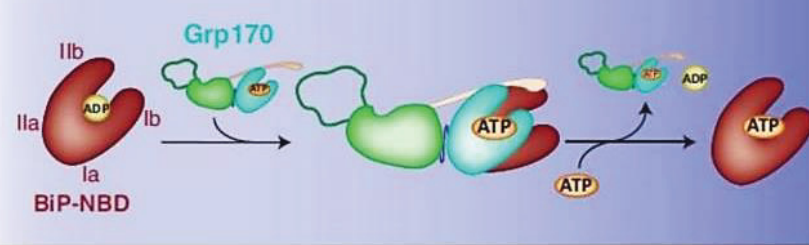

Figure 1-5. Differences in the mechanisms of nucleotide exchange by Sil1 and Grp170, the two ER-resident NEFs of BiP.

Reproduced with permission from Elsevier. Behnke J, Feige MJ, Hendershot LM. BiP and its nucleotide exchange factors Grp170 and Sil1: mechanisms of action and biological functions. Journal of molecular biology. 2015;427(7):1589-608. Epub 2015/02/24. doi: 10.1016/j.jmb.2015.02.011. PubMed PMID: 25698114; PubMed Central PMCID: PMCPMC4356644. 
enhance the ER's protein folding and degradation capacities (Figure 1-6). The UPR is orchestrated via three transmembrane proteins: dsRNA-activated protein kinase-like protein kinase (PERK), inositol-requiring enzyme $1 \alpha$ (IRE1 $\alpha)$, and activating transcription factor 6 (ATF6) (69). These UPR sensors are maintained in an inactive state by formation of a stable complex between their ER luminal domains and BiP. Perturbation of protein folding and the accumulation of unfolded and misfolded proteins in the ER sequesters BiP away from these UPR sensors, leading to their activation (39).

Dissociation of BiP from the ER-luminal binding pocket of the type I transmembrane kinase, PERK, leads to its homodimerization and autophosphorylation. This step in turn activates PERK's kinase domain that can now phosphorylate the eukaryotic initiation factor $2 \alpha$ (eIF2 $\alpha$ ) subunit, leading to a global translational initiation arrest. During this period of ER stress, a subset of mRNAs containing an internal ribosomal entry site, such as ATF4, are selectively translated. ATF4 is a transcription factor that activates the growth arrest and DNA-damage-inducible protein 34 (GADD34) and $\mathrm{C} / \mathrm{EBP}$ homologous protein (CHOP). GADD34 directs eIF2 $\alpha$ dephosphorylation to restore global mRNA translation when ER stress is resolved. On the other hand, CHOP regulates ER stress-mediated apoptosis. The expression of CHOP is initially suppressed. However, constitutive PERK activation due to unresolved or chronic ER stress overrides the adaptive phase of the UPR and leads to apoptosis (69). It has been reported that initial activation of the UPR leads to a decline in Cyclin D and Cyclin E-dependent kinase activities due to their translational inhibition, leading to a G1 cell cycle arrest. This represents an ER stress-governed cellular checkpoint that prevents cell cycle progressions in cases of disruption of cellular homeostasis (70).

Similar to PERK, IRE1 is a type I transmembrane protein with a cytosol-facing serine/threonine kinase domain. Upon dissociation of BiP from its luminal domain, IRE1 oligomerizes, leading to its autophosphorylation and subsequent activation (69). It has been reported that unfolded peptides are capable of directly binding to and activating IRE1, representing an alternative mode of IRE1 activation (71). Unlike PERK, IRE1 also has a cytosolic endoribonuclease domain, which can recognize a consensus mRNA sequence and cleaving it in a process called IRE1-dependent decay of mRNA (RIDD). Activated IRE1 also cleaves XBP1 mRNA to facilitate an unconventional splicing mechanism, which leads to a frame-shift, now encoding a transcriptionally active XBP1 protein $(\mathrm{sXBP} 1 \mathrm{~s})$. sXBP1 regulates the expression of genes whose products enhance the protein folding capacity of the ER. These genes include chaperones, co-chaperones, and components of the ERAD machinery. In addition, ectopic overexpression of sXBP1 can inhibit CHOP expression, which serves to promote cell survival (69), and can lead to a physical expansion of the ER (72). Whereas IRE1-mediated splicing of XBP1 mRNA serves a cytoprotective function, RIDD activity may preserve ER homeostasis or, upon chronic IRE1-activation, is capable of inducing cell death by apoptosis (69).

ATF6, on the contrary, is a type II transmembrane protein, consisting of a cytosolic cAMP-responsive element-binding (CREB) or ATF basic leucine zipper (bZIP) domain. ATF6 is retained within the ER by its association with BiP. When BiP dissociates from ATF6, it traffics to the Golgi, where it undergoes proteolytic processing 


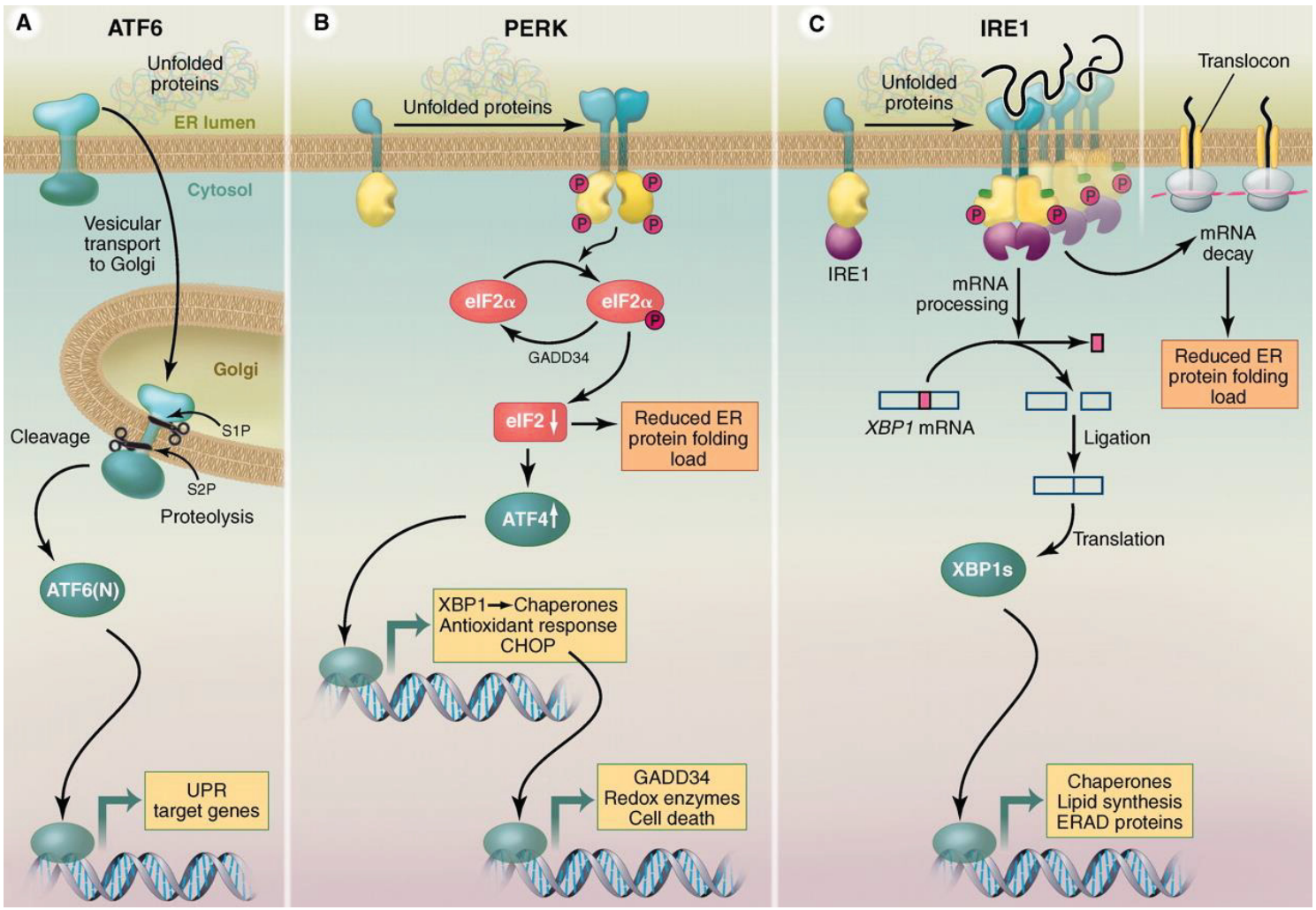

Figure 1-6. The three branches of the UPR.

(A-C) Homeostasis and stress within the ER are sensed by three families of ER transmembrane proteins that relay an ER-to-nuclear signal transduction (ATF6, PERK, and IRE1) to drive transcription of UPR target genes.

Reproduced with permission from AAAS. Walter P, Ron D. The Unfolded Protein Response: From Stress Pathway to Homeostatic Regulation. Science.

2011;334(6059):1081-6. doi: 10.1126/science.1209038 (73). 
by Golgi-resident S1P and S2P proteases to generate a transcriptionally active cleaved ATF6 protein. Cleaved ATF6 mediates the adaptive response of UPR by inducing genes that serve to increase the protein folding capacity of the ER. PERK-eIF $2 \alpha$ signaling facilitates the selective translation of ATF6, and cleaved ATF6 upregulates XBP1 mRNA, both of which serve as feed-forward loops of the UPR (69). Contrary to the association characteristics of BiP with either PERK or IRE1, the association of BiP with ATF6 is rather unique. It has been argued that the association of BiP with ATF6 cannot support a competitive model where misfolded proteins compete with ATF6 to engage BiP but is instead a relatively stable association that requires actively restarting BiP's ATPase cycle to dissociate it from ATF6 (74).

\section{Marinesco-Sjögren Syndrome}

A syndrome characterized by cerebellar ataxia, congenital cataracts, and physical and mental retardation was first described in 1931 by Marinesco and colleagues (75). Nearly 20 years later, Sjögren expanded upon the patient population with a similar phenotype, additionally reporting a hereditary nature of this disease, which was transmitted in an autosomal recessive manner (76). Thereafter, multiple additional reports followed, reporting the occurrence of this disease in an equivalent proportion of males and females and in patients of numerous nationalities and ethnicities. This whole gamut of symptoms came to be termed as the Marinesco-Sjögren Syndrome (MSS; OMIM 248800).

MSS is a multisystem disorder. However, the cardinal features used for a clinical diagnosis include cerebellar dysfunction and ataxia, bilateral cataracts, mental deterioration, impaired physical development and skeletal deformities, and progressive myopathy (77). Figure 1-7 displays the relative frequency of signs and symptoms observed in MSS (78). Cerebellar symptoms represent the most frequent and one of the earliest clinical presentations of MSS and are characterized by cerebellar ataxia due to cerebellar degeneration, primarily consisting of Purkinje and granule cell loss (64). Bilateral cataracts are nearly as frequent as the prevalence of cerebellar ataxia and precipitate during infancy with an associated visual impairment (Figure 1-7) (77). Numerous reports have noted varying characteristics of the observed bilateral cataracts, spanning from fine dot-like depositions to flaky deposits in concentric layers of the cortex and powdery opacities within the nucleus (77). However, the overall etiology has remained elusive. Mental retardation in MSS presents as learning disabilities and regressive tendencies. It is characterized by chronic atrophy of the nerve cells rather than an inflammatory process, as first reported by Marinesco $(75,77)$. The atrophy of skeletal muscles and accompanying hypotonia are also one of the most frequent symptoms of MSS, and the earliest reports indicate a progressive nature of these symptoms. Myopathy has been emphasized to be a cardinal feature and an early sign of MSS (79). It is characterized by variations in fiber size, rounding and hypertrophy, degeneration and regeneration of fibers, internalization of myonuclei, infiltration of endomysial fat, and fibrosis (79). Sewry, Voit, and Dubowitz revealed the presence of membranous autophagic whorls, particularly, a membranous structure surrounding the nucleus (80). A 


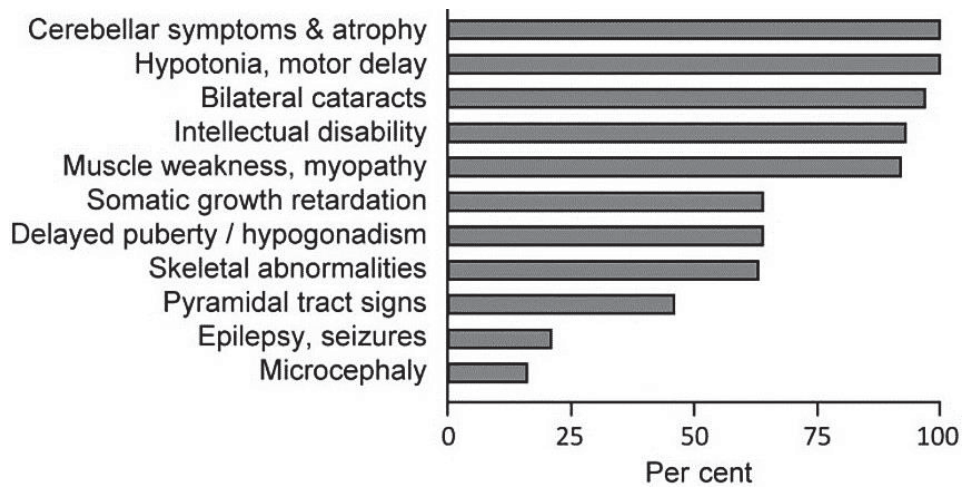

Figure 1-7. The relative frequencies of signs and symptoms associated with MSS.

Reproduced with permission from Oxford University Press. Krieger M, Roos A, Stendel C, Claeys KG, Sonmez FM, Baudis M, et al. SIL1 mutations and clinical spectrum in patients with Marinesco-Sjögren syndrome2013 2013-10-30 00:00:00. 
detailed ultrastructural study by Goto and colleagues further implicated the role of the autophagy-lysosomal system in the pathogenesis of the MSS-myopathy, indicating an ongoing autophagic phenomenon and hinting at possible metabolic defects in MSS (81). They further suggested that myofibrillar degeneration accompanied by autophagy and rimmed vacuole formation might be the primary reason for the observed muscle weakness in MSS, in addition to reporting type I fiber predominance, whereas the rare mitochondrial abnormalities were likely to be secondary consequences (81). This double membranous structure of possible sarcoplasmic reticular origin, engulfing the nucleus, came to be recognized as a hallmark of the MSS-associated myopathy (82). However, the atrophy of skeletal muscle is a distinct feature of this disease, which spares the heart, a commonly affected system in spinocerebellar degenerative disorders (77). It has also been reported that the observed myopathy lacks a neurogenic component to it $(83,84)$. Thus, Marinesco-Sjögren Syndrome was classified as a distinct disorder, arguably varying from other disorders with a partially overlapping symptoms, such as Friedreich's ataxia, and from spinocerebellar degenerative disorders and myopathies (77).

Nearly 70 years after the first report of MSS, using homozygosity mapping, MSS was attributed to segregate with the chromosomal locus of $5 \mathrm{q} 31$, albeit with some genetic heterogeneity (85). In subsequent years, two groups independently identified mutations in the ER co-chaperone SIL1 in MSS patients, with the elimination of SAR1B, which lies in close chromosomal proximity to SIL1 within the $5 \mathrm{q} 31$ locus $(64,86)$. It was noted that a subset of these mutations led to premature truncations, which might affect SIL1's stability or the stability of its encoding mRNA. Notably, mutations affecting a serinerich region, which lead to an in-frame deletion of exon 6, are also associated with MSS, indicating an essential role of the encoded domain (64). It was postulated that disruption of SIL1 might be compensated by the presence of GRP170, the alternative BiP's cochaperone with NEF-activity, in non-affected tissues (64). Around the same time, Dr. Susan Ackerman's group reported that the genetic disruption of Sill in mice leads to an adult-onset cerebellar ataxia, characterized by Purkinje cell apoptosis, intracellular protein accumulations, and induction of the UPR (Figure 1-8) (87). Sil1-disruption by either a spontaneous insertion of an ETn retrotransposon into intron 7 or the artificial insertion of a gene-trap cassette, both of which would disrupt normal splicing and the transcript beyond exon 7, resulted in an identical cerebellar phenotype. However, the insertion of the ETn retrotransposon, which would act as a splicing acceptor, would yield a prematurely truncated protein encoded by a chimeric transcript composed of exons 1-7 of Sill, 96 nucleotides of the ETn (encoding 32 amino acids), followed by an in-frame stop codon. On the other hand, the insertion of the gene-trap construct, consisting of a splicing acceptor, a CD4 transmembrane domain for retaining the $\beta$-galactosidase in the cytosol, and a $\beta$-geo cassette for selection, would yield a rather large chimeric protein encoded by exons 1-7 of Sill fused with the gene-trap construct (87). Intriguingly, it was noted that although Sill was widely expressed in the brain, only the cerebellar Purkinje cells visibly developed protein accumulations and are vulnerable to degeneration, which does not seem to affect lobule $X$ and ventral lobule IX of the vestibulocerebellum (87). Sanderek et al. utilized homology mapping and the available crystal structures of the cytosolic homologs to model the Sill-BiP interaction. This revealed that exons 6 and 9 encoded Sill domains constitute major BiP-binding sites, whereas exon 10 encodes a 


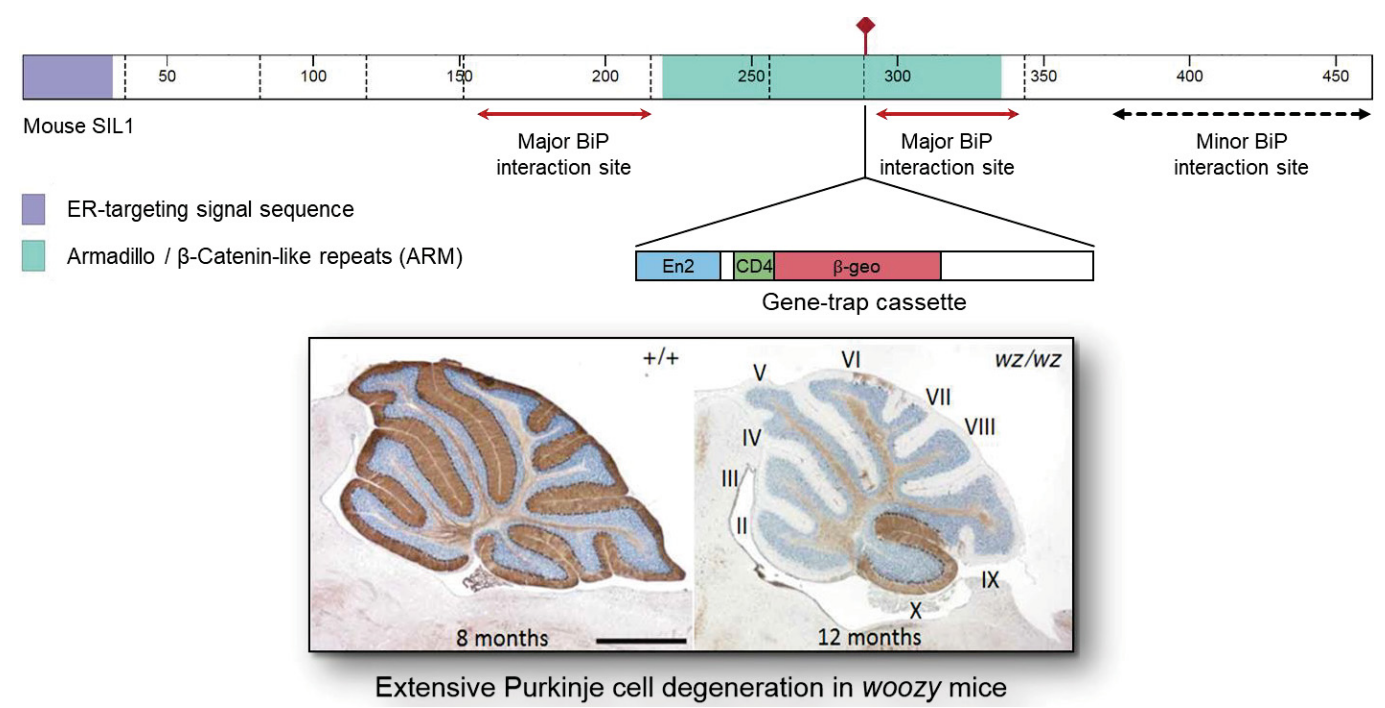

Figure 1-8. Genetic disruption of murine Sil1 phenocopies MSS symptoms.

The cartoon in the upper schematic depicts the genetic disruption of Sill in intron 7 using a gene-trap cassette. The lower panel indicates that the Sill ${ }^{\mathrm{Gt}}$ mice also develop severe cerebellar atrophy, offering great promise to study MSS in depth since they recapitulate many of the symptoms observed in this disease.

The lower panel has been reproduced with permission from Nature Publishing Group. Zhao L, Longo-Guess C, Harris BS, Lee JW, Ackerman SL. Protein accumulation and neurodegeneration in the woozy mutant mouse is caused by disruption of SIL1, a cochaperone of BiP. Nature genetics. 2005;37(9):974-9. doi: 10.1038/ng1620. PubMed PMID: 16116427. 
minor-BiP interaction site, and mutations affecting these domains are likely to affect the characteristics of the Sil1-BiP interaction (86). This model was later confirmed by structural insights into the Sil1-BiP interaction (45). Sanderek et al. further suggested that a compromised Sill would affect BiP's ATPase dependent functions, such as translocon gating or protein folding, which might cause an activation of the UPR and trigger cell death (86). These studies, together, implicate SIL1 as the causative gene in classical MSS and suggest protein folding and biosynthesis defects to be an essential part of the observed pathology in MSS.

In a follow-up study to address the genetic heterogeneity observed in MSS, Anttonen and colleagues ruled out the possibility that MSS could also be caused by mutations in either BiP (HSPA5), GRP170 (HYOU1), or amino-acyl tRNA synthetase (AARS) (88). In addition, they detected that in vitro expression of certain C-terminal SIL1 mutations, such as the Leucine to Proline conversion at residue 457 (p.L457P), results in the aggregation of SIL1 itself, signifying that SIL1 is a metastable protein and its C-terminus is particularly vulnerable to alterations in its protein sequence (88). Indeed, mutations that specifically affect the C-terminal five or six amino acids, postulated to be a divergent ER-retention motif, revealed that these changes particularly destabilize SIL1, resulting in rapid proteasomal-mediated clearance or aggregation within the ER (89). This result is in agreement with SIL1-modeling, which reveals that the very $\mathrm{C}$-terminus plays an essential role in its structural integrity rather than a role in localizing SIL1 to the ER (89). At least subset of SIL1 mutations, which retain BiP-interaction sites but yield unstable proteins, might represent promising candidates for pharmacological stabilizing agents. Additionally, in support of this hypothesis, an in-frame deletion of two amino acids (p.V231_I232del), five amino acids (p.R411_D415del), or a single amino acid substitution (p.G312R), have disastrous consequences on SIL1's stability, and either exacerbate its stability or its aggregation propensity $(78,90)$. Thus, SIL1 was identified to be the major MSS-causing gene (78).

It has been reported that the primary cause of classical MSS and an MSS-like disease in mice are loss-of-function mutations in the ER co-chaperone, SIL1, which leads to a multisystem disorder characterized by accumulation of protein aggregates, induction of the UPR, involvement of the autophagy-lysosomal system, and apoptotic cell death $(64,86,87)$. However, the underlying precise molecular events and mechanisms by which loss of a component of BiP's co-chaperone arsenal triggers the observed defects and pathologies has not been well understood. A rather challenging and complex question that remains unanswered till date is why only certain tissues or certain parts of a tissue are affected in MSS and are particularly vulnerable to imbalances in cellular protein homeostasis (proteostasis), even though SIL1 is ubiquitously expressed $(63,64)$. Furthermore, the tissues displaying the highest expression of SIL1 are not generally the most severely affected tissues in MSS or in woozy mice. In addition, it remains poorly understood why the alternative NEF for BiP, Grp170, which is also a bona fide molecular chaperone, is not able to compensate for the loss of SIL1 to an extent that is capable of preventing pathology $(44,50,53)$. Interestingly, only transgenic overexpression of Grp170 has been shown to rescue the cerebellar pathology observed in Sill-deficient mice, whereas decreasing its expression exacerbates this phenotype (91). In addition, it 
has been postulated that the cell might modulate its levels of ERdjs to achieve slower BiP's ATPase cycling, which might improve protein folding in the face of BiP's ATPase cycling deregulation due to loss of Sill. Indeed, a concomitant loss of Dnajc3/ERdj6 with that of Sil1, ameliorated ER stress and rescued neurodegeneration in Sil1-deficient mice (91). It has already been established that the two NEF's of BiP mechanistically differ in their NEF activity (65). However, functional differences in the BiP clientele they assist have largely evaded discovery. There are 46 MSS-associated mutations reported to date, a majority of which consist of frameshifts or premature truncations. However, an interesting subset results in the alteration of only a few amino acids in the native SIL1 sequence, offering the opportunity to dissect the significance of these residues in either supporting SIL1's structural integrity or functional abilities, and further inquiring if pharmacological modulators of the protein folding landscape are able to rescue structural and stability defects in the case of specific SIL1 mutations. 


\section{CHAPTER 2. SIL1, A NUCLEOTIDE EXCHANGE FACTOR FOR BIP, IS NOT REQUIRED FOR ANTIBODY ASSEMBLY OR SECRETION*}

\section{Introduction}

It has been estimated that one-third of the human genome encodes proteins that will populate the single membrane bound organelles of the cell or that will be secreted or expressed at the cell surface. These proteins are translocated into the endoplasmic reticulum (ER) lumen as they are synthesized and often undergo modifications and fold co-translationally. The proper maturation of these proteins is both assisted and monitored by the resident molecular chaperones of this organelle to prevent off-pathway folding, which might lead to aggregation, and to ensure that only those molecular forms of the newly synthesized proteins that can pass ER quality control measures are permitted to leave the ER for their proper destination $(1,92)$. Until that time, nascent proteins are retained in the ER via their interaction with molecular chaperones, and those proteins that ultimately fail to mature properly are retrotranslocated to the cytosol where they are marked for degradation by the ubiquitin proteasome system. Two major chaperone families exist in the ER; the Hsp70 family member BiP and its co-factors, and the lectin chaperones, calnexin and calreticulin and their attendant co-factors.

Like other Hsp70 family members, BiP is composed of an N-terminal nucleotide binding domain (NBD) and a C-terminal substrate binding domain (SBD) that communicate with each other via a linker region. The binding of BiP to substrates is regulated by its nucleotide bound state $(57,93-95)$. When ATP occupies the NBD, the lid of the SBD is open allowing ready access to extended regions of nascent proteins or substrates, but this open conformation does not promote stable binding. However, the binding of substrate to the SBD triggers ATP hydrolysis, which in turn induces at least partial closing of the lid over the bound substrate and stabilizes this association $(57,94)$. The substrate does not fold while bound to BiP but is protected from off-pathway intermediates or aggregation. The lid of the SBD must be re-opened to release the substrate so it can fold, which requires the bound ADP to be exchanged for ATP. Although three different structurally unrelated groups of nucleotide exchange factors (NEFs) exist in the cytosol of eukaryotic organisms, only two types, each with a single representative, have been identified in the ER: the HSPBP1 orthologue, BAP/SIL1 (63, 96), and the large Hsp70 member, Grp170 $(48,50)$. While SIL1 seems to only function as a NEF, the large Hsp70 proteins, including Grp170, also bind directly to substrates $(44,54,97,98)$, making their functional activity more complex and currently less clear. Structural analysis of yeast Sillp reveals that it binds to the NBD of BiP and disrupts a

\footnotetext{
*Reprinted from final submission with permission. Ichhaporia, V. P., Sanford, T., Howes, J., Marion, T. N., \& Hendershot, L. M. (2015). Sil1, a nucleotide exchange factor for $\mathrm{BiP}$, is not required for antibody assembly or secretion. Molecular Biology of the Cell, 26(3), 420-429.
} 
hydrogen bond between the two lobes, which results in a rearrangement of the NBD that induces the release of ADP from BiP (45). Based on homology mapping with the cytosolic orthologue HspBP1, exons 6 and 9 of SIL1 are predicted to constitute the major interaction site with the NBD of BiP with exon 10 providing a minor interaction (86).

Mutations in the SIL1 gene have been found in over half of the cases of Marinesco-Sjögren syndrome (MSS) $(64,86)$, an autosomal recessive disease characterized by multi-system defects including ataxia due to Purkinje cell loss, cataracts, and progressive myopathy $(76,78)$. MSS-associated mutations occur throughout the SIL1 gene and most lead to the disruption of significant portions of the protein $(88,99)$, although the fate of most of these fragments has not been well-characterized. In fact, it remains unclear whether the associated pathologies arise from reduced maturation of essential secretory pathway proteins, depletion of BiP stores resulting in activation of the ER stress response, toxicity due to aggregation of secretory pathway proteins, or even perhaps of the mutant SIL1 protein itself. Three MSS-associated mutations have been identified that disrupt only the last 4 or 5 amino acids, which were shown to reduce the solubility of the mutant proteins and decrease their stability leading to significantly diminished expression of mutant SIL1 $(88,89)$. Although a SIL1-null mouse is not available, the gene has been disrupted spontaneously by transposon insertion, Sill ${ }^{\mathrm{wz}}$, and by gene-trap methodology, Sill ${ }^{\mathrm{Gt}}(87)$. In both cases, the Sill gene is disrupted between exons 7 and 8, resulting in loss of amino acids 261-465 of the SIL1 protein. The resulting mice are referred to as woozy mice and have been reported to phenocopy some of the pathologies associated with MSS including cerebellar loss resulting in ataxia (87) and a progressive myopathy (100). Examination of cerebella from these mice revealed the presence of protein aggregates and activation of the ER stress response, which is induced by the accumulation of unfolded proteins in this organelle.

To begin to understand the effects of Sill gene disruption on secretory pathway protein maturation, we chose to examine the assembly and secretion of immunoglobulins, which are the best studied $\mathrm{BiP}$ substrates $(20,21,101-103)$. Immunoglobulin is a heteromeric protein composed of two heavy and two light chains (HC and LC, respectively) that are combined in the ER. BiP binds to all the assembly intermediates and prevents their secretion, but does not interact with completely assembled complexes $(21,102)$. Co-expression of BiP mutants that cannot be released from Ig heavy chains with ATP prevents the assembly and secretion of antibodies $(57,104)$, suggesting a NEF might be required for this process normally. Lastly, we considered that the incredibly large quantities of antibodies produced by plasma cells, which puts particularly high demands on ER homeostasis, would make these cells particularly sensitive to disruptions in the chaperone network. Together these points argue that antibodies would represent a good model to examine the requirements for a nucleotide exchange factor in BiPmediated folding. The availability of a mouse model with a disrupted Sill gene and Epstein-Barr virus (EBV) transformed B lymphoblastoid lines (LBLs) from individuals with MSS provide important biological tools for examining the effect of SIL1 protein loss on antibody assembly and secretion both in vivo and ex vivo, which in addition to establishing the requirement for SIL1 in Ig assembly and secretion could also shed light on humoral immune function in patients. 


\section{Results}

\section{Detection of disrupted Sil1 transcripts in woozy mice}

The Sill gene has been disrupted beyond exon 7 in woozy mice by either a spontaneous insertion of an ETn retrotransposon, Sill ${ }^{\mathrm{wz}}$, or the insertion of the $\beta$-geo gene-trap cassette, Sill $^{\mathrm{Gt}}$ (87). In both cases, exon 7 is spliced to the inserted gene generating a fusion protein that should possess the N-terminal 260 amino acids (out of a total 465 amino acids) encoded by the Sill gene followed by either 32 amino acids of the transposon or a CD4 transmembrane region and a $\beta$-geo cassette, respectively (Figure 2-1). In spite of the very different fusion proteins generated in these two woozy mice, the phenotypes appear to be very similar suggesting both may lead to a loss of functional SIL1 protein. Importantly, the chimeric product of neither SIL1 disruption has been examined, but a truncated version of SIL1 possessing only the N-terminal 260 amino acids was expressed in COS-1 cells. This mutant is less stable and binds BiP with a reduced affinity compared to the wild-type SIL1 protein (87). As Sill expression had not been previously measured in plasmablasts and previous reports suggest that levels can vary significantly between various tissues in humans (63) and mice (105), we first examined SIL1 transcript levels in day 4 LPS stimulated splenic B cells from wild-type and woozy mice by real-time PCR. Primers were selectively designed to amplify regions that are either shared by the Sill ${ }^{\mathrm{WT}}$ and Sill $^{\mathrm{Gt}}$ transcripts (primer pair 1) or are present exclusively in Sil1 ${ }^{\mathrm{WT}}$ transcripts (primer pair 2) (Figure 2-1). For reasons that are unclear, we found that $S_{i l 1}{ }^{\mathrm{Gt}}$ transcripts were present at only a third of the level of the wild-type SIL1 transcripts. As expected, the SIL1 transcripts from the Sill ${ }^{\mathrm{Gt}}$ mouse could not be amplified with the primer pair extending across exons 9 and 10, demonstrating that the plasmablasts are indeed derived from $\mathrm{Sill}^{\mathrm{Gt}}$ homozygous mice as opposed to mis-genotyped heterozygous mice. All mice used for immunizations and LPS stimulation assays were genotyped at least three times to ensure accuracy of genotypes being used.

\section{Characterization of immune populations in wild-type and woozy mice}

We first examined the effects of Sill disruption on the distribution of cellular subpopulations present in the spleen and thymus. The relative percent of $\mathrm{CD}^{+} / \mathrm{CD}^{+} \mathrm{T}$ helper and $\mathrm{CD}^{+} / \mathrm{CD}^{+}$cytotoxic T-lymphocytes, B-lymphocytes $\left(\mathrm{B} 220^{+}\right)$, macrophages $\left(\mathrm{Mac}^{+}\right)$, granulocytes $\left(\mathrm{Gr}^{+}\right)$and natural killer cells $\left(\mathrm{NK} 1.1^{+}\right)$were very similar between wild-type and Sill ${ }^{\mathrm{Gt}}$ mice (Figure 2-2A). Thymocytes were stained for a variety of markers to identify the various developmental stages of T-cell populations in the Sill ${ }^{\mathrm{Gt}}$ mouse compared to their wild-type littermates (Figure 2-2B, C). We observed no significant helper and $\mathrm{CD}^{+} / \mathrm{CD}^{+}$cytotoxic T-lymphocytes, B-lymphocytes $\left(\mathrm{B} 220^{+}\right)$, macrophages $\left(\mathrm{Mac}^{+}\right)$, granulocytes $\left(\mathrm{Grl}^{+}\right)$and natural killer cells $\left(\mathrm{NK} 1.1^{+}\right)$were very similar between wild-type and Sil1 ${ }^{\mathrm{Gt}}$ mice (Figure 2-2A). Thymocytes were stained for a variety of markers to identify the various developmental stages of T-cell populations in the Sill ${ }^{\text {Gt }}$ mouse compared to their wild-type littermates (Figure 2-2B, C). We observed 


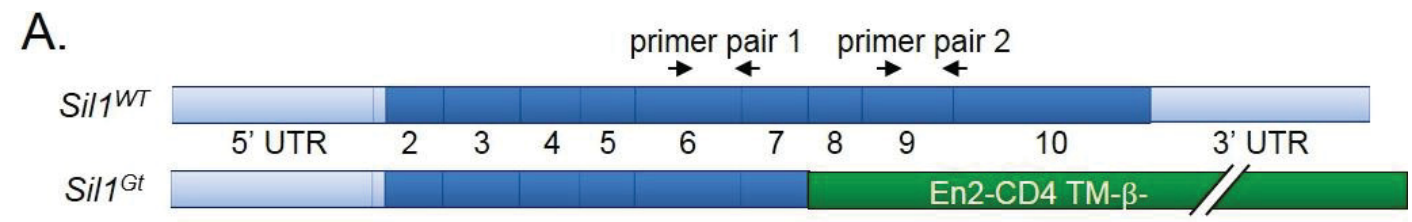

B.

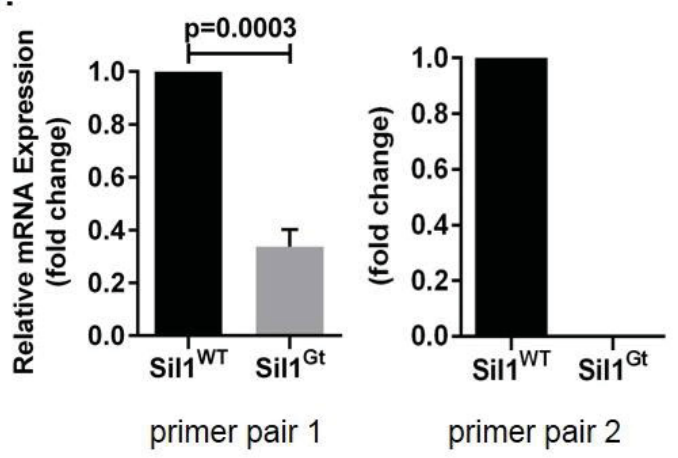

C.

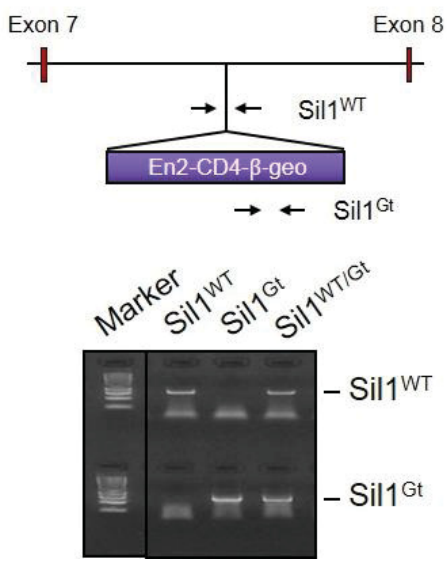

Figure 2-1. Schemes for quantifying SIL1 mRNA and genotyping mice.

(A) Schematic of expected SIL1 transcripts produced in Sill ${ }^{\mathrm{WT}}$ and Sill ${ }^{\mathrm{Gt}}$ mice with location of the primer pairs used for amplifying cDNA. (B) The relative mRNA levels of SIL1 in day 4 LPS-stimulated splenic B cells were analyzed by real-time PCR with the indicated primer pairs. The region amplified by primer pair 1 is shared between Sill ${ }^{\mathrm{WT}}$ and $\mathrm{Sill}^{\mathrm{Gt}}$ transcripts, whereas the product of primer pair 2 is exclusively present in Sill ${ }^{\mathrm{WT}}$ transcripts; thus Sill ${ }^{\mathrm{Gt}}$ transcripts are not detected. (C) Schematic displaying the insertion of the $\beta$-geo gene-trap cassette into intron 7 (not to scale) with location of primer pairs used for genotyping mice. 

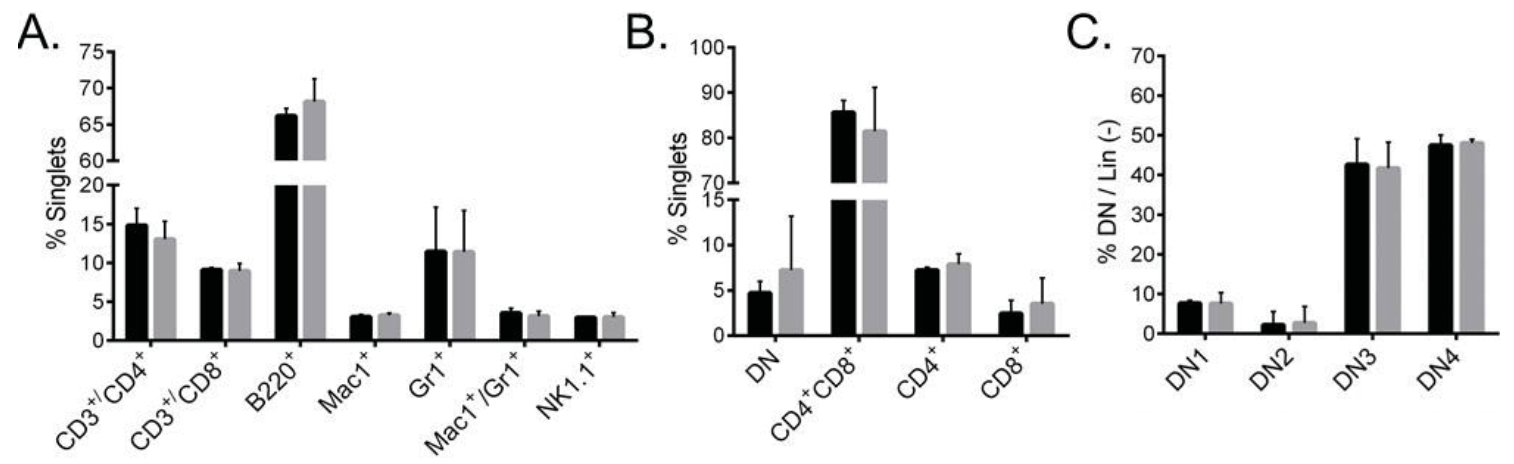

Figure 2-2. Disruption of Sill gene does not affect splenocyte or thymocyte populations.

(A) Single cell splenocytes from wild-type (black bars) and woozy mice (gray bars) were stained with the indicated antibodies, subjected to FACS analyses, and expressed as a percent of nucleated, singlet splenic cells. (B, C) Thymocytes from wild-type and woozy mice were stained to identify developmental subpopulations and similarly portrayed.

Error bars indicate means \pm s.d. 
no significant differences in the numbers of any of the developmental stages of T-cells. Thus, disruption of the Sill gene did not appear to affect the maturation or survival of any of the indicated immune populations.

\section{Sil1 ${ }^{\mathrm{Gt}}$ mice produce normal levels of antigen-specific antibodies in response to immunization with a T-dependent antigen}

Although mature B and T cell subpopulations in the woozy mouse appeared normal, we examined their ability to respond to immunization with the T-dependent antigen, $\mathrm{NP}_{15}$-KLH. Ten wild-type and ten woozy mice were immunized and boosted 2

weeks later. Sera were obtained at the indicated days post-immunization, and $\mathrm{NP}_{15}-\mathrm{KLH}$ specific antibodies were quantified to detect differences in either the magnitude or kinetics of antibody production. We chose to measure three antibody isotypes, including two that assemble via the $\mathrm{H} \rightarrow \mathrm{HL} \rightarrow \mathrm{H}_{2} \mathrm{~L}_{2}$ pathway (IgM and $\mathrm{IgG} 2 \mathrm{~b}$ ) and one that assembles through $\mathrm{H} \rightarrow \mathrm{H}_{2} \rightarrow \mathrm{H}_{2} \mathrm{~L} \rightarrow \mathrm{H}_{2} \mathrm{~L}_{2}$ intermediates (IgG1). Since heavy chains remain incompletely folded until they associate with light chains (101), we reasoned that antibodies assembling through the latter pathway might be more dependent on BiP and as a result show a greater need for functional SIL1. Somewhat surprisingly, we found that there was no significant difference in either the magnitude or kinetics of producing $\operatorname{IgM}$, $\mathrm{IgG}_{2}$ b, or IgG1 antibodies to $\mathrm{NP}_{15}-\mathrm{KLH}$ (Figure 2-3). This argued that the loss of SIL1 function did not prevent the secretion of antibodies regardless of their route of assembly.

\section{LPS-stimulated B cells from Sil1 ${ }^{\mathrm{Gt}}$ mice synthesize and secrete IgM antibodies similar to wild-type littermates}

We next examined Ig synthesis in purified murine splenic B cells stimulated with LPS ex vivo. This allowed us to directly examine Ig secretion over a shorter time span and to determine whether unassembled immunoglobulin chains accumulated in the woozy splenocytes. Isolated splenic B cells were cultured with LPS, and cells and culture supernatant were isolated every day, for four days. There was no significant difference in the growth rate of stimulated B cells from woozy mice compared to their wild-type littermates (data not shown). Western blot analysis of cell lysates revealed robust up-regulation of the secretory type of IgM heavy chains $\left(\mu_{\mathrm{s}}\right)$ and $\kappa$ light chain in both wild-type and woozy mice to very similar levels (Figure 2-4A). When culture supernatants were analyzed for secreted $\operatorname{IgM}$, similar levels of $\mu$ heavy and $\kappa$ light chains were readily detected after two days of stimulation and continued to increase throughout the course of the experiment in both cases (Figure 2-4B). We determined the assembly status of IgM present in the splenocytes after 3 and 4 days of LPS stimulation on nonreducing gels. Under steady state conditions, the $\mu$ heavy chains in the cells from both wild-type and woozy mice were present predominantly as $\mathrm{H}_{2} \mathrm{~L}_{2}$ monomers and $\mathrm{H}_{2} \mathrm{~L}_{2}$ polymers, which are likely to be pentamers, although a polymeric intermediate can be detected with both anti- $\mu$ and anti- $\kappa$ antisera (Figure 2-4C). Trace amounts of HL hemimers and LC dimers were detected. However, there was no evidence that unassembled or incompletely assembled Ig molecules accumulated in plasmablasts from 
A.

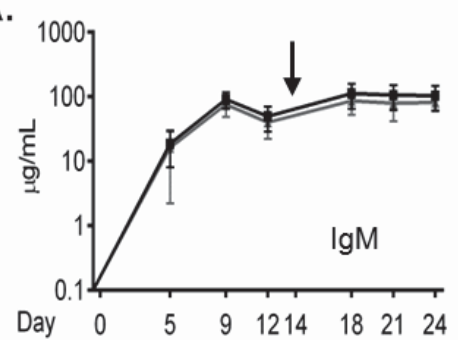

B.

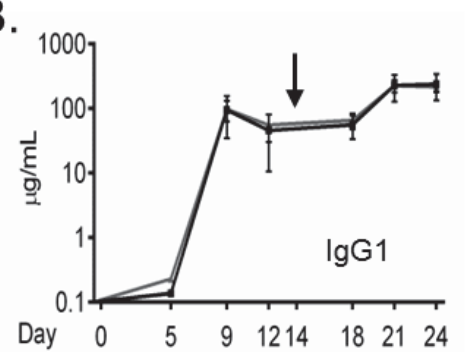

C.

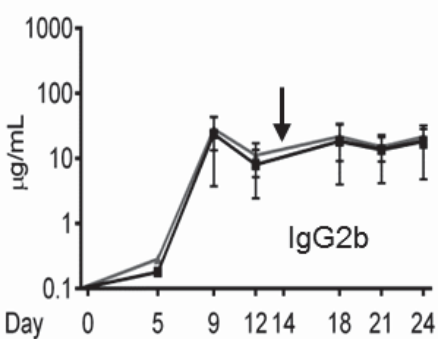

Figure 2-3. $\quad$ Sill $^{\mathrm{Gt}}$ mice respond to $\mathrm{T}$-dependent antigens similarly to age-matched Sill $^{\mathrm{WT}}$ mice.

Sill $^{\mathrm{WT}}$ (black line) and Sill ${ }^{\mathrm{Gt}}$ (gray line) mice were challenged with $\mathrm{NP}_{15}-\mathrm{KLH}$ as described, and serum was collected at the indicated intervals. Red arrow indicates the booster immunization. Antigen-specific responses comprised of IgM (A), IgG1 (B) and IgG2b (C) were determined by ELISA. Each point displays the median value. $N=10$ Sill ${ }^{\mathrm{WT}}$ and Sill $^{\mathrm{Gt}}$ mice each. Error bars indicate interquartile range of values. 
A.

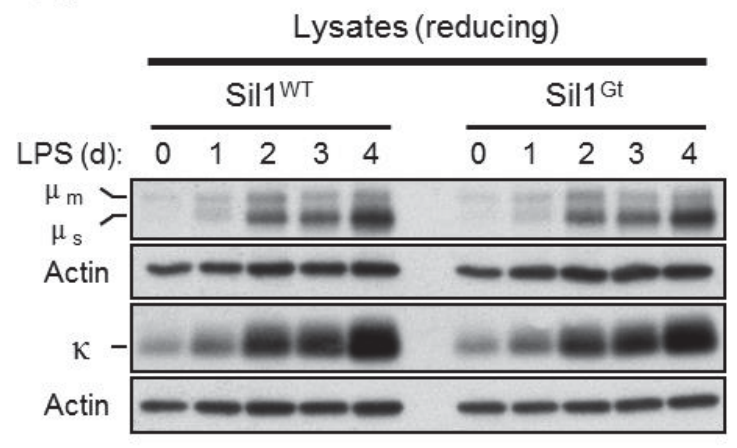

B.

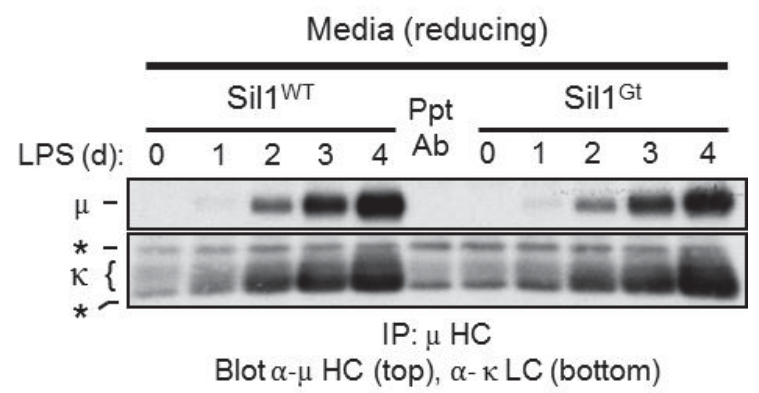

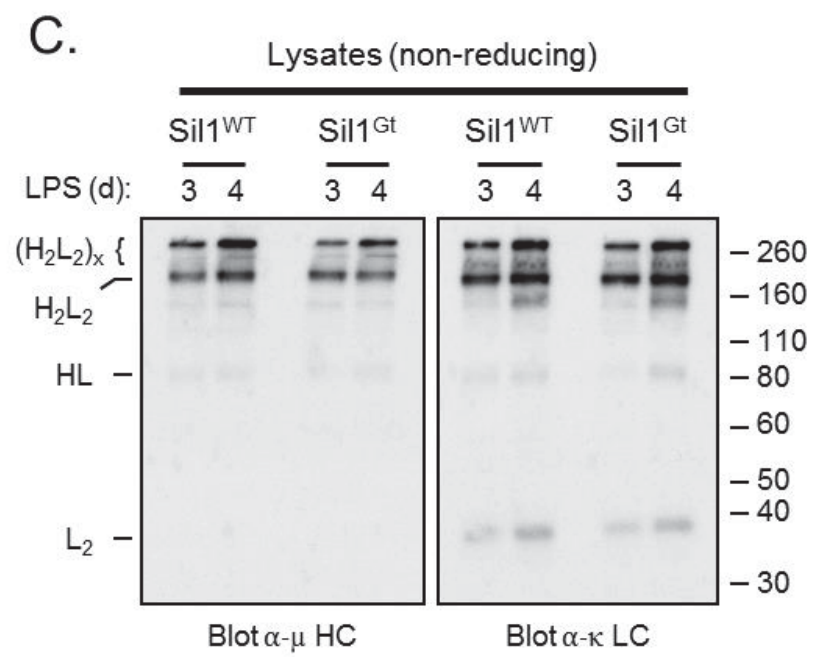

Figure 2-4. Disruption of Sil1 gene does not adversely affect IgM biosynthesis or secretion in response to ex vivo LPS stimulation.

Isolated splenocytes were cultured with LPS for the indicated times, and lysates corresponding to equivalent numbers of cells were analyzed under reducing (A) or nonreducing $(\mathrm{C})$ conditions followed by blotting with the indicated immune reagents. Actin served as a control for loading. (B) Culture supernatants corresponding to an equivalent number of cells for each day were immunoprecipitated with anti- $\mu \mathrm{HC}$ antibody, analyzed by reducing SDS-PAGE, and probed with the indicated antibodies. The center lane contains only the immunoprecipitating antibody (Ppt Ab), which is recognized by the secondary antibody used to identify the mouse $\kappa$ LC. Its mobility is designated with asterisks on the left. 
the woozy mice to a greater extent than observed in the wild-type cells. To assess whether unassembled IgM was aggregating in these cells, we examined the NP-40 insoluble fraction for the presence of IgM. Very small amounts of insoluble $\kappa$ light chains and $\mu$ heavy chains could be detected in the differentiating splenic B cells, but the levels of both were very similar in the two genotypes (Figure 2-5A). Thus, we found no evidence that the woozy mice were compromised in their ability to assemble or secrete antibodies, suggesting that SIL1 was not required to release BiP from immunoglobulin heavy chains.

\section{Sil1 disruption does not affect chaperone levels in LPS stimulated B cells}

Our examination of day 4 LPS-simulated splenic B cells revealed that SIL1 transcripts were present in wild-type cells and in lesser amounts in the woozy cells (Figure 2-1). Most ER molecular chaperones and resident proteins are normally upregulated during plasma cell differentiation $(106,107)$. This occurs via activation of a limited UPR $(108,109)$, which serves to maintain homeostasis in the dramatically expanded ER in preparation for the massive production of antibodies. However, since Sill is not induced by the UPR (63), we considered the possibility that the SIL1 protein expression remained unchanged during plasma cell differentiation and therefore did not contribute significantly to BiP-mediated folding in these cells. To address this possibility, we examined SIL1 protein levels in the wild-type and woozy mice over the course of LPS-induced differentiation. Although basal levels were quite low in the unstimulated splenic B cells, SIL1 protein became readily detectable over the course of differentiation in the wild-type plasmablasts (Figure 2-6A). However, when the differentiating woozy splenocytes were examined with this antibody, we were unable to observe a unique band in the region corresponding to the predicted molecular weight for the chimeric Sill ${ }^{\mathrm{Gt}}$ protein (Figure 2-5B).

The loss of SIL1-function in mouse Purkinje cells leads to activation of the unfolded protein response (UPR), which results in higher than normal levels of some ER chaperones including Grp170, and eventually to cell death (87). Both of these consequences could be prevented by transgenic over-expression of Grp170 (91), which has both nucleotide exchange $(48,50)$ and chaperone activity $(44,110)$. Thus, we wished to determine if the loss of SIL1 might be compensated by an even greater increase in expression of Grp170 or other chaperones during plasma cell differentiation. When cell lysates from wild-type and woozy LPS-stimulated splenic B cells were examined, the upregulation of Grp170 and other chaperones like Grp94, calnexin, and calreticulin, all of which have been reported to bind $\mu$ heavy chains $(97,111,112)$, appeared to be very similar (Figure 2-6A, B). Thus, unlike the Purkinje cells of the woozy mouse, there was no compensating over-expression of Grp170 or any other ER chaperone examined.

The ER also possesses a number of DnaJ-like co-factors that serve to bind unfolded protein substrates and deliver them to BiP (55). A previous study reported that deletion of one of the ER-localized ERdj proteins in the Sill $1^{\mathrm{Gt}}$ mice partially suppressed the Purkinje defects associated with Sill disruption (91), perhaps due to decreased 
A.

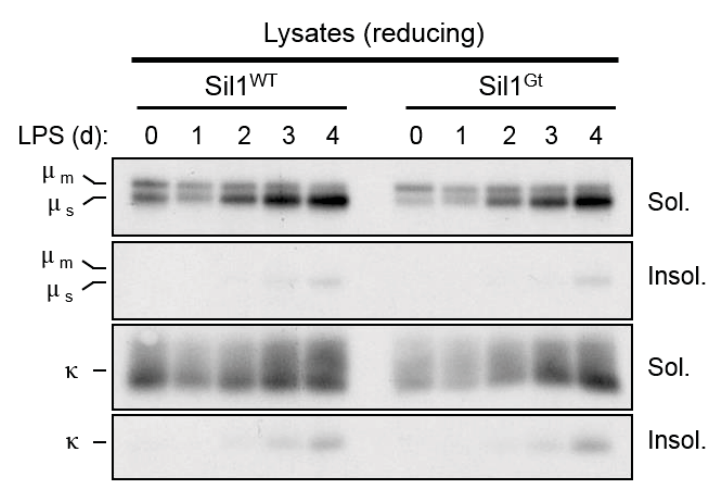

B.

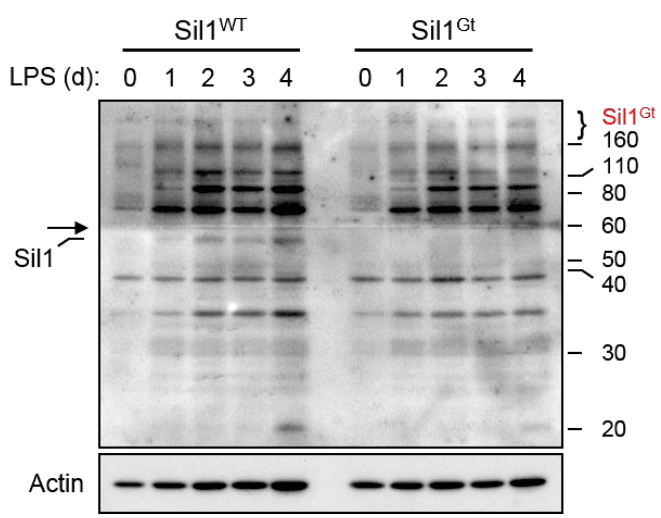

Figure 2-5. Disruption of Sill does not increase Ig heavy or light chain aggregation.

(A) NP-40 insoluble proteins were solubilized in SDS and equivalent fractions of the SDS-solubilized (Insol.) and NP-40 soluble (Sol.) material were electrophoresed under reducing conditions and probed with the indicated antibodies. Exposure times for NP-40 soluble and insoluble material are identical. (B) Full membrane of the SIL1 panel used in Figure 2-4A probed with an anti- SIL1 antibody from Proteintech. The region corresponding to the predicted molecular weight for the Sill ${ }^{\mathrm{Gt}}$ protein is indicated. Note the membrane was previously cut for probing with different antibodies. The cut line is indicated with an arrow. 
A.

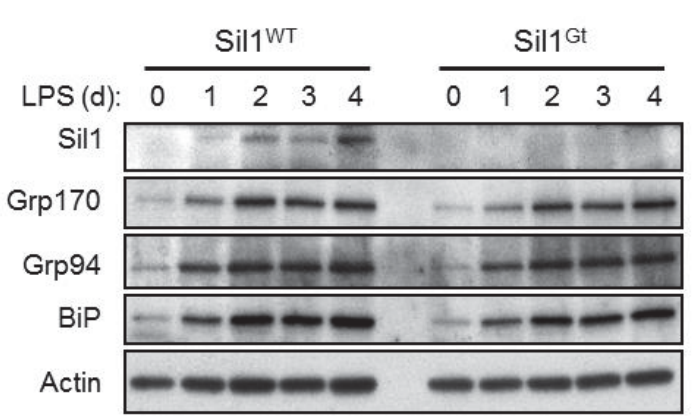

B.

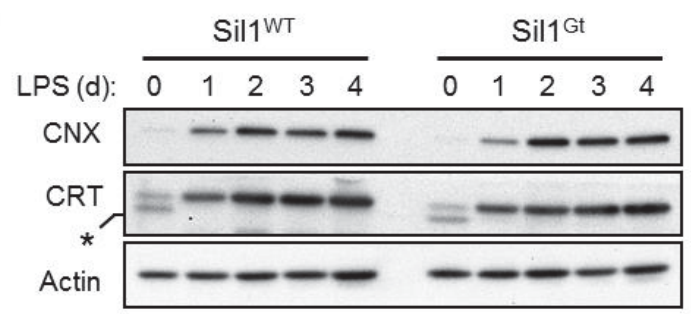

C.

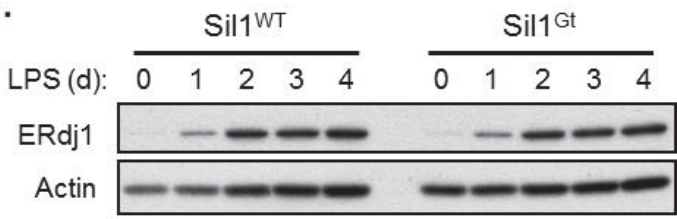

D.

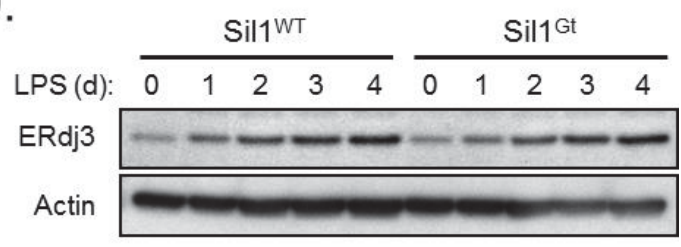

E.

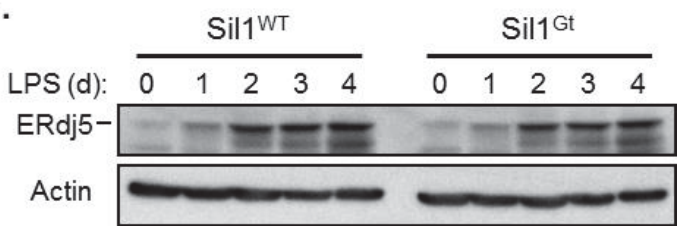

Figure 2-6. Chaperone levels are unaltered during plasmablast differentiation in Sil1 ${ }^{\mathrm{Gt}}$ mice.

Direct cell lysates were prepared, electrophoresed under reducing conditions as described in Figure 3, and probed with the indicated antibodies to molecular chaperones $(\mathrm{A}, \mathrm{B})$ and $\mathrm{BiP}$ co-factors (C-E). As samples were run on different gels a loading control is included for each. The anti- SIL1 antibody used in panel (A) was purchased from Proteintech. The anti-calreticulin antibody (CRT) used in panel B detected an additional unknown band only in the unstimulated splenocytes, which is indicated with an asterisk. 
transfer of substrates to BiP. Therefore, we compared levels of several of the resident ER DnaJ proteins during differentiation. All three ERdj proteins examined were similarly up-regulated in wild-type mice and their woozy littermates in response to plasma cell differentiation (Figure 2-6C-E). This includes ERdj3, which is specific for Ig heavy chains (113) and ERdj5, which also interacts with unassembled Ig heavy chains (our unpublished data). Thus, there is no evidence that the loss of SIL1 function is compensated by increasing Grp170 levels or decreasing these ERdj BiP co-factors.

\section{Immunoglobulin synthesis is not relegated to a different chaperone system in SIL1 disrupted splenic B cells during differentiation}

Although we saw no evidence that woozy mice compensated for the loss of SIL1 function by abnormally up-regulating Grp170 or altering the expression of other ER resident proteins, it was conceivable that in the absence of SIL1, immunoglobulin synthesis was controlled by another ER chaperone. Notably, Grp94 (112) and Grp170 $(44,97)$ can bind directly to immunoglobulin chains as a chaperone, and the lectin chaperones have also been demonstrated to interact with $\mu$ heavy chains (111). To determine if IgM antibodies were relying on another chaperone for its assembly, we performed pulse-chase experiments on splenic B cells that were stimulated with LPS for 2 days and prepared cell lysates under conditions most conducive to maintaining association of client proteins for each of the chaperones. For BiP and Grp170, lysates were prepared with apyrase or Mg2+-ATP respectively, while for Grp94, calnexin and calreticulin no supplements were added. When the pulse-labeled samples were examined (Figure 2-7 A, B) there was no evidence of $\mu$ heavy chains co-precipitating with Grp170, Grp94, calnexin or calreticulin in either genotype above the background observed with Protein A agarose, and consequently no $\mu$ heavy chains were co-isolated with the chaperones immunoprecipitated from chase samples (Figure 2-7C, D). This strongly argues that IgM antibodies were not utilizing one of these chaperones in the Sill $^{\mathrm{Gt}}$ cells. The fact that $\mu$ heavy chains and BiP migrate identically on these gels made it impossible to determine if the heavy chains were interacting with $\mathrm{BiP}$ in both genetic backgrounds, although the signal in the $\mathrm{BiP}$ immunoprecipitated material appeared slightly more sharp and less strong in the chase sample compared to the pulse. To determine if $\mu$ heavy chains were interacting with BiP in the Sill ${ }^{\mathrm{Gt}}$ cells, we performed immunoprecipitation coupled to western blotting assays. We found the secretory form of $\mu\left(\mu_{\mathrm{s}}\right)$ coimmunoprecipitated with $\mathrm{BiP}$, and correspondingly BiP was co-isolated with anti- $\mu$ precipitated material in plasmablasts from both wild-type and woozy mice (Figure 2-8). Thus, although SIL1 was dispensable for IgM production, it was not because BiP was not used during Ig assembly in the Sill ${ }^{\text {Gt }}$ cells. Together these data demonstrate there is no evidence antibodies are dispatched to a different chaperone system when SIL1 is not available to release $\mathrm{BiP}$ from $\mathrm{Ig}$ heavy chains nor do unassembled heavy chains remain bound to $\mathrm{BiP}$ in these cells. 

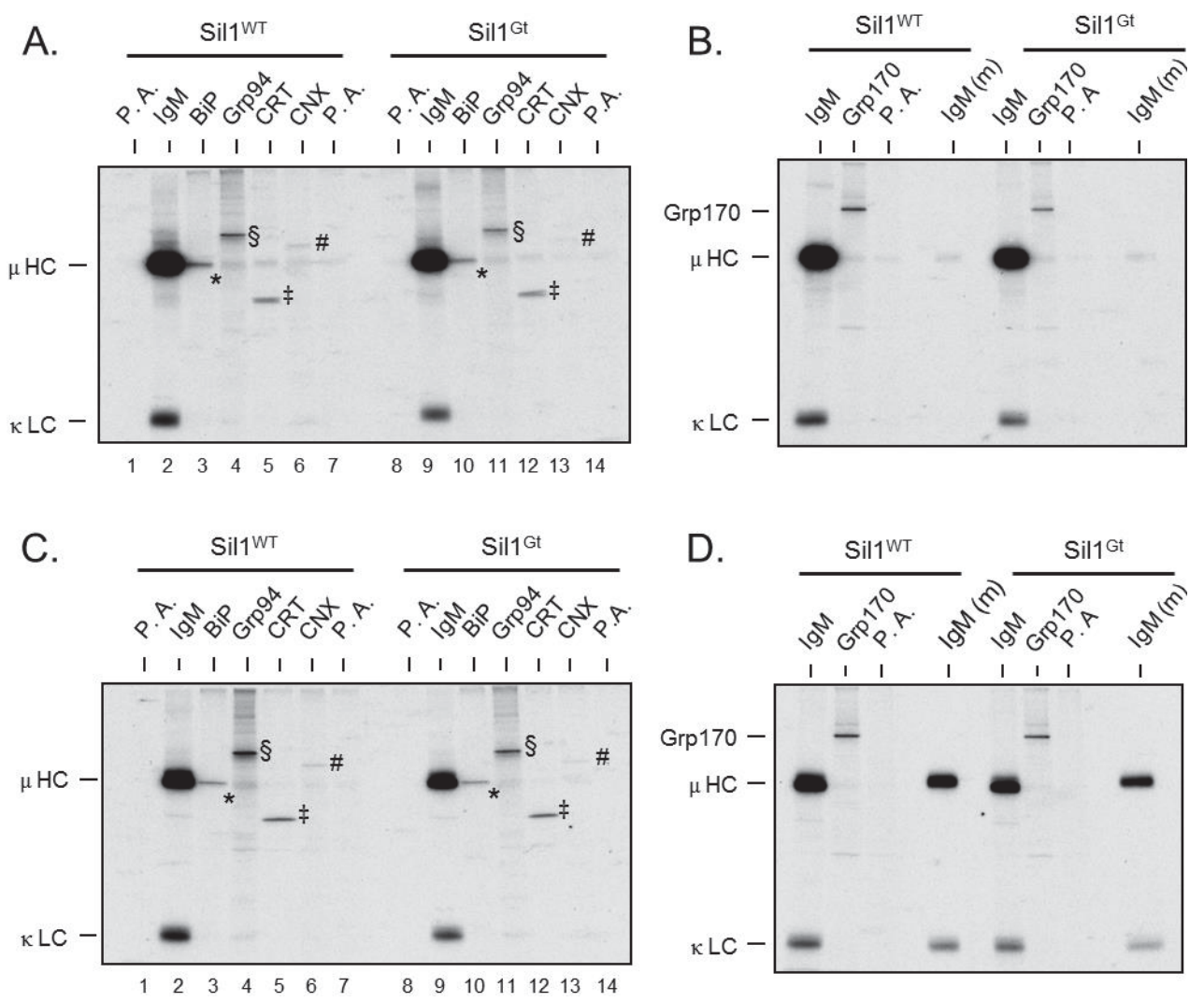

Figure 2-7. Loss of SIL1 does not redirect IgM assembly to another chaperone system.

LPS stimulated splenic B cells from day 2 were pulse labeled with ${ }^{35} \mathrm{~S}$ methionine and cysteine and chased for 0 or 1 hour. (A,C) Aliquots of the cells were lysed with apyrase (lanes 1-3 and 8-10), without apyrase (lanes 4-7 and 11-14), or (B,D) with the addition of $\mathrm{Mg}^{2+}$-ATP (lanes 1-3 and 5-7). The lysates and media (m) were immunoprecipitated with the indicated antibodies and analyzed by reducing SDS-PAGE. The migration of these chaperones are denoted with the following symbols; BiP (*), Grp94 (§), calreticulin $(\$)$, and calnexin $(\#)$, and mobility of heavy and light chains are indicated. 
A.

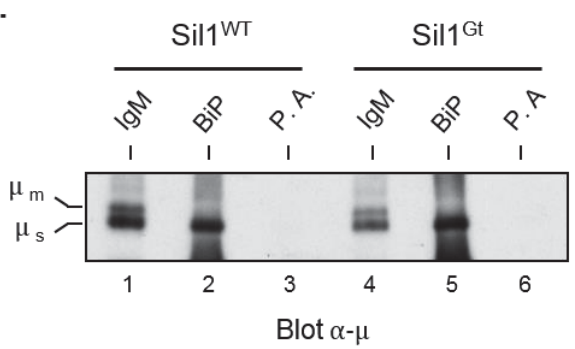

B.

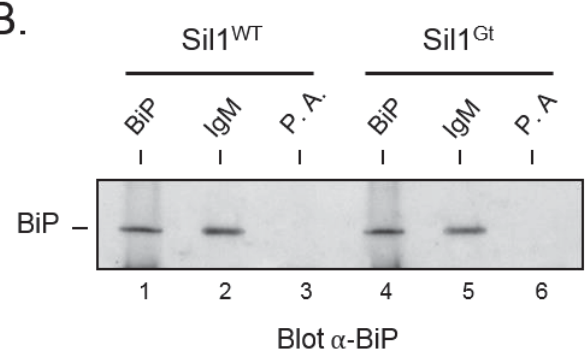

Figure 2-8. Disruption of SIL1 does not alter BiP's interaction with the Ig heavy chain.

Splenic B cells were stimulated with LPS for 2 days and lysed in the presence of apyrase. Cell lysates were immunoprecipitated as indicated, separated on reducing SDS-gels, and blotted with antibodies against $\mu \mathrm{HC}(\mathrm{A})$ or $\mathrm{BiP}(\mathrm{B})$. For lanes 1 and 4 in both cases, 1/10 the amount of lysate was immunoprecipitated compared to lanes 2, 3, 5, and 6. 


\section{Antibody assembly and secretion are normal in lymphoblastoid lines from individuals with MSS}

We were surprised to find that there was no aspect of antibody production in vivo or ex vivo that was affected by SIL1 disruption, even though these woozy mice have profound defects in a variety of other tissues $(91,100,105)$. Since the immune systems of mice and man do not always function identically (114), we obtained lymphoblastoid lines produced from three related individuals with MSS and two non-affected relatives (115). In sequencing genomic DNA to verify the alteration of the Sill gene, we discovered a novel mutation in the 5' GT-rich region of the splice donor site of intron 9 (c.1029+6T $>$ C) in addition to the previously reported c.1030-18G $>$ A mutation, 18 bases upstream of exon 10 (115) (Figure 2-9A). Sequencing of cDNA confirmed that this resulted in aberrant splicing of exon 9 causing a frameshift after Lys 343 and a premature stop 13 amino acids later (Figure 2-9B). Western blot analyses of these lines revealed that all three of the MSS cell lines produced both heavy and light chains, which would allow us to examine Ig assembly and secretion (Figure 2-9C). Using both our polyclonal antiserum raised to the full-length SIL1 protein and an antibody that was raised to a region of SIL1 that is retained in the predicted MSS protein, we were unable to detect the mutant SIL1 protein under steady state conditions in any of the MSS lines (Figure 2-10A), suggesting that either the mutant protein is not readily translated or is unstable. To test this possibility, a control and MSS patient-derived lymphoblastoid cell lines were incubated with and without MG132 to prevent proteasomal degradation. A faint band corresponding to the predicted size of the SIL1 mutant protein could be detected only after inhibiting degradation indicating that the truncated MSS-associated protein was unstable (Figure 2-10B). There was no visible change in the expression of Grp170, BiP or calreticulin (Figure 2-10A), which is in keeping with our data from the LPSstimulated murine plasmablasts (Figure 2-5). To examine Ig assembly and secretion, we chose MSS lines \#1 and \#2, which both produced IgG antibodies that assemble through the $\mathrm{H} \rightarrow \mathrm{H}_{2} \rightarrow \mathrm{H}_{2} \mathrm{~L} \rightarrow \mathrm{H}_{2} \mathrm{~L}_{2}$ pathway, as well as one of the control lines that used the same Ig assembly pathway. Pulse-chase experiments revealed that both MSS cell lines were able to readily assemble and secrete IgG antibodies and that there was no evidence of incompletely assembled precursor molecules accumulating in the cells (Figure 2-11). Together, these data indicate that SIL1 protein/function is not required for BiP release from antibodies during their production.

\section{Discussion}

\section{SIL1 is not required for Ig assembly or secretion}

Mutations in SIL1 in individuals with Marinesco-Sjögren syndrome results in multiple system defects $(84,99,116,117)$, many of which are phenocopied by disrupting this gene in mice $(87,100)$. To begin to understand the consequences of losing SIL1 function on the release of substrates from BiP, we turned to immunoglobulins, which can be studied in vivo and ex vivo, allowing greater scrutiny of the process. Surprisingly, we 
A.

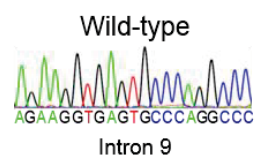

MSS \#1-3

c. $1029+6 T>C$

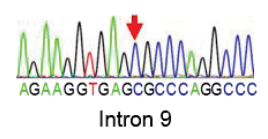

B.
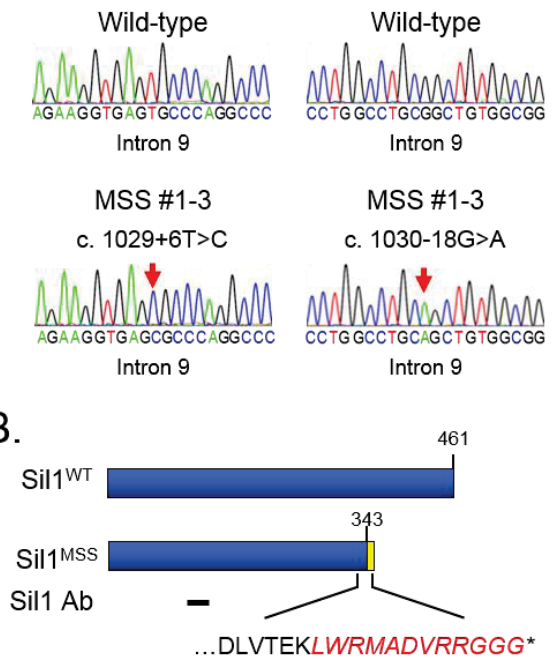

MSS \#1-3

c. $1030-18 \mathrm{G}>\mathrm{A}$
C.
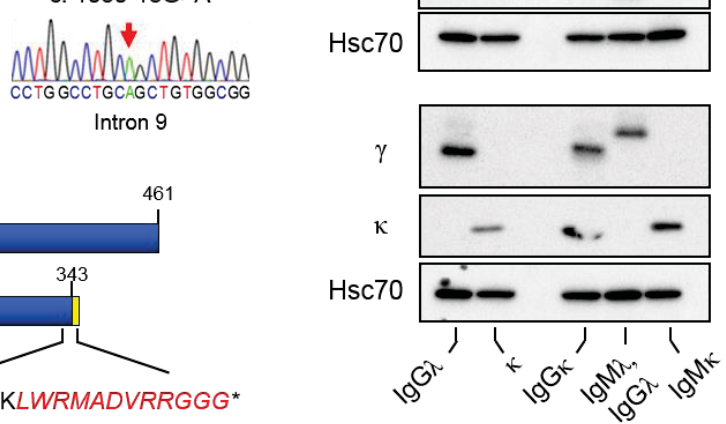

Figure 2-9. Characterization of Lymphoblastoid cell lines from normal controls and MSS affected individuals.

(A) Sequencing of the Sill gene revealed a previously unreported substitution (c.1029+6T $>$ C) in intron 9 (left panel) in addition to the reported homozygous mutation (c.1030-18G $>$ A) in this intron, which creates an aberrant splice acceptor site (right panel) in these MSS patients. The electropherograms of a wild-type control (top) and an affected individual (bottom) are shown. (B) Schematic representation of the region affected by the mutations on the SIL1 protein (blue), including the non-related amino acids resulting from the frameshift (yellow), which are listed below in red and italicized. The bar under the schematics indicates the portion of the protein used by LifeSpan Biosciences to generate the anti- SIL1 antibody, which is retained in this SIL1 mutant protein. (C) Direct cell lysates from the indicated LBLs were electrophoresed under reducing conditions and blotted with the indicated immune reagents. Isotypes produced by the cell lines are indicated below each lane. 

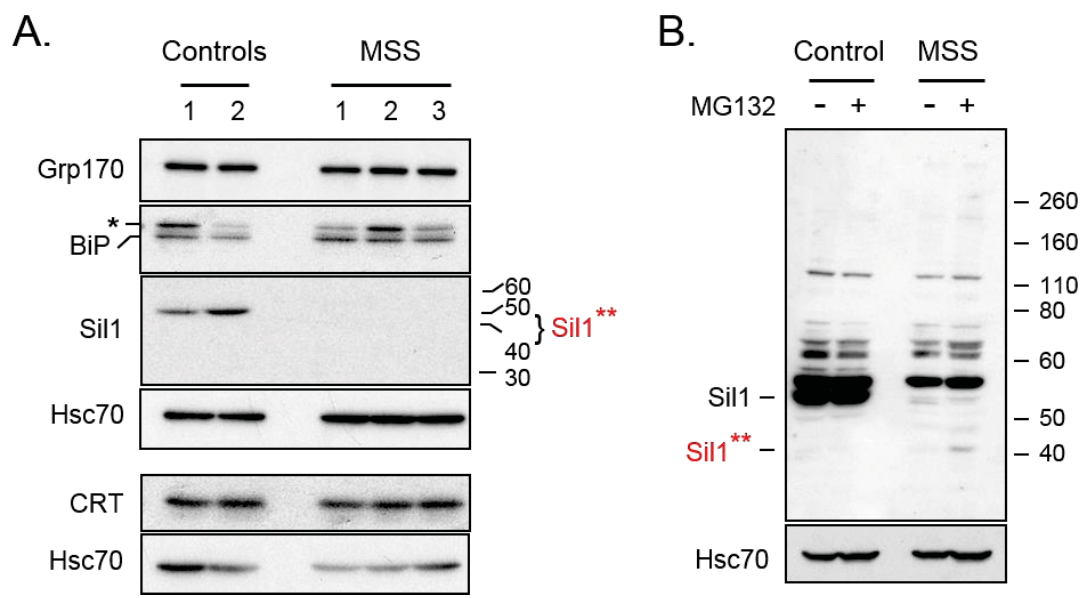

Figure 2-10. Disruption of Sil1 gene in MSS-patient-derived LBLs does not adversely affect the levels of ER chaperones.

(A) Direct cell lysates were prepared for western blotting and probed with the indicated antibodies to ER chaperones. Our rabbit polyclonal antiserum was used to detect SIL1 protein here, but similar results were obtained with the antibody from LifeSpan

Biosciences. The anti-human BiP reagent recognizes a non-specific band that is denoted with a single black asterisk. The expected mobility of the SIL1 truncated protein generated in the MSS cell lines (Sil1 ${ }^{* *}$ ) is indicated. (B) A control and MMS lymphoblastoid line were treated with MG132 (+) for 5 hours or left untreated (-). Lysates were prepared for western blotting and directly analyzed with an anti- SIL1 antibody from Proteintech. A band migrating with the predicted molecular weight for this mutant SIL1 protein (Sil1 ${ }^{* *}$ ) can be detected in the MSS line after proteasomal inhibition. 
A.

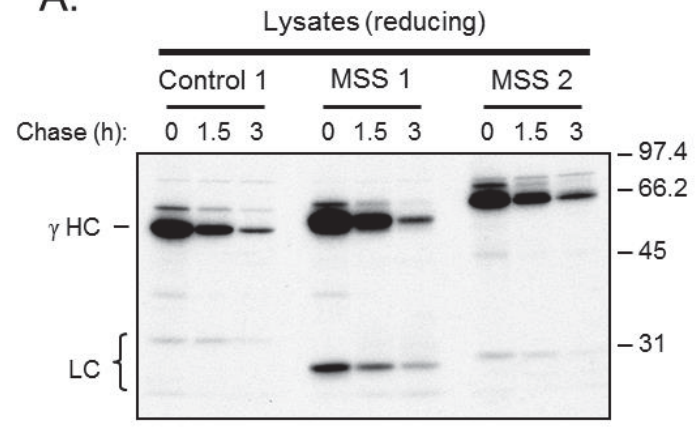

B.

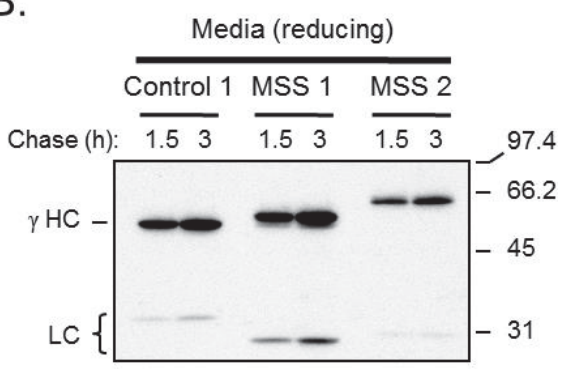

C.

Cysates (non-reducing)

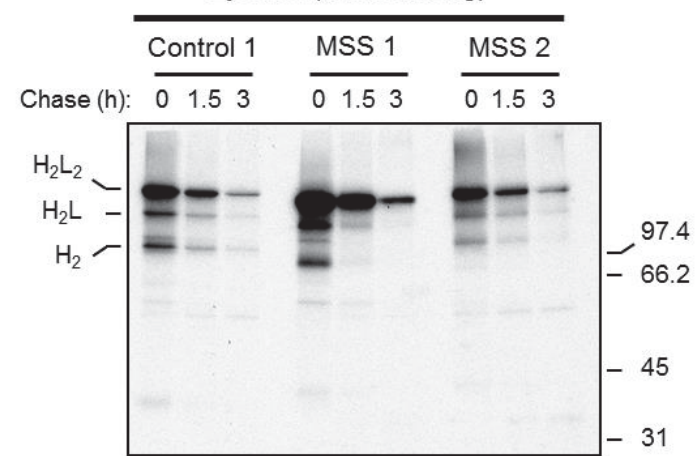

Figure 2-11. B Lymphoblastoid cell lines produced from individuals with Marinesco-Sjögren syndrome synthesize and secrete IgG antibodies normally.

One control and two LBL lines from MSS individuals that produce IgG were pulse labeled with ${ }^{35} \mathrm{~S}$ methionine and cysteine and chased for the indicated times. Cell lysates were immunoprecipitated with anti- $\gamma$ heavy chain and electrophoresed under reducing (A) or non-reducing (C) conditions. Culture supernatants were similarly immunoprecipitated and analyzed under reducing conditions (B). 
found no evidence that B cell development was affected nor was there a decrease in either the kinetics or magnitude of antibodies produced in response to antigen stimulation in vivo or LPS stimulation of purified splenic B cells for any isotype analyzed. This was not due to the fact that plasma cells do not make SIL1 protein, as we observed increases in this NEF during the course of differentiation in wild-type plasmablasts and lymphoblastoid lines from control individuals had readily detectable amounts of SIL1. We did not detect any unique changes in the complement of ER chaperones induced during plasma cell differentiation or any evidence that another chaperone system was utilized to support antibody synthesis in the absence of SIL1. Contrary to our findings one recent report on an individual with MSS noted decreased levels of serum IgG2 and $\operatorname{IgA}$, but not IgM (118). However, based on the fact that recurrent infections are not included in common symptoms experienced by MSS patients (78) and our data on normal Ig production in LBL cell lines from affected individuals, it seems more likely that the reduced levels of Ig in this patient were unrelated to the loss of SIL1 function.

\section{How is BiP released from Ig subunits to allow their assembly when SIL1 is absent?}

An obvious candidate for releasing BiP is Grp170, the resident ER large Hsp70 family member, which also possess nucleotide exchange activity (50). A recent study in yeast on the cytosolic orthologues of Grp170 and SIL1, Sse1 and Fes1 respectively, found that Sse1 was more critical for both protein folding and degradation, whereas neither protein was required for renaturation of heat-aggregated proteins (119). However, we did not detect an increase in Grp170 expression in the woozy splenic B cells above that which normally occurs during plasma cell differentiation, which is in contrast to the findings with Purkinje cells (91) and muscle tissue (100) from woozy mice. It is possible that sufficient levels of Grp170 are already present in the plasmablasts to induce the release of $\mathrm{BiP}$ from assembling antibodies. There are currently no data to establish relative levels of Grp170 versus SIL1 in a variety of tissues, to assess whether the loss of SIL1 might be felt more acutely in some tissues than others. A previous analysis of tissue blots from our lab found that SIL1 transcript levels were particularly high in placenta, liver, kidney, skeletal muscle, and heart (63). With the exception of a progressive myopathy, these represent tissues that are not usually affected in MSS patients $(78,99)$. A closer examination of relative levels of SIL1 and Grp170 in tissues that are affected by SIL1 loss compared to those that are not might shed light on this possibility. Alternatively, it is conceivable that additional BiP nucleotide exchange factors exist in the ER that have not yet been identified, which could compliment the loss of SIL1 in plasma cells and other non-affected tissue. The eukaryotic cytosol expresses a third family of NEFs, the Bag1 proteins (120), but there are no obvious ER orthologues in the mammalian databases. Lastly, we have considered the possibility that the association of LC is sufficient to by-pass the need for BiP or to displace wild-type BiP without requiring nucleotide exchange. In the case of $\operatorname{IgM}$ and $\operatorname{IgG} 2 \mathrm{~b}$, where $\mathrm{LC}$ can bind the $\mathrm{HC}$ co-translationally driving assembly through the $\mathrm{H} \rightarrow \mathrm{HL} \rightarrow \mathrm{H}_{2} \mathrm{~L}_{2}$ pathway, it is conceivable that the requirement for $\mathrm{BiP}$, and thus for a nucleotide exchange factor is relatively small. However, we also found that both mouse and human IgG subclasses that combine through the $\mathrm{H} \rightarrow \mathrm{H}_{2} \rightarrow \mathrm{H}_{2} \mathrm{~L} \rightarrow \mathrm{H}_{2} \mathrm{~L}_{2}$, which interact with BiP throughout the 
assembly process, were equally unaffected, making this possibility less viable but as yet untested in vitro.

\section{Why do SIL1 disrupted mice and MSS patients have defects that only appear to affect some systems?}

The dependence of only some tissues on SIL1 remains one of the puzzling manifestations of the disease and of the phenotypes observed in woozy mice. In fact, it appears that highly secretory organs like the pancreas, liver, and plasma cells, where one might expect adverse consequences when a component of the chaperone machinery is lost, are relatively less affected by SIL1 loss. Instead the effects are greatest in the Purkinje cells of the cerebellum, the eye lens, and skeletal muscle (78), which are not associated with the production of large amounts of secretory proteins that are BiP substrates. Perhaps the more classical secretory tissues are better able to respond to or accommodate changes in the client load of the ER. It is conceivable that there is some BiP:client specificity for the two NEFs of the ER, but this has yet to be demonstrated in any organism, organelle, or system.

Another interesting possibility is that the various clinical symptoms associated with MSS represent a progressive inability to maintain ER homeostasis with aging, which might occur at distinct times in various tissues, a phenomenon referred to as proteostasis collapse (121). In support of this possibility, the Purkinje cell loss leading to ataxia and the progressive myopathy associated with MSS and woozy mice can occur with quite different kinetics. While we have not rigorously examined immune responses in aged Sill ${ }^{\mathrm{Gt}}$ mice, we quantified splenic populations in a 22-month-old mouse and found no significant differences in any subpopulations (data not shown). However, one of our aged Sill ${ }^{\mathrm{Gt}}$ mice experienced a severe infection with Pasteurella pneumotropica, which usually only occurs in immunodeficient mice (122). As sufficient numbers of our Sill ${ }^{\mathrm{Gt}}$ mice age, we intend to revisit the ability of older mice to produce antibodies.

In summary, we have conducted a rigorous study of the effects of SIL1 loss on the synthesis of antibodies, the best characterized BiP-dependent substrate. We found no change in either the magnitude or kinetics of antigen or LPS-stimulated production of antibodies in the Sill $^{\mathrm{Gt}}$ mice compared to age-matched littermates. Furthermore, there was no evidence that the other ER NEF, Grp170 was abnormally up-regulated or that a different chaperone system was used to synthesize antibodies. Similar data were obtained with lymphoblastoid cell lines produced from individuals with MSS. We conclude that although loss of SIL1 in humans and mice can cause serious multi-system defects, these are not extended to the production of antibodies. 


\section{Materials and Methods}

\section{Generation of wild-type and Sill-disrupted mice}

The Sill gene-trap mouse strain B6;129Sil1 ${ }^{\text {Gt (pGT2TMpfa)1Slac }}$ was kindly provided by Dr. Susan Ackerman (87). Sill ${ }^{\mathrm{Gt}}$ heterozygous mice were crossed to generate homozygous woozy mice and wild-type littermates. At 3 weeks of age, DNA was isolated from mouse tail snips and genotyped as reported (87). The primers for the wildtype allele were: 5'-CTATAGCCCGCCTCAGGAGTGTTGTA-3' and 5'-

ATTGCCCCATAGAGCCATTTGTTGT-3', and those for the disrupted allele were: 5'CACCGGATGCAGAAAAGCCACAAT-3' and 5'-

GCAACTCGCCGCACATCTGAACTT-3'. Mutant mice were born at expected Mendelian ratios. The breeding and housing of mice were approved by the Animal Use and Care committee and Animal Research Center at St. Jude Children's Research Hospital, which adhere to NIH guidelines.

\section{Detection of mutant transcripts and protein in woozy mice}

To determine the relative transcript levels of SIL1 in Sill ${ }^{\mathrm{Gt}}$ mice compared to wild-type littermates, we isolated total RNA from wild-type and mutant plasmablasts after 4 days of LPS stimulation using the RNeasy ${ }^{\circledR}$ Plus Mini Kit (Qiagen) according to manufacturer's instructions. RT-PCR was performed with $2 \mu \mathrm{g}$ of total RNA using the High Capacity cDNA Reverse Transcription Kit (Applied Biosystems ${ }^{\circledR}$ ) and a PTC-200 Thermal Cycler (MJ Research). Quantitative Real Time PCR (qPCR) primers were obtained from Invitrogen. The primer pairs used were Sill FP1 5'AGGAATTTGATGAGCTGAACGT-3' and Sill RP1: 5'TCCTGTGCGTTATCCATCTGA-3', which produces a fragment that extends across exon 6 and the exon 6-exon 7 boundary, and detects both Sill ${ }^{\mathrm{WT}}$ and Sill ${ }^{\mathrm{Gt}}$ transcripts; Sill FP2: 5'-CTGCGCCACTTCCCCTAT-3' and Sill RP2: 5'-

GCAAACATCTTCTCAGTGACCA-3', which produces a fragment that extends across exon 9 and the exon 9-exon 10 boundary, and detects only Sill ${ }^{\text {WT }}$ transcripts; and HPRT FP: 5'-AGGGAGAGCGTTGGGCTTACCT-3' and HPRT RP: 5'CCTGGTTCATCATCGCTAATCACGA-3'. The qPCR was performed with 7900HT

Fast Real-Time PCR System (Applied Biosystems) using the Power SYBR ${ }^{\circledR}$ Green PCR Master Mix (Applied Biosystems). All qPCR reactions were carried out in triplicate. The signal obtained for SIL1 mRNA with both primer pairs was normalized to that obtained for HPRT mRNA, which served as an internal control. The value for wild-type SIL1 mRNA was set to 1 and the value for Sill ${ }^{\text {Gt }}$ mRNA presented as a fraction of this number. 


\section{Characterization of immune cell populations in spleen and thymus}

Murine spleen and thymus were excised from three, 3 to 6-month-old male C57BL/6 Sill ${ }^{\text {WT }}$ and Sill ${ }^{\text {Gt }}$ mice. Splenic cell suspensions were stained with CD3-APC, CD4-FITC, CD8-APC-Cy7, NK1.1-PE, B220-e605, Gr1-PE-Cy7, Mac1-Alexa700 and DAPI. Splenic subpopulations were analyzed as follows: $\mathrm{CD}^{+} / \mathrm{CD}^{+}, \mathrm{CD}^{+} / \mathrm{CD}^{+}$, $\mathrm{B} 220^{+}, \mathrm{Mac}^{+}, \mathrm{Gr}^{+}, \mathrm{Mac1}^{+} / \mathrm{Grl}^{+}$and NK1.1 ${ }^{+}$cells. Thymic cell suspensions were stained with Lin (B220/Mac1/Gr1)-FITC, CD3-PE, CD4-PerCP-Cy5.5, CD44-PE-Cy7, CD25-APC, CD8-Alexa700 and DAPI. T-cell subpopulations were identified as follows: double negative, DN (CD4 $\left./ \mathrm{CD}^{-}\right)$, DN1 (CD4 $\left./ \mathrm{CD}^{-} / \mathrm{CD} 25^{-} / \mathrm{CD}_{4} 4^{+}\right)$, DN2 (CD4 $/ \mathrm{CD}^{-}$ $/ \mathrm{CD} 25^{+} / \mathrm{CD}_{4} 4^{+}$), DN3 (CD4 $\left./ \mathrm{CD} 8^{-} / \mathrm{CD} 25^{+} / \mathrm{CD} 44^{-}\right)$, DN4 (CD4 $\left./ \mathrm{CD} 8^{-} / \mathrm{CD} 25^{-} / \mathrm{CD}^{-} 4^{-}\right), \mathrm{DP}$ $\left(\mathrm{CD}^{+} / \mathrm{CD}^{+}\right)$, CD4SP $\left(\mathrm{CD}^{+} / \mathrm{CD}^{-}\right)$and $\mathrm{CD} 8 \mathrm{SP}\left(\mathrm{CD} 4^{-} / \mathrm{CD}^{+}\right)$. Staining and flow cytometry were performed by the SJCRH Flow Cytometry and Cell Sorting shared resource on a LSR II Flow Cytometer (BD Biosciences) and analyzed using BD FACSDiva software (BD Biosciences). All antibodies were procured from BD Biosciences.

\section{Immunization protocol and ELISA for antigen-specific response}

Ten each of Sill ${ }^{\mathrm{WT}}$ and Sil1 ${ }^{\mathrm{Gt}}$ mice were immunized with a 1:1 homogenate of the T-dependent antigen 4-Hydroxy-3-nitrophenylacetyl conjugated to Keyhole Limpet Hemocyanin (NP15-KLH) (Biosearch Technologies) in Freund's complete adjuvant, injected intraperitoneally, and boosted after two weeks with NP ${ }_{15}-\mathrm{KLH}$ in Freund's incomplete adjuvant. Blood was collected on the indicated days by alternating retroorbital and tail vein bleedings. Antigen-specific serum IgM, IgG1, and IgG2b antibody titers were determined by direct ELISA. Bound antibody was detected with goat antimouse isotype specific, biotin-conjugated antibodies (Southern Biotech) and Streptavidin-conjugated alkaline phosphatase (Jackson ImmunoResearch). Absorbances at $410 \mathrm{~nm}$ were read on a Benchmark Plus plate reader after incubation with phosphatase substrate (Thermo Scientific). Serum concentrations were determined from standard curves with purified mouse IgM, IgG1 or IgG2b proteins (BD Pharminigen). Only triplicates with a $\mathrm{CV}<10 \%$ were used to calculate serum concentrations.

\section{Ex vivo stimulation of purified splenocytes with LPS}

Spleens were harvested from three 10 to 12 -week-old male Sil1 ${ }^{\text {WT }}$ and three age matched male Sill ${ }^{\mathrm{Gt}}$ mice, and single cell suspensions were prepared. After lysing red blood cells, the non-B cells were immune-depleted with antibodies to cell surface markers TER-119, CD4, CD8, Gr1, Mac1, and NK1.1 using an AutoMACS cell separator (Miltenyi Biotec). Aliquots of the resulting samples were stained with anti-B220 conjugated to Allophycocyanin (APC) and analyzed on a LSR II Flow Cytometer using BD FACSDiva software to determine the purity of remaining B cells, which was $>80 \%$ for all experiments. All reagents utilized were procured from BD Biosciences. The purified primary $\mathrm{B}$ cells were seeded at an initial cell density of $1 \times 10^{6}$ cells $/ \mathrm{mL}$ in RPMI 
1640 supplemented with 20\% (v/v) FBS Premium (Atlanta Biologicals), 2mM LGlutamine, 100U/mL Pen/Strep antibiotics, 1x (v/v) MEM Non-Essential Amino Acids (Gibco) and $55 \mu \mathrm{M}$ 2-Mercaptoethanol (Gibco) at $8 \% \mathrm{CO}_{2}$ and induced to differentiate

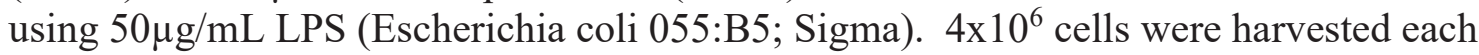
day along with an equivalent volume of culture supernatant. Media was changed daily and cells were maintained below a density of $2 \times 10^{6}$ cells $/ \mathrm{mL}$.

\section{EBV-transformed lymphoblastoid cell lines}

EBV-transformed peripheral blood lymphocytes from three related individuals with MSS and two unaffected family members were kindly provided by Dr. William R Wilcox (Emory University). The resulting cell lines were cultured in RPMI 1640 (Cellgro) supplemented with 10\% (v/v) FBS Premium (Atlanta Biologicals), $2 \mathrm{mM} \mathrm{L-}$ Glutamine, $100 \mathrm{U} / \mathrm{mL}$ Pen/Strep antibiotics and Gentamicin Reagent Solution $(10 \mu \mathrm{g} / \mathrm{mL})$ at $37^{\circ} \mathrm{C}$ in $5 \% \mathrm{CO}_{2}$.

\section{Western blotting}

Nonidet P-40 cell lysates from B lymphoblastoid lines and LPS-stimulated plasmablasts were produced as previously described (44). The resulting clarified lysates were designated as the NP-40 soluble fraction. The NP-40 insoluble pellets were dissolved in 50 $\mu \mathrm{l}$ Tris-SDS-buffer and prepared as described (123). For direct western blotting, the NP-40 soluble and insoluble fractions were normalized for equivalent cell number (plasmablasts) or protein amount (lymphoblastoid cells), and electrophoresed on $10 \%$ SDS-polyacrylamide gels under reducing or non-reducing conditions as indicated. In cases where IgM secretion was analyzed, a corresponding amount of culture supernatant was first immunoprecipitated with goat-anti-mouse $\mu$ and Captiv $^{\mathrm{TM}}$ PriMAB Protein A agarose beads. For experiments to determine if the mutant SIL1 protein made in LBLs produced from individuals with MSS was unstable, cells were treated with $10 \mu \mathrm{M} \mathrm{MG132}$ (Calbiochem) for 5 hours or left untreated. In all cases, after electrophoresis on SDS-polyacrylamide gels the separated proteins were transferred to Immun-Blot ${ }^{\mathbb{B}}$ PVDF membrane (Bio-Rad) and incubated with antibodies specific for the indicated proteins. Antisera to rodent BiP (42), SIL1 (63), ERdj3 (113), Grp170 (44), and Grp94 and calnexin (124) were produced in our lab. The anti-human BiP antiserum was kindly provided by Dr. Ineke Braakman (Utrecht University, Utrecht, The Netherlands) and the anti-ERdj1 antiserum was a generous gift from Dr. Richard Zimmermann (Universität des Saarlandes, Homburg, Germany). Antibodies to human Ig $\kappa \mathrm{LC}$, calreticulin, Hsc70, and actin were purchased from Santa Cruz, and the anti-ERdj5 was obtained from Abnova. Two anti- SIL1 reagents were purchased; one from LifeSpan Biosciences, which was raised to a fragment that is preserved in the MSS mutant SIL1 proteins studied here, and the other from Proteintech, which was produced against fulllength human SIL1 protein. Goat anti-mouse $\mu \mathrm{HC}$ and $\kappa \mathrm{LC}$, anti-human $\mu \mathrm{HC}, \gamma \mathrm{HC}$, and $\lambda$ LC antibodies were obtained from Southern Biotech, and horseradish peroxidase- 
conjugated, species-specific secondary immune reagents were purchased from Santa Cruz Biotechnology.

\section{Metabolic labeling}

$4 \times 10^{6}$ plasmablasts from day 2 and 3 LPS cultures were pulse-labeled with $300 \mu \mathrm{Ci}$ EasyTag ${ }^{\mathrm{TM}}$ EXPRESS $^{35}$ S Protein Labeling Mix (Perkin Elmer) for 20 minutes and either lysed immediately ( $0 \mathrm{~h}$ chase) or re-cultured for $1 \mathrm{~h}$ in complete media supplemented with $2 \mathrm{mM}$ unlabeled L-Cys and L-Met (1h chase). In the case of anti-BiP immunoprecipitations, 10 units/ml of apyrase (Sigma-Aldrich) was added to the lysing buffer, whereas for anti-Grp170, the lysis buffer was supplemented with $2 \mathrm{mM} \mathrm{Mg}^{2+}$-ATP (Sigma-Aldrich) and 25mM KCl (Fisher Scientific) as described (44). Antisera to calreticulin (Abcam) and calnexin were added directly to unsupplemented cell lysates. In all cases immune complexes were isolated with Protein A agarose beads, and isolated proteins were subjected to reducing SDS-PAGE. The resulting gels were incubated in Amplify (GE Healthcare, Pittsburgh, PA), supplemented with 3\% glycerol, for $30 \mathrm{~min}$ at room temperature before they were dried, and exposed to film (Fuji Film). To determine if SIL1 deficient lymphoblastoid cells exhibit a difference in their ability to synthesize and secrete antibodies, we performed pulse-chase experiments. $6 \times 10^{6}$ lymphoblastoid cells were labeled with $400 \mu \mathrm{Ci}^{35} \mathrm{~S}$ methionine and cysteine for 30 minutes and chased in complete media for $0,1.5$ or 3 hours, respectively. Post-lysis, the NP-40 soluble fraction and an equivalent amount of culture supernatant were first immunoprecipitated for $\gamma \mathrm{HC}$ and then analyzed on reducing or non-reducing SDS polyacrylamide gels as indicated.

\section{Sill sequencing}

Sequence analysis was performed for the Sil1 gene (GenBank NM_001037633) and transcript from control and MSS-derived lymphoblastoid cells. Genomic DNA (gDNA) and RNA were extracted using the DNeasy ${ }^{\circledR}$ Blood \& Tissue Kit (Qiagen) and RNeasy ${ }^{\circledR}$ Plus Mini Kit (Qiagen), respectively, according to the manufacturer's instructions. Using gDNA as template, we PCR-amplified regions spanning exon 9 and the 5' region of intron 9 using primer pairs 5'-GGGACTAATTTGGGGCACCT-3' and 5'-CCTCAGCCTTTTGTCCCCTC-3'; and the 3'region of intron 9 and the translated region of exon 10 using primer pairs 5'-TGGGGATAGAGGAAGAGGTGG-3' and 5'GTCCTCCTGCCTGAGAAGC-3'. $2 \mu \mathrm{g}$ of total RNA was used to synthesize cDNA as described above. We PCR-amplified the region spanning exons 9-10 using primer pairs 5'-AAGAAGAAGGTCCTGTTTGCAC-3' and 5'-GAAGTAGCCCTCGTCCTCAC-3', to verify the frameshift mutation caused by c.1030-18G $>$ A $(86,115)$. Primer pairs were removed using the Wizard ${ }^{\circledR}$ SV Gel and PCR Clean-up System (Promega), and products were sequenced using Big Dye ${ }^{\circledR}$ Terminator (v3.1) Chemistry on Applied Biosystem 3730XL DNA Analyzers by the Hartwell Center, St. Jude Children's Research Hospital. 


\section{Statistical analysis}

Statistical analyses were computed using Student's $t$ test utilizing GraphPad Prism 6 (GraphPad Prism Software, Inc). Error bars represent means \pm s.d., except in Figure 2-3 where error bars indicate interquartile range of values. Statistical significance was concluded at $P<0.05$. 


\section{CHAPTER 3. SIL1, THE ENDOPLASMIC-RETICULUM-LOCALIZED BIP CO-CHAPERONE, PLAYS A CRITICAL ROLE IN MAINTAINING SKELETAL MUSCLE PROTEOSTASIS AND PHYSIOLOGY*}

\section{Introduction}

Nearly one-third of the human genome encodes proteins that enter the endoplasmic reticulum (ER) lumen, where they are often modified by N-linked glycans and fold into complex tertiary and quaternary structures that are stabilized by disulfide bonds (1). This intricate maturation process is vulnerable to mistakes at any of these steps, which could result in aberrant proteins that are devoid of function at best, and prone to toxic aggregate formation at worst. The ER is populated with a host of molecular chaperones that bind directly to vulnerable unfolded proteins and serve to aid in their proper maturation by restricting off-pathway folding intermediates.

Alternatively, if this process fails some of these same chaperones can identify and target the unfolded or misfolded clients for degradation through a process referred to as ERassociated degradation (ERAD) $(125,126)$. These stringent ER quality control (ERQC) measures ensure that only properly modified, folded, and assembled proteins are allowed to traffic to other organelles of the secretory pathway, to be expressed at the cell surface, or to be secreted $(92,127)$. Intrinsic to the ERQC machinery is a feed-back mechanism that allows the ER to respond to imbalances in normal protein folding by activating the Unfolded Protein Response (UPR) in an effort to adjust and maintain ER homeostasis (73).

ER chaperones can be grouped into two major families - the Hsp70 and the lectin chaperones - each having numerous co-factors that regulate their activity and interactions with nascent client proteins. Like all Hsp70 family members, the ER cognate BiP binds client proteins in an adenosine-nucleotide-regulated manner. The ATP-bound form of BiP readily engages clients, albeit with low affinity. This triggers ATP hydrolysis, transforming BiP into a high affinity, ADP-bound form (128). Two sets of co-factors further regulate BiP's ATPase cycle; ER-localized DnaJ-like proteins (ERDJs) and nucleotide exchange factors (NEFs). A subset of known ERDJs (ERDJ3-6) can directly bind clients, transfer them to BiP, and assist in promoting either pro-folding or prodegradation functions of $\mathrm{BiP}$ (55). Unfolded clients are protected from aggregation or misfolding while bound to BiP, but must be released in order to fold completely. This requires the action of NEFs that specifically release ADP from the BiP-client complex, allowing a new molecule of ATP to rebind. Consequently, the affinity of

* Reprinted from final submission with open access permission. Ichhaporia, V. P., Kim, J., Kavdia, K., Vogel, P., Horner, L., Frase, S., \& Hendershot, L. M. (2018). SIL1, the endoplasmic-reticulum-localized BiP co-chaperone, plays a crucial role in maintaining skeletal muscle proteostasis and physiology. Disease Models \& Mechanisms, 11(5), dmm033043. 
BiP for the bound client protein is lowered, leading to its release. Two ER localized proteins have been identified that possess NEF activity; SIL1 (63) and GRP170 (44). Both are ubiquitously expressed but do not appear to be completely redundant. While both SIL1 and GRP170 contribute to the release of cholera toxin from BiP, allowing it to be retrotranslocated to the cytosol (129); only GRP170 is able to stimulate the release of SV40 viral particles from BiP for their ER-to-cytosol transportation (130). Similarly, GRP170 has been reported to function in the release of two misfolded proteins from BiP (i.e., the NHK-variant of $\alpha 1$-antitrypsin and a transthyretin mutant), thereby promoting their degradation, whereas SIL1 does not appear to contribute in this case (131).

While unique functions for SIL1 in either BiP-mediated folding or degradation of endogenous clients have largely eluded identification, the discovery that mutations in SIL1 are the leading cause of Marinesco-Sjögren syndrome (MSS) indicates that there are SIL1-specific functions $(64,86)$. MSS is an autosomal recessive, multisystem disorder that adversely affects Purkinje cells, leading to a profound cerebellar ataxia, and is further clinically characterized by progressive myopathy and bilateral cataracts, among other tissue pathologies $(99,115)$. Mutations in GRP170 have not yet been linked to any disease, but attempts to make a GRP170-null mouse were not successful, revealing it to encode an essential protein (132). GRP170 is also a bona fide molecular chaperone (44, 110). This might account for the mouse embryonic lethality observed upon its loss, because SIL1 has no known chaperone function that could compensate. However, it remains unclear why the lack of SIL1 causes no apparent problem in some tissues (133) while others are severely crippled, since both proteins are highly expressed in most secretory tissues $(63,134)$.

Our goal was to identify the molecular pathway(s) underlying the progressive myopathy associated with MSS. Two mouse models have been created in which the Sill gene is disrupted in all tissues; one by a spontaneous transposon-mediated insertion known as the woozy mouse, Sill ${ }^{\mathrm{wz}}$, and the other by gene-trap methodology, referred to as the Sill $^{\mathrm{Gt}}$ mouse model (87). These mice have been reported to phenocopy at least some of the MSS-associated pathologies, including severe Purkinje cell loss leading to ataxia in both models (87), and myopathy in the Sill ${ }^{\mathrm{wz}}$ mouse model (100). Using the Sill ${ }^{\text {Gt }}$ mouse model, we observed a progressive loss of skeletal muscle mass and strength, which is characterized by a multifocal, myogenic, myopathy. Proteomic analyses revealed that the effects of SIL1 loss extended far beyond the ER, compromising all major organelles and pathways critical to normal muscle physiology. Consistent with a function for SIL1 in ERQC, we observed disruption of ER homeostasis, up-regulation of ER chaperones and components of cellular protein degradation pathways, and aberrant expression of secretory pathway proteins critical for maintaining glucose and protein homeostasis in skeletal muscles. Our analyses provide molecular insights into why disruption of a component of the ERQC machinery results in loss of skeletal muscle mass, strength, and function. 


\section{Results}

\section{Sil1 $^{\mathrm{Gt}}$ mice display evidence of a progressive myogenic myopathy}

As the Sill $^{\mathrm{Gt}}$ mice aged, we noticed that they began to have difficulty holding on to the wired food tray while eating. We hypothesized that this might be indicative of a muscular weakness, similar to that observed in MSS patients $(80,117)$, and in keeping with pathology and biochemical data obtained from Sill ${ }^{\mathrm{wz}}$ mice (100). We used an inverted screen test to examine limb muscle strength of Sil1 ${ }^{\mathrm{Gt}}$ mice and their wild-type littermates at ages ranging from 1-22 months (Figure 3-1A). As early as 6 months, Sil1 ${ }^{\mathrm{Gt}}$ mice displayed a significantly reduced ability to hold on to the inverted screen, which became progressively worse with age. Necropsy revealed a corresponding decrease in Sill ${ }^{\text {Gt }}$ quadriceps mass with age, which was not observed in soleus or heart (Figure 3-1B and Figure 3-2).

To characterize the loss in muscle strength and mass, we conducted histological and electron microscopy (EM) analyses on a variety of muscle groups. Hematoxylin and eosin (H\&E) staining showed that a subset of Sill ${ }^{\mathrm{Gt}}$ skeletal muscles had numerous fibers with internalized nuclei and myofibril disruption (Figure 3-3A, D), and displayed evidence of adipose infiltration (Figure 3-4C, D). Staining of skeletal muscles for laminin, a major component of the extracellular matrix, revealed muscular dystrophy with a loss of the typical polygonal myofiber morphology in Sil1 ${ }^{\mathrm{Gt}}$ skeletal muscles and a wide range in myofiber diameter, including large, rounded hypertrophic fibers and shrunken, degenerating atrophic fibers (Figure 3-4A, B). Immunostaining of skeletal muscle cross-sections for IgG $(150 \mathrm{kDa})$ and $\operatorname{IgM}(900 \mathrm{kDa})$ did not show accumulation of these serum proteins in Sill ${ }^{\mathrm{Gt}}$ skeletal muscles, indicating that the hypertrophic myofibers maintained intact sarcolemmal integrity (Figure 3-4E-H). Epaxial muscles are composed of both predominantly glycolytic fast myofibers and largely oxidative slow myofibers, which can be distinguished by the type of myosin heavy chain (MyHC) they express. Thus, MyHC immunostaining can be readily used to determine if there is any specificity in the type of myofibers that are affected upon SIL1 disruption. We found that the affected fibers were almost entirely limited to those expressing MHC type II, indicative of fast-twitch, glycolytic myofibers (Figure 3-3B, E). In keeping with this observation, the soleus, which is predominantly composed of oxidative myofibers, did not appear to be affected in any of the analyses performed, whereas the biceps brachii, gastrocnemius, quadriceps femoris, and tibialis anterior, which are all largely made up of glycolytic myofibers, showed significant pathological changes as observed in (Figure 3-3 and Figure 3-4). The heart, composed of specialized oxidative cardiomyocytes, also remained unaffected in these mice, arguing that glycolytic myofibers are uniquely vulnerable to SIL1 loss. Immunostaining for desmin, an intermediate filament protein, revealed significant disruption of myofiber architecture and accumulation of this protein in degenerating Sil1 ${ }^{\mathrm{Gt}}$ myofibers (Figure 3-3C, F). EM analyses were consistent with these findings, and additionally revealed dramatically dilated sarcoplasmic reticulartriads in atrophying fibers (Figure 3-3G, H), degenerating nuclei, rare mitochondrial abnormalities, and the double membranous structure enveloping the nucleus (Figure 

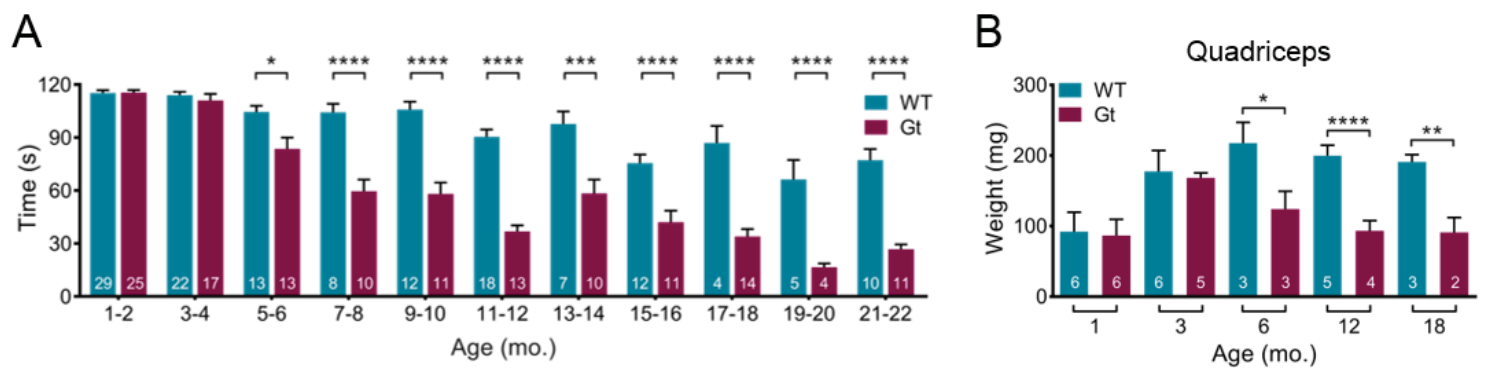

Figure 3-1. SIL1 is required to maintain muscle strength and mass as mice age.

(A) Age-matched wild-type and Sill ${ }^{\text {Gt }}$ mice ranging from 1 to 22 months were subjected to an inverted screen test for up to 120 seconds, and the duration of time they were able to hold onto the screen was measured. Number of mice in each group is indicated in white within bars. Error bars indicate means \pm s.e.m. (B) Quadriceps femoris were obtained from wild-type and Sill ${ }^{\mathrm{Gt}}$ mice at the indicated ages, and number of mice included in each group is shown within bars. Error bars indicate means \pm s.d. Statistical differences were computed using unpaired, two-tailed Mann-Whitney tests (A) or Student's $t$-tests (B), and are indicated as ${ }^{*} P \leq 0.05, * * P \leq 0.01, * * * P \leq 0.001$, and $* * * * P \leq 0.0001$. 

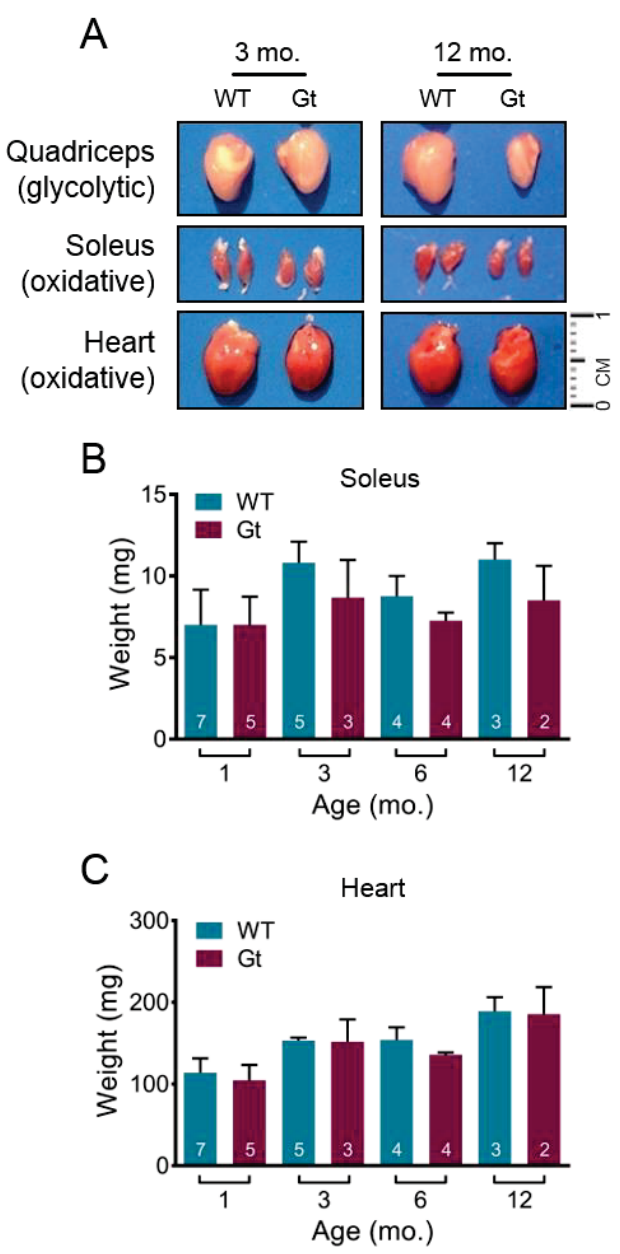

Figure 3-2. Loss of SIL1 predominantly affects glycolytic skeletal muscles.

(A) Representative images of quadriceps, soleus, and heart derived from wild-type and Sill $^{\mathrm{Gt}}$ mice at the indicated ages depicting that loss of SIL1 specifically affects the glycolytic quadriceps, while sparing the oxidative skeletal muscle, soleus, and specialized cardiac muscle. (B, C) Comparison of wild-type and Sil1 ${ }^{\mathrm{Gt}}$ soleus (B) and heart (C) weights at the indicated ages. Error bars indicate mean \pm s.d.; The number of mice is indicated within the bar graphs. Although there was a trend of slight decrease in the Sill ${ }^{\text {Gt }}$ soleus weights, these values were not statistically different from the wild-type soleus weights. 

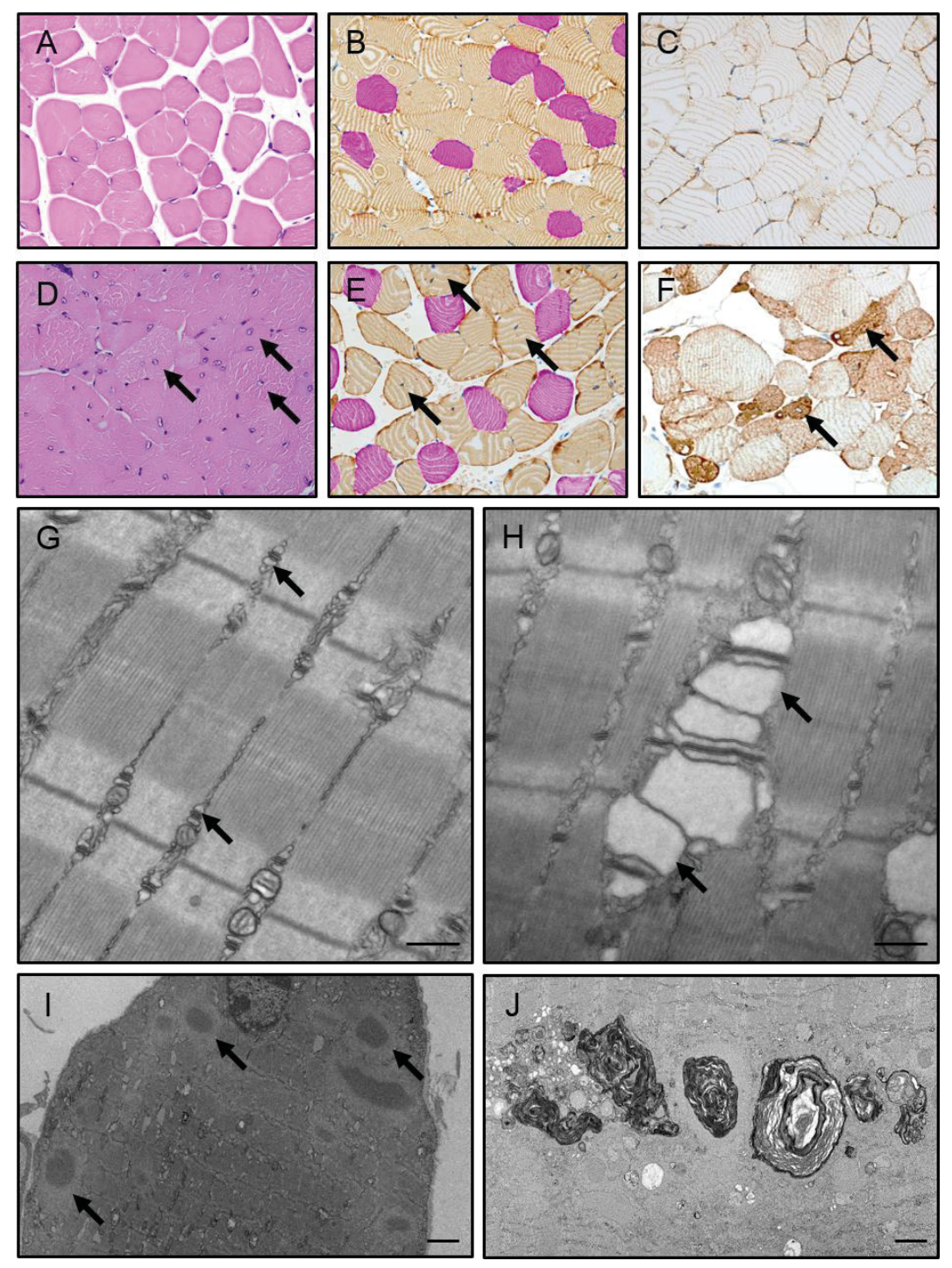

Figure 3-3. The myopathy resulting from SIL1 loss predominantly affects fast myofibers.

Skeletal muscle cross-sections shown here were derived from 12-mo. wild-type (A-C) and $\mathrm{Sill}^{\mathrm{Gt}}$ (D-F) mice. (A, D) H\&E staining revealed hypertrophic and atrophic fibers with internalized nuclei in the Sill ${ }^{\text {Gt }}$ skeletal muscles (arrows), compared to peripheral nuclei in Sill ${ }^{\mathrm{WT}}$ samples. (B, E) Epaxial muscles were immunostained with fast- (brown) and slow- (pink) MyHC antibodies. Arrows indicate myopathic myofibers with internalized nuclei, which stain positive for fast myofiber-specific MyHC. (C, F) Desmin staining shows evidence of aggregation and loss of cyto-architecture in degenerating Sil $1^{\mathrm{Gt}}$ skeletal muscle fibers (arrows). (G, H) Representative electron micrographs from 10 mo. wild-type $(\mathrm{G})$ and $\operatorname{Sill}^{\mathrm{Gt}}(\mathrm{H})$ bicep femoris displaying triads (arrows). (I, J) Electron micrographs from 10 mo.-old Sill ${ }^{\text {Gt }}$ quadriceps demonstrating accumulation of cytoplasmic inclusion bodies (I) and membranous whorls (J) (indicated by arrows), all of which are characteristic of MSS. (A-F) Images were captured at the same 40x magnification. Scale bars in (G, H) represent $400 \mathrm{~nm}$, and in (I, J) represent $2 \mu \mathrm{m}$. 
Figure 3-4. Sil1 ${ }^{\mathrm{Gt}}$ skeletal muscles display characteristic myopathic signatures.

(A-J) Representative hind-limb skeletal muscle cross-sections derived from 12 month-old wild-type (A,C,E,G,I) and Sil1 ${ }^{\mathrm{Gt}}(\mathrm{B}, \mathrm{D}, \mathrm{F}, \mathrm{H}, \mathrm{J})$ mice are stained with anti-laminin (A,B), an extracellular matrix protein; $\mathrm{H} \& \mathrm{E}(\mathrm{C}, \mathrm{D})$ depicting adipose infiltration in peripheral skeletal muscles, predominantly affecting muscles in close proximity of adipose tissue; anti-immunoglobulins $\operatorname{IgM}(\mathrm{E}, \mathrm{F})$ and $\operatorname{IgG}(\mathrm{G}, \mathrm{H})$ to asses sarcolemmal integrity. (I,J) H\&E staining of lumbo-sacral spinal cord sections display apparently normal motor neurons. Images were either captured at the same 40x (A-B,E-J) or 4x (C,D) magnifications, respectively. (K-M) Representative electron micrographs from 10 month-old wild-type (K) and Sill Gt (L,M) biceps brachii $(\mathrm{K}, \mathrm{L})$ and quadriceps $(\mathrm{M})$ reveal multiple myopathic features, including degenerating myonuclei (L; black arrows), electron-dense aggregates (L; white arrow), disruption of the cytoskeletal architecture, and a double membranous structure surrounding the nucleus (M; arrows), which is characteristic of MSS, and implicated to be a nuclear envelope pathology in woozy mice. Scale bars in $(\mathrm{K}, \mathrm{L})$ represent $2 \mu \mathrm{m}$, and in (M) $500 \mathrm{~nm}$. 


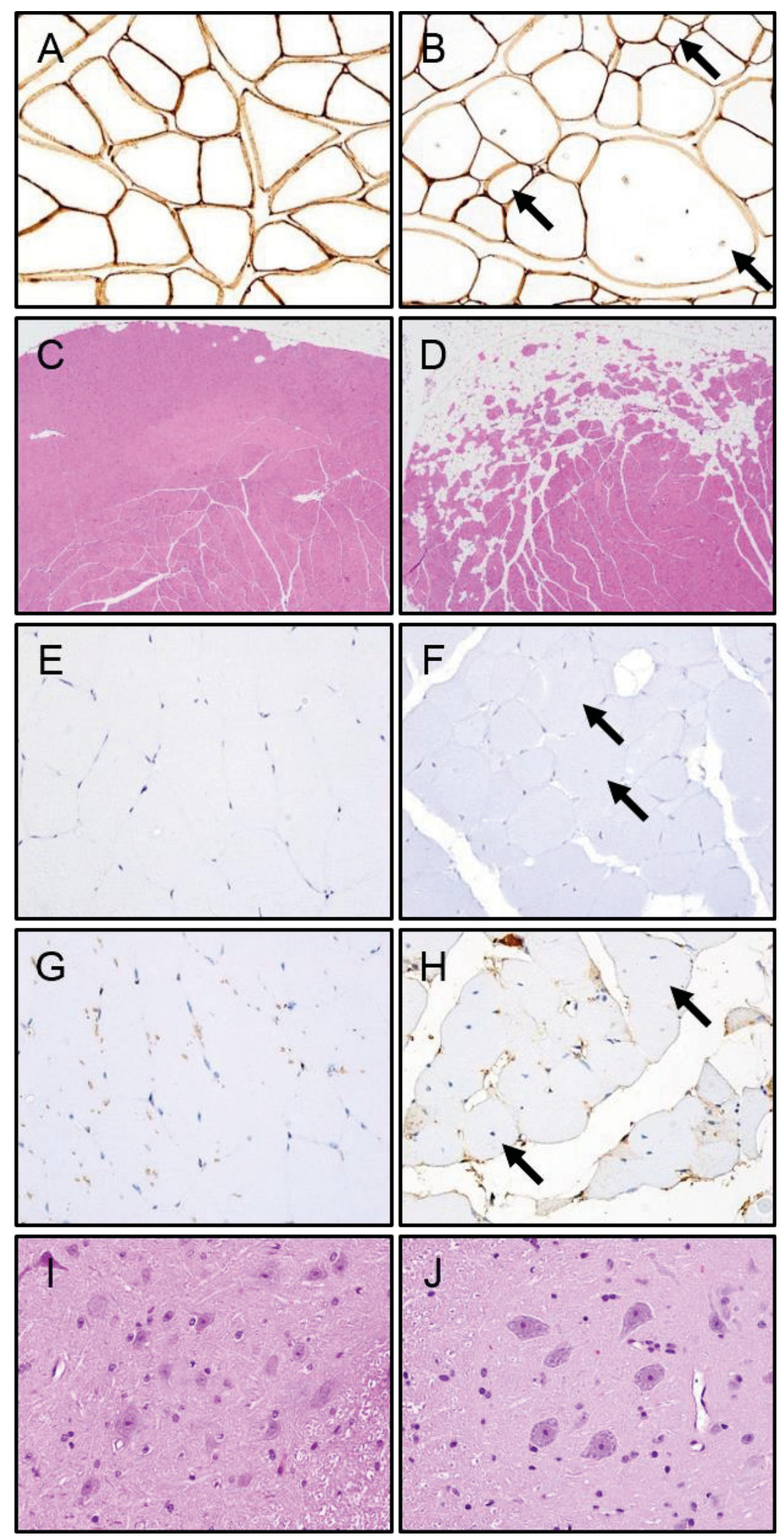



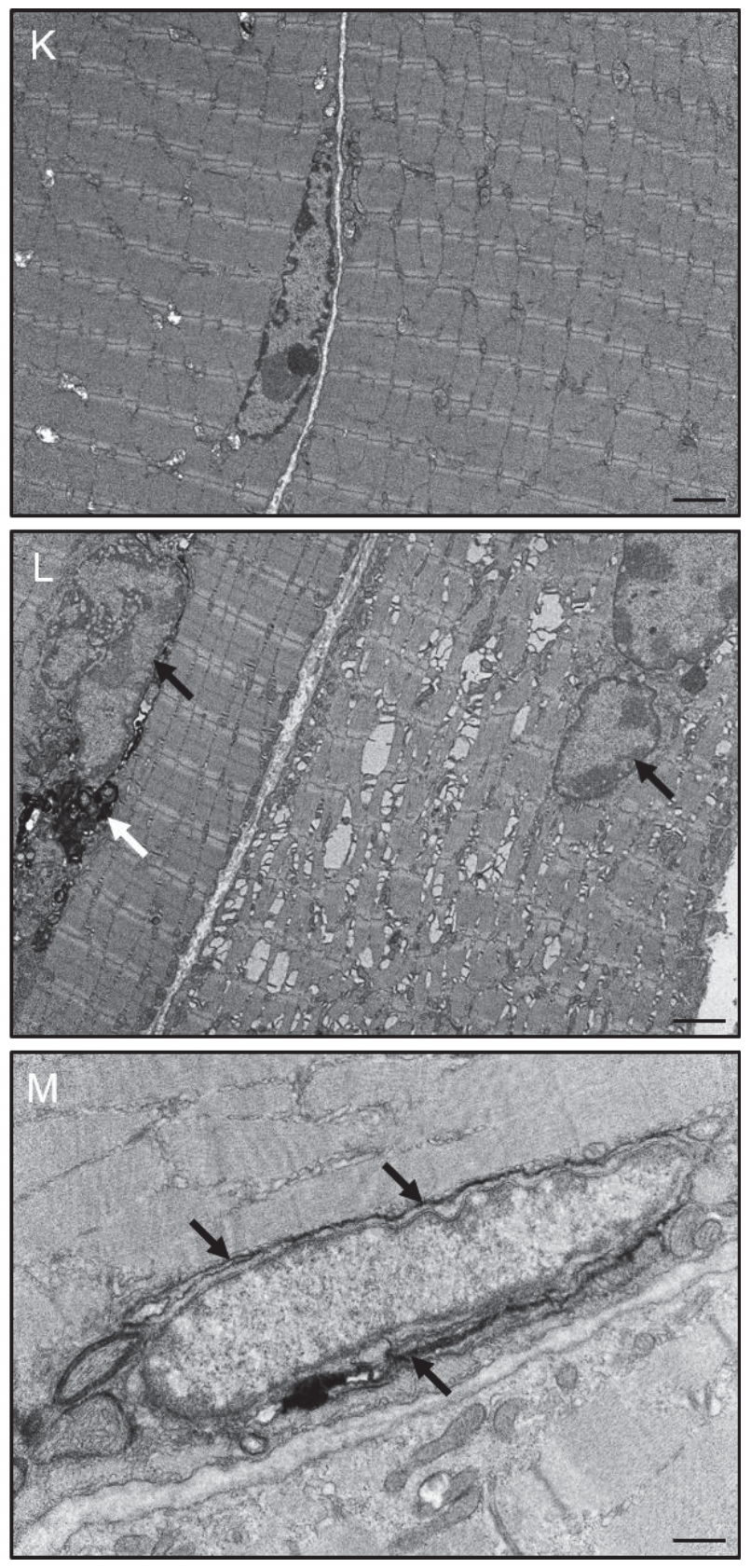

Figure 3-4. Continued. 
3-4K-M). We observed electron-dense material and cytoplasmic bodies in various myofibers, suggestive of protein aggregation, and an accumulation of membranous whorls, indicative of autophagosomes (Figure 3-3I, J). Together, these alterations strongly resemble ultrastructural changes observed in muscle biopsies of individuals with MSS $(80,81)$ and quadriceps from Sil ${ }^{\mathrm{wz}}$ mice $(100)$, and explain the decreased muscle mass and strength occurring in Sill ${ }^{\mathrm{Gt}}$ mice. The dispersed, multi-focal, and dystrophic myopathy we observed in Sill ${ }^{\text {Gt }}$ glycolytic skeletal muscles is most compatible with a myogenic myopathy. This is in contrast to the clustered myopathy with angular crosssectional myofiber morphology reported in Sill ${ }^{\mathrm{wz}}$ mice, which is more indicative of a neurogenic origin (135). Furthermore, H\&E-stained lumbo-sacral spinal sections did not reveal any obvious neurogenic pathology (Figure 3-4I, J), arguing against a major contribution of motor neuron defects in the Sill ${ }^{\mathrm{Gt}}$ progressive myopathy and revealing some differences in these two genetic models.

\section{SIL1 disruption leads to widespread changes in the quadriceps proteome}

In an unbiased approach to understand the Sil ${ }^{\mathrm{Gt}}$-associated myopathy at the molecular level, we performed proteomics analyses on quadriceps derived from wild-type and Sill ${ }^{\mathrm{Gt}}$ male mice at six months of age. This was the age at which the myopathy first became physiologically and pathologically evident. Among skeletal muscles strongly affected by SIL1 disruption, quadriceps femoris were our tissue of choice due to their composition of predominantly glycolytic myofibers, the availability of sufficient amounts of protein due to their relatively large mass, and the ease of dissection. Five mice of each genotype were sampled. A total of 5512 proteins were identified, of which 5384 could be quantified. Bioinformatics analyses identified 515 proteins whose expression was significantly altered $\left(\log _{2}\right.$ ratio $<-0.5$ or $\left.>0.5 ; P<0.01\right)$ in Sill $^{\text {Gt }}$ quadriceps, relative to those from wild-type mice. Of these, 301 proteins were up-regulated and 214 were decreased in expression (Supplementary Table S1). Manual curation of these proteins, sorted by their resident subcellular compartments according to the GeneCards database (http://www.genecards.org/) (136), revealed that loss of the ER co-chaperone SIL1 led to changes in protein expression extending to all major cellular compartments (Figure 3-5A). Gene-set enrichment analysis (GSEA) identified a significant number of proteins, belonging to signaling pathways and functions critical to muscle physiology, including insulin receptor (IR) signaling, glucose metabolism, and cation channel activity, to be altered in the quadriceps of 6-month-old Sill $1^{\mathrm{Gt}}$ mice. We verified the indicated changes in expression of a number of these proteins by western blotting (Figure 3-5B-E and Figure 3-6). Altered expression of ER chaperones, secretory pathway proteins that are synthesized in the ER, and elements of the cellular degradation machinery could be readily anticipated by loss of a component of the ER protein folding machinery. However, possible mechanisms leading to altered expression of proteins throughout other organelles was not immediately clear. 

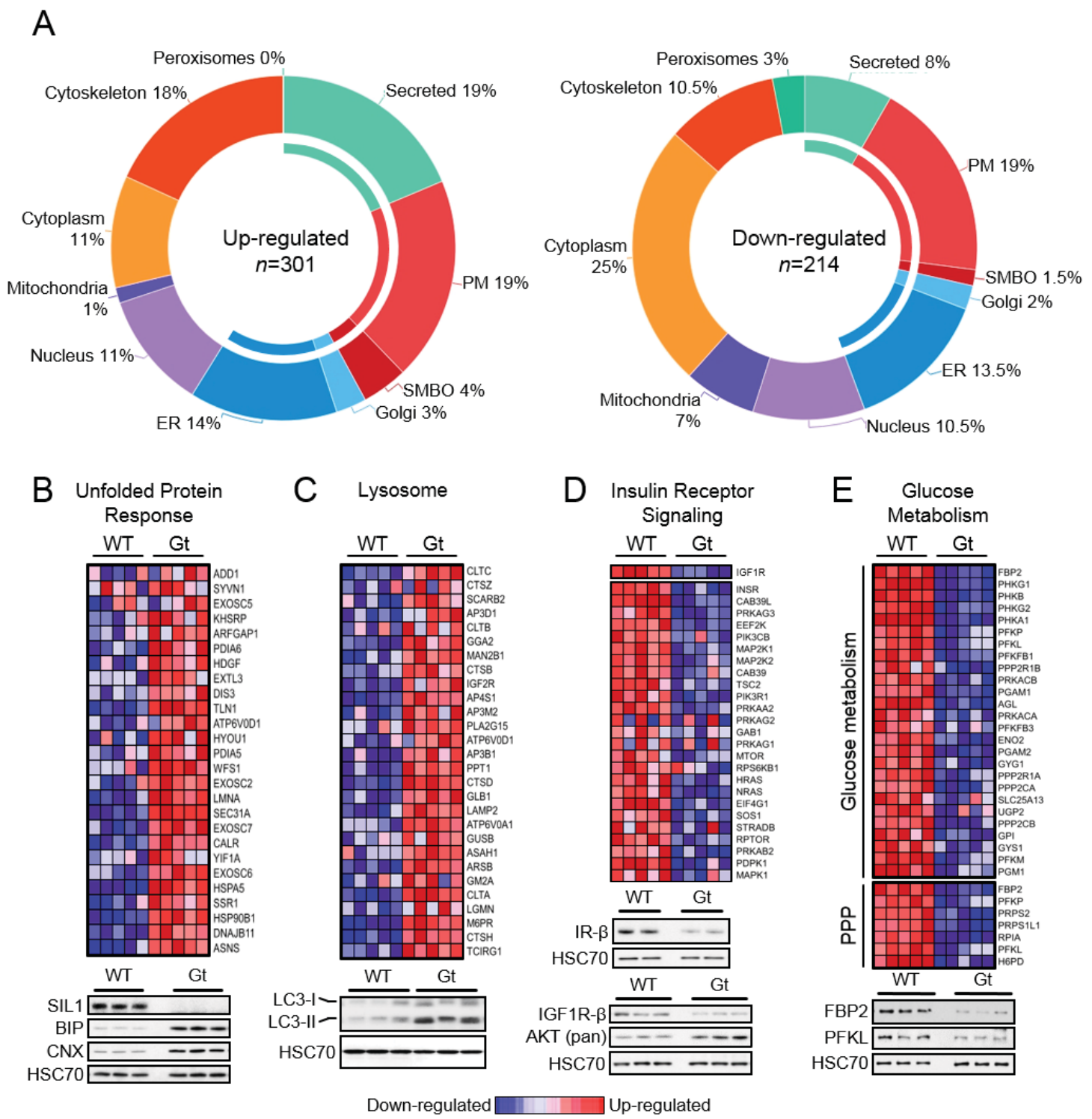

Figure 3-5. SIL1 disruption perturbs all major cellular organelles and pathways critical for normal quadriceps function.

(A) Graphical representation of the organellar distribution of proteins that were identified by proteomic analyses to be significantly $\left(\log _{2}\right.$ ratio $<-0.5$ or $\left.>0.5 ; P<0.01\right)$ upregulated (left) or downregulated (right) in quadriceps from 6-month-old $S i l 1^{\mathrm{Gt}}$ mice relative to that from wild-type mice $(n=5)$. Inner band indicates compartments of the secretory pathway. SMBO, single membrane bound organelles; PM, plasma membrane. (B-E) Heat maps of GSEA-identified gene-sets that are enriched $(\mathrm{B}, \mathrm{C})$ or depleted $(\mathrm{D}, \mathrm{E})$ in the $\mathrm{Sill}^{\mathrm{Gt}}$ quadriceps. PPP indicates Pentose Phosphate Pathway. Subsets of proteins for each gene-set were selected for verification by western blotting and are shown below the heat maps. 


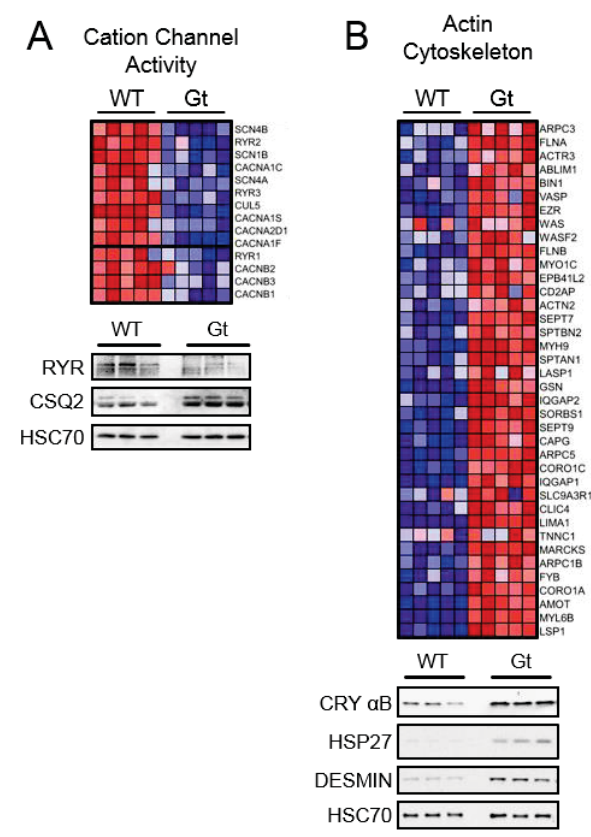

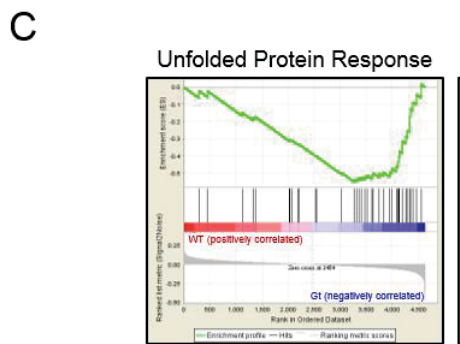

$\mathrm{NES}=-1.83 ; \mathrm{FDR}=0.036$

$P<0.0001$

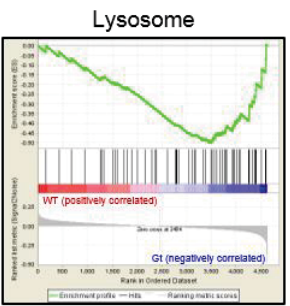

NES $=-1.83 ;$ FDR $=0.035$

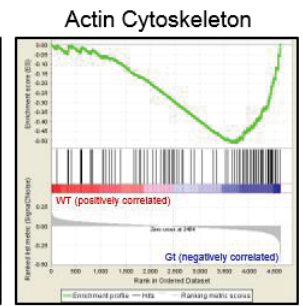

NES $=-2.03 ; \mathrm{FDR}=0.01$

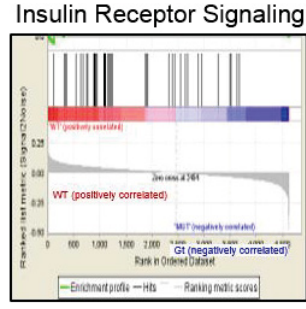

$\mathrm{NES}=2.09 ; \mathrm{FDR}=0.015$ $P<0.0001$

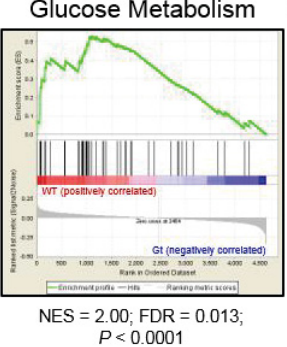

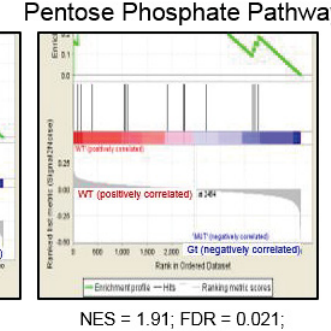

$P<0.0001$

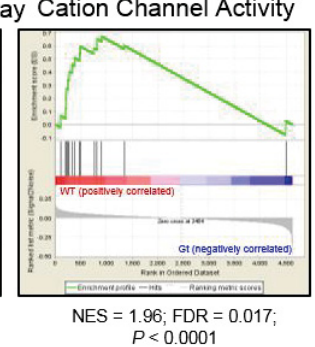

Figure 3-6. Proteomic study reveals that loss of SIL1 affects pathways critical for normal muscle physiology.

$(\mathrm{A}, \mathrm{B})$ Heat maps of $(\mathrm{A})$ proteins involved in cation channel activity that were significantly depleted, and (B) proteins annotated to play a role in the actin cytoskeleton organization that were significantly enriched in Sill ${ }^{\text {Gt }}$ quadriceps, respectively. A subset of proteins for each gene-set was selected for verification by western blotting and is shown below the heat maps. (A) Concomitant with a decrease in levels of the ryanodine receptor (RYR) $\mathrm{Ca}^{2+}$-release channel subunits, proteomics indicated a significant increase in the levels of calsequestrin 2 (CSQ2), which serves as the sarcoplasmic reticular $\mathrm{Ca}^{2+}$ buffering protein. (C) GSEA-derived enrichment plots for the respective pathways and associated heat maps depicted in Figure 3-5 and Figure 3-6A, B. 


\section{Loss of SIL1 disrupts ER homeostasis as mice age}

To begin to understand the widespread changes in protein expression that occurred upon SIL1 disruption, we examined time points both prior to and after the onset of muscular weakness, and began with proteins that contribute to ERQC, since this is where SIL1 functions. We combined quadriceps lysates from at least three male mice, for each age shown, to obtain an average expression for the proteins queried. The folding of secretory pathway proteins entering the ER as nascent chains is both aided and monitored by a host of molecular chaperones and their co-factors. Beginning as early as 3 months, we observed a slight increase in BiP expression in Sill ${ }^{\text {Gt }}$ quadriceps, which became more dramatic by 6 months and remained elevated (Figure 3-7A and Figure 3-8C). GRP170, the other ER NEF (50), showed a modest increase in expression beginning at 6 mo., whereas the lectin chaperones (Calnexin and Calreticulin) and their co-factor, ERP57, were more robustly up-regulated. The delayed up-regulation of these proteins suggested that loss of SIL1 suggested a progressive disruption of ER homeostasis. These proteins can be up-regulated at the transcriptional level by activation of the UPR due to the accumulation of unfolded proteins in the ER. We found evidence for the up-regulation of targets of all three upstream transducers (PERK, ATF6, and IRE1) in Sill ${ }^{\mathrm{Gt}}$ muscles relative to age-matched wild-type mice (Figure 3-8A-C). As an independent means of detecting disruptions in ER homeostasis, we examined the relative solubility of BiP and Calnexin. Both are normally soluble ER chaperones that bind unfolded proteins to help maintain them in a soluble form. However, if they are unable to do so, these chaperones can become trapped in detergent-insoluble complexes with client proteins. Unlike quadriceps, which were lysed in 8M Urea for proteomic and kinetics-ofonset studies, we used milder, NP40-mediated lysis conditions and examined NP40soluble and -insoluble fractions at three time points. As early as 3 months, a very small amount of BiP was detected in the NP40-insoluble fraction of Sill ${ }^{\text {Gt }}$ quadriceps, which became more prominent in the 6- and 12-months samples (Figure 3-7B). It is noteworthy that as the Sill ${ }^{\mathrm{WT}}$ mice aged, a small amount of BiP was also detected in the insoluble fraction. This is in agreement with reports of age-related disruptions in protein homeostasis, also referred to as proteostasis, which is a hallmark of skeletal muscle aging $(137,138)$.

Consistent with the observation of dilated sarcoplasmic reticular triads, the presence of BiP in the insoluble fraction implied that loss of SIL1 was likely to affect the maturation of cargo proteins that are synthesized in the ER and traffic through the secretory pathway. Indeed, the proteomics data indicated that secreted (19\% upregulated, $8 \%$ downregulated) and plasma membrane (19\% upregulated, 19\% downregulated) proteins represented a significant fraction of proteins with altered expression (Figure 3-5A). We chose to investigate four integral membrane proteins that showed reduced expression, including three subunits of heterodimeric cell surface receptors - insulin receptor $\beta$ chain (IR- $\beta$ ), insulin-like growth factor 1 receptor $\beta$ chain (IGF1R- $\beta$ ), and PDGF receptor $\alpha$ chain (PDGFR- $\alpha$ ) - as well as ryanodine receptors (RYR), which are transmembrane proteins that form calcium channels in the sarcoplasmic reticulum. Examination of quadriceps lysates at various time points confirmed a reduction in the expression of all four membrane proteins, although the kinetics and magnitude of these 
A

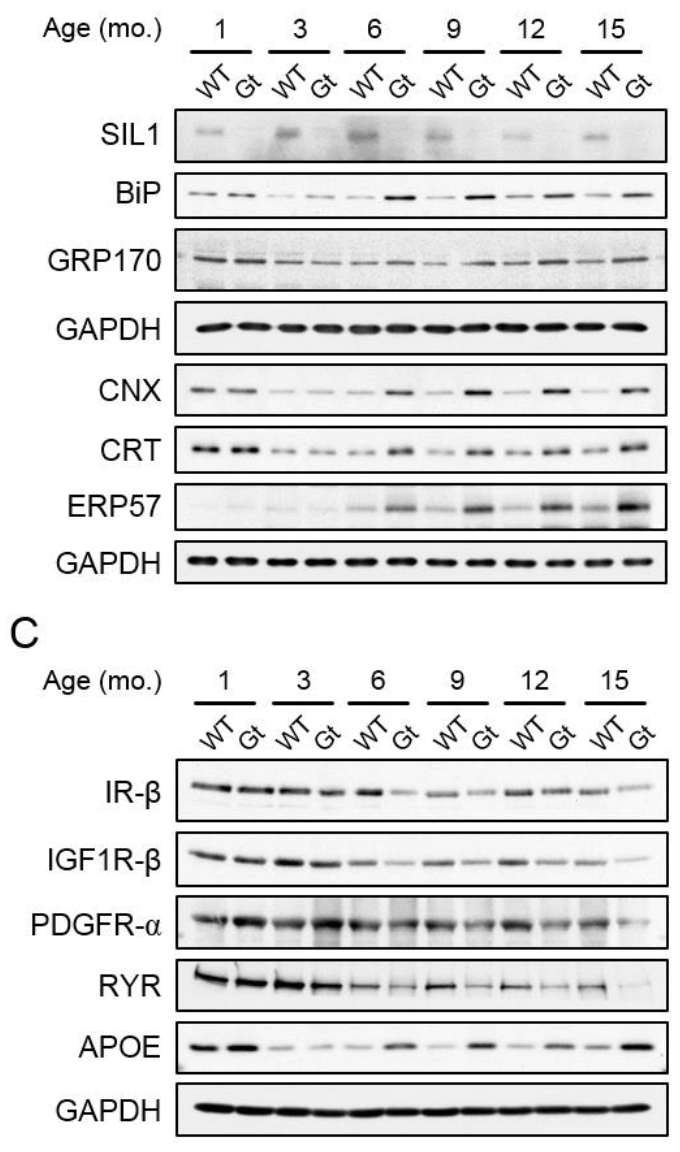

B

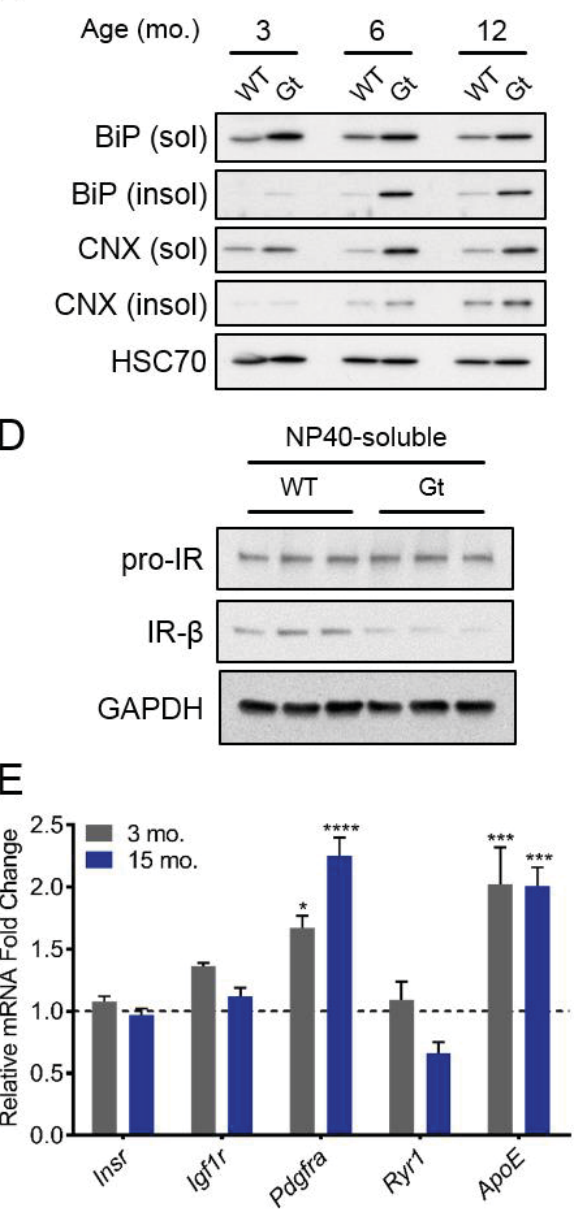

Figure 3-7. SIL1 is required to maintain ER proteostasis in quadriceps as mice age.

(A) Wild-type and Sil1 ${ }^{\mathrm{Gt}}$ quadriceps lysates ( $n=3-5$ pooled), solubilized in $8 \mathrm{M}$ urea, isolated from mice at the depicted ages were normalized for an equivalent amount of total protein, pooled, and subjected to reducing SDS electrophoresis, followed by transfer to PVDF membranes, and blotting with the indicated immune-reagents for ER chaperones and co-chaperones. GAPDH serves as a control for loading. (B) Wild-type and Sill ${ }^{\mathrm{Gt}}$ quadriceps obtained from mice at the designated ages were lysed with NP40 lysis buffer. The resulting NP40-soluble and NP40-insoluble fractions were processed for western blotting with BiP and calnexin antisera. HSC70 serves as a loading control for the NP40soluble fraction. (C) Urea-solubilized quadriceps lysates derived from mice of the indicated genotypes and ages were probed with antibodies to proteins that traffic through the secretory pathway. (D) NP40-solubilized quadriceps lysates from 12-month-old mice were processed for western blotting to detect the insulin receptor precursor (pro-IR) and the mature subunit (IR- $\beta$ ). (E) Graphical representation of normalized mRNA fold changes of proteins probed in (C) from Sill ${ }^{\text {Gt }}$ quadriceps, relative to wild-type quadriceps $(n=3)$ (set to 1; indicated by the dotted line), at 3 (gray) and 15 (blue) months. Error bars indicate means \pm s.e.m. Statistical differences were computed using unpaired, two-tailed Student's $t$-tests, and are indicated as $* P \leq 0.05$, $* * * P \leq 0.001$, and $* * * * P \leq 0.0001$. 


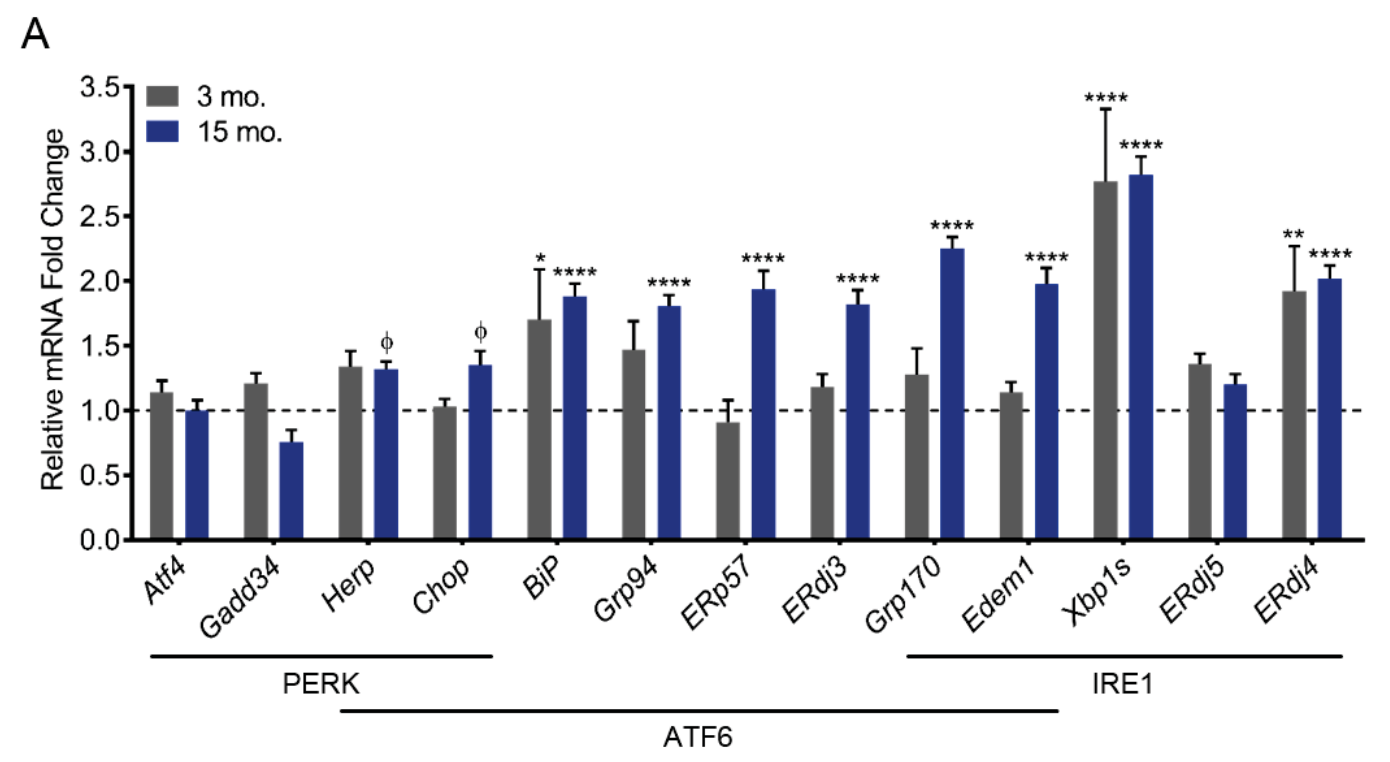

B

C
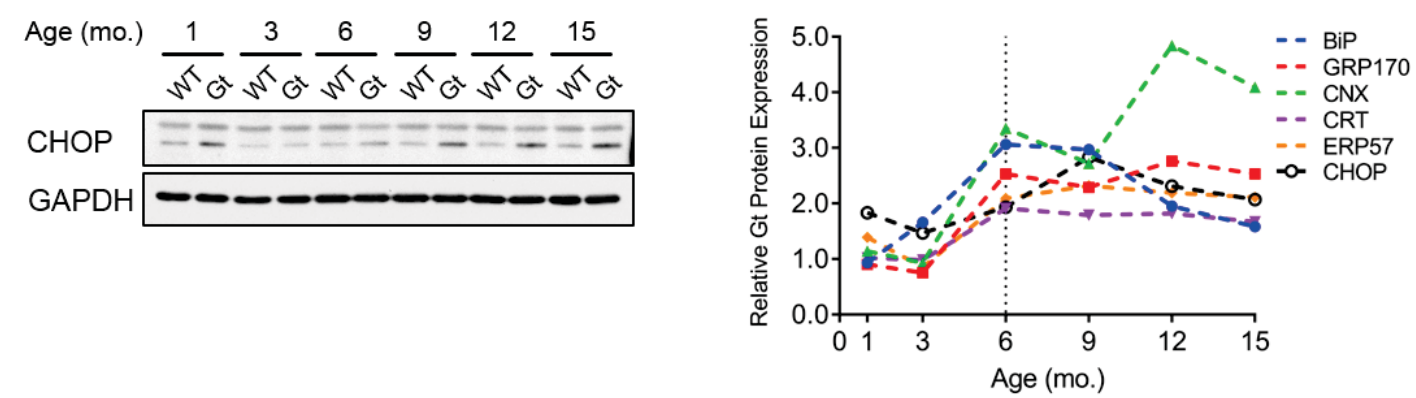

Figure 3-8. Loss of SIL1 leads to activation of the Unfolded Protein Response.

(A) Graphical representation of the indicated transcripts from Sill ${ }^{\text {Gt }}$ quadriceps, relative to the levels in wild-type quadriceps $(n=3)$, and represented as fold change at 3 (gray) and 15 (blue) months. The arm of the UPR that regulates these components during ER stress is indicated below the bar graph. Error bars indicate means \pm s.e.m. Statistical differences were computed using unpaired, two-tailed Student's $t$-tests, and are indicated as ${ }^{\phi} P<0.1$ but $>0.05,{ }^{*} P \leq 0.05,{ }^{*} P \leq 0.01, * * * P \leq 0.001$, and $* * * * P \leq 0.0001$. (B) Ureasolubilized lysates from Figure 3-7 were probed for CHOP. (C) Graph represent densitometric quantitation of western blotting data from Figure 3-7 and Figure 3-8B. Dotted vertical line at the 6-month-time point indicates the age of onset of muscular weakness in Sill ${ }^{\mathrm{Gt}}$ mice and at which quantitative proteomic profiling of quadriceps was carried out. 
changes varied slightly (Figure 3-7 and Figure 3-9A). We quantified their mRNA levels to ensure that these changes were not due to a transcriptional effect. IR- $\beta$ and IGF1R- $\beta$ transcripts were not significantly altered at either time point tested in Sil1 ${ }^{\mathrm{Gt}}$ mice, whereas PDGFR- $\alpha$ transcripts were actually increased compared to that in Sill ${ }^{\text {WT }}$ skeletal muscles

(Figure 3-7). In fact, when NP40-soluble protein lysates from 12-month quadriceps were analyzed, although the insulin receptor precursor levels were nearly identical to those in wild-type muscles, we observed a significant reduction in mature IR- $\beta$ subunit expression in Sill $^{\mathrm{Gt}}$ muscles (Figure 3-7D and Figure 3-9C). In the case of IGF1R, both the precursor and mature forms were decreased (Figure 3-9B, C). Thus, the reduced expression of these three proteins was likely a result of decreased protein stability due to disrupted ER homeostasis. However, Ryrl mRNA was modestly reduced making it more difficult to attribute the extent of change in protein levels to a folding defect. Conversely, our proteomic data indicated that the levels of multiple apolipoproteins (APOM, APOA1, APOA2, APOD, and APOE), which are secreted proteins (139), were increased in Sil1 ${ }^{\mathrm{Gt}}$ quadriceps. Indeed, western blotting for cellular APOE, which has been found to abnormally accumulate in Inclusion Body Myositis (140), showed a readily detectable increase as early as 6 months and remained elevated throughout the latter time points (Figure 3-7C and Figure 3-9A). While ApoE transcripts were up-regulated in Sill $^{\mathrm{Gt}}$ samples, they did not change between 3 and 15 months of age, whereas the protein levels increased significantly at 15 months compared to 3 months (Figure 3-7E), implying differences in post-translation regulation of this protein in Sill ${ }^{\mathrm{Gt}}$ myofibers. Importantly, unlike membrane proteins, which remain cell-associated when properly folded, secretory proteins exit the cell. Thus, a faster rate of transport for the properly folded APOE protein in the Sill ${ }^{\mathrm{WT}}$ muscle compared to the rate at which a misfolded protein is recognized and targeted for degradation could account for the increased APOE protein levels in Sill ${ }^{\mathrm{Gt}}$ skeletal muscles.

\section{Evidence for inadequate compensation by cellular degradation systems}

Based on the demonstration of altered expression of several secretory pathway proteins, and because $\mathrm{BiP}$ and the lectin chaperones participate in both the folding of clients and the targeting of misfolded proteins for degradation, we queried the expression of their ERAD-associated co-factors, ERDJ4, ERDJ5, and EDEM1, respectively. ERdj4 and Edem 1 transcripts were significantly increased in $S i l 1^{\mathrm{Gt}}$ quadriceps when compared to age-matched Sill ${ }^{\mathrm{WT}}$ tissue, particularly at the later time point (Figure 3-10A). Transcripts for Herp/Herpudl, a component of the retrotranslocon, displayed a slight increase, which was statistically significant, as did HERP protein levels (data not shown). Together these data indicate an increased demand for the disposal of misfolded ER proteins. Improperly matured or aggregated ER proteins, as well as damaged ER, can also be disposed of by lysosomal and autophagy pathways. In keeping with a role for these systems in managing ER proteostasis failures, we detected a relative increase in transcription factor EB (TFEB), a key mediator of lysosomal biogenesis, as early as 6 months (Figure 3-10B, E). Consistent with this, we detected multiple TFEB-targets that play a role in lysosomal function to be significantly upregulated by our proteomics study (Figure 3-11A and Supplementary Table S2). We validated one of these, the mannose- 

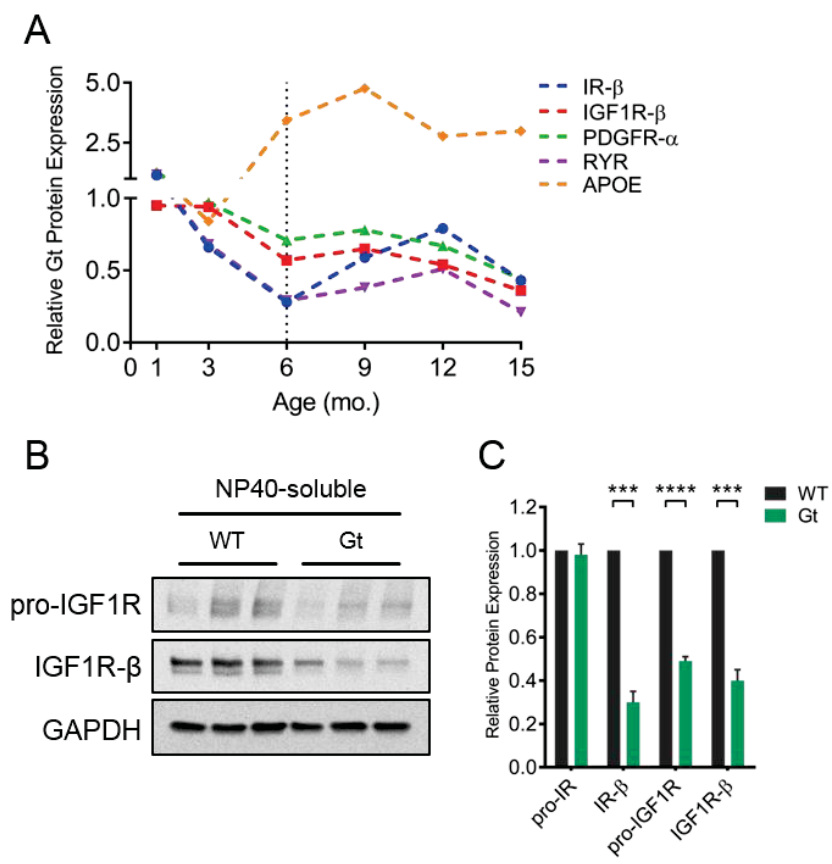

Figure 3-9. Loss of SIL1 leads to disruption of ER proteostasis.

(A) Graphs represent densitometric quantitation of western blotting data from Figure 37C. Dotted vertical line at the 6-month time point indicates the age of onset of muscular weakness in Sill ${ }^{\mathrm{Gt}}$ mice and at which quantitative proteomic profiling of quadriceps was carried out. (B) NP40-solubilized quadriceps lysates from 12-month-old mice were processed for western blotting to detect the IGF-1 receptor precursor (pro-IGF1R) and the mature subunit (IGF1R- $\beta$ ). (C) Graph represent densitometric quantitation of western blotting data from Figure 3-7D and Figure 3-9B $(n=3)$. Error bars indicate means \pm s.e.m. Statistical differences were computed using unpaired, two-tailed Student's $t$-tests, and are indicated as $* * * P \leq 0.001$ and $* * * * P \leq 0.0001$. 
A

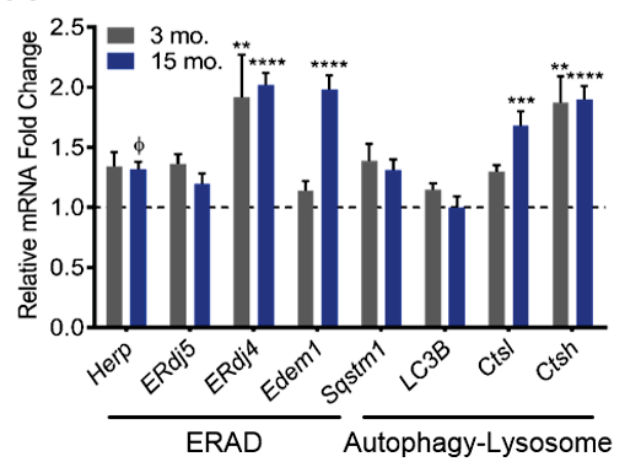

C

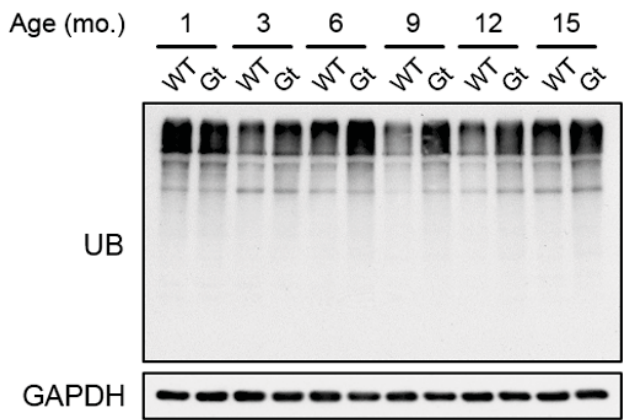

$\mathrm{E}$

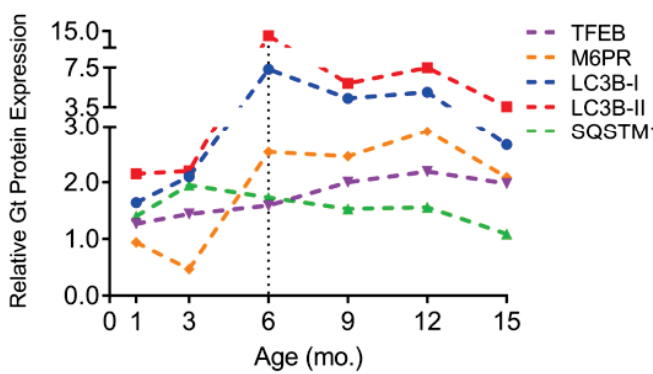

B

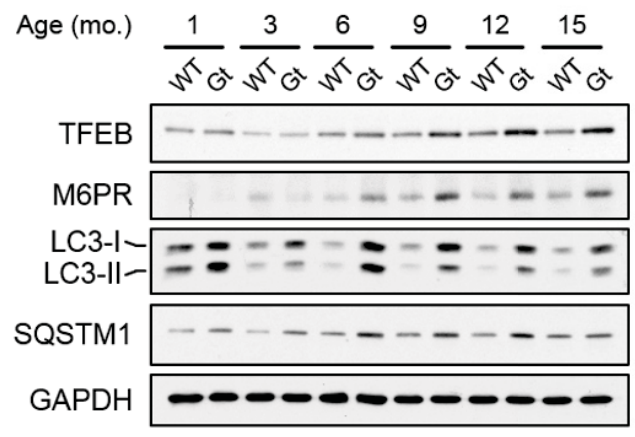

$\mathrm{D}$

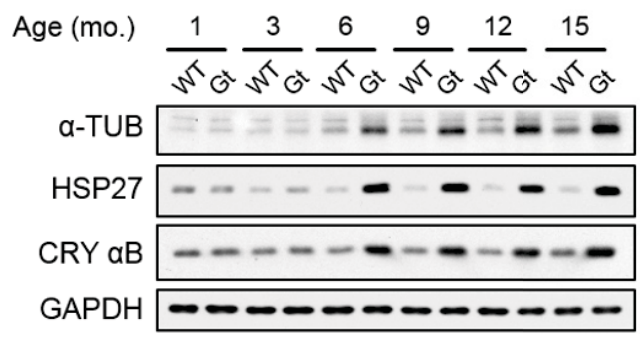

$\mathrm{F}$

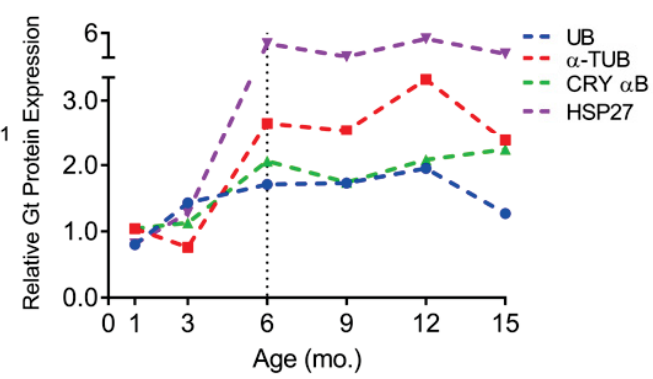

Figure 3-10. Loss of SIL1 results in compensating increases in all cellular protein degradation systems.

(A) Graph of normalized mRNA fold changes of ERAD components (Herp, ERdj5, ERdj4, and Edem 1), mediators of autophagy (Sqstm 1/p62 and LC3B), and lysosomal hydrolases [cathepsin L $(C t s l)$ and cathepsin H $(C t s h)$ ] in quadriceps $(n=3)$ obtained at 3 (gray) and 15 (blue) months of age, respectively. Error bars indicate means \pm s.e.m. Statistical differences were computed using unpaired, two-tailed Student's $t$-tests, and are indicated as ${ }^{\phi} P<0.1$ but $>0.05, * * P \leq 0.01$, $* * * P \leq 0.001$, and $* * * * P \leq 0.0001$. (B, C) Urea-lysed, pooled cellular extracts $(n=3-5)$ isolated at the indicated ages were probed for markers of autophagy and lysosomal biogenesis (B), and also for the accumulation of ubiquitinated proteins (C) by western blotting. GAPDH is a control for equivalent loading in both panels. (D) Lysates were probed for the indicated proteins involved in sequestering misfolded proteins and maintaining the cytoskeleton. (E, F) Graphs represent densitometric quantitation of western blotting data from Figure 3-10B-D. 
A

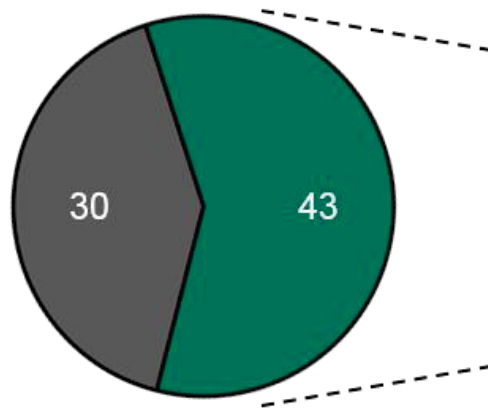

14

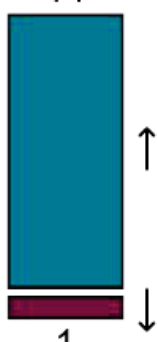

TFEB-regulated proteins involved in autophagy and lysosomal function
B

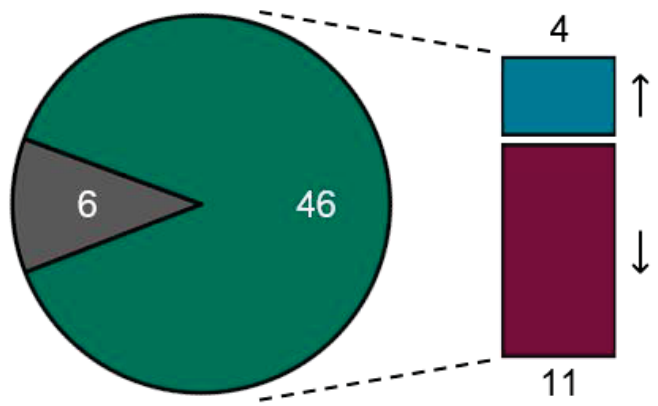

TFEB-regulated proteins involved in mitochondrial biogenesis
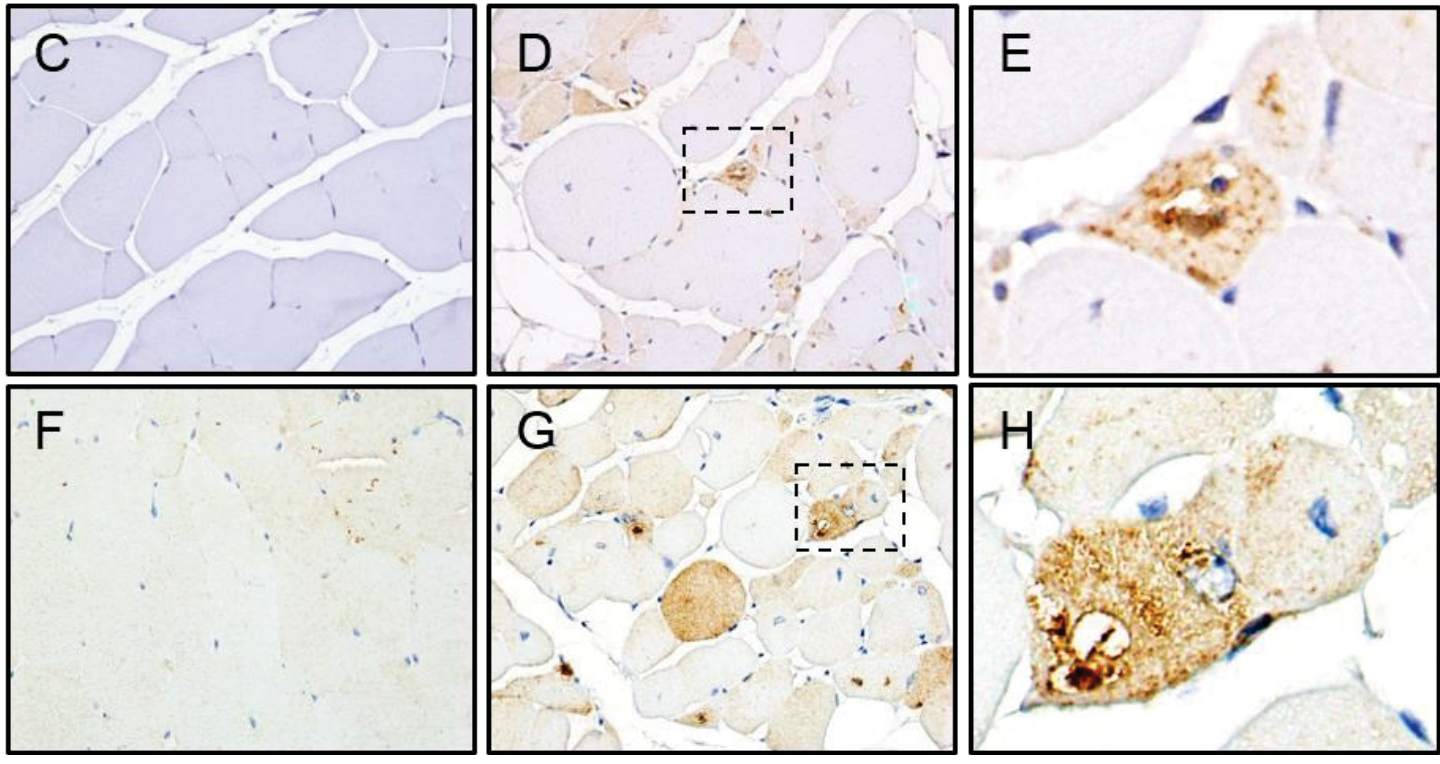

Figure 3-11. Loss of SIL1 leads to aggregation of cytosolic small heat shock chaperones and disruption of cytosolic proteostasis.

(A, B) Graphical representation of TFEB-regulated genes that were implicated in (A) autophagy and lysosomal function or in (B) mitochondrial biogenesis. The total number of genes annotated in each study is represented by green and grey segments of each pie chart. The green segment of these pie charts (Supplementary Table S2) represents the fraction of these TFEB-regulated genes whose proteomic signature was detected in our proteomics study. The bar chart adjacent to it displays the number of proteins significantly $\left(\log _{2}\right.$ ratio $<-0.25$ or $\left.>0.25 ; P<0.01\right)$ upregulated (blue) or downregulated (red) in $\mathrm{Sill}^{\mathrm{Gt}}$ quadriceps, compared to wild-type muscles. (C-H) Representative hindlimb skeletal muscle cross-sections derived from 12-month-old wild-type (C, F) and $\operatorname{Sill}^{\mathrm{Gt}}(\mathrm{D}, \mathrm{E}, \mathrm{G}, \mathrm{H})$ mice are stained with markers of autophagy, LC3B (C-E) and p62 (F$H)$. $(E, H)$ Display a higher magnification of the indicated area from $(D, G)$, respectively. 
6-phosphate receptor (Figure 3-10B, E), which transports lysosomal enzymes and clients to lysosomes (141). Transcripts for cathepsin L and cathepsin H, two lysosomal hydrolases, were also significantly increased (Figure 3-10A). TFEB over-expression has also been reported to drive mitochondrial biogenesis (142). Although our proteomics data captured 46 TFEB-regulated proteins involved in this process, most were either unchanged or diminished (Figure 3-11B), arguing that TFEB did not apparently reach sufficiently high levels to contribute to their expression. Proteins and damaged organelles are also delivered to the lysosome via autophagy. Both the unmodified and lipidated forms of the microtubule-associated protein 1 light chain 3 beta (LC3B), the latter of which initiates the formation of autophagic vesicles, were significantly upregulated in Sill ${ }^{\mathrm{Gt}}$ quadriceps at even the earliest time point queried. Similarly, we observed a modest increase in the levels of the autophagic adaptor protein, SQSTM1/p62, which promotes the sequestration of ubiquitinated target proteins into autophagosomes, leading to lysosomal degradation of both p62 and associated proteins (Figure 3-10B, E). This increase in the protein levels of LC3B and p62 was not due to a corresponding increase in their transcripts (Figure 3-10A). In addition, we detected the presence of LC3B- and p62-positive puncta in Sill $^{\text {Gt }}$ skeletal muscles by immunohistochemistry (Figure 3-11C-H). Thus, the accumulation of unmodified LC3-I and p62 by western blotting, cellular aggregation of these two proteins in puncta, and build-up of autophagosomes observed by EM together indicate impaired autophagy, which precedes the stimulus for lysosomal biogenesis in Sill ${ }^{\text {Gt }}$ skeletal muscles.

Although our data show an up-regulation of components involved in cellular clearance pathways in response to SIL1 disruption, this was apparently not sufficient to dispose of all misfolded proteins, as Sill ${ }^{\mathrm{Gt}}$ quadriceps samples also displayed a greater accumulation of ubiquitinated proteins relative to $S i l 1^{\mathrm{WT}}$ samples beginning at 3 months of age and extending throughout the time course measured (Figure 3-10C, F). This taxation of the ubiquitin-proteasome system likely also compromises the turnover of proteins in other organelles including the cytosol, as indicated by the up-regulation of two cytosolic small heat shock proteins (sHSP), HSP27 and $\alpha \mathrm{B}-$ Crystallin (Figure 3-10D, F), coinciding with the accumulation of ubiquitinated proteins in the Sill ${ }^{\mathrm{Gt}}$ samples. In addition to their primary roles in chaperoning multiple cytoskeletal proteins, these sHSPs play a more general cytoprotective role by sequestering cytosolic protein aggregates (143), which is in keeping with the presence of these sHSP chaperones in the NP40insoluble fraction (Figure 3-12) in Sill ${ }^{\mathrm{Gt}}$ quadriceps. We also observed an increase in the cytoskeletal proteins, $\alpha$-tubulin (Figure 3-10D) and desmin (Figure 3-6B and Figure 3-12), which rely on sHSPs to fold correctly (144). If the protein folding capacity of these sHSPs becomes limiting, it is likely to impinge on client folding, consistent with desmin aggregation in Sill $^{\mathrm{Gt}}$ skeletal muscles (Figure 3-3F).

\section{Disruption of SIL1 has profound effects on components of the insulin signaling pathway}

Insulin and IGF-1 signaling in skeletal muscles helps regulate systemic glucose homeostasis and maintain muscle mass [reviewed in $(145,146)]$. These hormones signal 

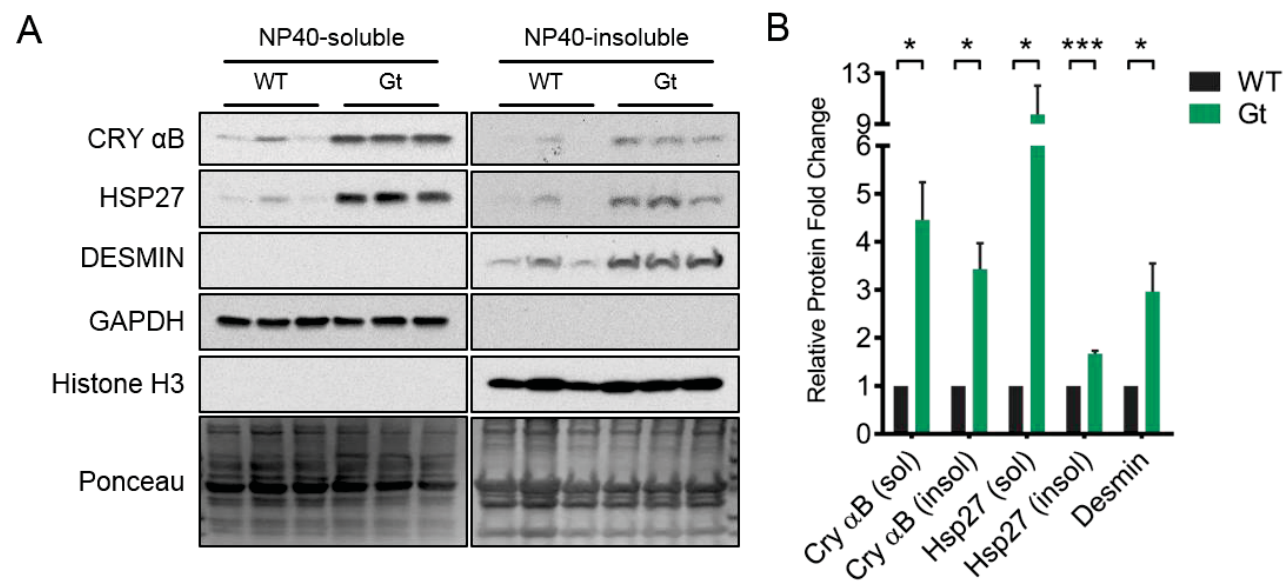

Figure 3-12. Loss of SIL1 leads to aggregation of cytosolic small heat shock chaperones and disruption of cytosolic proteostasis.

(A) Wild-type and Sill ${ }^{\mathrm{Gt}}$ quadriceps obtained from 12-month-old mice were lysed with NP40 lysis buffer. The resulting NP40-soluble and NP40-insoluble fractions were processed for western blotting for the indicated proteins. GAPDH and histone H3 serve as markers of NP40-mediated lysis efficiency. The Ponceau stained western blot serves as the loading control. (B) Graph represents densitometric quantitation of western blotting data from Figure 3-12A $(n=3)$. Error bars indicate means \pm s.e.m. Statistical differences were computed using unpaired, two-tailed Student's $t$-tests, and are indicated as $* P \leq 0.05$ and $* * * P \leq 0.001$ 
through two highly homologous tyrosine kinase receptors that activate the PI3K-AKT pathway (Figure 3-13A). We had verified that both IR- $\beta$ and IGF1R- $\beta$ expression were decreased in the Sill $^{\mathrm{Gt}}$ quadriceps beginning at 6 months (Figure 3-7C). Our proteomic data suggested that this decrease was accompanied by changes in the expression of numerous downstream components of the IR/IGF1R signaling pathway, including an unexpected increase in the levels of total AKT (Figure 3-5D). We also observed reduced levels of key regulatory enzymes of glucose metabolism, including those involved in gluconeogenesis, glyconeogenesis, glycolysis, and the pentose phosphate pathway (Figure 3-5E). Thus, we examined the IR/IGF1R signaling pathway more closely in quadriceps from ad libitum-fed mice, which were the source of all our studies to this point. Paradoxically, we observed increased phosphorylation of their downstream target, PI3K, on tyrosine 199 beginning at 6 months and continuing through later time points (Figure 3-13B and Figure 3-14C, D). To better understand the significance of PI3K activation, we focused on the phosphorylation status of a number of both direct and indirect effectors of this kinase (147). First, due to their respective contribution to skeletal muscle atrophy and translation repression, the FOXO transcription factors and 4E-BP1 were examined. A relative increase in phosphorylation was observed for FOXO1, FOXO3a, and 4E-BP1 on the AKT- and mTOR-dependent residues, respectively, which in each case suppresses their activity. Reduced transcriptional activity of FOXO is further supported by decreased levels of two of its targets, the E3 ubiquitin ligases, Atrogin-1 and MuRF1, at the latter time point (Figure 3-15A). Correspondingly, we observed a striking increase in Fgf21 mRNA (Figure 3-15B), an AKT-regulated myokine that has been implicated in metabolic adaptations to autophagy inhibition and ER stress (148). Together, these data demonstrate the aberrant increase in $\mathrm{PI} 3 \mathrm{~K}$ activation in the Sill $^{\mathrm{Gt}}$ skeletal muscle is driving downstream responses, which are likely to limit the loss of muscle strength and mass.

Despite a sustained increase in PI3K-AKT signaling, the Sil1 ${ }^{\mathrm{Gt}}$ mice continued to lose muscle mass and strength as they aged. Although we did not observe evidence of an overt mitochondrial phenotype in these mice, impairment in energy production can lead to myopathy. Thus, the pronounced activation of AMPK, an energy stress sensor, can inhibit mTOR-mediated signaling, leading to a decrease in skeletal muscle mass. We checked the activation status of AMPK and its downstream target, Acetyl-CoA carboxylase (ACC). Although there was a modest increase in AMPK phosphorylation at two time points, this did not correspond to a significant increase in phosphorylation of its target, ACC (Figure 3-14B, E), suggesting that decreased energy production was not a significant driver of this myopathy. We also examined transcripts of GADD45A, a critical mediator of skeletal muscle atrophy, because it can be up-regulated by the PERK pathway (149), which is in keeping with our observation that CHOP, another PERK target, was induced (Figure 3-8B, C). Indeed, there was a significant increase in Gadd45a mRNA in Sill ${ }^{\text {Gt }}$ quadriceps as early as 3 months, which continued to climb as these mice aged, as well as a modest increase in Ncam1, a marker of atrophy (Figure 3-15A). Two other mediators of atrophy, Atrogin-1 and MuRF1, were not induced, likely due to the suppression of FOXO by AKT, thus resulting in a dampened atrophic response. This, coupled with the absence of a regeneration driven by an increase in PAX7, MYOD1, and myogenin (Figure 3-15A), apparently cause the slow, progressive 


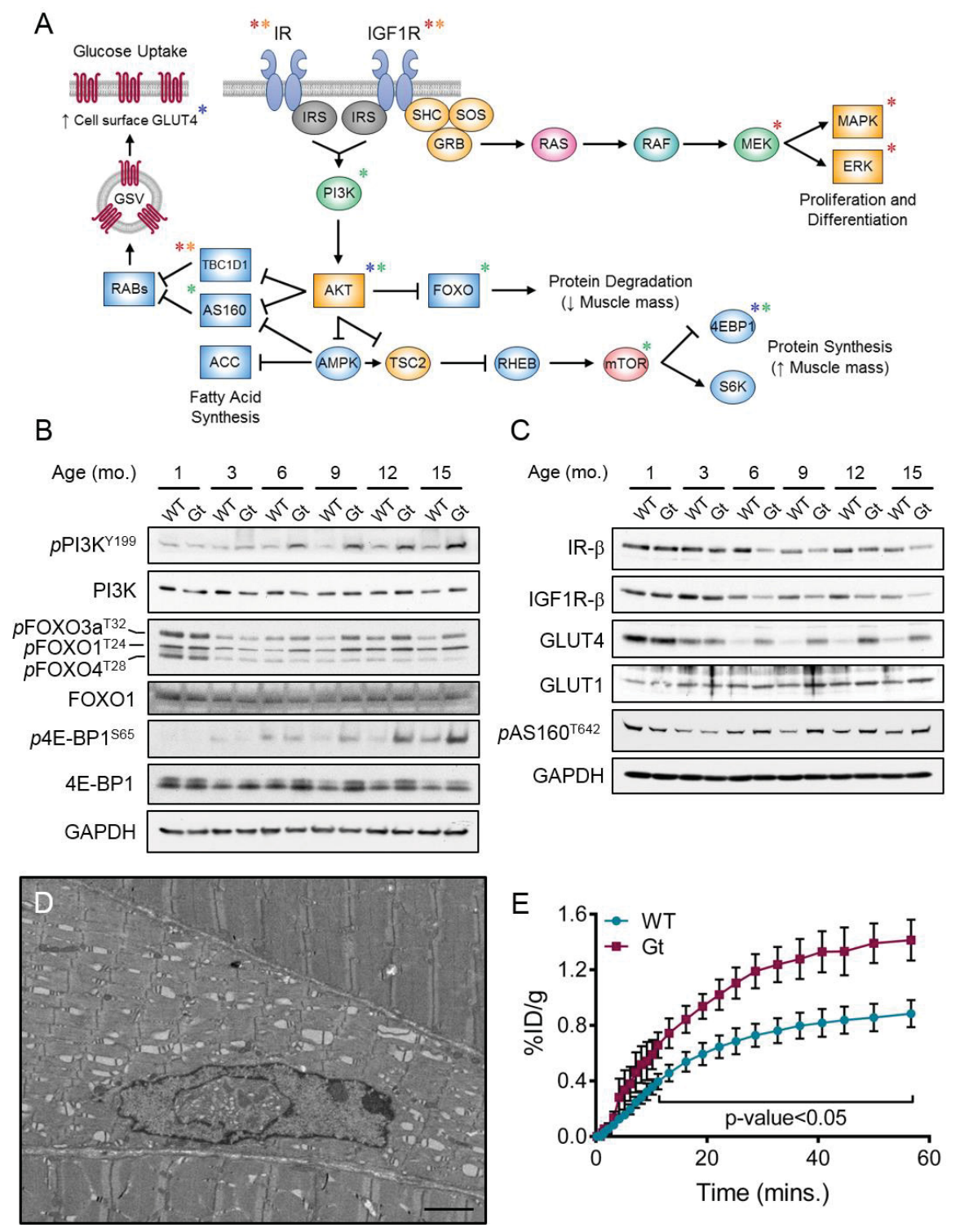

\section{Figure 3-13. Loss of SIL1 has profound effects on IR signaling.}

(A) Schematic of the IR/IGF1R signaling pathway. Asterisks indicate proteins identified to be decreased by proteomics (red *), western-blotting-validated decreases (orange *) and increases (blue *), and proteins displaying increased basal phosphorylation (green *), relative to Sill ${ }^{\mathrm{WT}}$ levels. (B, C) Pooled, urea-solubilized, quadricep lysates derived from mice at the depicted ages $(n=3-5)$ were analyzed by western blotting with antibodies against multiple components of the IR- and IGF1R-signaling pathways. GAPDH serves as a control for equivalent loading. (D) Representative gastrocnemius electron micrograph from 10-month-old Sill ${ }^{\mathrm{Gt}}$ mice displaying myopathic alterations very similar to those observed in the quadriceps, including degenerating nuclei and dilated triads. Scale bar: $2 \mu \mathrm{m}$. (E) Graphical representation of ${ }^{18} \mathrm{~F}$-fluorodeoxyglucose $\left({ }^{18} \mathrm{~F}-\mathrm{FDG}\right)$ uptake over 60 minutes in wild-type (blue) and $\mathrm{Sill}^{\mathrm{Gt}}$ (red) gastrocnemii $(n=4)$ assayed by PET- $\mu C T$. Statistical differences were computed using unpaired, two-tailed Student's $t$ tests, and are indicated in the graph. $\% \mathrm{ID} / \mathrm{g}, \%$ injected dose per gram body weight. 
A

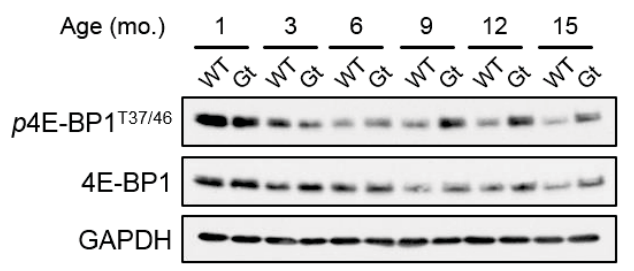

C

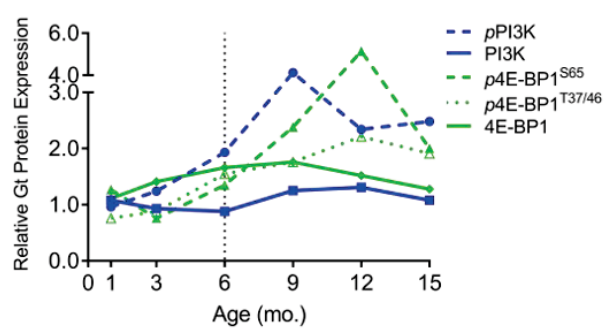

$\mathrm{E}$

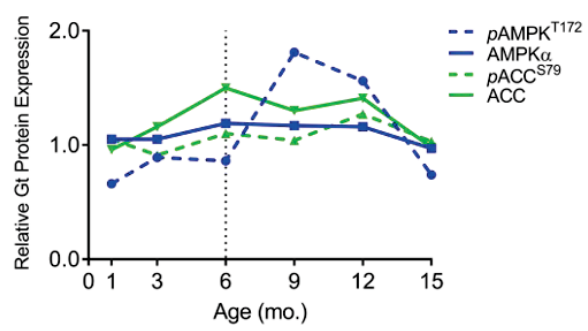

B

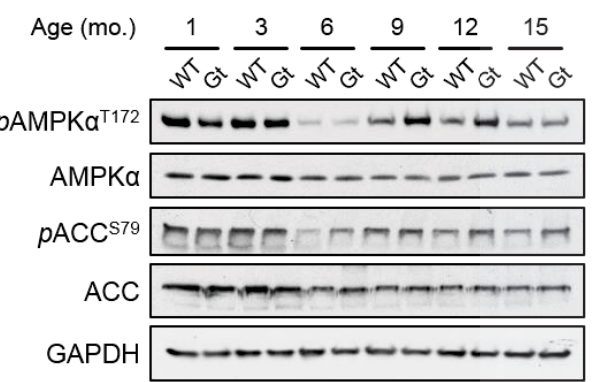

D

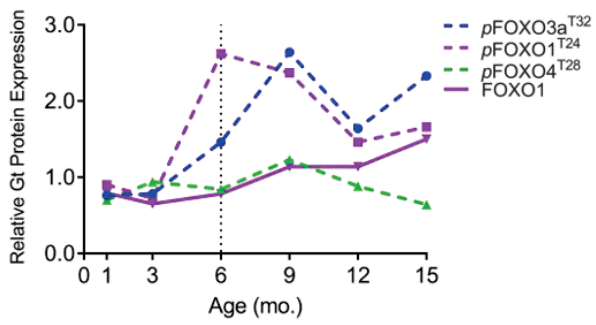

$\mathrm{F}$

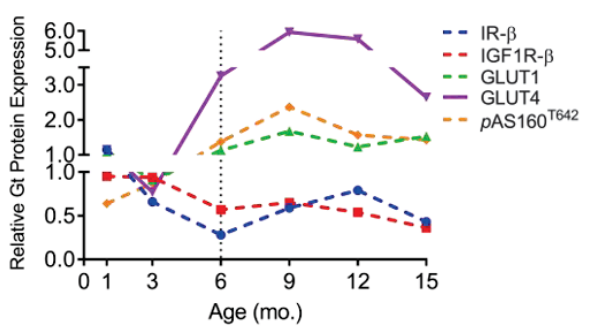

Figure 3-14. Loss of SIL1 leads to basal activation of growth factor signaling.

(A, B) Urea-solubilized quadriceps lysates derived from mice of the indicated genotypes and ages were probed with antibodies against phosphorylated 4E-BP1 downstream of PI3K-AKT-mTOR signaling (A), and phosphorylation-mediated activation of AMPK and ACC (B). (C-F) Graphs represent densitometric quantitation of western blotting data from Figure 3-13B, C and Figure 3-14A, B. 
A

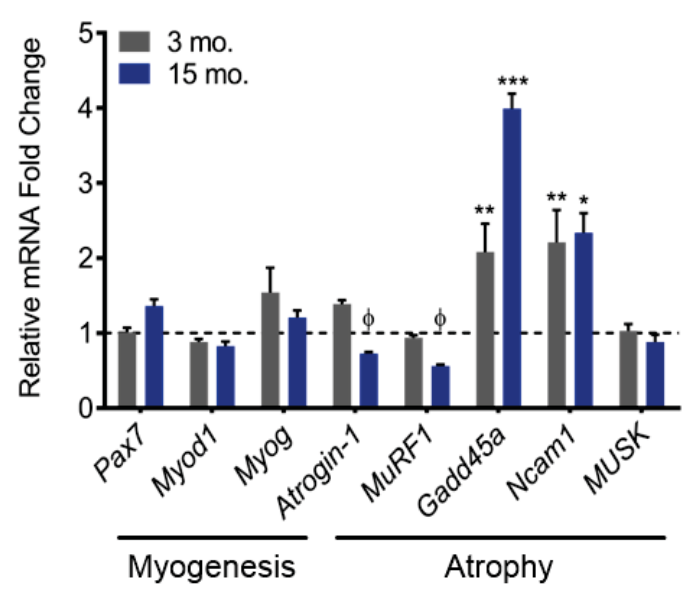

$\mathrm{B}$

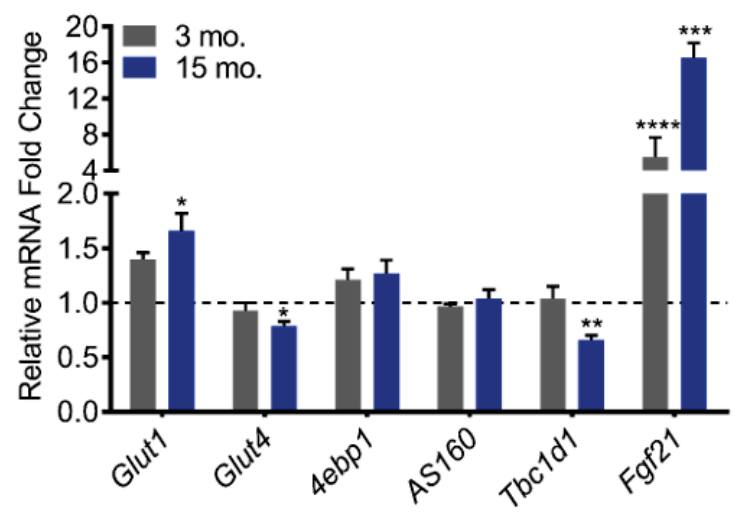

Figure 3-15. Evaluating the expression of genes involved in skeletal muscle regeneration, atrophy, and glucose uptake.

$(\mathrm{A}, \mathrm{B})$ Graphical representation of normalized mRNA fold changes in Sil1 ${ }^{\mathrm{Gt}}$ quadriceps $(n=3)$ of genes involved in (A) satellite cell activation (Pax7), early myogenesis (Myod1), terminal myogenesis (Myog), atrophy (Atrogin-1, MuRF1, Gadd45a, Ncam1, and MUSK); or (B) glucose uptake (Glut1, Glut4, AS160/Tbc1d4, Tbc1d1, and Fgf21) at 3 (gray) and 15 (blue) months, respectively. Error bars indicate means \pm s.e.m. Statistical differences were computed using unpaired, two-tailed Student's $t$-tests, and are indicated as ${ }^{\phi} P<0.1$ but $>0.05, * P \leq 0.05, * * P \leq 0.01, * * * P \leq 0.001, * * * * P \leq 0.0001$. 
myopathy observed in Sill ${ }^{\mathrm{Gt}}$ mice.

We also observed a dramatic increase in the levels of GLUT4, the insulinresponsive glucose transporter, beginning at 6 months, whereas GLUT1, which is an insulin-independent glucose transporter, was only modestly increased (Figure 3-13C and Figure 3-14F). The small increase in GLUT1 expression may be due to a similar increase in its transcripts, whereas Glut 4 mRNA levels were actually slightly decreased (Figure 3-15B), suggesting post-translational stabilization of this transporter. A significant pool of GLUT4 is maintained in intracellular vesicles that can be rapidly deployed to the cell surface to allow glucose uptake in response to insulin signaling through the IR (Figure 3-13A). This relocation of GLUT4 is negatively regulated by two GTPase-activating proteins, TBC1D1 and AS160 (TBC1D4), and they are in turn inactivated by phosphorylation through the insulin signaling pathway [as reviewed in (150)]. We observed a slight steady state increase in phospho-AS160 beginning at 6 months (Figure 3-13C) and a significant decrease in Tbcldl transcripts in our 15-month samples (Figure 3-15B). Together this indicates a reduction in both negative regulators of GLUT4 transport, arguing for a plausible increase in the cell surface localization of GLUT4 in Sill ${ }^{\mathrm{Gt}}$ quadriceps.

To understand the physiological relevance of increased GLUT4 levels and an inhibition or reduction of the negative regulators of GLUT4-trafficking under steady state conditions, we measured basal glucose uptake rate in skeletal muscles using ${ }^{18} \mathrm{~F}$-labeled fluorodeoxyglucose $\left({ }^{18} \mathrm{~F}-\mathrm{FDG}\right) \mathrm{PET}-\mu \mathrm{CT}$. Due to technical limitations, we were unable to analyze the signal originating from quadriceps, our tissue of choice. Thus, as an alternative, we monitored ${ }^{18} \mathrm{~F}$-FDG uptake in gastrocnemii, which are also primarily glycolytic skeletal muscles and display a pathology similar to that observed in quadriceps upon SIL1 disruption (Figure 3-13D). We observed an accelerated rate and increased magnitude of glucose uptake in gastrocnemii of Sill ${ }^{\mathrm{Gt}}$ mice, compared to age-matched wild-type mice (Figure 3-13E). This indicates that the observed increases in basal phosphorylation of multiple components of the AKT pathway indeed represents functional activation of this signaling cascade and likely provides at least some compensation in the face of decreased IR- $\beta$ and IGF1R- $\beta$ protein expression.

\section{Discussion}

Evaluation of the SIL1-deficient quadriceps proteome revealed that loss of SIL1 not only had a large effect on the expression of secretory pathway proteins that are synthesized in the ER, but also led to significant changes in all major cellular organelles, as well as pathways critical for normal muscle physiology. Our interpretation of the causes of these wide-ranging effects on cellular homeostasis is as follows (Figure 3-16). The loss of SIL1, a NEF for the ER chaperone BiP, results in an imbalance in the proteinfolding capacity of the ER, as evidenced by post-translational alterations in the levels of multiple secretory pathway clients and the presence of ER chaperones partially

partitioning in the NP40-insoluble fraction, putatively bound to secretory pathway protein aggregates. This results in the up-regulation of ER chaperones and components of 


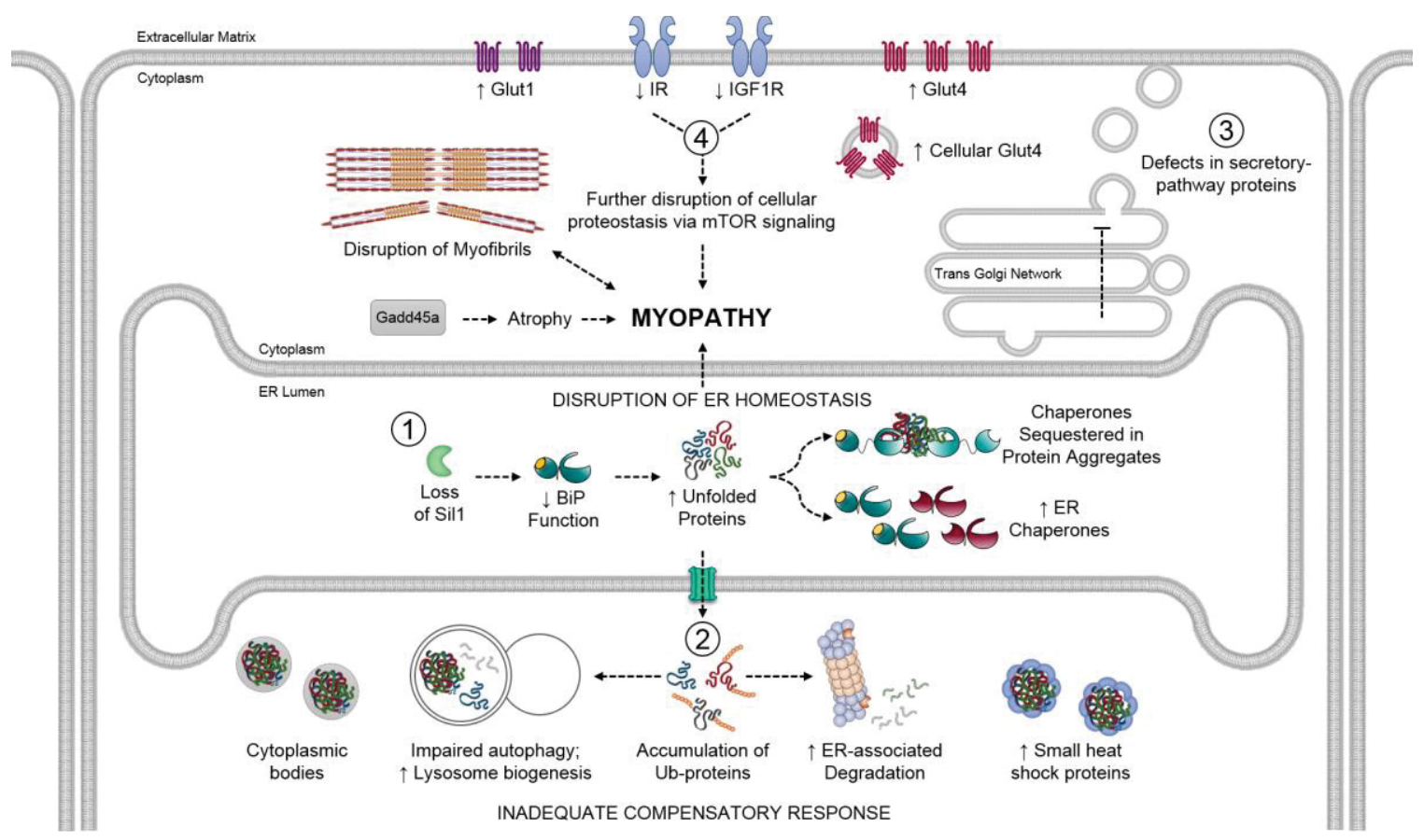

Figure 3-16. Schematic model describing the molecular responses to SIL1 loss in skeletal muscles leading to myopathy.

(1) A functional depletion of SIL1 compromises the chaperoning ability of BiP, leading to an increase in chaperones in an attempt to maintain normal protein folding in the ER. However, this compensation is insufficient, resulting in client misfolding and aggregation. (2) Degradation pathways are up-regulated to deal with accumulating misfolded proteins but are heavily taxed, leading to the build-up of ubiquitinated proteins and induction of small heat shock proteins to sequester aggregates. (3) In keeping with SIL1's role in protein folding, disruption of ER homeostasis adversely affects maturation of secretory pathway clients in the ER. (4) Consequentially, this results in a decrease in the amount of some clients, such as IR- $\beta$ and IGF1R- $\beta$, which play critical roles in maintaining skeletal muscle protein- and glucose-homeostasis. Disruption of normal signaling via these axes, in combination with other factors, such as an increase in GADD45A levels, eventually shifts the balance between anabolic and catabolic cellular mechanisms towards catabolic, atrophy-causing pathway(s) that lead to cyto-architecture disruption as well as decreased muscle mass and strength in the Sil1 ${ }^{\mathrm{Gt}}$ MSS-mouse model. 
multiple cellular protein degradation pathways due to an increased demand for both. However, these responses seemed unable to wholly compensate for an apparent perturbation in ER homeostasis. As a result, cellular protein degradation pathways are heavily taxed, secondarily affecting the turnover of proteins throughout the cell, as evidenced by the accumulation of ubiquitinated proteins, cytosolic sHSP chaperones fractionating in the detergent-insoluble fraction, likely bound to protein aggregates, and the presence of electron-dense material and cytosolic bodies detected by EM. Multiple studies document a basal activation of the UPR in normal adult murine skeletal muscles (151-153), which contributes to myogenesis (154) and adaptation to exercise (155). Thus, the up-regulation of numerous effectors of the UPR observed in our study indicates an increase that exceeds basal activation. However, the basal engagement of the UPR in adult skeletal muscles may restrict the extent to which this pathway can be further upregulated physiologically in the event of a disruption in ER homeostasis, as observed upon SIL1 loss. This would explain why despite UPR activation, the SIL1-deficient ER has not been able to completely resolve its proteostasis defects.

In keeping with a disruption in ER homeostasis, we identified significant decreases in the expression of IR- $\beta$ and IGF1R- $\beta$, which are synthesized in the ER and likely fail to mature properly under these conditions, leading to their disposal by ERAD or lysosomal systems. Although we observed decreases in the up-stream transducers of this pathway, paradoxically we found evidence of higher basal stimulation of downstream components in Sil1 ${ }^{\mathrm{Gt}}$ quadriceps. It is noteworthy that mice with a muscle-specific dual knockout of IR and IGF1R (MIGIRKO) also displayed a compensatory basal activation of the PI3K-AKT-mTOR pathway and increased surface expression of the GLUT1 and GLUT4 glucose transporters (156), confirming that skeletal muscles can respond to decreased (or completely absent) levels of these two receptor tyrosine kinases by constitutively inducing their shared effector pathway. Interestingly, spliced B-boxbinding protein 1 (sXBP1) has been demonstrated to preserve sensitivity of the PI3KAKT signaling axis during high-fat diet induced ER stress by negating the inhibitory effects of IRE1-mediated JNK activation (157). Thus, the concomitant upregulation of $s X b p 1$ in Sill $^{\text {Gt }}$ quadriceps is likely to contribute to the maintenance of a sensitive growth factor signaling axis in the face of decreased IR and IGF1R levels. Skeletal muscle mass is maintained by a dynamic balance between anabolic (hypertrophic increase in protein synthesis rates) and catabolic (atrophic increase in protein degradation pathways)

processes. Signaling through the PI3K-AKT pathway positively regulates muscle growth and mass by increasing cellular protein synthesis through mTOR signaling $(158,159)$ and AKT-dependent phosphorylation of FOXO transcription factors to suppress their atrophy-inducing activity. Although we observed both these cues in Sill ${ }^{\mathrm{Gt}}$ quadriceps, muscle mass continued to decline, suggesting that these counteracting mechanisms were insufficient to prevent atrophy. It is intriguing to hypothesize that the seemingly compensatory activation of mTOR signaling in Sill ${ }^{\text {Gt }}$ skeletal muscles, which would maintain high protein synthesis rates and inhibit autophagy (160), might in fact further exacerbate the demands on an already abnormally taxed protein folding machinery. In support of this hypothesis, there is a growing body of literature that emphasizes the beneficiary effect of reduced PI3K-AKT-mTOR signaling on reducing proteotoxicity associated with protein misfolding diseases in worms and mice $(161,162)$. In keeping 
with this hypothesis, over-expression of the translation inhibitor 4E-BP dramatically suppressed pathological phenotypes in a Drosophila model of Parkinson's Disease (163), and inhibition of mTOR with rapamycin abolished cognitive defects and reduced $\beta$ amyloid levels in a murine Alzheimer's Disease model (164).

Aging is the principal risk factor in various protein folding diseases, such as BAG3-, CRY $\alpha$ B-, and DNAJB6-associated myofibrillar myopathies, as well as familial amyotrophic lateral sclerosis, Alzheimer's, Huntington's, and Parkinson's Diseases, all of which have been characterized as "gain-of-toxic-function" diseases (165). Although all of these diseases result from germline-encoded mutation(s), disease-associated symptoms and even aggregation of the affected proteins does not occur until later in life. This has been attributed to a progressive, age-related, decline in chaperone function, clearance of aberrantly folded proteins, and the ability to mount successful stress responses, all of which collectively maintain the proteome integrity. These changes have been documented in multiple tissue types, including muscles, and in organisms ranging from nematodes to fruit flies and mammals (166). This decline in proteostasis maintenance during aging predicts that, as the buffering capacity of the chaperones and stress responses diminish, misfolded proteins begin to aggregate. Powers et al., have used mathematical modeling to argue that there are limits on the capacity of the proteostasis network in cells and that metastable proteins would be the first to be affected as this network declines (167). Although SIL1 is a component of the ER chaperone machinery, and might be expected to affect the proper maturation of numerous secretory pathway proteins, we also observed alterations in protein expression in other subcellular organelles and a similar delay in the onset of physiological symptoms and molecular responses. Thus, we argue that the resulting disruption of ER function in Sil1 ${ }^{\text {Gt }}$ skeletal muscles provides a sufficient burden to accelerate the usual age-restricted limitations of the proteostasis network. Furthermore, we find that the alterations in ER homeostasis secondarily impinge on proteostasis in other organelles, supporting the hypothesis that organellar boundaries are susceptible to disruption in trans (167).

One might speculate that any of the secretory pathway proteins that show altered expression upon Sill-disruption represent clients that are critically dependent on SIL1 for release from BiP. However, alterations in the expression of the secretory pathway proteins that we examined did not become apparent until the mice were 6 mo. of age. This argues that Sill ${ }^{\mathrm{Gt}}$ mice were able to control the expression of these proteins normally in the absence of SIL1 at earlier time points and raises the question of why they are unable to do so later. It is possible that basal levels of GRP170, another ER NEF for BiP, are initially sufficient to compensate for the loss of SIL1 function but cannot do so at later time points. Alternatively, an intriguing possibility comes from recent studies in yeast showing that Sillp (yeast SIL1) can also act as a reductase for oxidized yeast BiP (Kar2p), thereby restoring Kar2p's chaperone activity during recovery from oxidative stress. Sil1p's reductase activity is mediated through two cysteines present near its Nterminus (66). Although there are two N-terminal cysteines that are highly conserved in mammalian SIL1 sequences, algorithms for signal sequence cleavage predict that they are not retained in the mature protein. If in fact they are part of the mature SIL1 protein, a similar reductase activity for mammalian SIL1 could readily explain the delayed effects 
on secretory pathway protein expression, as the need for this reductase activity might be dependent on accumulated oxidative insults to the ER, leading to the oxidization and inactivation of BiP. Further investigation is required to determine if mammalian SIL1 possesses a reductase activity for BiP and whether this is a plausible explanation for the pathologies associated with MSS.

In toto, our study revealed that SIL1 loss leads to early and persistent defects in ER homeostasis, which invokes a triage response to increase both the protein folding and degradation capacity of the ER, which is ultimately inadequate. One consequence of the inability to restore proper ER function is a post-translational decrease in IR- $\beta$ and IGF1R$\beta$ expression in SIL1-deficient skeletal muscles. While we observed evidence of a compensatory increase in basal PI3K-AKT signaling and expression of glucose transporters in Sil1 ${ }^{\text {Gt }}$ skeletal muscles, these mice were not able to successfully counteract the activation of atrophy-causing degradation pathways. The physiological requirement of constitutive UPR activation in skeletal muscles, combined with the proteotoxicity associated with increased mTOR signaling and the age-associated decline in the ability to maintain proteostasis, particularly in the absence of a pronounced regenerative response is likely to trigger the loss of skeletal muscle mass and strength upon loss of SIL1. Together, these findings provide molecular insights into the progressive myopathy and cellular triage responses attempted upon loss of an ERQC component.

\section{Materials and Methods}

\section{Mice}

The Sil1 ${ }^{\text {Gt }}$ mouse strain B6;129Sil1 ${ }^{\text {Gt (pGT2TMpfa)1Slac }}$ was generated by gene-trap methodology (87) and was obtained from Dr. Susan Ackerman (HHMI and University of California San Diego). Heterozygous mice were crossed to generate homozygous male and female Sill $^{\mathrm{Gt}}$ mice and wild-type littermates. At weaning, DNA was isolated from mouse tail snips using DNeasy ${ }^{\circledR}$ Blood \& Tissue Kit (Qiagen, Valencia, CA) and genotyped as previously reported (87). The primers for the wild-type allele and for the disrupted allele are mentioned in the Supplementary Table S3. Mutant mice were born at expected Mendelian ratios. Mice were housed and treated in accordance with the Animal Use and Care Committee and the Animal Research Center at St. Jude Children's Research Hospital, adhering to the National Institutes of Health guidelines.

\section{Inverted screen test}

To measure the muscular strength of male and female Sill ${ }^{\mathrm{Gt}}$ and wild-type mice, we performed an inverted screen test (168) using a 12 " x 8 " screen $(0.5$ " metal grid) that was surrounded by 9" plexiglass walls to prevent mice from climbing onto the top of the screen during the assay. A pillow was placed below the apparatus to cushion the fall. Each mouse was placed on the screen, allowed to acclimatize for a few seconds, and the 
screen was rotated $180^{\circ}$ to position the mouse upside down, at which point timing began. The length of time that each mouse was able to hold on to the screen was recorded with an assay maximum limit of $120 \mathrm{~s}$. Each mouse was subjected to a total of three trials successively and the average was determined. Mean times for wild-type and Sill ${ }^{\mathrm{Gt}}$ mice of each age-group were statistically compared using Prism Ver. 6 (GraphPad Software, La Jolla, CA) by Mann-Whitney tests (unpaired; two-tailed).

\section{Histopathology and immunohistochemistry}

Immediately after euthanization, mice were perfused with $10 \%$ buffered Formalin (Thermo Fisher Scientific, Waltham, MA) through the left cardiac ventricle. Skeletal muscles and spinal cords of perfused mice were post-fixed by immersion in $10 \%$ buffered Formalin for at least 24 hours before being decalcified in formic acid (TBD-2 Decalcifier; Thermo Fisher Scientific). Tissues were embedded in paraffin, sectioned at $4 \mu \mathrm{m}$, and mounted on positively charged glass slides (Superfrost Plus; Thermo Fisher Scientific). Tissue sections were processed for IHC using the automated Discovery Ultra platform (Ventana-Roche, Tucson, AZ) after heat-induced antigen retrieval for up to 48 mins. using the Discovery Cell Conditioning CC1 buffer, which included Proteinase K treatment in the case of Laminin IHC. Sections were then stained with the respective primary and secondary antibodies, counter-stained with Hematoxylin II followed by treatment with Bluing Reagent, and detected using the DISCOVERY ChromoMap DAB Kit (RUO) alone or in conjunction with the DISCOVERY Purple kit (RUO) for the double-staining of MyHC fast and slow isoforms.

\section{Transmission electron microscopy}

For ultrastructural analysis by EM, post-euthanization the animals were perfused with $2.5 \%$ glutaraldehyde/ $2 \%$ paraformaldehyde in $0.1 \mathrm{M}$ sodium cacodylate buffer, $\mathrm{pH}$ 7.4 (EM fixative). Necropsy was performed to harvest major skeletal muscles and heart, and submitted to the St. Jude CTI-EM shared resource. The samples were fixed with EM fixative overnight and post fixed in $2 \%$ osmium tetroxide in $0.1 \mathrm{M}$ cacodylate buffer with $0.3 \%$ potassium ferrocyanide for 1.5 hours. After rinsing in the same buffer, the tissue was stained in $4 \%$ aqueous uranyl acetate, and dehydrated through a series of graded ethanol-to-propylene oxide transitions. The samples were then infiltrated in a series of propylene oxide and EPON resin gradients, with processing through 100\% EPON resin overnight. All processing was performed using a Leica EM TP Tissue Processor. The following day, tissues were embedded using fresh EPON resin and polymerized at $70^{\circ} \mathrm{C}$ overnight. Semi-thin sections $(0.5 \mu \mathrm{m})$ were stained with toluidine blue for light microscopy-examination. Ultrathin sections $(80 \mathrm{~nm})$ were cut and imaged using the Jeol 1200 Electron Microscope with a 2K AMT 2K Digital Camera. 


\section{Mass spectrometry}

\section{Protein digestion and peptide isobaric labeling by Tandem Mass Tags (TMTs)}

Mass spectrometry was performed as previously described (169) with a slight modification. Quadriceps tissues from five Sil1 ${ }^{\mathrm{WT}}$ and five Sill ${ }^{\mathrm{Gt}}$ 6-month-old male mice were lysed in a $50 \mathrm{mM}$ HEPES (pH 8.5)-based buffer containing $8 \mathrm{M}$ urea and $0.5 \%$ sodium deoxycholate. Protein concentration of lysates was determined by a Coomassiestained short gel with bovine serum albumin as standard. $100 \mu \mathrm{g}$ of protein for each sample was digested with LysC (Wako) at an enzyme-to-substrate ratio of 1:100 (w/w) for 2 hours at room temperature. Following this, the samples were diluted to a final concentration of $2 \mathrm{M}$ urea in $50 \mathrm{mM}$ HEPES ( $\mathrm{pH} 8.5$ ), and further digested with Trypsin at an enzyme-to-substrate ratio of 1:50 (w/w) for at least 3 hours. The digestion was terminated and acidified by adding trifluoroacetic acid to $1 \%$, desalted using $\mathrm{C} 18$ cartridges, and dried by SpeedVac. The purified peptides were resuspended in $50 \mathrm{mM}$ HEPES (pH 8.5), labeled with 10-plex TMT reagents, following the manufacturer's recommendation (ThermoFisher Scientific).

\section{Two dimensional HPLC and mass spectrometry}

The TMT-labeled samples were mixed in an equal proportion, desalted, and fractionated on an offline high-performance liquid chromatography (HPLC) apparatus (Agilent 1220) using basic $\mathrm{pH}$ reverse phase liquid chromatography ( $\mathrm{pH}$ 8.0, XBridge C18 column, $4.6 \mathrm{~mm} \times 25 \mathrm{~cm}, 3.5 \mu \mathrm{m}$ particle size). The fractions were dried and resuspended in 5\% formic acid and analyzed by acidic $\mathrm{pH}$ reverse phase LC-MS/MS analysis. The peptide samples were loaded on a nanoscale capillary reverse phase $\mathrm{C} 18$ column (New Objective, $75 \mu \mathrm{m}$ ID x $\sim 40 \mathrm{~cm}, 1.9 \mu \mathrm{m}$ C18 resin from Dr. Maisch $\mathrm{GmbH}$ ) by a HPLC system (Waters nanoAcquity) and eluted by a 150 minute-gradient. The eluted peptides were ionized by electrospray ionization, and detected by an inline Orbitrap Fusion mass spectrometer (ThermoFisher Scientific). The mass spectrometer is operated in data-dependent mode with a survey scan in Orbitrap $(60,000$ resolution, $2 \mathrm{X}$ 106 AGC target and $50 \mathrm{~ms}$ maximal ion time) and MS/MS high resolution scans $(60,000$ resolution, $1 \mathrm{X} 105 \mathrm{AGC}$ target, $\sim 150 \mathrm{~ms}$ maximal ion time, $38 \mathrm{HCD}$ normalized collision energy, $1 \mathrm{~m} / \mathrm{z}$ isolation window, and 20 s dynamic exclusion).

\section{Mass spectrometry data analysis and bioinformatics}

The MS/MS raw files were processed by a newly developed tag-based hybrid search engine, JUMP, which displayed better sensitivity and specificity than commercial package (e.g. Proteome Discoverer) (170). The data was searched against the UniProt mouse database concatenated with a reversed decoy database for evaluating false discovery rate. Searches were performed using a $25 \mathrm{ppm}$ mass tolerance for precursor ions and $15 \mathrm{ppm}$ mass tolerance for fragment ions, fully tryptic restriction with two maximal missed cleavages, three maximal modification sites, and the assignment of $a, b$, 
and $y$ ions. TMT tags on lysine residues and N-termini (+229.162932 Da) were used for static modifications and Met oxidation $(+15.99492 \mathrm{Da})$ was considered as a dynamic modification. MS/MS spectra were filtered by mass accuracy and matching scores to reduce protein false discovery rate to $\sim 1 \%$. Proteins were quantified by summing reporter ion counts across all matched peptide spectrum matches using the JUMP software suite.

Bioinformatics analyses were performed to determine significant protein expression, gene ontology (GO), and pathway representation. Using Partek Genomics Suite 6.6 (Partek Incorporated, St Louis, MO), the values of 5384 quantified proteins were $\log _{2}$ transformed followed by quantile normalization and compared using ANOVA between five replicates in each of the two groups. Expressions of a total of 515 proteins were identified as significantly altered $\left(P<0.01 ; \log _{2}\right.$ ratio $<-0.5$ or $\left.>0.5\right)$ in the Sill $^{\mathrm{Gt}}$ quadriceps relative to those from age-matched wild-type mice. DAVID (https://david.ncifcrf.gov/) Bioinformatics Resources 6.7 was then used for GO analysis. Annotations for cellular components were only available only for 490 proteins out of 515, which were then manually curated using the GeneCards database (www.genecards.org) to compute the percentage of proteins belonging to major cellular compartments according to their score. Gene Set Enrichment Analysis (GSEA, http://software.broadinstitute.org/gsea/) software was further performed to identify significant enrichments in differentially expressed gene signatures, including pathways based on gene-sets defined from KEGG, BioCarta, and Reactome pathway databases.

\section{Western blotting}

Male Sil1 ${ }^{\mathrm{Gt}}$ and wild-type mice ( $n=3$ for each genotype and age-group, with the exception of the 6-month time point, for which five male mice of each genotype were used) were used for western blotting studies. Immediately after euthanization, mice were perfused with ice-cold PBS through the left cardiac ventricle. Quadriceps were harvested at necropsy and flash frozen in liquid nitrogen and either processed immediately or stored until further use. The frozen tissue was pulverized using a liquid nitrogen-chilled mortar and pestle, and stored at $-80^{\circ} \mathrm{C}$ until further use. The pulverized tissue was lysed, as described for mass spectrometry analysis, normalized for equivalent protein concentration using a BCA Protein Assay Kit (Pierce-ThermoFisher Scientific, Rockford, IL), and samples from the indicated number of mice at the same age were pooled. To separate NP40-soluble proteins from -insoluble ones, pulverized tissue was lysed in $50 \mathrm{mM}$ Tris ( $\mathrm{pH} 7.5$ ) buffer containing $150 \mathrm{mM} \mathrm{NaCl}, 0.5 \%$ Nonidet P40, and $0.5 \%$ deoxycholic acid. The clarified lysates obtained after centrifugation at $21,130 \mathrm{~g}$ for 20 minutes were designated as the NP40 soluble fraction. The NP40 insoluble pellets were washed once with NP40 lysis buffer and then solubilized in $50 \mathrm{mM}$ Tris (pH 8) buffer, containing $0.6 \%$ SDS, sonicated for 4 minutes (30s on-30s off cycles), and incubated at $95^{\circ} \mathrm{C}$ for 10 mins to prepare the NP40-insoluble fractions. All buffers contained $0.25 \mathrm{mM}$ phenylmethylsulphonyl fluoride (PMSF), and a protease inhibitor cocktail (Complete, Roche). In all cases, tissue lysates were electrophoresed under reducing conditions on gels ranging from 8 to $16 \%$, or on 4 to $20 \%$ gradient gels (Mini- 
PROTEAN $^{\circledR}$ TGX $^{\mathrm{TM}}$ Precast Protein Gels, Bio-Rad, Inc., Hercules, CA), depending on the size of the examined protein. The antibodies used for western blotting are listed in Supplementary Table S3. HSC70 and GAPDH served as loading controls. Western blots from the pooled samples in each of the figures were quantified using either Image Studio $^{\mathrm{TM}}$ Lite (LI-COR Biosciences, Inc., Lincoln, NE) or ImageQuant ${ }^{\mathrm{TM}} \mathrm{TL}$ (GE Healthcare Life Sciences, Inc., Issaquah, WA). These values were normalized to their respective GAPDH control values and graphically expressed as fold change in Sill ${ }^{\mathrm{Gt}}$ protein expression, relative to the wild-type levels set to 1, at each age. Statistical analyses (Figure 3-9C and Figure 3-12B) were performed compared using Prism Ver. 6 (GraphPad Software, La Jolla, CA) by Student's $t$-tests (unpaired; two-tailed).

\section{Real-time PCR}

Three male Sill $^{\mathrm{Gt}}$ and wild-type mice for each genotype and age-group, respectively, were used for quantitative PCR (qPCR) studies. Total RNA was isolated from pulverized skeletal muscle using RNeasy ${ }^{\mathbb{B}}$ Fibrous Tissue Mini Kit (Qiagen, Inc., Valencia, CA), according to the company protocol. $1 \mu \mathrm{g}$ total RNA was reverse transcribed using the High Capacity cDNA Reverse Transcription Kit (Applied Biosystems, Foster City, CA) and subjected to SYBR ${ }^{\circledR}$ Green chemistry-based qPCR using the QuantStudio 3 Real-time PCR System (Applied Biosystems, Foster City, CA). Primers were obtained from ThermoFisher Scientific. The primer sequences used are listed in Supplementary Table S3. Gapdh, Hprt1, Rer1, Rn18s, and Rpl41 were tested as potential endogenous reference controls, as indicated (171). Gapdh, Rer 1, and Rpl41 were comparable in expression between the wild-type and the Sill ${ }^{\text {Gt }}$ quadriceps and, thus, used as controls. Relative mRNA fold change was computed from the QuantStudiogenerated $C_{t}$ values by using the $2^{-\Delta \Delta C t}$ method. Wild-type mRNA levels were normalized to 1, and Sill ${ }^{\mathrm{Gt}} \mathrm{mRNA}$ levels were plotted as a relative measure of the wildtype levels. Statistical analyses were performed using Prism Ver. 6 (GraphPad Software, La Jolla, CA) by Student's $t$-test (unpaired; two-tailed).

\section{Skeletal muscle glucose uptake}

Small animal PET- $\mu \mathrm{CT}$ was performed on docked Siemens Inveon DPET and micro-CT (Siemens Medical Solutions USA, Inc., Malvern, PA) instruments. Four 6 mo.-old wild-type and Sill ${ }^{\mathrm{Gt}}$ male mice were fasted overnight, anesthetized with $2 \%$ isoflurane, and placed supine on a heated animal bed, following which an intraperitoneal catheter was placed for ${ }^{18} \mathrm{~F}-\mathrm{FDG}$ injection. Whole-body dynamic PET scan was acquired over 60 minutes. ${ }^{18}$ F-FDG $(100-120 \mu \mathrm{Ci}, 200 \mu \mathrm{l})$ was injected via the i.p. catheter $1 \mathrm{~min}$. after initiating scanning. $\mu \mathrm{CT}$ data were acquired using a 2048 × $3072 \mathrm{~mm}$ Field of View with whole body coverage achieved using automatic stitching of two bed positions (36\% overlap). Projections were acquired at $80 \mathrm{kVp}$ and $500 \mu \mathrm{A}$ ( $250 \mathrm{~ms}$ exposure) over a rotation of $180^{\circ}$ (120 steps), providing an isotropic resolution of $107 \mu \mathrm{m}$, and used for PET attenuation correction. PET Images were reconstructed by three-dimensional Ordered Subset Expectation Maximization (OSEM 3D) with binning scheme of 5-20, 10- 
60, 5-180, 5-240, and 2-400 (number of bins-time in seconds). The Inveon Research Workspace (IRW) software was utilized for data processing and image co-registration. Volumes of interests (VOIs) were drawn over the gastrocnemius, heart, and bladder. Data from these VOIs were used to calculate radioactivity accumulation for each animal and plotted versus time. Statistical comparisons of \%ID/g were performed by Student's $t$-tests (unpaired; two-tailed) using Prism Ver. 6 (GraphPad Software, La Jolla, CA). 


\section{CHAPTER 4. MSS-ASSOCIATED MUTATIONS DESTABILIZE SIL1 AND CAN CAUSE MARINESCO-SJÖGREN SYNDROME}

\section{Introduction}

Marinesco-Sjögren Syndrome (MSS; OMIM 248800) is a multisystem, progressive disorder with autosomal recessive inheritance. MSS is clinically characterized by the triad of cerebellar ataxia, bilateral cataracts, and progressive myopathy, but is often accompanied by intellectual disability, impaired physical development, skeletal deformities, and hypergonadotropic hypogonadism (78). The primary cause of MSS has been attributed to mutations in the SIL1 gene $(64,86)$, mapping to the chromosomal locus, 5q31 (85). SIL1 encodes an adenine nucleotide exchange factor (NEF) for the endoplasmic-reticulum (ER)-resident Hsp70 chaperone, $\mathrm{BiP}(63) . \mathrm{BiP}$ is a crucial regulator of ER function where it plays a myriad of roles, including gating the ER translocon that provides a channel for nascent proteins entering the secretory pathway, aiding the folding and assembly of these proteins, recognizing and targeting misfolded proteins for proteasomal degradation, regulating the activation of the ER-localized transducers of the unfolded protein response, and serving as an ER-resident calcium-binding protein (reviewed in (33)).

Similar to other Hsp70 family members, BiP possesses an N-terminal nucleotide binding domain (NBD) and a C-terminal substrate binding domain (SBD), which communicate allosterically via an interdomain linker. The ability of BiP to bind to substrates via its SBD is regulated by the nucleotide-bound state of its NBD. ATP-bound $\mathrm{BiP}$ can readily engage new client proteins as they enter the ER, however, this conformation of $\mathrm{BiP}$ has a high on/off rate that does not provide a stable interaction with substrates. ER-resident DnaJ-like proteins (ERdjs), four of which have also been reported to directly engage client proteins (ERdj3-6), stimulate BiP's inherently low ATPase activity while simultaneously transferring the substrate to BiP. Hydrolysis of ATP to ADP allows closing of the SBD 'lid' and thereby stable association of BiP with its substrate. NEFs interact with BiP's NBD and exert a torsional strain on the nucleotide-binding cleft. This causes disengagement and release of the bound ADP. The binding of a new ATP molecule triggers a conformational change in BiP's SBD that lowers the affinity for the bound substrate and causes its release $(43,45)$. The released substrate can then undergo conformational maturation and traffic along the secretory pathway to its final destination. Two ER resident proteins, SIL1 and GRP170, have been identified that possess nucleotide exchange activity for BiP. SIL1 is ubiquitously expressed in humans and mice and its tissue-specific expression pattern is similar to that of $\operatorname{BiP}(63,64)$. GRP170 has also been reported to function as a bona fide ER chaperone capable of binding a substrate pool overlapping with that of BiP (49). Although the nucleotide exchange activity of SIL1 and GRP170 is mechanistically distinct $(43,45)$, partial redundancy in their function might explain the non-lethal phenotype of SIL1 loss in humans and mouse models of MSS (50). 
SIL1 has an elongated 'kidney-bean'-like shape, which is composed of $16 \alpha$ helices (A1-A16) and lacks any $\beta$-sheets. The central helices A3-A14 form four domains with Armadillo-like repeats (ARM1-ARM4) (45). A crystal structure of Saccharomyces cerevisiae Sil1p in complex with the nucleotide binding domain of BiP homolog, Kar2p, suggests that SIL1 acts as a "wrench" that wraps around lobe IIb of BiP's NBD (constituting SIL1's major BiP-interaction sites) and makes an additional contact with lobe Ib (constituting SIL1's minor BiP-interaction sites). This is consistent with the formerly reported structure of SIL1's cytosolic ortholog, HSPBP1, with domain II of HSP70's NBD. HSPBP1 is also composed of four ARM domains, and available structural data implies that HSPBP1 binding to its cognate HSP70 would induce a major distortion to its NBD, destabilizing the bound the ADP and triggering its release, similarly as the Sil1p-Kar2p interaction $(33,172)$.

To-date, 46 MSS-associated SIL1 mutations have been identified. About threefourths of these mutations are due to frameshifts, splicing defects, or premature truncations that are predicted to cause large deletions, resulting in non-functional and unstable mutant proteins (78). Nine other disease-associated SIL1 mutations arise from missense substitutions or in-frame deletions of only a few amino acids, but each of these mutation results in a disease that is indistinguishable from that caused by mutations that ablate nearly the entire protein (86). However, these SIL1 mutants have not been thoroughly characterized before. The goal of this study is to understand the mechanism(s) by which missense mutations and small deletions associated with MSS lead to a loss of SIL1's function.

\section{Results}

\section{MSS-associated SIL1 mutations affect highly conserved regions, and in most cases are predicted to disrupt regional alpha-helicity}

These nine mutations include six missense mutations, which cause a single amino acid substitution (p.R92W, p.L206Q, p.R298C, p.G312E, p.G312R, and p.L457P), two in-frame deletions of either two or five amino acids, respectively (p.V231_I232del and p.R411_D415del), and one C-terminal premature truncation of six amino acids (p.L456X) (Table 4-1 and Figure 4-1). We shall henceforth refer to these mutations as point mutations for the sake of simplicity. To begin to understand the functional consequences of these nine SIL 1 mutations, we first performed a bioinformatics-based characterization. Alignment of the available species-specific protein sequences of SIL1, ranging from humans to yeast, revealed that each of these mutations affected amino acid residues that are highly conserved across multiple mammalian species (Figure 4-2). The majority of these amino acid residues are either also conserved or conservatively substituted by an amino acid with similar physicochemical properties in avian, frog, zebrafish, and fruit fly species. Consistent with this data, SNAP2 (Screening for NonAcceptable Polymorphisms version 2) analysis predicted that all these point mutations would detrimentally impact protein structure (Figure 4-3). Except for p.L457P, all SIL1 
Table 4-1. MSS-associated SIL1 mutations that alter less than 6 amino acids.

\begin{tabular}{ccccc}
\hline Exon & Nucleotide change & $\begin{array}{c}\text { Amino acid } \\
\text { change }\end{array}$ & $\begin{array}{c}\text { Change in protein } \\
\text { levels / aggregation }\end{array}$ & Reference \\
\hline 4 & c.274C $>$ T & p.R92W & NA & $(173)$ \\
6 & c.617_618TC $>$ AA & p.L206Q & NA & $(174)$ \\
7 & c.691_696del & p.V231_I232del & $\downarrow$ & $(78)$ \\
9 & c.892C $>$ T & p. R298C & No change or $\uparrow$ & $(115)$ \\
9 & c.935G $>$ A & p.G312E & NA & $(99)$ \\
9 & c.934G $>$ A & p.G312R & $\downarrow$ & $(78)$ \\
10 & c. $1230 \_1244$ del & p.411_415del & $\uparrow$ & $(78,90)$ \\
10 & c. $1367 \mathrm{~T}>$ A & p.Leu456X & NA & $(88)$ \\
10 & c. $1370 \mathrm{~T}>$ C & p.Leu457Pro & NA / aggregation & $(88)$ \\
\hline
\end{tabular}

NA - Information not available.

NOTES: The c.617_618TC>AA mutation affecting leucine at position 206 is incorrectly annotated as a leucine $\rightarrow$ glutamic acid substitution at position 206 (174). In fact, the TC $>$ AA substitution in the wild-type SIL1 mRNA sequence (NM_001037633.1) causes a leucine $\rightarrow$ glutamine substitution at position 206 in SIL1's protein sequence (NP_001032722.1). 


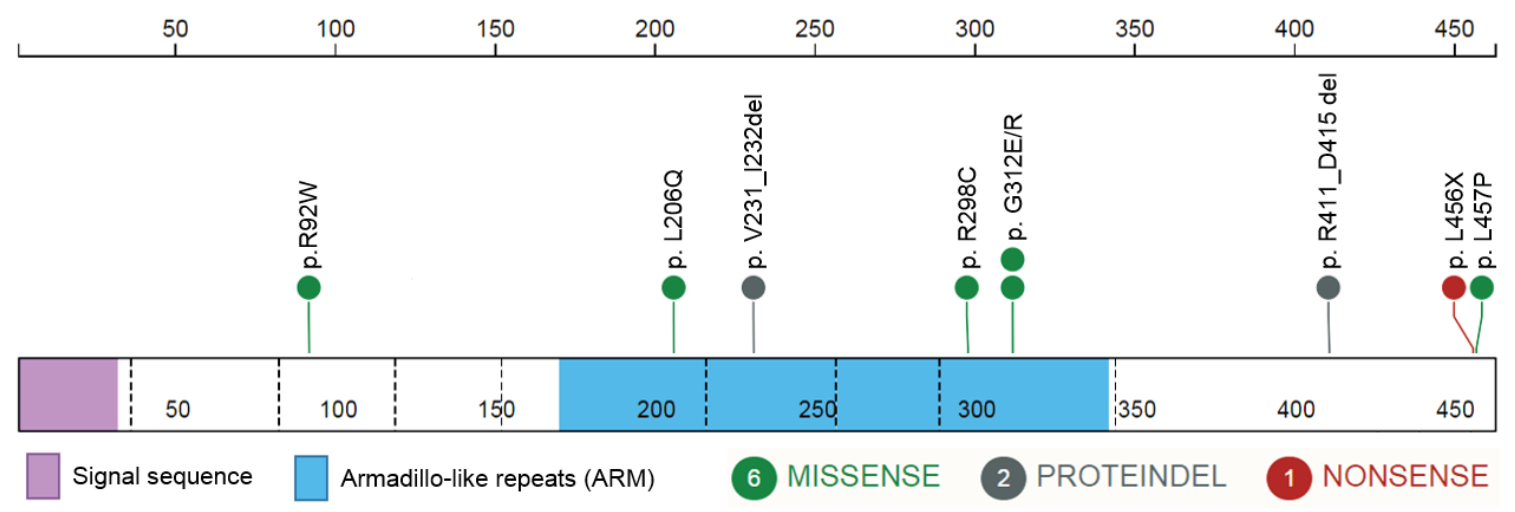

Figure 4-1. Schematic of the human SIL1 protein indicating the known MSSassociated missense mutations and small in-frame deletions.

The nine mutations include six missense mutations (green), which cause a single amino acid substitution, two in-frame deletions of either two or five amino acids, respectively (gray), and one C-terminal premature truncation of six amino acids (red). The purple segment indicates the signal sequence, the blue segment indicates the ARM-domains, and the dotted vertical lines represent exon boundaries, beginning with exon 2 since exon 1 consists of a 5' untranslated region. 
A

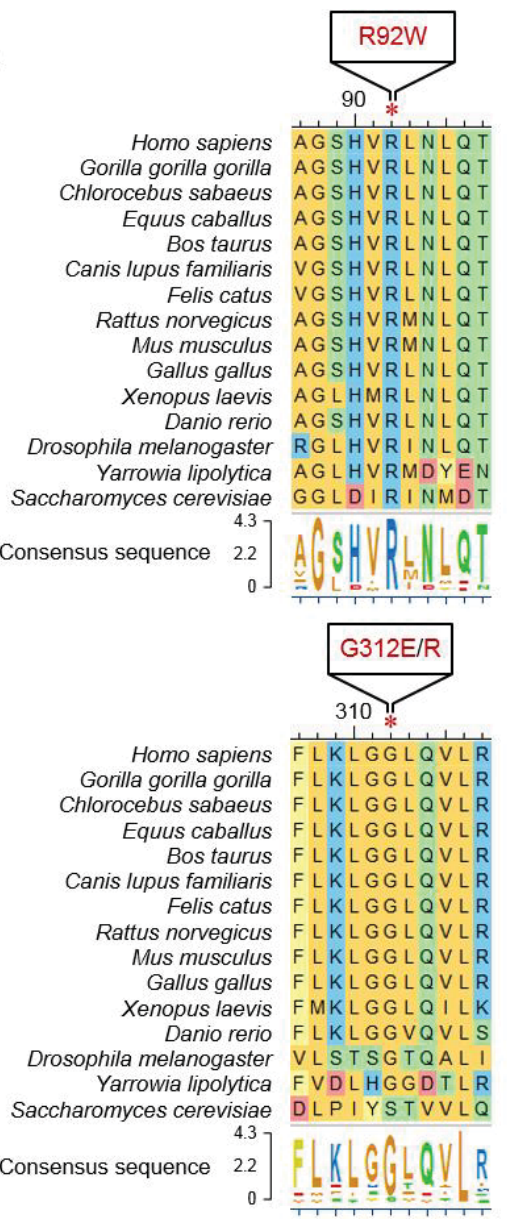

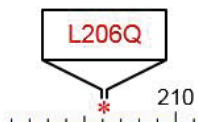

EKIAALFDLEY EK I AALFDLEY EK I AALFDLEY EK I AALFDLEY EK I AALFDLEY EK I AALFDLEY EK I AALFDLEY EKVAALFDLEY EKVAALFDLEY EKVAALYDLEY EKVSALYDLEY EKVAALLDLEY SKLDCLENLEY MLPIALSQLEE

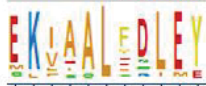

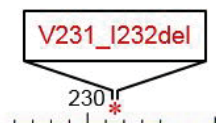

GGLQVVING . . LNS GGLQVVING - - LNS GGLQVVING. - LNS GGLQVVING - - LNS GGLQVVING.. LNS GGLQVVING -. LNS GGLQVVING - . LNS GGLQVVING -. LNS GGLQVVING - . LNS GGLRLVIEG - . LNS GALQLLINS - L LNS GGMSLVIRS -. LNS GGLDDVLLP IVVND DPLNALLQ I LEDAK - EFALLANLSLNEN
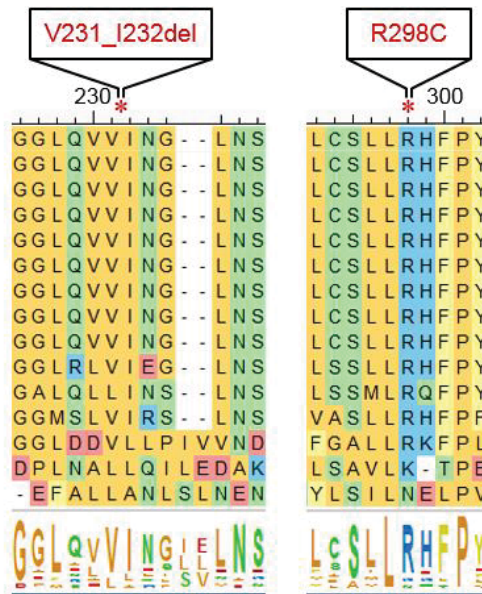

LCSLLRHFPYA LCSLLRHFPYA LCSLLRHFPYA LCSLLRHFPYA LCSLLRHFPYA LCSLLRHFPYA LCSLLRHFPYA LCSLLRHF LCSLLRHFPYA LCSLLRHFPYA LSSLLRHFPYA LSSMLRQFPYA VASLLRHFPFA FGALLRKFPLA FGALLRKFPLA \begin{tabular}{l} 
LSAVLK-TPET \\
YLS I LNELPVT \\
\hline
\end{tabular}

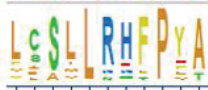

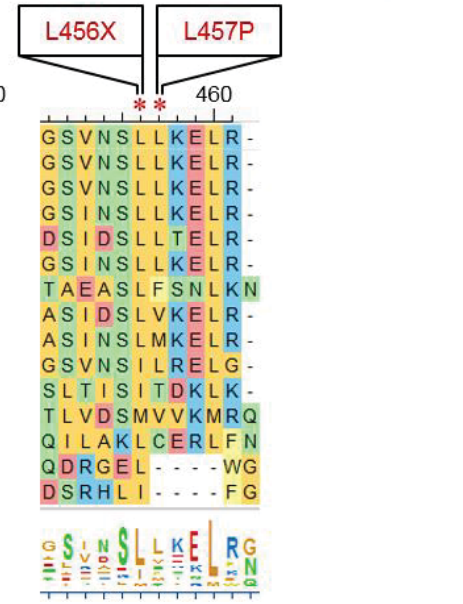

Figure 4-2. Alignment of the SIL1 protein sequence across multiple species.

(A) Alignment of SIL1 protein sequences across Homo sapiens (human), Gorilla gorilla gorilla, Chlorocebus sabaeus (monkey), Equus caballus (horse), Bos Taurus (cow), Canis lupus familiaris (dog), Felis catus (cat), Rattus norvegicus (rat), Mus musculus (mouse), Gallus gallus (chicken), Xenopus laevis (frog), Danio rerio (zebrafish), Drosophila melanogaster (fruit fly), and yeast (Yarrowia lipolytica and Saccharomyces cerevisiae) species using Clustal Omega (175). The panel below the sequence alignment depicts the consensus amino acid sequence and score across these species. The red asterisks indicate the positions of the labeled MSS-associated SIL1 mutations used in this study. 


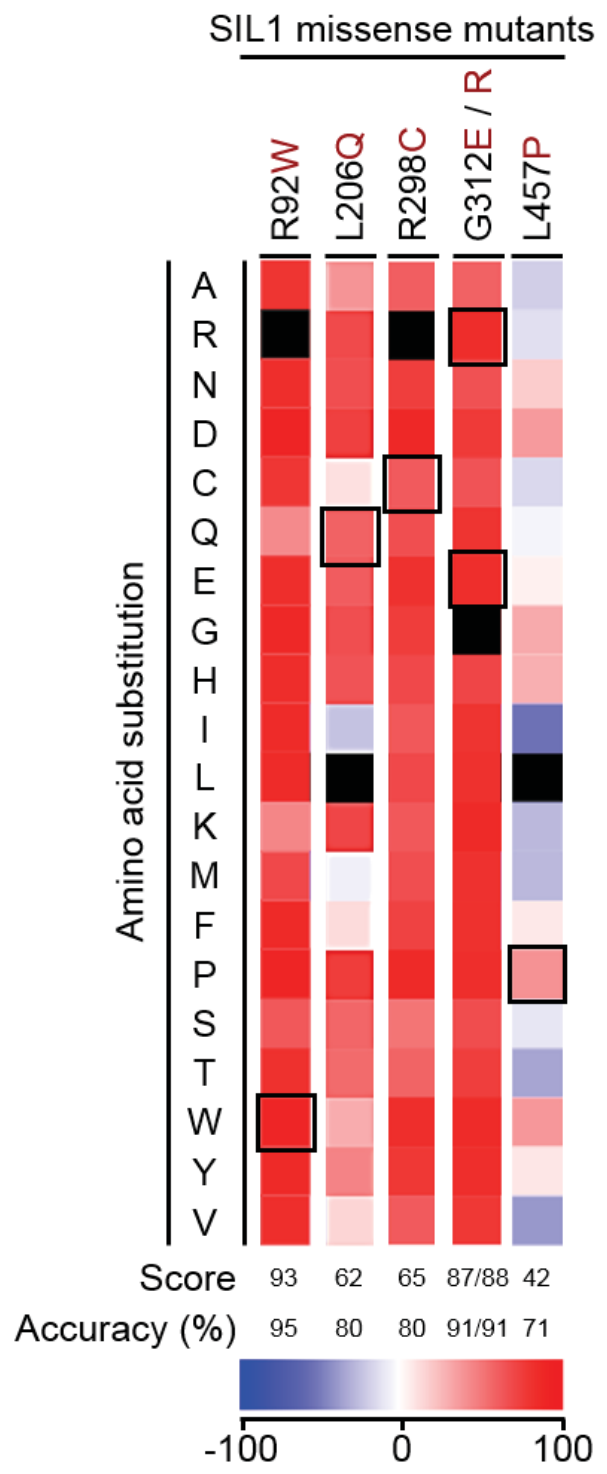

Figure 4-3. Predicted detrimental effects of point mutations in SIL1.

The potential functional effects of the six point mutations in SIL1 scores were computed using SNAP2 (176), which is based on a machine learning approach called "neural network" that uses in silico derived protein information to make predictions regarding the impact of individual substitutions on protein function. A score of $>50$ indicates a strong potential for effect (dark red), scores between -50 and 50 suggest a weaker effect (light blue to white to light red), and $<-50$ indicates a strong signal for neutral or no effect (blue). The mutant SIL1 residues are indicated as vertical panels and the amino acid substitutions across these residues are indicated on the left. The functional effect scores, the scale for these scores, and the percentage accuracy of these predictions are displayed at the bottom of the panel. Black squares in each column represent the wild-type amino acid sequence at that position. 
missense mutations displayed a high functional effect score (score $>50$ ), indicating a strong probability for detrimental effect. In the case of p.L457P, the SNAP2 prediction score was comparatively lesser (score of 42), indicating a weaker probability for the same effect.

Since SIL1 is primarily composed of alpha-helices, including the ARM domains, we used the TANGO-AGADIR algorithm to determine if the selected nine SIL1 mutations cause disruptions in the alpha-helix forming propensities of the affected regions. The arginine at position 92 in the wild-type SIL1 sequence lies upstream of a potential alpha-helix. In keeping with this, the substitution of this arginine to a tryptophan in the p.R92W SIL1 mutant did not affect the alpha-helical propensity of this region (Figure 4-4A). Instead, the TANGO algorithm predicts that this missense mutation leads to a significant increase in aggregation propensity of this region (Figure 4-5). The leucine-206 resides at the end of an alpha-helical region and the substitution to glutamine led to significant decrease in the alpha-helix forming propensity in this region (Figure 4-4B). In the case of p.V231_I232del, p.R298C, p.312E, p.G312R, p.R411 D415del, p.L456X, and p.L457P, each of these mutations led to dramatic disruptions in the alpha-helix forming propensities of the affected areas (Figure 4-4C-H).

\section{Structural modeling suggests that the selected MSS-associated mutations affect residues which play a key role in maintaining SIL1's structural integrity}

Human SIL1 shares $20.60 \%$ sequence identity with $S$. cerevisiae Sillp and $22.89 \%$ sequence identity with human HSPBP1, whereas $S$. cerevisiae Sillp is $19.75 \%$ identical to human HSPBP1 (computed using Clustal Omega). Importantly, SIL1 regions affected by MSS mutations are largely conserved with at least one other NEF, if not with both Sillp and HSPBP1. In the majority of cases, these residues are either completely conserved or occupied by similar amino acids in HSPBP1. As structural data are available for both Sil1p and HSPBP1 with the nucleotide binding domains of their corresponding Hsp70s, we reasoned that these structures could be used to understand with a reasonable degree of confidence how MSS mutations might impact their local environment within the SIL1 protein. Although the overall sequence identity between full length yeast Sillp and human HSPBP1 is quite poor, these proteins share significant structural similarities, including similar HSP70-interacting interfaces (tertiary fold-based pair-wise structure alignments computed using the jFATCAT-flexible tool yield a Pvalue of 2.50E-09) (Figure 4-6). Structural data are available for six out of the selected nine SIL1 mutated residues.

The MSS-associated SIL1 mutation p.L206Q, which corresponds to phenylalanine-155 in S. cerevisiae Sillp (Sc Sillp) and leucine-124 in human HSPBP1 (Hu_HSPBP1) sequences (Table 4-2). Both these amino acids reside within a hydrophobic pocket and are involved in hydrophobic interactions between neighboring alpha-helices (Figure 4-7 upper panel). Thus, the introduction of a polar glutamine side chain in the p.L206Q MSS mutant is likely to disrupt the hydrophobic environment and affect the packing of local alpha-helical units. The deleted two amino acids in the 
A

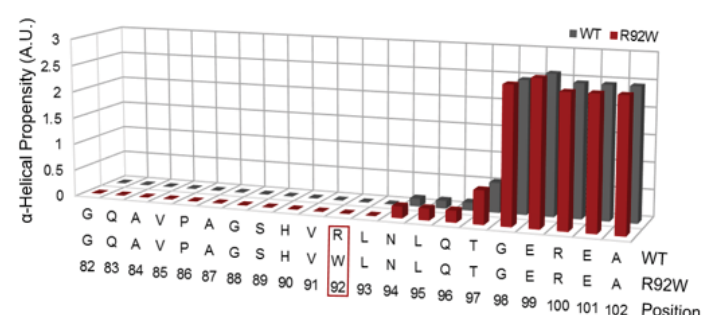

C

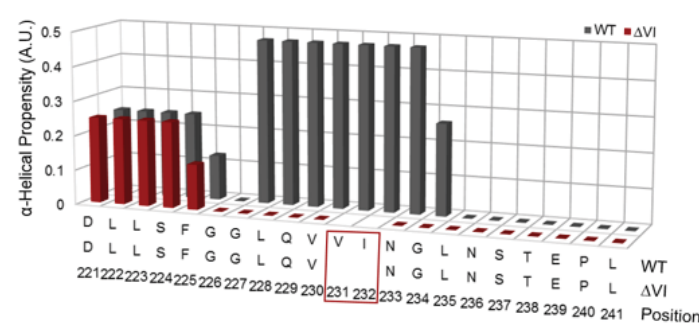

$E$

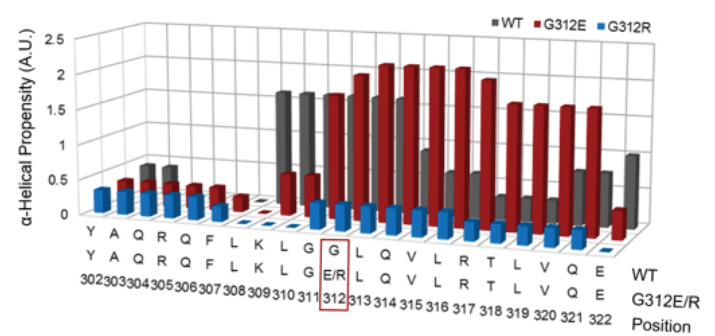

G

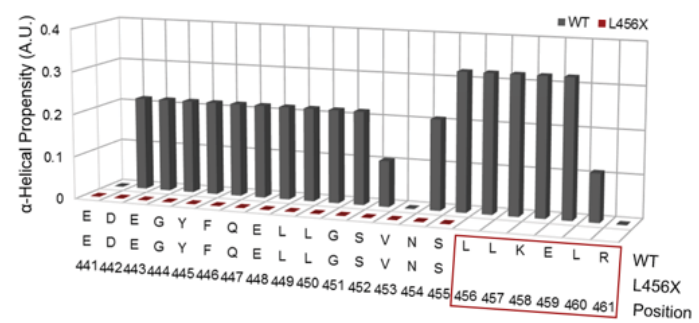

B

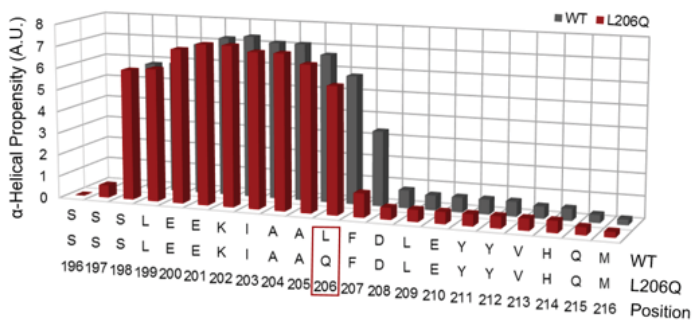

$\mathrm{D}$

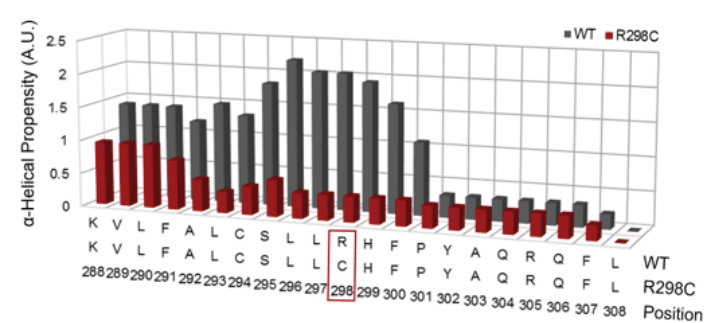

$\mathrm{F}$

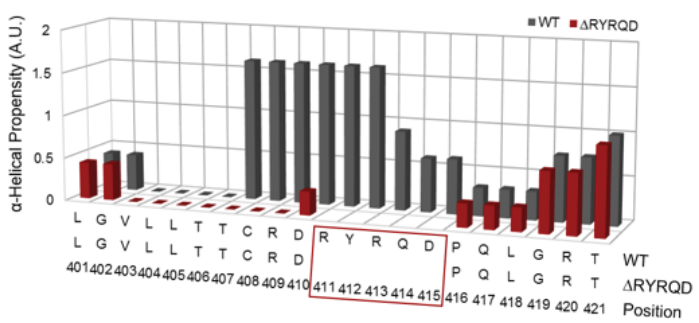

$\mathrm{H}$

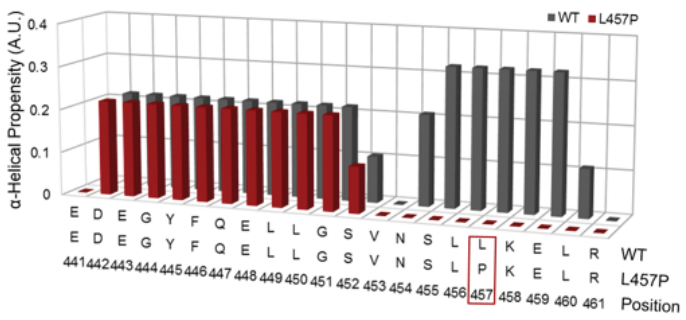

Figure 4-4. TANGO-AGADIR predicted alpha-helix forming propensities of the selected SIL1 mutant.

(A-H) Graphical representation of the alpha-helical propensities of the regions affected by p.R92W (A), p.L206Q (B), p.V231_I232del (C), p.R298C (D), p.G312E, p.G312R (E), p.R411_D415del (F), p.L456X (G), and p.L457P (H), as predicted by the TANGOAGADIR algorithm (177). Gray bars indicate the numerical values for alpha-helical propensities (expressed as Arbitrary Units, A.U.) for the wild-type SIL1 sequence (WT), whereas red bars indicate the respective values for the SIL1 mutant. The wild-type and the mutant SIL1 sequences and the position of each residue are displayed below the graphs, and the respective mutations are highlighted by a red box. 


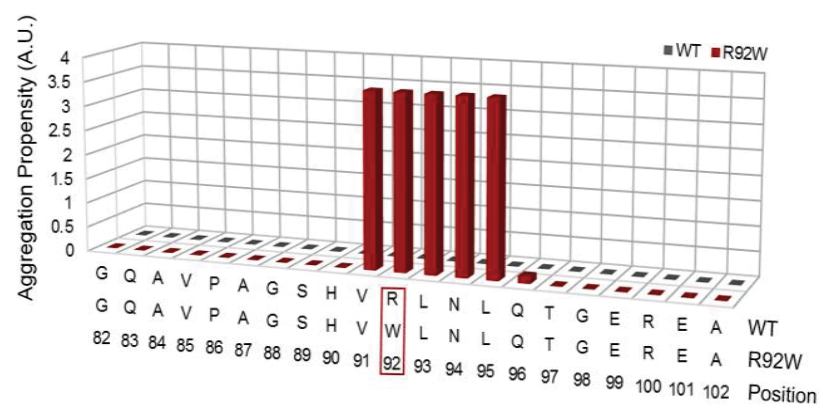

Figure 4-5. TANGO-predicted aggregation propensity of the region affected by the p.R92W point mutation in SIL1.

Graphical representation of the aggregation propensities of the region affected by p.R92W, as predicted by the TANGO algorithm (177). Gray bars indicate the numerical values for aggregation propensity (expressed as Arbitrary Units, A.U.) for the wild-type SIL1 sequence (WT), whereas red bars indicate the respective values for the SIL1 mutant. The wild-type and the mutant SIL1 sequences and the position of each residue are displayed below the graphs. The p. $\mathrm{R} 92 \mathrm{~W}$ mutation is highlighted by a red box. 


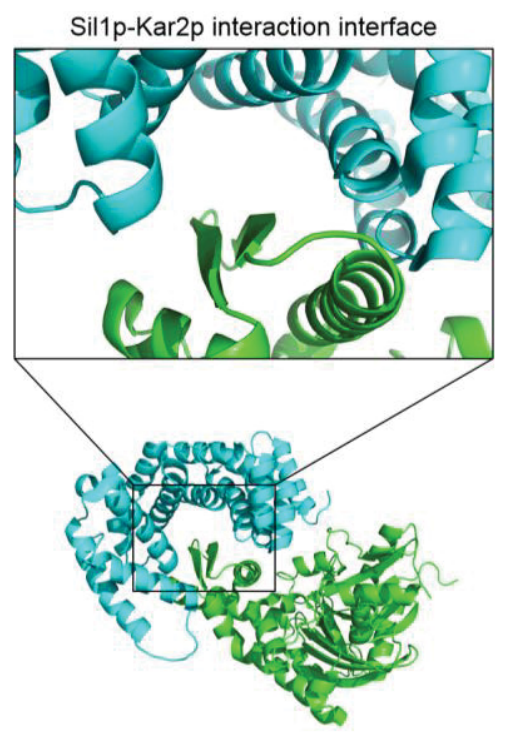

Sil1p-Kar2p NBD

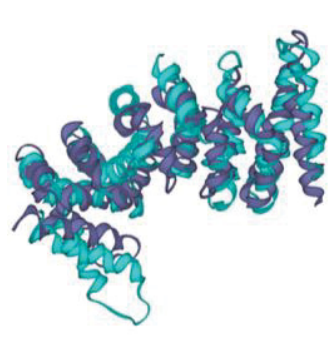

$\mathrm{RMSD}=3.569 \AA$

$\square$ Sc_Sil1p

Hu_HSPBP1

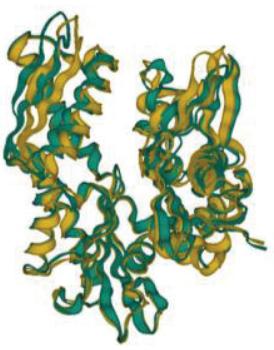

$\operatorname{RMSD}=1.634 \AA$

- Sc_Kar2p

$\square$ Hu_BiP

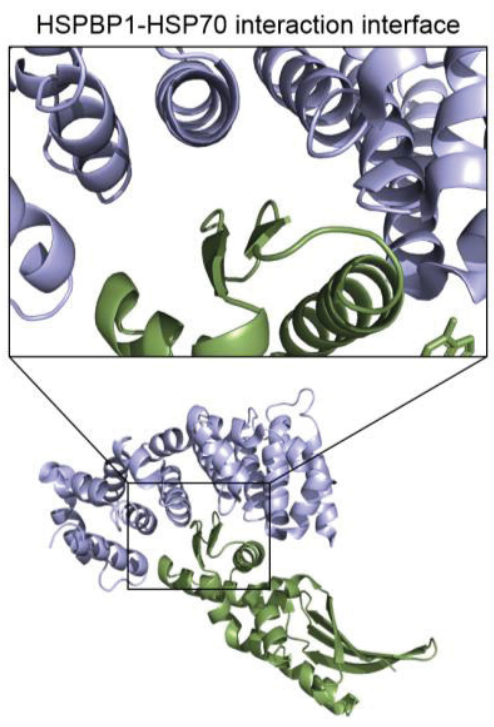

HSPBP1-HSP70 NBD

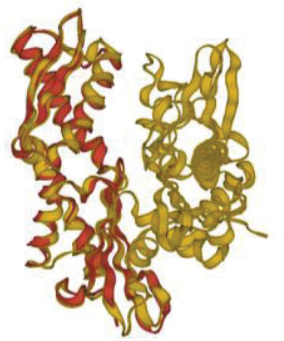

RMSD $=0.778 \AA$

Hu_HSP70

Hu_BiP

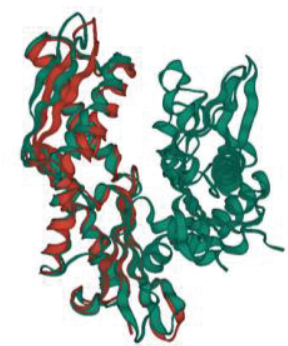

$\mathrm{RMSD}=1.076 \AA$

Hu_HSP70

Sc_Kar2p

Figure 4-6. The interaction of Sil1p and HSPBP1 with their respective HSP70 chaperones.

(Upper left panel) The structure of $S$. cerevisiae Sillp (cyan) in complex with the nucleotide binding domain of Kar2p (green) indicates that Sil1p engages lobe IIb of Kar2p, and has a minor interaction with lobe Ib (PDB: 3QML) (45). The upper panel displays a higher magnification of the Sillp-Kar2p interaction interface. (Upper right panel) The structure of human HSPBP1 (lilac) with lobe IIb of the human HSP70 nucleotide binding domain (dark green; PDB: 1XQS) $(33,172)$. The upper panel displays a higher magnification of the HSPBP1-HSP70 interaction interface. (Lower panel) Pairwise structural alignments of either Sc_Sil1p (3QML_C; cyan) with Hu_HSPBP1 (1XQS_A; lilac), or Sc_Kar2p (3QML_A; green) with either Hu_HSP70 (1XQS_D; red) or Hu_BiP (5EVZ_A; yellow) computed using the jFATCAT-rigid tool. Please note that structural data for domain I of HSP70's NBD is not available. The respective RMSD $(\AA)$ values for each structural alignment is noted under the aligned structure. RMSD, root mean square deviation. 
Table 4-2. Sil1p and HSPBP1 amino acid residues corresponding to MSSassociated human SIL1 mutants.

\begin{tabular}{ccc}
\hline Hu_SIL1 amino acid & Sc_Sil1p amino acid & Hu_HSPBP1 amino acid \\
\hline p.R92W & NA & NA \\
p.L206Q & F155 & L124 \\
p.V231_I232del & L179_A180 & L149_V150 \\
p.R298C & Not conserved & R217 \\
p.G312E & S265 & G231 \\
p.G312R & S265 & G231 \\
p.R411_D415del & K374_S378 (KARQS) & R322_Q326 (RHRCQ) \\
(RYRQD)* & NA & NA \\
p.Leu456X & NA & NA \\
p.Leu457Pro & &
\end{tabular}

NA - Structural data not available.

*Clustal Omega predicts that the sequence TTCRD-RYRQD in human SIL1 aligns with QQCKA-RQSNx in yeast Sillp. However, the structural alignment of Sillp (PDB ID 3QML) and HSPBP1 (PDB ID 1XQS) suggests that the RYRQD motif in Hu_SIL1 more accurately corresponds to the KARQS motif (amino acid residues 374-378) in Sc_Sillp. This motif superimposes on the RHRCQ motif in Hu_HSPBP1 (amino acid residues 322326), with the central arginine being conserved between all three species. 

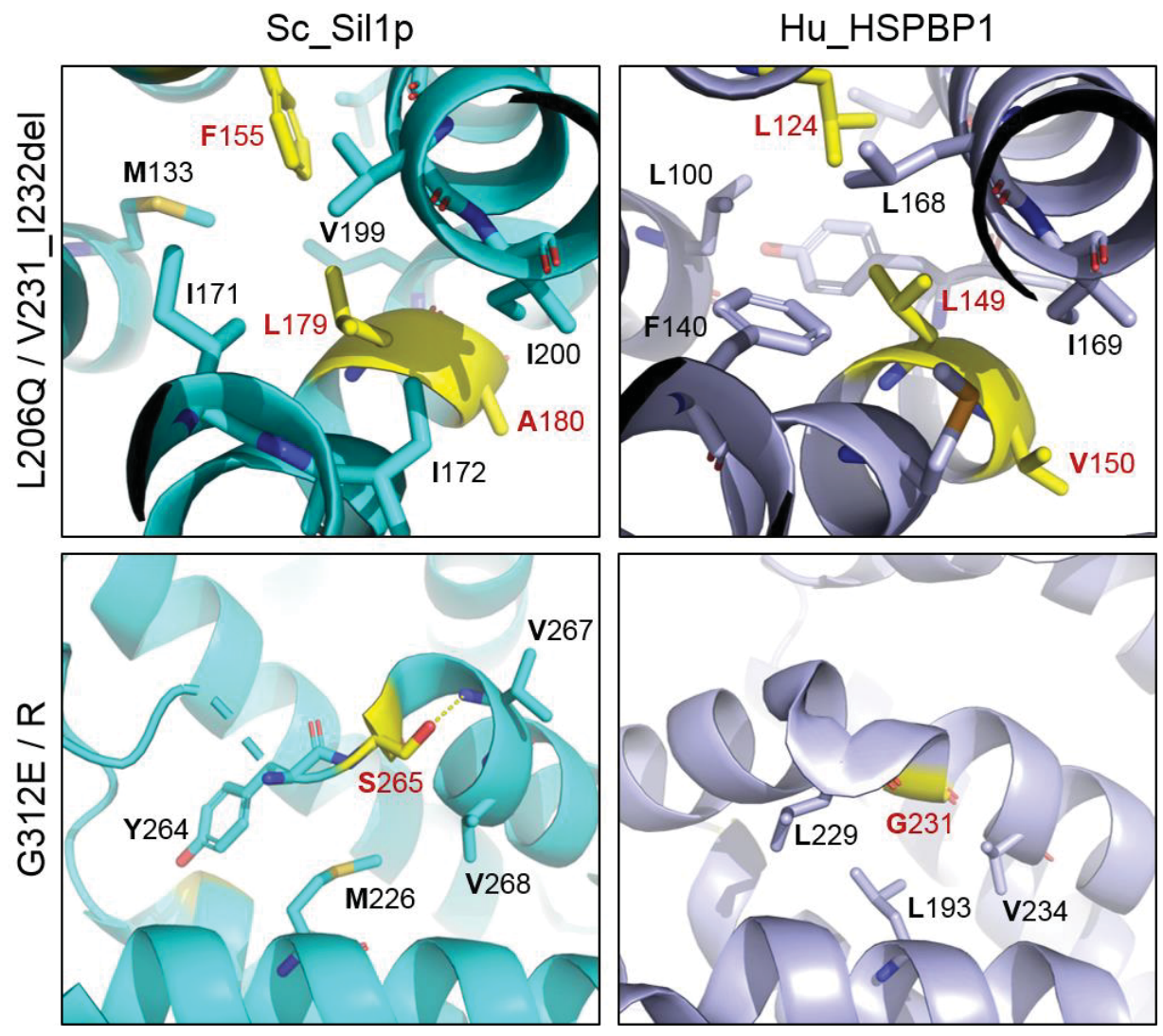

Figure 4-7. Structural mapping of the residues corresponding to p.L206Q, p.V231_I232del, and p.G312E/R.

The upper panel demonstrates the position of the corresponding F155 and L179-A180 residues in Sc_Sillp (cyan, left) and L124 and L149-V150 in Hu_HSPBP1 (lilac, right), which are affected by the p.L206Q and p.V231_I232del mutations (yellow, label in red), respectively. The residues constituting the hydrophobic core are labeled in black. The lower panel similarly demonstrates the location of the corresponding S265 in Sc_Sillp (cyan, left) and G231 in Hu_HSPBP1 (lilac, right), which are affected by the p.G312E and p.G312R mutations (yellow, label in red), respectively. The amino acid residues constituting the hydrophobic stretch juxtaposed to S265 and G231 are labeled in black. 
p.V231_I232del SIL1 mutant correspond to similar hydrophobic residues, leucine-179 and alanine-180 in Sc_Sil1p, and leucine-149 and valine-150 in $\mathrm{Hu}$ HSPBP1, respectively. These amino acids are also embedded within the same hydrophobic pocket as phenylalanine-155 and leucine-124 (Figure 4-7 lower panel), and the deletion of these amino acids might directly affect the stability of this hydrophobic core. In addition, this deletion would be predicted to insert the subsequent polar amino acid, asparagine-181 into the hydrophobic core in Sc_Sillp, which may not be tolerated. This asparagine is only conserved between yeast and human SIL1 (asparagine-233).

Serine-265 in Sc_Sil1p and glycine-231 in Hu_HSPBP1 correspond to glycine312 in human SIL1, which is mutated to either an arginine or a glutamine (p.G312E/R) in a subset of American and Dutch MSS patients. These residues are juxtaposed to a stretch of hydrophobic residues, constituting of tyrosine-264, methionine-226, valine-268, and valine-267 in Sc_Sillp, and leucines at positions 229, 193, and 234 in Hu_HSPBP1 (Figure 4-7). Both serine-265 and glycine-231 are relatively small in size and offer increased conformational flexibility, especially in tight turns, as exemplified by the glycine-231 in Hu_HSPBP1. The substitution of these residues by either an arginine, in the case of p.G312R, or glutamic acid, in the case of p.G312E, can have disastrous consequences for two reasons. Both, arginine and glutamic acid, have charged sidechains with relatively larger geometries compared to glycine. Thus, these large polar substitutions are likely to create steric hindrances that disrupt interactions among the neighboring alpha-helices in the hydrophobic pocket.

Although the MSS-associated p.L206Q and p.G312E/R mutations reside within exons 6 and 9, respectively, both of which are predicted to be major BiP interactions sites, these mutations are likely to disrupt intramolecular interactions and destabilize SIL1 rather than affecting its BiP-interaction motif. The p.R298C and p.R411_D415del mutations correspond to regions in Sc_Sillp and Hu_HSPBP1 that interact with their respective HSP70 chaperones. Arginine-298 in SIL1 corresponds to arginine-217 in Hu_HSPBP1, while Sc_Sillp uses a different sequence, albeit with a similar structural interface to interact with Kar2p. Positively charged, polar arginine-217 lies at the epicenter of a hydrogen bond network. This arginine forms hydrogen bonds with HSP70 glutamic acid-283, as well as with the mainchain and sidechain of serine-281 in HSP70, thereby contributing to one of the major HSP70-binding interfaces of HSPBP1. In addition, Hu_HSPBP1 glutamine-174, serine-213, and asparagine-256 sidechains form intramolecular hydrogen bonds with the arginine-217 sidechain, thus, stabilizing the conformation of the HSP70-interacting groove, which also includes neighboring helices (172) (Figure 4-8 upper panel). Thus, the substitution of arginine-298 with the shorter and uncharged cysteine-sidechain in p.R298C could be potentially destabilizing the HSP70-binding interface of HSPBP1, in keeping with decreased alpha-helical propensity predicted by TANGO-AGADIR, and could lead to decreased binding affinity for HSP70. In addition, the introduction of a reactive cysteine residue adjacent to another cysteine (i.e. cysteine-294 in Hu_SIL1 and cysteine-214 in Hu_HSPBP1) may also introduce an aberrant disulfide bond, which can further potentiate the negative consequences of this substitution. 

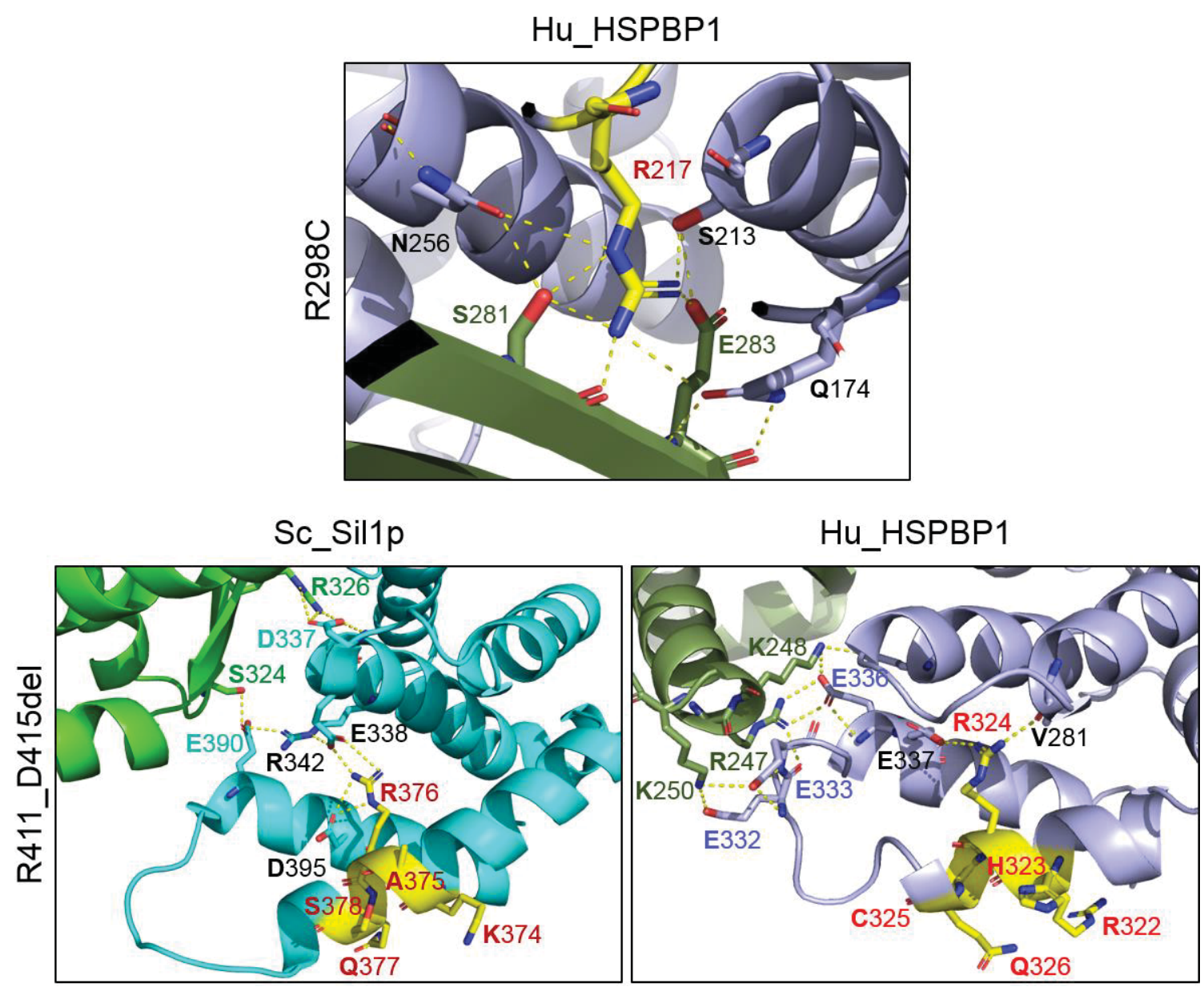

Figure 4-8. Structural mapping of the residues corresponding to p.R298C and $\triangle R Y R Q D$.

The upper panel demonstrates the position of the corresponding R217 in Hu_HSPBP1 (lilac), which is affected by the p.R298C mutation (yellow, label in red). The structure of HSP70 NBD and the HSP70 residues, which forms hydrogen bonds with HSPBP1 (S281 and E283), are displayed in dark green. Hydrogen bonds are illustrated as yellow dashed lines. Neighboring HSPBP1 amino acids forming hydrogen bonds with R217 are labeled in black. The lower panel demonstrates the positions of K374-S378 amino acids in Sc_Sillp (cyan) and R322-Q326 in Hu_HSPBP1 (lilac), which correspond to amino acids affected by the $\triangle$ RYRQD mutation in human SIL1 (yellow, label in red). The structure of the nucleotide binding domains of Kar2p and HSP70, and their residues which forms hydrogen bonds with either Sc_Sillp or Hu_HSPBP1 are displayed in bright and dark green, respectively. The residues on Sc_Sillp interacting with Kar2p are labeled in cyan, and the residues on Hu_HSPBP1 interacting with HSP70 are labeled in purple, respectively. Hydrogen bonds are illustrated as yellow dashed lines. Neighboring Sc_Sillp and Hu_HSPBP1 amino acids forming hydrogen bonds are labeled in black. 
The MSS-associated p.R411_D415del SIL1 mutation leads to an in-frame deletion of five amino acids (RYRQD). The central arginine-413 of the deleted RYRQD motif in human SIL1 corresponds to arginine-376 in Sc_Sil1p and arginine-324 in $\mathrm{Hu}$ HSPBP1. Although this conserved arginine does not directly interact with Kar2p or HSP70, it seemingly plays a role in intramolecular stability of the Sillp-Kar2p and HSPBP1-HSP70 interaction sites (Figure 4-8 lower panel). In Sc_Sillp, arginine-376 forms polar interactions with glutamic acid-338 and aspartate-395 of neighboring alphahelices, which additionally harbor multiple residues that directly interact with Kar2p, such as glutamic acid-390 and aspartate-337 that form hydrogen bonds with serine-324 and arginine-326 of Kar2p, respectively. Consistent with our interpretation, mutations of the Sc_Sil1p glutamic acid-390 and aspartate-337 residues to alanine have been reported to significantly decrease the affinity of Sillp for Kar2p (45). Arginine-324 in $\mathrm{Hu}$ _HSPBP1 also adopts a crucial role in stabilizing the intramolecular topology of neighboring $\mathrm{C}$-terminal helices by forming inter-chain hydrogen bonds with the glutamic acid-337 sidechain and the valine-281 mainchain of Hu_HSPBP1 (Figure 4-8 lower panel). These reside near numerous polar interactions between the glutamic acid residues at positions 290, 332, 333, and 336 in Hu_HSPBP1 with the HSP70 lysines at positions 248 and 250, and arginine-247. Thus, the loss of this conserved arginine can potentially destabilize Sc_Sil1p's and Hu_HSPBP1's BiP-interaction site.

\section{MSS-associated SIL1 mutants form NP40-insoluble aggregates}

It has been argued that alpha helices are more robust at withstanding mutations than beta-strands due to a greater number of inter-residue contacts in helices (178). Alpha-helical predictions by TANGO and our structural modeling indicate that eight of these nine SIL1 mutations are likely to disrupt regional alpha helices and modify SIL1's structure, whereas the remaining p.R92W mutation increases its aggregation propensity. Thus, we examined the biochemical consequences of these mutations by exogenously expressing them in COS-1 cells. To determine if these SIL1 mutants aggregated, we analyzed the NP40-soluble and -insoluble fraction of cell lysates by western blotting. Surprisingly, all mutants displayed a tendency to aggregate, as evidenced by the accumulation of SIL1 mutants in the NP40-insoluble fraction (Figure 4-9A, B). Although we previously reported that the p.L457P mutant, which affects the very Cterminus of SIL1, aggregates (88), these data reveal it is comparatively less aggregationprone than several other mutants.

To further explore the aggregation propensity and behavior of these SIL1 variants, we treated COS-1 cells expressing wild-type and mutant SIL1 with a membrane permeable, reversible cross-linker, dithiobis succinimidyl propionate (DSP), which has a thiol cleavable disulfide bond. We processed the NP40-soluble lysates for western blotting under non-reducing and reducing conditions. For all SIL1 mutants, we observed higher molecular weight species under non-reducing conditions that range from approximately $110 \mathrm{kDa}$ to greater than $260 \mathrm{kDa}$, whereas wild-type migrates as a $56 \mathrm{kDa}$ band (Figure 4-9C). In all cases, when the cross-linked samples were treated with a 


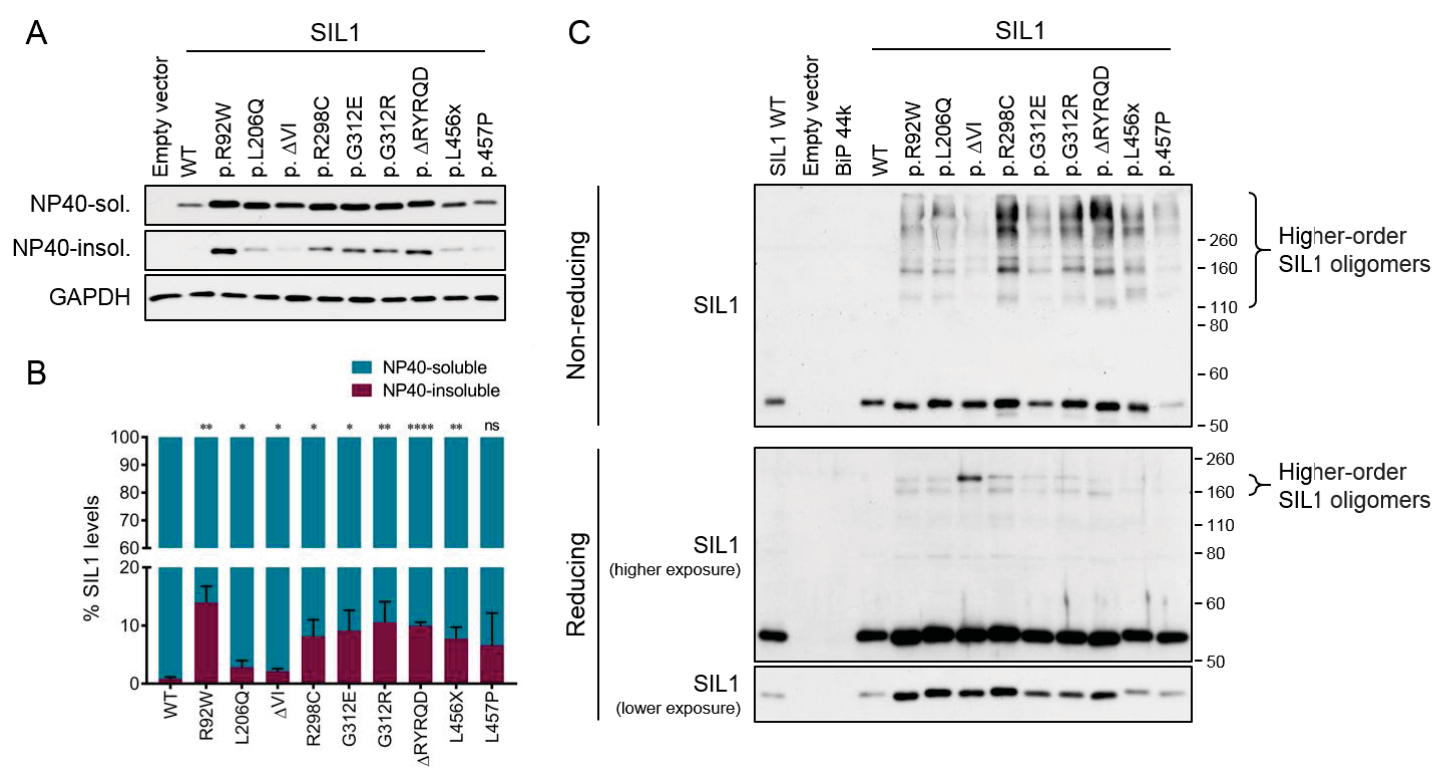

Figure 4-9. MSS-associated SIL1 mutants form NP40-insoluble aggregates.

(A) COS-1 cells were transfected with either empty pSVL vector, or pSVL containing wild-type SIL1 or mutant SIL1 cDNA. After 24 hours, these cells were lysed with NP40 lysis buffer and the NP40-soluble and -insoluble fractions were collected and processed for western blotting under reducing conditions and probed for SIL1. The western blots displayed are representative of three experiments. GAPDH serves as a loading control. (B) Graph represents densitometric quantitation of western blotting data $(n=3)$ from (A) for the NP40-soluble (blue) and NP40-insoluble (red) fractions. GAPDH-normalized, NP40-soluble plus -insoluble SIL1 levels were designated as total SIL1 and expressed as $100 \%$, whereas the NP40-soluble and -insoluble levels are displayed as a fraction of total SIL1. Error bars indicate means \pm s.e.m. (C) COS-1 cells were either transfected with

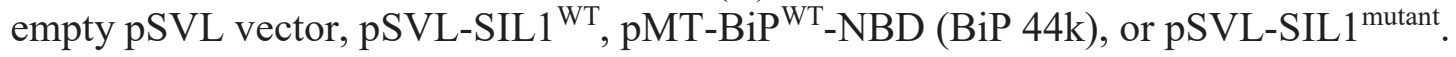
After 24 hours, these cells were crosslinked using DSP and lysed using RIPA lysis buffer. The RIPA-soluble lysates were processed for western blotting under non-reducing (upper blot) and reducing (lower blots) conditions and probed with SIL1. Western blotting results are from one experiment $(n=1)$. Two exposures for lysates processed under reducing conditions are displayed. The higher molecular SIL1 oligomers in the nonreducing and reducing blots are indicated on the right. 
reducing agent prior to SDS-polyacrylamide electrophoresis, the larger species were primarily resolved into a single $55 \mathrm{kDa}$ band. Thus, the slower migrating species present in the SIL1 mutants represent NP40-soluble SIL1 oligomers and are a clear indication of misfolding.

\section{MSS-associated SIL1 mutants alter its half-life}

We consistently observed higher steady-state expression of several SIL1 mutants. While misfolded proteins are often retrotranslocated to the cytosol for degradation via the ubiquitin-proteasomal system, the formation of aggregates can interfere with their degradation. To determine if this was indeed the case, we transfected COS-1 cells with wild-type SIL1 and the nine mutants, performed a cycloheximide ( $\mathrm{CHX}$ ) chase, and analyzed the levels of SIL1 in the NP40-soluble fraction by western blotting. The halflife of wild-type SIL1 was determined to be approximately three hours, in accord with our previous result (88). Interestingly, we observed that a subset of SIL1 mutants displayed increased stability over a six-hour timeframe with minimal degradation, whereas the remaining SIL1 mutants displayed increased turnover and degradation kinetics, relative to wild-type levels (Figure 4-10). The subset of SIL1 mutants displaying increased protein stability consisted of p.R92W, p.L206Q, p.R298C, p.G312E, p.G312R, and $\triangle \mathrm{RYRQD}$, whereas $\triangle \mathrm{VI}$, p.L456X, and p.L457P mutants displayed fasterturnover compared to wild-type SIL1. With the exception of p.L206Q, these mutants distinctly segregate into the two classes based on their aggregation tendencies.

\section{Discussion and Future Direction}

This is currently an ongoing project within the lab and is pending completion. Thus far, we show that the selected nine MSS-associated SIL1 point mutations affect residues that are highly conserved in mammals. Bioinformatic analyses suggested that these mutations are likely to have detrimental effects on SIL1 structure. The large majority of the affected residues reside within alpha-helices, which are likely to be disrupted by the MSS-associated pathogenic substitutions. Structural data based on yeast Sillp and human HSPBP1, the cytosolic ortholog of SIL1, are consistent with these indications and further argue that the introduction of mutant residues are likely to perturb intramolecular interactions required for achieving a native structure. In addition to affecting intramolecular interactions, two of the mutations, p.R298C and p.R411_D415del, affect amino acids that reside in the vicinity of a major and minor BiPinteraction interface, respectively, and thus are also likely to affect the ability of SIL1 to interact with BiP. All nine SIL1 mutants were aggregation prone, albeit with varying characteristics and propensities, thus, confirming that the selected MSS-associated SIL1 mutations affect the folding properties of SIL1. We additionally observed a distinction in the half-lives of these mutants. A subset of SIL1 mutants, including p.R92W, p.G312E, p.G312R, and p.R411_D415del mutants, displayed significantly increased stability, whereas the p.V231_I232del, p.L456X, and p.L457P mutants were rapidly cleared by the cell. These differences may at least partially stem from their tendency to aggregate, 
A

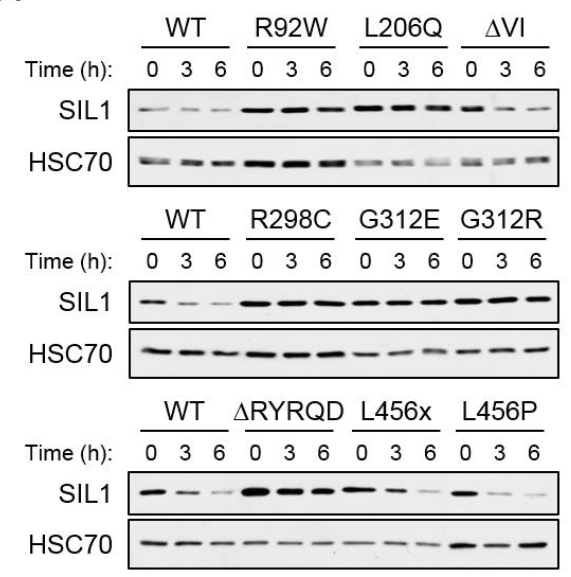

B

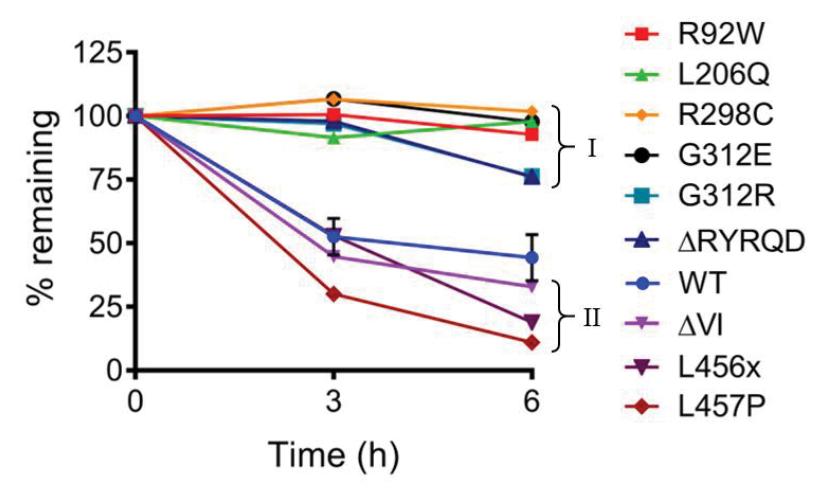

Figure 4-10. SIL1 mutations alter its stability and degradation kinetics.

(A) COS-1 cells were transfected with the pSVL vector containing either wild-type (WT) or mutant SIL1. 24 hours later, these cells were treated with the protein synthesis inhibitor, $\mathrm{CHX}$, and chased for the indicated timeframes. These cells were then lysed with NP40 lysis buffer and the NP40-soluble fraction was processed for western blotting for SIL1. (B) Graphical representation of the percentage of SIL1 remaining at the indicated timepoints. These values are derived from densitometric quantitation of the half-lives of WT $(n=3)$ and SIL1 mutants $(n=1)$ from (A), normalized to $100 \%$ at the zero timepoint. HSC70 serves as a control for equivalent loading. The error bars for WT indicate means \pm s.e.m. 
displayed a relatively lower tendency to aggregate, which is in keeping with the fact that large protein aggregates are more difficult to retrotranslocate and degrade.

\section{Completing studies on the half-lives of SIL1 mutants}

These results indicate that the latter subset of SIL1 mutants may be effectively recognized as targets of the ER-associated degradation (ERAD) machinery, which allow rapid clearance, whereas the highly-aggregation prone SIL1 mutants may predominantly depend on autophagy for the degradation of high molecular weight aggregates. This is partially supported by our previously published data for the p.L457P mutant, which demonstrates that the treatment of cells expressing this mutant with the proteasomal inhibitor, MG132, resulted in stabilization of this mutant, whereas the disruption of autophagy in these cells by ammonium chloride treatment did not have a significant effect (88). In addition to achieving at least three experimental trials for the CHX-chase experiments, it is important to confirm if the two classes of SIL1 mutants indeed segregate into these classes according to their heir half-lives.

\section{Do the selected SIL1 mutants display a dominant-negative behavior?}

In keeping with our data, the SIL1 mutations used in this study affect its folding properties and cause aggregation. It is possible that SIL1 misfolding, perhaps for a subset of these MSS-associated mutants, may cause a dominant-negative effect by which the mutant SIL1 protein may antagonize the function of wild-type SIL1 by co-aggregation. This mechanism has been well exemplified in the case of epidermolysis bullosa simplex, involving dominant-negative mutations in keratin 5 and keratin 14, and by a subset of mutations affecting p53 in cancer (179). Engineering an N-terminal tag after the ERtargeting signal sequence for wild-type SIL1 that adds sufficient molecular weight to distinguish the tagged wild-type and untagged mutant SIL1 proteins by SDS-PAGE would permit co-immunoprecipitation experiments. This would allow us to determine if

SIL1 mutants co-immunoprecipitate with the wild-type SIL1, and if they form multimeric species or higher molecular weight aggregates under non-reducing conditions. The introduction of an N-terminal tag compared to a C-terminal tag is preferred since the latter plays a role in maintaining SIL1's structural integrity and may introduce experimental artifacts (88).

\section{Evaluating the ability of chemical chaperones to rescue SIL1 mutants}

The primary criteria for selection of the SIL1 mutants used in this study out of the 46 reported MSS-associated mutations was that they affect less than six amino acids and retain the ARM domains, which mediate SIL1's interaction with BiP. Classically, MSS displays Medelian, autosomal recessive inheritance and heterozygous individuals carrying one allelic mutation in SIL1 do not apparently display the cardinal features of MSS. Thus, it is possible that stabilizing even small amounts of SIL1 mutants may 
partially rescue the defects associated with SIL1 loss and alleviate the severity of the MSS-associated symptoms.

Chemical chaperones are low molecular weight compounds that function similarly as molecular chaperones, with respect to their role in maintaining proteostasis and favoring the folded conformation of a protein, but have a nonspecific mode of action (180). Small molecules, sodium 4-phenylbutyrate (PBA) and tauroursodeoxycholic acid (TUDCA), are hydrophobic chemical chaperones, whose proposed mechanisms of action involves their interaction with exposed hydrophobic protein regions, which are generally buried in correctly folded protein conformations, but surface exposed in misfolded or unfolded states. Both PBA and TUDCA are orally bioavailable, blood-brain-barrier (BBB)-permeable, have a no-to-low toxicity profile, and are Food and Drug Administration (FDA)-approved for the treatment of urea cycle disorders or primary biliary cirrhosis, respectively for PBA and TUDCA (180). PBA has been effective in restoring folding of mutant forms of CFTR $(181,182), \alpha 1$-antitrypsin (183), serotonin transporter (184), uromodulin (185), and nephrin (186), which are all synthesized in the ER, and to ameliorate the aggregation of desmin intermediate filaments in an animal model of myofibrillar myopathy and primary myotubes derived from this model (187). TUDCA has proven effective to restore folding of mutant laminin $\beta 2$ (188), and to protect neurons against poly(GA)-induced ER stress and toxicity (189). To test the activity of these agents in preventing aggregation or promoting the stability of mutant SIL1 proteins that are rapidly degraded, we will transfect COS-1 cells with vectors encoding the nine SIL1 mutants and add either PBA or TUDCA 24 to 72 hours prior to assaying these mutant proteins. To determine the optimal concentration of PBA and TUDCA to use for these experiments, we will perform a dose response curve based on previously reported concentrations of these chemical chaperones, and assay SIL1 levels in the NP40-soluble and -insoluble fractions by western blotting. We will then determine the effects of PBA and TUDCA on the half-life of SIL1 mutant proteins, using a similar approach as demonstrated in Figure 4-10. Lastly, for those SIL1 mutants that are rescued by the treatment with either PBA or TUDCA, we will determine if this treatment also restores the ability of mutant SIL1 to associate with BiP at levels comparable to the wild-type protein as one method of determining if chemical chaperones may indeed restore the function of SIL1 mutants.

\section{Investigating the ability of SIL1 mutants to bind BiP}

The next critical aspect of characterizing these SIL1 mutants is to test their ability to interact with the nucleotide binding domain of BiP function, which governs the nucleotide exchange activity of SIL1. This experiment is especially crucial to understand the mechanisms by which the p.R298C and the p.R411_D415del mutations lead to a loss of SIL1's function, since both of these residues are suggested to function in maintaining the structural integrity of the BiP-interaction interface. These predictions are consistent with a previous report, which shows that both the p.R411_D415del and p.L457P SIL1 mutants immunoprecipitate lower amounts of full length BiP, relative to wild-type SIL1 or HSPBP1, and are aggregation-prone $(78,90)$. However, from a mechanistic 
standpoint, it remains unclear if BiP recognizes the mutant SIL1 proteins as partially unfolded or misfolded substrates, or if the mutant SIL1 indeed retains its ability to interact with BiP's NBD. There are two strategies to solve this conundrum. The first is co-transfecting the wild-type and mutant SIL1 along with the BiP nucleotide binding domain, which harbors either the G226D or the G227D mutation (residues correspond to hamster BiP sequence) in COS-1 cells, and immunoprecipitating with anti-BiP antibody to assay the amount of co-immunoprecipitating wild-type and mutant SIL1. It has been previously demonstrated that SIL1 preferentially binds to the ADP-bound conformation of $\operatorname{BiP}(63,190)$. The G226D and G227D BiP NBD mutations, which disrupts the ATPbinding site in each case, behave in a similar manner as the ADP-bound conformation of $\mathrm{BiP}$ and readily engage SIL1 (63). These BiP NBD mutants are also unaffected by cellular ATP, which causes disengagement of the SIL1-BiP interaction upon lysis, and allows us to assay the interaction of BiP with the various SIL1 mutants without the requirement for cross-linking. The second strategy relies on engineering select point mutations in BiP that allow us to dissect the binding of SIL1 as a NEF versus as a substrate. Two point mutations in domain IIb of Kar2p's NBD, E311A and R317, have been shown to selectively disrupt Sillp binding without altering the ability to interact with Lhs1p, the yeast GRP170 homolog (65). Conversely, the V461F and R492E mutations in BiP's SBD have been independently shown to significantly compromise its ability to interact with substrates $(191,192)$. After optimization studies to validate the full-length BiP mutants, we will co-transfect wild-type and mutant SIL1 encoding cDNAs in COS-1 cells, along with either wild-type BiP, the BiP NBD mutant, or the BiP SBD mutant, and determine the amount of SIL1 co-immunoprecipitating with each of these BiP constructs, allowing us to determine the total amount of SIL1 that binds BiP, and the amount of SIL1 that engages BiP as a NEF or as a client protein. The H214A point mutation in SIL1, corresponding to histidine-163 in Sillp, has been reported to disrupt its binding with BiP's NBD and can serve as a negative control for the interaction of SIL1 with BiP as a NEF (45).

\section{Investigating the NEF activity of corrected SIL1 mutants}

For those SIL1 mutants that are able to be successfully salvaged in terms of their aggregation propensity, stability, and their ability to interact with $\mathrm{BiP}$ upon treatment with chemical chaperones, we will then determine if these SIL1 mutants have NEF activity as the final piece in this manuscript. Since this is an in vitro assay, we will require recombinant SIL1 protein. While we were able to successfully achieve sufficient levels of wild-type SIL1 (63), the mutant SIL1 proteins are aggregation-prone when expressed in mammalian cells, they are likely to form inclusion bodies in bacteria. However, we are well equipped to circumvent this challenge and have successfully resolubilized proteins, such as ERdj3 mutants, from inclusion bodies (193). We will determine the ability of SIL1 mutants that are amenable for rescue by chemical chaperones to increase BiP's ATPase activity, which will be evaluated by both nucleotide exchange and ATPase assays, as previously performed (63). 
Overall, our results will offer mechanistic insights into how the selected MSSassociated SIL1 mutations could disrupt regional interactions and destabilize alphahelices, rendering mutant SIL1 more aggregation prone with aberrant degradation kinetics, thus, explaining how these mutations cause a loss of functional SIL1 and MSS. We will further explore two approaches using hydrophobic chemical chaperones, PBA and TUDCA, that have demonstrated promising preclinical results for various protein misfolding indications, to rescue the defects associated with these SIL mutations.

\section{Materials and Methods}

\section{Bioinformatics}

\section{Multiple sequence alignment}

The FASTA sequences for SIL1 from numerous organisms were obtained from UniProt (https://www.uniprot.org/). Their UniProt IDs are as follows: Homo sapiens (Q9H173), Gorilla gorilla gorilla (G3RAN4), Chlorocebus sabaeus (A0A0D9RKX0), Equus caballus (F6ZBH7), Bos Taurus (Q32KV6), Canis lupus familiaris (F1PLP6), Felis catus (M3VX58), Rattus norvegicus (Q6P6S4), Mus musculus (Q9EPK6), Gallus gallus (F1NWG7), Xenopus laevis (Q6NUA7), Danio rerio (A0A0G2KDW7), Drosophila melanogaster (Q9VBV5), Yarrowia lipolytica (Q99158), and Saccharomyces cerevisiae (Q08199). Multiple sequence alignment of these protein sequences, using Clustal Omega, and the phylogenetic tree, depicting the evolutionary relationships of these organisms with respect to the SIL1 protein sequence, was computed with the DNASTAR ${ }^{\circledR}$ MegAlign Pro software (DNASTAR, Inc., Madison, WI).

\section{Prediction of functional effects of missense SIL1 mutations}

The functional effects of missense SIL1 mutations were predicted in silico using the Screening for Non-Acceptable Polymorphisms software (SNAP2;

https://www.rostlab.org/services/SNAP) (176). SNAP2 is a classifier based on a machine learning device called the "neural network," which predicts the potential impact of single amino acid substitutions on protein function, and has been demonstrated to outperform SNAP, PolyPhen-2, and SIFT. The FASTA sequence for human SIL1 (UniProt ID Q9H173) was used as input. SNAP2 reports the prediction score of all possible substitutions for each amino acid within a protein and their percentage accuracy and displays the prediction scores as a heatmap. Dark red indicates a high score (score $>50$, strong signal for effect), white indicates weak signals $(-50<$ score $<50)$, and blue indicates a high negative score (score $<-50$, strong signal for neutral or no effect).

\section{Prediction of alpha-helical and aggregation propensities using TANGO}

The alpha-helix and aggregation forming propensities of the various SIL1 mutants were computed with the default parameters and conditions using TANGO 
(http://tango.crg.es/), which in turn uses AGADIR (AGADIR-1s11) with the implementation of the multiple partition function for calculating alpha-helical propensities. The complete sequence of either human wild-type or mutant SIL1 was used for input. The graphs in Figure 4-4 and Figure 4-5 only portray either the alpha-helical or aggregation propensity values for the region consisting of the mutation and ten amino acids upstream and downstream of the mutation. Graphs were plotted using Excel 2016 (Microsoft, Inc., Redmond, WA).

\section{Analysis of the yeast Sil1p and human HSPBP1 structures}

Multiple sequence alignment of human SIL1, S. cerevisiae Sil1p, and human HSPBP1 (UniProt ID Q9NZL4) using Clustal Omega was computed with the DNASTAR ${ }^{\circledR}$ MegAlign Pro software. This alignment has also been previously reported in (63). We computed pairwise structural alignments with the jFATCAT-rigid method (http://www.rcsb.org/pdb/workbench/workbench.do), using 3QML_chain C for Sc_Sillp (sequence length=262 aa), 1XQS_chain A for Hu_HSPBP1 (sequence length=264 aa), 3QML_chain A for Sc_Kar2p (sequence length=377 aa), and 1 XQS_chain D for $\mathrm{Hu}$ _HSP70 (sequence length=165 aa).

\section{Generation of MSS-associated SIL1 mutants using site-directed mutagenesis}

pSVL-SIL1 ${ }^{\text {WT }}$ was used as the template to generate the nine MSS-associated SIL1 mutations (Table 4-1), and site-directed mutagenesis was performed as previously reported (88) with a few adaptations. Briefly, Pwo DNA polymerase (identifier: 11644955001; Millipore Sigma, Burlington, MA) was used in the mutant strand synthesis PCR reaction, instead of the reported PfuTurbo DNA polymerase in the company protocol. Thermal cycling was performed using the DNA Engine ${ }^{\circledR}$ PTC-200 Gradient Thermal Cycler (Bio-Rad Laboratories, Inc., Hercules, CA) with the following parameters: $95^{\circ} \mathrm{C}$ for $30 \mathrm{~s}, 95^{\circ} \mathrm{C}$ for $30 \mathrm{~s}, 55^{\circ} \mathrm{C}$ for $60 \mathrm{~s}, 72^{\circ} \mathrm{C}$ for 5 minutes, repeated from step 2 for an additional 35 cycles, $72^{\circ} \mathrm{C}$ for 7 minutes, and held at $4{ }^{\circ} \mathrm{C}$ overnight. Following generation of the mutant strands, digestion of the amplified products was performed using $10 \mathrm{U} / \mu 1$ of DpnI (identifier: R0176S; New England Biolabs, Ipswich, MA) and incubated for 2 hours at $37^{\circ} \mathrm{C}$, following which bacterial cells were transformed. Sanger sequencing was performed to verify the generation of the respective mutant cDNA for expression in COS-1 cells. The forward primer sequences were used for site-directed mutagenesis are listed in Table 4-3, whereas the respective reverse complement of these sequences were used as the reverse primer. The p.L457P (c.1370T $>$ C) SIL1 mutant has been previously reported (88). Primers were obtained from ThermoFisher Scientific (Waltham, MA). 
Table 4-3. Primer sequences for the generation of SIL1 mutants using sitedirected mutagenesis.

\begin{tabular}{|c|c|}
\hline Nucleotide change & Forward primer sequence \\
\hline c. $274 \mathrm{C}>\mathrm{T}$ & 5'-ggctgtccctgcaggatcccacgtatggctgaatcttcagactgggg-3' \\
\hline c.617_618TC $>$ AA & 5'-gtttggaagagaagattgctgcgcaatttgatcttgaatattatgtccatc-3' \\
\hline c.691_696del & 5'-gctttcctttggtggtcttcaagtgaatgggetgaacagcacagagcccc-3' \\
\hline c. $892 \mathrm{C}>\mathrm{T}$ & $5^{\prime}$-cctgtttgcactgtgctccetgctgtgccacttcccetatgcceagcggc-3' \\
\hline c. $935 \mathrm{G}>\mathrm{A}$ & 5' -ccagcggcagttcctgaagctcggggagetgcaggtcctgaggaccetgg-3' \\
\hline c. $934 \mathrm{G}>\mathrm{A}$ & 5' -ccagcggcagttcctgaagctcgggaggctgcaggtcctgaggaccetgg-3' \\
\hline c. $1230 \_1244 \mathrm{del}$ & 5'-gcgtcctcetgaccacctgcegggaccccagctcggcaggacactggcc-3' \\
\hline c. $1367 \mathrm{~T}>\mathrm{A}$ & 5'-gctgctgggctctgtcaacagctagctgaaggagctgagatgaggcccc-3' \\
\hline
\end{tabular}

NOTE: The reverse complement of the respective forward primer sequence was used as the reverse primer. The cDNA substitution is indicated as bold and underlined in the forward primer sequence. 


\section{Cell culture, transfections, western blotting, and densitometric quantitations}

COS- 1 cells were maintained and transfected using GeneCellin (Bulldog Bio, Inc., Portsmouth, NH) as described previously (44). 450,000 COS-1 cells were seeded per p60 24 hours prior to transfection. Cells were transfected with $3 \mu \mathrm{g}$ per p60 of either wild-type or mutant SIL1 cDNA using GeneCellin, following the manufacturer's protocol. 24 hours after transfection, cells were harvested and lysed in $50 \mathrm{mM}$ Tris $(\mathrm{pH}$ 7.5) buffer containing $150 \mathrm{mM} \mathrm{NaCl}, 0.5 \%$ Nonidet P-40 and $0.5 \%$ deoxycholic acid, designated as the NP40 lysis buffer. The clarified lysates obtained after centrifugation at 21,130 $g$ for 20 minutes were designated as the NP40-soluble fraction. The NP40insoluble pellets were washed once with NP40 lysis buffer and then solubilized in $50 \mathrm{mM}$ Tris ( $\mathrm{pH} 8)$ buffer, containing 0.6\% SDS, sonicated for 4 minutes $(30 \mathrm{~s}$ ON-30 s OFF cycles) and incubated at $95^{\circ} \mathrm{C}$ for 10 minutes. All buffers contained $0.25 \mathrm{mM}$ phenylmethylsulphonyl fluoride (PMSF) and 1x complete protease inhibitor tablets without EDTA (Roche Applied Science, Indianapolis, IN, USA), as reported (194). 2\% $(\mathrm{v} / \mathrm{v})$ of the total NP40-soluble lysates and $10 \%(\mathrm{v} / \mathrm{v})$ of the total insoluble lysates were electrophoresed under reducing conditions on $8 \%$ gels. The following antibodies were used for immunoblotting: rabbit anti-SIL1 antisera (dilution of 1:1000; reported in (63, 64) and anti-GAPDH (dilution of 1:2500; clone 6C5; identifier: MAB374; EMD Millipore). GAPDH serves as the loading control. Western blots probed for SIL1 from three experimental trials were quantified using ImageQuant ${ }^{\mathrm{TM}}$ TL (GE Healthcare Life Sciences, Inc., Issaquah, WA). The values obtained from the NP40-soluble fractions were normalized to their respective GAPDH control values and graphically expressed as fold changes for the SIL1 mutants, relative to the wild-type SIL1 levels set to 1. The values obtained from the NP40-insoluble fractions were normalized to their respective NP40-soluble SIL1 levels that are corrected for equivalent loading, and graphically expressed as fold changes for the SIL1 mutants, relative to the wild-type SIL1 levels set to 1 .

For the analysis of SIL1 accumulation as higher molecular weight species under non-reducing and reducing conditions, COS-1 were transfected with either $3 \mu \mathrm{g}$ of empty pSVL, pSVL-SIL1 ${ }^{\mathrm{WT}}$, or pMT-BiP 44k alone (reported in (42)), or co-transfected with 2 $\mu \mathrm{g}$ of either wild-type or mutant SIL1 and $1 \mu \mathrm{g}$ of pMT-BiP 44k. 24 hours after transfection, cells were DSP-crosslinked, lysed in radioimmunoprecipitation assay (RIPA) buffer supplemented with 0.25 mM PMSF and 1x Roche complete protease inhibitor tablets without EDTA (Roche Applied Science, Indianapolis, IN, USA), as reported (59), electrophoresed under non-reducing and reducing conditions on an $8 \%$ gel, and probed for SIL1.

For the CHX-chase experiments, COS- 1 were transfected with $2 \mu \mathrm{g}$ per p60 of either wild-type or mutant SIL1 cDNA using GeneCellin, following the manufacturer's protocol. 24 hours after transfection, cells were treated with $5 \mu \mathrm{g} / \mathrm{ml} \mathrm{CHX} \mathrm{(identifier:}$ C4859; Millipore Sigma, Burlington, MA) to inhibit protein synthesis, and chased for zero-, three-, and six-hours, respectively. Cells were then lysed with NP40 lysis buffer and the NP40-soluble fractions were subjected to reducing electrophoresis and immunoblotted for SIL1 and HSC70 (1:1000 dilution; identifier: sc-1059, Santa Cruz 
Biotechnology). HSC70 serves as the loading control. These western blots were subjected to densitometric quantitation using ImageQuant ${ }^{\mathrm{TM}} \mathrm{TL}$. The values obtained for wild-type and mutant SIL1 levels at the three timepoints were normalized to their respective HSC70 values, and graphically expressed as percentage of SIL1 remaining at the three- and six-hour timepoints, relative to the levels of SIL1 at the zero timepoint set to $100 \%$. to transfections, and were either untransfected or transfected with $3 \mu \mathrm{g}$ per p60 of the p.R411_D415 del SIL1 mutant cDNA using GeneCellin, following the manufacturer's protocol. 24 hours after transfection, cells were harvested and lysed in RIPA buffer supplemented with $0.25 \mathrm{mM}$ PMSF and 1x Roche complete protease inhibitor tablets without EDTA (Roche Applied Science, Indianapolis, IN, USA), as reported previously, and electrophoresed under reducing conditions on an $8 \%$ gel, transferred onto a PVDF membrane, and probed for SIL1. 


\section{CHAPTER 5. DISCUSSION}

\section{Molecular Mechanisms Underlying Marinesco-Sjögren Syndrome}

\section{SIL1 mutations and the genetic heterogeneity associated with MSS}

Lagier-Tourenne et al. first mapped the MSS locus to chromosomal segment 5q31 (85). Senderek et al. and Anttonen et al. independently confirmed the genetic association of the disease phenotype with this locus, and further refined the $5 \mathrm{q} 31$ locus to identify mutations in the SIL1 gene in MSS patients of numerous ethnicities. In doing so they also excluded mutations in the 5q31-co-resident gene, SAR1B, which was previously also thought to be associated with MSS, and in the CCFDN (Congenital cataracts, facial dysmorphism, and neuropathy syndrome)-causing CTDPl gene, which serves as the major differential diagnosis of MSS $(64,86)$. Although a wide gamut of SIL1 mutations have been associated with MSS, patients present with a relatively homogeneous phenotype and almost invariably demonstrate the characteristic clinical symptoms of cerebellar ataxia, bilateral cataracts, intellectual disability, and myopathy (78). Thus, conforming with the hypothesis that these mutations eventually lead to a loss of functional SIL1, which is the root cause of MSS. Multiple subsequent studies have also observed an absence of any SIL1 mutations in patients with typical MSS, displaying the characteristic features of this disease, and individuals presenting atypical MSS with an unusual involvement of white matter and peripheral neuropathy amongst other features. This suggests that genetic heterogeneity in MSS with mutations in SIL1 plays a role in about $60 \%$ of the total cases $(64,86,115,195)$. Since typical MSS patients with or without SIL1 mutations display nearly identical clinical features, it is plausible that the genetic locus underlying the disease phenotype in the remaining $40 \%$ of MSS patients may function in the same pathway as SIL1. In support of this, loss of the BiP cochaperone, DNAJC3/ERdj6, in humans and mice leads to a similar neurodegeneration phenotype as MSS, including early-onset ataxia, intellectual disability, short stature, and pyramidal tract signs, in addition to diabetes mellitus due to apoptosis of pancreatic islet cells $(196,197)$. ERdj6 has also been reported to function as a modifier of MSS, and loss of this BiP co-chaperone alleviates the neurodegeneration phenotype of SIL1-deficient mice, whereas the partial loss of GRP170, the second ER-resident nucleotide exchange factor of BiP, seemed to exacerbate the same phenotype (91). In an extended analysis of 18 MSS-patient samples, Anttonen et al. ruled out mutations in HYOU1/GRP170, $H S P A 5 / B i P$, and in the gene encoding alanyl tRNA synthetase (AARS), which causes an MSS-like phenotype in mice (88). These results are in keeping with the embryonic lethality observed in $B i P$-null and GRP170-deficient mice, arguing that functionally inactivating mutations in either BiP or GRP170 are less likely to be observed in MSS patients since these individuals display an apparently normal lifespan $(35,198)$. In addition, mutations in the gene encoding the BiP-interacting protein, inositol polyphosphate-5-phosphatase K (INPP5K), reportedly leads to a congenital muscular

dystrophy with early-onset cataracts and mild intellectual disability that overlaps with the 
spectrum of MSS-associated features, suggesting that the INPP5K locus may have a role in atypical MSS (199).

\section{The neurological features of MSS}

The first mechanistic evidence demonstrating how the loss of SIL1 may cause the multisystem defects observed in MSS came from SIL1-deficient mice. Recapitulating the disease phenotype, mice with a homozygous disruption of Sill developed ataxia and displayed Purkinje cell loss, which was characterized by the accumulation of membranous autophagosomes, ubiquitinated protein aggregates, activation of the UPR, and apoptosis (87). Interestingly, SIL1 is widely expressed in many regions of the brain with the highest expression in the inferior olive and choroid plexus, yet the loss of SIL1 particularly affected the cerebellar Purkinje cells in lobules I-VIII and spared the lobule $\mathrm{X}$ and caudal lobule IX of the vestibulocerebellum, which represents a developmentally and functionally distinct region of the cerebellum (87). While BiP-deficiency is embryonic lethal, the non-lethal phenotype of SIL1-deficient mice was attributed to the redundancy of SIL1 with GRP170, which also demonstrates nucleotide exchange activity towards $\mathrm{BiP}(44,50,53,200)$. Consistent with this explanation, the reduction of GRP170 gene dosage worsens the cerebellar phenotype in SIL1-null mice and impacts the previously unaffected lobules IX and $\mathrm{X}$, whereas its transgenic overexpression is able to completely rescue the Purkinje cell degeneration, proving it to be a modifier of this disease along with ERdj6 (91). Although the transgenic overexpression of GRP170 seemed to completely ameliorate the cerebellar pathology, the endogenous ability of different tissue systems to dramatically upregulate GRP170 might be capped since it has been associated with cerebellar dysfunction and vacuolar degeneration in skeletal muscles $(200,201)$. Mild to moderate intellectual disability is a cardinal feature of MSS that precipitates relatively early in the course of this disease (78). Inaguma et al. demonstrated data suggesting that SIL1 is regulated in a spatiotemporal manner during brain development, and SIL1-depletion in ventricular zone progenitor cells of embryonic murine brains caused an abnormal cellular morphology, aberrant neuronal migration pattern with delayed kinetics, and slower axonal growth, resembling the phenotype observed upon BiP-knockdown in these cells and mutant-BiP knock-in mice (78, 90, 202). The migration defects observed upon SIL1-depletion were easily rescued by supplementation with human SIL1, whereas the MSS-associated SIL1 mutants, p.R411_D415del and p.L457P failed to do so, highlighting the pathophysiological significance of these SIL1 mutant proteins in MSS. The perturbation of the cortical neuronal cytoarchitecture, combined with the potential effects of SIL1 loss on neuronal protein quality control mediated by $\mathrm{BiP}$, may cause the varying degrees of intellectual impairment observed in MSS.

\section{The myopathy in MSS}

Multiple studies have characterized the myopathic changes observed in MSSpatient biopsies by electron microscopy and confirmed muscular dystrophy, including the 
variation in myofiber size, the presence of atrophic and hypertrophic fibers, autophagic membranous whorls, internalization of nuclei, depletion of the predominantly glycolytic, type IIB myofibers, and suggested that disturbed lysosomal function underlies the muscle pathology in MSS $(80-82,203,204)$. Using the two different mouse models of MSS, which disrupt nearly identical regions of Sill but yield dramatically different SIL1 proteins, we and Roos et al. have demonstrated that SIL1-disruption in mice strikingly phenocopies the ultrastructural features of the myopathy observed in MSS patients. Both these studies further established that loss of SIL1 leads to activation of the unfolded protein response that drives the upregulation of numerous ER chaperones, co-chaperones, and components of the ERAD machinery display, however, led to an autophagy impairment $(100,194)$. Autophagy plays a key role in regulating skeletal muscle mass, and the absence of an unfettered response leads to loss of muscle mass and force, protein aggregation, presence of abnormal membranous structures, and dilated sarcoplasmic reticulum (205), which are all in keeping with the observations in SIL1-disrupted mice. We additionally provide physiological evidence for the loss of muscle strength with aging in Sill $^{\mathrm{Gt}}$ mice and use a proteomics approach to identify proteins that are deregulated in the SIL1-deficient skeletal muscles. This led us to the discovery of the ER-trafficking proteins, insulin and IGF-1 receptors, whose biosynthesis and maturation were compromised in the absence of SIL1, and a physiological upregulation of PI3K-AKTmTOR signaling at steady state in ad libitum fed mice. In numerous conditions, such as cancer, Cushing's syndrome, denervation, diabetes, disuse atrophy, fasting, sepsis, and uremia, skeletal muscles atrophy through activation of the ubiquitin-proteasomal pathway via the FOXO transcription factors. These transcription factors upregulate the expression of two E3 ubiquitin-ligases, Atrogin-1 and MuRF1, that serve as master regulators of the ubiquitin-proteasomal process (206). An impending question that our study raises is why Atrogin-1 and MuRF1 transcripts are not noticeably upregulated in Sill $^{\text {Gt }}$ skeletal muscles at the 15-month timepoint, which corresponds to a state of ongoing myopathy? There are two potential explanations for our observations. (1) Since we used whole skeletal muscle as our source of mRNA for Atrogin-1 and MuRF1 transcript analysis, their expression represents a gross average, which includes atrophic, hypertrophic, and apparently normal myofibers. It is likely that the number of myofibers undergoing marked degeneration and atrophy at a given time relative to the entire myofiber population, which are the source of increased levels of Atrogin-1 and MuRF1 transcripts, are not very high. For the remaining hypertrophic and apparently normal myofibers, as our western blotting data demonstrates, we observe a gross upregulation of PI3K-AKT-mTOR signaling, which inhibits FOXO's localization to the nucleus and thereby its transcriptional activity, suggesting that Atrogin-1 and MuRF1 transcript levels may be spatially distinct between myofibers. The observation of hypertrophic myofibers conforms with the physiological activation of PI3K-AKT-mTOR axis, due to its well established role in mediating skeletal muscle hypertrophy (158). It is noteworthy that the myopathy observed in Sill ${ }^{\mathrm{Gt}}$ mice mimics numerous features of sarcopenia, the agingrelated progressive loss of skeletal muscle mass and function (reviewed in (137), and AKT-mediated downregulation of Atrogin-1 and MuRF1 is one such feature of this process (207). (2) Our skeletal muscle data from ad libitum fed Sill ${ }^{\mathrm{Gt}}$ mice is consistent with the data from ad libitum fed, muscle-specific IR and IGF1R knockout mice, which displayed a greater upregulation of Atrogin-1 and $M u R F 1$ relative to control mice in the 
fasted state, but displayed no significant difference upon being randomly fed (208). The authors further elaborate that the upregulation of Atrogin-1 and MuRF1 transcripts are an acute response to skeletal muscle atrophy, which is not observed under chronic myopathic conditions (208), offering an additional explanation of why the chronic myopathy observed upon loss of SIL1 does not trigger a measurably sustained upregulation of Atrogin-1 and MuRF1 transcripts.

While the constitutive activation of mTORC1 signaling alone has been shown to trigger skeletal muscle atrophy and may explain the preferential loss of glycolytic, type IIb fibers (209), similar to what is observed in MSS patients and in the MSS-mouse model $(194,203)$, it is puzzling to understand how increased PI3K-AKT signaling may be achieved in the face of decreased levels of its upstream transducers, IR and IGF1R. It is possible that if other receptor tyrosine kinases, such as EGFR, remain unaffected by the disruption of ER proteostasis in SIL1-deficient skeletal muscles, or the depletion of the negative regulators of this pathway, such as the phosphoinositide phosphatases, may cause a sustained activation of PI3K-AKT-mTOR signaling. The former has also been suggested for MIGIRKO mice, which display an increase in the levels of skeletal muscle EGFR expression (156). In keeping with these hypotheses, our proteomics data indicated a $21 \%$ increase in EGFR levels (fold change $=1.21, P=0.004, \log _{2}$ ratio $=0.28$ ), and a $39 \%$ decrease in the levels of inositol polyphosphate 4-phosphatase type II (INPP4B; fold change $=0.61, P=1.42 \mathrm{E}-06, \log _{2}$ ratio $\left.=-0.70\right)$. INPP4B is highly expressed in skeletal muscles and regulates the hydrolysis of phosphatidylinositol 3,4-bisphosphate to phosphatidylinositol 3-phosphate, which serve as essential signaling activators of PI3KAKT signaling together with phosphatidylinositol 3,4-bisphosphate (210). Thus, the loss of INPP4B, which has also been linked to PI3K-AKT hyperactivation (211), together with increased EGFR levels, serve as promising mechanisms that may cause sustained PI3K-AKT signaling in spite of a decrease in IR and IGF1R levels.

\section{The metabolic features of MSS}

Metabolic features have been rarely reported for MSS patients (85), but it remains unclear if either typical or atypical MSS indeed presents without any metabolic complications or if these features have not been thoroughly characterized. Both SIL1 and $\mathrm{BiP}$ are highly expressed in secretory tissues, including the pancreas $(63,105)$. More recently, using the Sill ${ }^{\mathrm{wz}}$ mouse model, it has been determined that SIL1 is required to maintain islet insulin content, islet size, glucose tolerance, and ER homeostasis in pancreatic $\beta$ cells (105). Modulation of the levels of SIL1 correlated well with insulin content and the ability of pancreatic beta cells to secrete insulin. Importantly, loss of SIL1rendered the Sill ${ }^{\mathrm{wz}}$ mice more vulnerable to streptozotocin-induced type I diabetes, and led to glucose intolerance upon high-fat diet feeding (105). However, it remains unclear if the irregularities in insulin secretion and synthesis in Sil $\mathrm{wz}^{\mathrm{wz}}$ mice are also accompanied by aberrant glucose sensing. In support of the latter possibility, we observed abnormal levels of numerous plasma membrane proteins and metabolic enzymes, both of which play an essential role in the generating the signal for insulin secretion (212). Largely, these results are in keeping with our observation of diffused 
perinuclear vacuolization in the pancreas of aged Sill $^{\mathrm{Gt}}$ mice (213), and our evidence for glucose intolerance and insulin resistance in these mice (Appendix Figure A-1). One study has reported apparently normal cerebral glucose metabolic rates using radiolabeled glucose and PET-CT (214). The brains of Sill ${ }^{\mathrm{Gt}}$ mice displayed defects in radiolabeled glucose uptake as well only upon stimulation with insulin, which suggests an abnormal insulin-responsive glucose uptake mechanism (Appendix Figure A-2). If the two mouse models of MSS do indeed recapitulate the disease in its entirety, these results emphasize the requirement of a more thorough clinical analysis of the metabolic phenotype in MSS patients, since pancreatic defects in insulin synthesis may be easily circumvented by recombinant insulin supplementations.

\section{Differences Between the Two Murine Models of MSS}

It is worth noting that our data obtained in $\mathrm{Sill}^{\mathrm{Gt}}$ mice differ in several significant aspects from two previous reports that utilize Sill ${ }^{\mathrm{wz}}$ mice. Both the Sill ${ }^{\mathrm{wz}}$ (100) and $\mathrm{Sill}^{\mathrm{Gt}}$ (this study) mice develop a myopathy, which is characterized by the accumulation of unfolded proteins, increases in some ER chaperones and components of cellular degradation systems, and very similar changes in the cyto-architecture of quadriceps, as detected by EM. Although there were changes in the onset and magnitude of UPR activation between the two models, more significantly, the myopathy in the Sill ${ }^{\mathrm{wz}}$ mouse was diagnosed to be of neurogenic origin, whereas we found no significant evidence of a neurogenic contribution to the Sil1 ${ }^{\mathrm{Gt}}$ myopathy. We do not know the reason for this difference, but it is conceivable that the effect on individual proteins varies between these models. For instance, if a neurotransmitter or receptor was affected in Sill ${ }^{\mathrm{wz}}$ mice but not $S i l 1^{\mathrm{Gt}}$ mice it could lead to a distinct etiology. Along these lines, another study using the Sill ${ }^{\mathrm{wz}}$ mouse found that glucose-stimulated secretion of insulin from pancreatic cells was impaired (105). Insulin is a known BiP client (215), and thus it is conceivable that the loss of SIL1 interferes with BiP release and prevents its secretion; although this was not directly measured. Nonetheless, this hypothesis is supported by SIL1 knock-down and over-expression studies in a pancreatic cell line, where basal levels of proinsulin and secretion of insulin corresponded to Sill levels (105). In contrast, we found both basal and glucose-stimulated blood insulin levels were very similar in age-matched wild-type

and Sill ${ }^{\mathrm{Gt}}$ mice. This argues that the Sill ${ }^{\mathrm{Gt}}$ mice did not have a significant problem with folding or secreting insulin and provides an example of a specific BiP client protein that is affected distinctly by SIL1 loss. IR and IGF1R represent two other proteins that might be affected disparately in the two mouse models. Although neither protein was directly examined in the quadriceps of Sill ${ }^{\mathrm{wz}}$ mice (114), they had a normal insulin tolerance test, arguing that, unlike the Sill ${ }^{\mathrm{Gt}}$ mice, insulin-mediated glucose uptake was not affected. There are two unique aspects of these mice that could contribute to these inconsistencies. First, the methods used for disrupting the Sill gene in these two mouse models result in two distinct SIL1 fusion proteins. However, both SIL1 mutant proteins are unstable and are unlikely to underlie the differences we have detected. In support of this, over 46 SIL1 mutations have been identified to date in individuals with MSS, which result in very distinct mutant proteins, but there is no correlative evidence that disease symptoms vary according to SIL1 mutations $(99,115)$. The second difference is that the Sill ${ }^{\mathrm{wz}}$ mouse 
arose from a spontaneous transposon insertion into the Sill gene in a CXB5/ByJ mouse, whereas gene-trap methodology was used to create the Sill ${ }^{\mathrm{Gt}}$ mouse on the C57/BL6 background. Thus, it is plausible that the unique genetic backgrounds of the two mice may play a bigger role in contributing to the variations in data.

\section{Why Does the Loss of SIL1 in Humans and Mice Selectively Affect Some Tissues, While Sparing Others?}

\section{The SIL1 expression-effect paradox}

The selective vulnerability of certain tissues, such as the cerebellum, cerebral cortex, skeletal muscles, pancreas, and lens, to the loss of SIL1 remains a puzzling question for this disease $(64,78,86,87,100,105,194)$. In fact, it appears that highly secretory tissues, such as the kidney, liver, placenta, and plasma cells, where one might expect adverse consequences when a component of the chaperone machinery is lost, are relatively less affected by SIL1 loss (216). Instead the effects are greatest in the Purkinje and glial cells of the cerebellum, the lens epithelial cells, and skeletal muscles (78), which are not generally associated with the synthesis of industrial amounts of secretory proteins that are BiP substrates. It is possible that the more classical secretory tissues have a greater number of failsafe mechanisms built into their cellular systems and are better able to respond to or accommodate changes in the client load of the ER.

\section{Possibilities for selective vulnerability of certain tissues in MSS}

In line with this reasoning, it is also possible that within a given cell type, cellular subtypes may express different proteostasis programs. This can be exemplified by the differences between motor neurons innervating fast or slow skeletal muscles (135). The ER of fast motor neurons express relatively higher levels of components of the ERAD machinery, such as VIMP, EDEM1, SEL1L, and OS9, and ER stress sensors, such as PERK, IRE1, EIF2 $\alpha$. On the contrary, the ER of slow motor neurons express higher levels of chaperones and co-chaperones, such as SIL1, ERP29, CRT, BiP, Grp94, and Grp170 (135). These fast motor neurons display low-excitability, but are highly phasic and generate short-lived powerful muscle contractions, whereas the slow motor neurons are smaller in size, highly excitable, and extremely resistant to fatigue. In keeping with their functional demands, the differences in their expression patterns of proteostasis components would elicit dramatically distinct responses upon disruption of ER proteostasis or the introduction of misfolded proteins for example. This would result in differential vulnerabilities of these motor neuron subtypes, as demonstrated by the selective vulnerability of fast motor neurons in fALS. Thus, it is possible that the metabolically distinct glycolytic and oxidative skeletal muscle subtypes, the evolutionarily distinct lobule $\mathrm{X}$ and ventral lobule IX of the vestibulocerebellum compared to the other cerebellar lobules, and tissues that are affected by the loss of SIL1 versus those that remain largely unaffected, all display evolutionarily distinct proteostasis 
programs based on their function, which may also govern why certain tissues are particularly vulnerable in MSS.

\section{An unexpected new role for SIL1 as a BiP-reductase}

Alternatively, an intriguing possibility comes from the ability of different tissue systems to handle oxidative stress distinctly. Recent in vitro studies in yeast have shown that Kar2p, the yeast homolog of SIL1, is a direct sensor of changes in the redox balance within the ER via a conserved cysteine thiol within Kar2p's NBD (cysteine-63) that is reversibly oxidized by reactive oxygen species $(67,217)$. The modification of this cysteine residue inhibits BiP's ATPase activity, but accentuated its ability to bind to misfolded proteins and prevent protein aggregation by decoupling its holdase activity from the regulation by BiP's nucleotide-bound state $(67,217)$. In a subsequent study, it was shown that the nucleotide exchange factor, Sil1p, can also act as a reductase for oxidized Kar2p, thereby restoring its chaperone activity during recovery from oxidative stress (66). Sillp's reductase activity is mediated through two N-terminal cysteines (cysteines 52 and 57). The yeast homolog of Grp170, Lhs1p, was unable to reduce modified Kar2p and stimulate its ATPase activity (66), thus, implying that this reductase activity is specific for Sil1p and not a common feature of Kar2p's NEFs.

It has further been demonstrated that Sillp's reductase activity is in turn regulated by its N-glycosylation status. Both Sillp and Lhs $1 p$ are glycoproteins, and the Nglycosylation of Sillp, but not of Lhs1p, is diminished during reductive ER stress (218). The N-glycosylation of Sillp is primarily mediated by the yeast oligosaccharyltransferase, Ost3, whereas Lhs1p glycosylation is largely Ost3independent. During redox imbalance and stress, the unglycosylated Sillp not only retained functionality but was apparently more effective at compensating for Lhs $1 \mathrm{p}$ loss than its glycosylated form, outlining a possibility during redox stress where Sillp is crucial to facilitate nucleotide exchange for Kar2p when Lhs $1 p$ becomes limiting (218). Adding to this line of thought, it has also been recently shown that Sillp not only facilitates nucleotide exchange for Kar2p, but also acts as its conformational regulator and plays a more active role in the release of substrates from BiP than previously anticipated (219).

Although there are two N-terminal cysteines that are highly conserved in mammalian SIL1 sequences, algorithms for signal sequence cleavage predict that they are not retained in the mature protein. If in fact they are part of the mature SIL1 protein, a similar reductase activity for mammalian SIL1 could readily explain the delayed effects on secretory pathway protein expression, as the need for this reductase activity might be dependent on accumulated oxidative insults to the ER, leading to the oxidization and inactivation of BiP. Further investigation is required to determine if a mammalian SIL1 possesses a reductase activity for $\mathrm{BiP}$ and whether this is a plausible explanation for the selective vulnerabilities of certain tissues to the loss of SIL1 in MSS. It is further tempting to speculate if mutations in proteins that may be involved in the redox 
homeostasis function of SIL1 could contribute to the genetic heterogeneity observed in MSS.

\section{Potential Strategies to Treat MSS}

No treatment regimens that are specifically targeted to mitigate the pathological mechanisms underlying MSS currently exist, and MSS is managed symptomatically with cataract extraction and hormone replacement therapy for primary gonadal failure (220). The logistic challenges for the treatment of MSS are synonymous with the treatment of any ultra-rare disease, but the widespread distribution of MSS-associated SIL1 mutations, genetic and clinical heterogeneity, and the lack of a thorough understanding of the pathomechanisms of all the primary defects associated with MSS particularly hinder the exploration of potential therapeutic strategies. Thus, the pharmacotherapeutic approach for MSS to be feasible must be applicable to the majority of patient population with varying genetic heterogeneity and clinical presentation of symptoms, as compared to a more directed mutation-specific approach, the latter of which has shown remarkable results in the correction of the cystic fibrosis-causing CFTR mutants (221). The two murine models and the zebrafish model of MSS faithfully recapitulate disease complexities and offer promise for expediting drug discovery studies $(87,100,194,222)$.

\section{Chemical chaperones}

To date, two studies have primarily undertaken different approaches to mitigate some of the pathologies associated with MSS. Kashimada et al. detected that SIL1deficient, MSS-patient derived, lymphoblastoid cell lines displayed a greater upregulation of BiP and spliced XBP1 when treated with a pharmacological ER stressor, and were more prone to ER stress-induced cell death (223). Although it has been reported that chemical stresses fail to mimic the unfolded protein response that originates from the accumulation of unfolded or misfolded proteins (224), pretreatment of patient-derived LBLs treated with the hydrophobic chemical chaperone, TUDCA, significantly reduced apoptotic cell death when subsequently exposed to the chemical stressor, tunicamycin, and displayed a significant reduction in mitochondrial depolarization and caspase activation (223). Contrarily, pretreatment with the branch chain fatty acid, valproate, the glucocorticoid, dexamethasone, and an alternative chemical chaperone, PBA, did not have a major effect on apoptotic cell death, in spite of numerous reports indicating their ability to mitigate the ER stress response $(225,226)$. This suggests that the mechanism by which these small molecules prevent cell death in MSS-derived LBLs depends on their mode of action rather than their ability to curb the ER stress response, although this point has not been thoroughly investigated for MSS-derived LBLs. Both PBA and TUDCA are hydrophobic chemical chaperones, whose proposed mechanisms of action involves their interaction with exposed hydrophobic protein regions, which are generally buried in correctly folded protein conformations, but surface exposed in misfolded or unfolded states. Both PBA and TUDCA are orally bioavailable, BBB-permeable, have a no-to-low toxicity profile, and despite having manifold similar features display a marked 
difference in their ability to prevent cell death in SIL1-deficient MSS-LBLs. This difference can be attributed, at least partially, to the differences in their mechanism-ofaction supplementary to their role as a chemical chaperone. TUDCA is a bile acid and has been shown to mediate its cytoprotective effects by reducing ROS formation, preventing mitochondrial dysfunction, and inhibiting apoptosis via the intrinsic and extrinsic pathway (180), which is consistent with what the authors observe for the TUDCA-treatment of patient-derived LBLs. In keeping with these results, our CRISPR/Cas9-engineered SIL1-null C2C12 murine myoblasts (Appendix Figures A-3 and A-4) will be a useful tool in evaluating the efficacy of both chemical chaperones in a cell type that is affected in MSS.

\section{Modulation of the PERK pathway}

The PERK-CHOP branch of the unfolded protein response has been demonstrated to be activate in the cerebellar Purkinje cells of $S i l 1^{\mathrm{wz}}$ mice prior to their degeneration $(87,227)$. Grande et al. tested the therapeutic efficacy of an orally bioavailable PERK inhibitor, GSK2606414, which would circumvent the translational attenuation response mediated by this UPR branch (228). Chronical treatment of Sill ${ }^{\mathrm{wz}}$ mice with GSK2606414, beginning from a presymptomatic stage, significantly delayed the onset of physiological and biochemical signs of cerebellar degeneration and partially modulated the myopathy in these mice but did not prevent it altogether, which is in keeping with a previous report demonstrating that $C H O P$ deletion was not able to rescue Purkinje cell loss and ataxia in $\operatorname{Sill}^{\mathrm{wz}}$ mice $(91,227)$. The authors note that although the treatment with GSK2606414 was effective at PERK inhibition, the cerebellar cells did not display robust signs of ATF6 and XBP1 activation, which increases ER's proteostatic capacity and may be crucial in mitigating ER stress after rescuing protein synthesis by PERK inhibition. Surprisingly, it was recently shown that GSK2606414 is also a potent inhibitor of Receptor-interacting serine/threonine-protein kinase 1 (RIPK1), whose role in mediating cell death via apoptosis and necroptosis is well established (229). Thus, it remains unclear if the treatment with GSK2606414 partially prolongs the asymptomatic phase of cerebellar pathology by mediating PERK inhibition or by inhibiting cell death via RIPK1. Nonetheless, modulation of the PERK branch may be a viable strategy for rescuing the pathology in the MSS-affected tissues from a conceptual standpoint and need to be carefully evaluated in the available models of this disease. To this end, the recently discovered Sephin1 and Raphin1 inhibitors of the phosphatases that negatively regulate eIF $2 \alpha$ phosphorylation levels, causing a transient translational halt and providing proteostatic respite to cells, have demonstrated promise in preventing multiple proteostasis diseases, such as Charcot-Marie-Tooth 1B syndrome, amyotrophic lateral sclerosis, and Huntington's disease $(230,231)$. 


\section{The Role of SIL1 in the Pathogenesis of Other Diseases}

\section{SIL1 and neurodegenerative diseases}

Since SIL1 plays a crucial role in maintaining ER homeostasis in neurons, skeletal muscles, pancreas, and other cell types that are affected in MSS, it did not come as a surprise when SIL1 was implicated as a modifier in ALS (135). SIL1 haploinsufficiency in SOD1 mutant (SOD1-G93A) mice substantially accentuated ER stress and the associated pathology, rendering a previously resistant motor neuron subtype now vulnerable to ER dysfunction and disease manifestation. On the other hand, SIL1 expression was reduced in a mutant TDP-43 (TDP-43 ${ }^{A 315 T}$ ) fALS murine model, and co-localized with mutant TDP-43 in stress granule-like structures. Conversely, AAV-mediated SIL1-overexpression rescued denervation defects, reduced cellular stress

and increased survival (135). In fact, total SIL1 levels were also found to be decreased in the cortex and hippocampus of a murine model of Alzheimer's disease (Tg2576) (232), whereas the surviving hippocampal neuronal population in Alzheimer's disease autopsies displayed higher SIL1 levels (233). Overall, these results suggest a cytoprotective role of SIL1 in ALS and Alzheimer's disease.

\section{SIL1 and gliomas, the most common central nervous system tumors}

Recently, Xu et al. revealed that SIL1 was expressed at higher levels in glioblastoma multiforme and brain lower grade gliomas, accounting for approximately $66 \%$ of patient samples, and was further upregulated in grade IV gliomas compared to grades I-III (234). Downregulation of SIL1 using siRNA in the glioblastoma cell line U251 caused a significant inhibition of cell proliferation, migration, AKT-mTOR signaling, and triggered apoptosis without altering their cell cycle dynamics. This study and our work on the loss of SIL1 in skeletal muscles emphasize the ability of SIL1 to modulate AKT-mTOR signaling, although with opposing outcomes, which may be explained by differences in the levels of regulators of this signaling cascade. Overall, these results shed light on the potential beneficiary role of SIL1 in a subset of gliomas and further offer insights into a plausible approach by which glioma progression could be curtailed (234). In line with these results, it is tempting to speculate if MSS patients are resistant to these central nervous system tumor subtypes. Nevertheless, the role of SIL1 in maintaining tissue homeostasis, especially for neuronal cells, is evidently clear.

\section{Conclusions}

In toto, our data demonstrates that a subset of MSS-associated SIL1 mutations, which alter less than six amino acids, may dramatically alter the protein microenvironment and disrupt intramolecular interactions, such that it alters the folding properties of SIL1 and renders it aggregation-prone. This offers a potential explanation of the mechanism(s) by which mutations in SIL 1 cause a loss of its function. We confirm 
that the C57BL6 Sill ${ }^{\mathrm{Gt}}$ mouse model, which harbors a genetic disruption of the exon 9 and exon 10 resident BiP-interaction sites, recapitulate numerous aspects of the MSSphenotype and represents a valid preclinical model system to investigate the MSSassociated pathology and explore pharmacotherapeutic strategies. We further elucidate the molecular mechanisms by which loss of SIL1 leads to skeletal muscle myopathy and muscular weakness with aging, identifying crucial regulators of muscle function that are deregulated in SIL1-deficient skeletal muscles. We further provide data demonstrating that contrary to popular belief, loss of SIL1 does not necessarily affect all BiP's clientele, but may rather depend on additional factors, such as aging and redox imbalance. 


\section{LIST OF REFERENCES}

1. Braakman I, Bulleid NJ. Protein Folding and Modification in the Mammalian Endoplasmic Reticulum. Annual review of biochemistry. 2011;80(1):71-99. doi: 10.1146/annurev-biochem-062209-093836. PubMed PMID: 21495850.

2. Janda CY, Li J, Oubridge C, Hernández H, Robinson CV, Nagai K. Recognition of a signal peptide by the signal recognition particle. Nature. 2010;465:507. doi: $10.1038 /$ nature 08870 https://www.nature.com/articles/nature08870\#supplementary-information.

3. Chio US, Cho H, Shan S-o. Mechanisms of Tail-Anchored Membrane Protein Targeting and Insertion. Annual review of cell and developmental biology. 2017;33(1):417-38. doi: 10.1146/annurev-cellbio-100616-060839. PubMed PMID: 28992441.

4. Lindquist SL, Kelly JW. Chemical and Biological Approaches for Adapting Proteostasis to Ameliorate Protein Misfolding and Aggregation DiseasesProgress and Prognosis. Cold Spring Harbor perspectives in biology. 2011;3(12):a004507. doi: 10.1101/cshperspect.a004507. PubMed PMID: PMC3225948.

5. Klaips CL, Jayaraj GG, Hartl FU. Pathways of cellular proteostasis in aging and disease. J Cell Biol. 2017. Epub 2017/11/12. doi: 10.1083/jcb.201709072. PubMed PMID: 29127110.

6. Bartlett AI, Radford SE. An expanding arsenal of experimental methods yields an explosion of insights into protein folding mechanisms. Nature structural \& molecular biology. 2009;16(6):582-8. Epub 2009/06/06. doi: 10.1038/nsmb.1592. PubMed PMID: 19491935.

7. Woolhead CA, McCormick PJ, Johnson AE. Nascent membrane and secretory proteins differ in FRET-detected folding far inside the ribosome and in their exposure to ribosomal proteins. Cell. 2004;116(5):725-36. Epub 2004/03/10. PubMed PMID: 15006354.

8. Hwang C, Sinskey AJ, Lodish HF. Oxidized redox state of glutathione in the endoplasmic reticulum. Science. 1992;257(5076):1496-502. Epub 1992/09/11. PubMed PMID: 1523409.

9. Braakman I, Hebert DN. Protein folding in the endoplasmic reticulum. Cold Spring Harbor perspectives in biology. 2013;5(5):a013201. Epub 2013/05/03. doi: 10.1101/cshperspect.a013201. PubMed PMID: 23637286; PubMed Central PMCID: PMCPMC3632058. 
10. Ruggiano A, Foresti O, Carvalho P. ER-associated degradation: Protein quality control and beyond. The Journal of Cell Biology. 2014;204(6):869-79. doi: 10.1083/jcb.201312042.

11. Nakatsukasa K, Kamura T, Brodsky JL. Recent technical developments in the study of ER-associated degradation. Current opinion in cell biology. 2014;29(Supplement C):82-91. doi: https://doi.org/10.1016/j.ceb.2014.04.008.

12. Kruse KB, Brodsky JL, McCracken AA. Characterization of an ERAD Gene as VPS30/ATG6 Reveals Two Alternative and Functionally Distinct Protein Quality Control Pathways: One for Soluble Z Variant of Human $\alpha-1$ Proteinase Inhibitor (A1PiZ) and Another for Aggregates of A1PiZ. Molecular biology of the cell. 2006;17(1):203-12. doi: 10.1091/mbc.E04-09-0779.

13. Ishida Y, Yamamoto A, Kitamura A, Lamandé SR, Yoshimori T, Bateman JF, et al. Autophagic Elimination of Misfolded Procollagen Aggregates in the Endoplasmic Reticulum as a Means of Cell Protection. Molecular biology of the cell. 2009;20(11):2744-54. doi: 10.1091/mbc.E08-11-1092.

14. Schuck S, Gallagher CM, Walter P. ER-phagy mediates selective degradation of endoplasmic reticulum independently of the core autophagy machinery. Journal of cell science. $2014 ; 127(18): 4078-88$. doi: 10.1242/jcs. 154716 .

15. Tannous A, Pisoni GB, Hebert DN, Molinari M. N-linked sugar-regulated protein folding and quality control in the ER. Seminars in Cell \& Developmental Biology. 2015;41(Supplement C):79-89. doi: https://doi.org/10.1016/j.semcdb.2014.12.001.

16. Wijeyesakere SJ, Rizvi SM, Raghavan M. Glycan-dependent and -independent interactions contribute to cellular substrate recruitment by calreticulin. The Journal of biological chemistry. 2013;288(49):35104-16. Epub 2013/10/09. doi: 10.1074/jbc.M113.507921. PubMed PMID: 24100026; PubMed Central PMCID: PMCPMC3853262.

17. Saito Y, Ihara Y, Leach MR, Cohen-Doyle MF, Williams DB. Calreticulin functions $<$ em $>$ in vitro $</$ em $>$ as a molecular chaperone for both glycosylated and non-glycosylated proteins. The EMBO journal. 1999;18(23):6718-29. doi: 10.1093/emboj/18.23.6718.

18. Danilczyk UG, Williams DB. The lectin chaperone calnexin utilizes polypeptidebased interactions to associate with many of its substrates in vivo. The Journal of biological chemistry. 2001;276(27):25532-40. Epub 2001/05/05. doi: 10.1074/jbc.M100270200. PubMed PMID: 11337494.

19. Aebi M, Bernasconi R, Clerc S, Molinari M. N-glycan structures: recognition and processing in the ER. Trends in biochemical sciences. 2010;35(2):74-82. doi: https://doi.org/10.1016/j.tibs.2009.10.001. 
20. Haas IG, Wabl M. Immunoglobulin heavy chain binding protein. Nature. 1983;306(5941):387-9. Epub 1983/11/24. PubMed PMID: 6417546.

21. Bole DG, Hendershot LM, Kearney JF. Posttranslational association of immunoglobulin heavy chain binding protein with nascent heavy chains in nonsecreting and secreting hybridomas. J Cell Biol. 1986;102(5):1558-66. Epub 1986/05/01. PubMed PMID: 3084497; PubMed Central PMCID: PMCPMC2114236.

22. Hendershot LM, Kearney JF. A role for human heavy chain binding protein in the developmental regulation of immunoglobin transport. Molecular immunology. 1988;25(6):585-95. Epub 1988/06/01. PubMed PMID: 3139995.

23. Skowronek MH, Hendershot LM, Haas IG. The variable domain of nonassembled Ig light chains determines both their half-life and binding to the chaperone BiP. Proceedings of the National Academy of Sciences of the United States of America. 1998;95(4):1574-8. Epub 1998/03/21. PubMed PMID: 9465057; PubMed Central PMCID: PMCPMC19100.

24. Hellman R, Vanhove M, Lejeune A, Stevens FJ, Hendershot LM. The in vivo association of BiP with newly synthesized proteins is dependent on the rate and stability of folding and not simply on the presence of sequences that can bind to BiP. J Cell Biol. 1999;144(1):21-30. Epub 1999/01/13. PubMed PMID: 9885241; PubMed Central PMCID: PMCPMC2148116.

25. Hendershot LM, Ting J, Lee AS. Identity of the immunoglobulin heavy-chainbinding protein with the 78,000-dalton glucose-regulated protein and the role of posttranslational modifications in its binding function. Molecular and cellular biology. 1988;8(10):4250-6. Epub 1988/10/01. PubMed PMID: 3141786; PubMed Central PMCID: PMCPMC365497.

26. Morris JA, Dorner AJ, Edwards CA, Hendershot LM, Kaufman RJ. Immunoglobulin binding protein $(\mathrm{BiP})$ function is required to protect cells from endoplasmic reticulum stress but is not required for the secretion of selective proteins. The Journal of biological chemistry. 1997;272(7):4327-34. Epub 1997/02/14. PubMed PMID: 9020152.

27. Freiden PJ, Gaut JR, Hendershot LM. Interconversion of three differentially modified and assembled forms of BiP. The EMBO journal. 1992;11(1):63-70. Epub 1992/01/01. PubMed PMID: 1740116; PubMed Central PMCID: PMCPMC556426.

28. Preissler S, Rato C, Perera L, Saudek V, Ron D. FICD acts bifunctionally to AMPylate and de-AMPylate the endoplasmic reticulum chaperone BiP. Nature structural \& molecular biology. 2017;24(1):23-9. Epub 2016/12/06. doi: 10.1038/nsmb.3337. PubMed PMID: 27918543; PubMed Central PMCID: PMCPMC5221731. 
29. Broncel M, Serwa RA, Bunney TD, Katan M, Tate EW. Global Profiling of Huntingtin-associated protein E (HYPE)-Mediated AMPylation through a Chemical Proteomic Approach. Molecular \& cellular proteomics : MCP. 2016;15(2):715-25. Epub 2015/11/26. doi: 10.1074/mcp.O115.054429. PubMed PMID: 26604261; PubMed Central PMCID: PMCPMC4739684.

30. Sanyal A, Chen AJ, Nakayasu ES, Lazar CS, Zbornik EA, Worby CA, et al. A novel link between Fic (filamentation induced by cAMP)-mediated adenylylation/AMPylation and the unfolded protein response. The Journal of biological chemistry. 2015;290(13):8482-99. Epub 2015/01/21. doi: 10.1074/jbc.M1 14.618348. PubMed PMID: 25601083; PubMed Central PMCID: PMCPMC4375499.

31. Preissler S, Rato C, Chen R, Antrobus R, Ding S, Fearnley IM, et al. AMPylation matches BiP activity to client protein load in the endoplasmic reticulum. Elife. 2015;4:e12621. Epub 2015/12/18. doi: 10.7554/eLife.12621. PubMed PMID: 26673894; PubMed Central PMCID: PMCPMC4739761.

32. Ham H, Woolery AR, Tracy C, Stenesen D, Kramer H, Orth K. Unfolded protein response-regulated Drosophila Fic (dFic) protein reversibly AMPylates BiP chaperone during endoplasmic reticulum homeostasis. The Journal of biological chemistry. 2014;289(52):36059-69. Epub 2014/11/15. doi: 10.1074/jbc.M1 14.612515. PubMed PMID: 25395623; PubMed Central PMCID: PMCPMC4276871.

33. Behnke J, Feige MJ, Hendershot LM. BiP and its nucleotide exchange factors Grp170 and Sil1: mechanisms of action and biological functions. Journal of molecular biology. 2015;427(7):1589-608. Epub 2015/02/24. doi: 10.1016/j.jmb.2015.02.011. PubMed PMID: 25698114; PubMed Central PMCID: PMCPMC4356644.

34. Lee AS. The glucose-regulated proteins: stress induction and clinical applications. Trends in biochemical sciences. 2001;26(8):504-10. Epub 2001/08/16. PubMed PMID: 11504627.

35. Luo $\mathrm{S}$, Mao C, Lee B, Lee AS. GRP78/BiP is required for cell proliferation and protecting the inner cell mass from apoptosis during early mouse embryonic development. Molecular and cellular biology. 2006;26(15):5688-97. Epub 2006/07/19. doi: 10.1128/mcb.00779-06. PubMed PMID: 16847323; PubMed Central PMCID: PMCPMC1592753.

36. Paton AW, Beddoe T, Thorpe CM, Whisstock JC, Wilce MC, Rossjohn J, et al. AB5 subtilase cytotoxin inactivates the endoplasmic reticulum chaperone BiP. Nature. 2006;443(7111):548-52. Epub 2006/10/07. doi: 10.1038/nature05124. PubMed PMID: 17024087. 
37. Boorstein WR, Ziegelhoffer T, Craig EA. Molecular evolution of the HSP70 multigene family. Journal of molecular evolution. 1994;38(1):1-17. Epub 1994/01/01. PubMed PMID: 8151709.

38. Hamman BD, Hendershot LM, Johnson AE. BiP maintains the permeability barrier of the ER membrane by sealing the lumenal end of the translocon pore before and early in translocation. Cell. 1998;92(6):747-58. Epub 1998/04/07. PubMed PMID: 9529251.

39. Bertolotti A, Zhang Y, Hendershot LM, Harding HP, Ron D. Dynamic interaction of BiP and ER stress transducers in the unfolded-protein response. Nat Cell Biol. 2000;2(6):326-32. Epub 2000/06/15. doi: 10.1038/35014014. PubMed PMID: 10854322 .

40. Lièvremont J-P, Rizzuto R, Hendershot L, Meldolesi J. BiP, a Major Chaperone Protein of the Endoplasmic Reticulum Lumen, Plays a Direct and Important Role in the Storage of the Rapidly Exchanging Pool of Ca2+. Journal of Biological Chemistry. 1997;272(49):30873-9. doi: 10.1074/jbc.272.49.30873.

41. Gaut J, Hendershot L. Mutations within the nucleotide binding site of immunoglobulin-binding protein inhibit ATPase activity and interfere with release of immunoglobulin heavy chain. Journal of Biological Chemistry. 1993;268(10):7248-55.

42. Hendershot LM, Wei JY, Gaut JR, Lawson B, Freiden PJ, Murti KG. In vivo expression of mammalian BiP ATPase mutants causes disruption of the endoplasmic reticulum. Molecular biology of the cell. 1995;6(3):283-96. PubMed PMID: PMC301188.

43. Yang J, Zong Y, Su J, Li H, Zhu H, Columbus L, et al. Conformation transitions of the polypeptide-binding pocket support an active substrate release from Hsp70s. Nature Communications. 2017;8(1):1201. doi: 10.1038/s41467-01701310-z.

44. Behnke J, Hendershot LM. The large Hsp70 Grp170 binds to unfolded protein substrates in vivo with a regulation distinct from conventional Hsp70s. The Journal of biological chemistry. 2014;289(5):2899-907. Epub 2013/12/12. doi: 10.1074/jbc.M1 13.507491. PubMed PMID: 24327659; PubMed Central PMCID: PMCPMC3908422.

45. Yan M, Li J, Sha B. Structural analysis of the Sil1-Bip complex reveals the mechanism for Sil1 to function as a nucleotide-exchange factor. The Biochemical journal. 2011;438(3):447-55. doi: 10.1042/BJ20110500. PubMed PMID: 21675960 .

46. Craven RA, Egerton M, Stirling CJ. A novel Hsp70 of the yeast ER lumen is required for the efficient translocation of a number of protein precursors. The 
EMBO journal. 1996;15(11):2640-50. Epub 1996/06/03. PubMed PMID: 8654361; PubMed Central PMCID: PMCPMC450199.

47. Baxter BK, James P, Evans T, Craig EA. SSI1 encodes a novel Hsp70 of the Saccharomyces cerevisiae endoplasmic reticulum. Molecular and cellular biology. 1996;16(11):6444-56. Epub 1996/11/01. PubMed PMID: 8887673; PubMed Central PMCID: PMCPMC231646.

48. Steel GJ, Fullerton DM, Tyson JR, Stirling CJ. Coordinated activation of Hsp70 chaperones. Science. 2004;303(5654):98-101. Epub 2004/01/06. doi: 10.1126/science.1092287. PubMed PMID: 14704430.

49. Dragovic Z, Broadley SA, Shomura Y, Bracher A, Hartl FU. Molecular chaperones of the Hsp110 family act as nucleotide exchange factors of Hsp70s. The EMBO journal. 2006;25(11):2519-28. Epub 2006/05/12. doi: 10.1038/sj.emboj.7601138. PubMed PMID: 16688212; PubMed Central PMCID: PMCPMC1478182.

50. Weitzmann A, Volkmer J, Zimmermann R. The nucleotide exchange factor activity of Grp170 may explain the non-lethal phenotype of loss of Sill function in man and mouse. FEBS letters. 2006;580(22):5237-40. Epub 2006/09/12. doi: 10.1016/j.febslet.2006.08.055. PubMed PMID: 16962589.

51. Saris N, Holkeri H, Craven RA, Stirling CJ, Makarow M. The Hsp70 homologue Lhslp is involved in a novel function of the yeast endoplasmic reticulum, refolding and stabilization of heat-denatured protein aggregates. J Cell Biol. 1997;137(4):813-24. Epub 1997/05/19. PubMed PMID: 9151684; PubMed Central PMCID: PMCPMC2139846.

52. Hanninen AL, Simola M, Saris N, Makarow M. The cytoplasmic chaperone hsp104 is required for conformational repair of heat-denatured proteins in the yeast endoplasmic reticulum. Molecular biology of the cell. 1999;10(11):3623-32. Epub 1999/11/17. PubMed PMID: 10564260; PubMed Central PMCID: PMCPMC25649.

53. Andreasson C, Rampelt H, Fiaux J, Druffel-Augustin S, Bukau B. The endoplasmic reticulum Grp170 acts as a nucleotide exchange factor of Hsp70 via a mechanism similar to that of the cytosolic Hsp110. The Journal of biological chemistry. 2010;285(16):12445-53. Epub 2010/02/24. doi: 10.1074/jbc.M109.096735. PubMed PMID: 20177057; PubMed Central PMCID: PMCPMC2852982.

54. Buck TM, Plavchak L, Roy A, Donnelly BF, Kashlan OB, Kleyman TR, et al. The Lhs1/GRP170 chaperones facilitate the endoplasmic reticulum-associated degradation of the epithelial sodium channel. The Journal of biological chemistry. 2013;288(25):18366-80. Epub 2013/05/07. doi: 10.1074/jbc.M113.469882. PubMed PMID: 23645669; PubMed Central PMCID: PMCPMC3689978. 
55. Otero JH, Lizak B, Hendershot LM. Life and death of a BiP substrate. Semin Cell Dev Biol. 2010;21(5):472-8. Epub 2009/12/23. doi:

10.1016/j.semcdb.2009.12.008. PubMed PMID: 20026282; PubMed Central PMCID: PMCPMC2883687.

56. Qian L, Tanguay RM, Wu T, Hightower LE. Stress and health Huangshan-style. Cell stress \& chaperones. 2016;21(3):373-8. doi: 10.1007/s12192-016-0674-8. PubMed PMID: PMC4837187.

57. Hendershot L, Wei J, Gaut J, Melnick J, Aviel S, Argon Y. Inhibition of immunoglobulin folding and secretion by dominant negative BiP ATPase mutants. Proceedings of the National Academy of Sciences. 1996;93(11):526974.

58. Blond-Elguindi S, Cwirla SE, Dower WJ, Lipshutz RJ, Sprang SR, Sambrook JF, et al. Affinity panning of a library of peptides displayed on bacteriophages reveals the binding specificity of BiP. Cell. 1993;75(4):717-28. doi: https://doi.org/10.1016/0092-8674(93)90492-9.

59. Behnke J, Mann MJ, Scruggs F-L, Feige MJ, Hendershot LM. Members of the Hsp70 Family Recognize Distinct Types of Sequences to Execute ER Quality Control. Molecular Cell. 2016;63(5):739-52. doi: https://doi.org/10.1016/j.molcel.2016.07.012.

60. Boisramé A, Beckerich J-M, Gaillardin C. Sls1p, an Endoplasmic Reticulum Component, Is Involved in the Protein Translocation Process in the Yeast Yarrowia lipolytica. Journal of Biological Chemistry. 1996;271(20):11668-75. doi: 10.1074/jbc.271.20.11668.

61. Boisramé A, Kabani M, Beckerich J-M, Hartmann E, Gaillardin C. Interaction of Kar2p and Sls1 $p$ Is Required for Efficient Co-translational Translocation of Secreted Proteins in the YeastYarrowia lipolytica. Journal of Biological Chemistry. 1998;273(47):30903-8. doi: 10.1074/jbc.273.47.30903.

62. Tyson JR, Stirling CJ. LHS1 and SIL1 provide a lumenal function that is essential for protein translocation into the endoplasmic reticulum. The EMBO journal. 2000;19(23):6440-52. Epub 2000/12/02. doi: 10.1093/emboj/19.23.6440. PubMed PMID: 11101517; PubMed Central PMCID: PMCPMC305876.

63. Chung KT, Shen Y, Hendershot LM. BAP, a Mammalian BiP-associated Protein, Is a Nucleotide Exchange Factor That Regulates the ATPase Activity of BiP. Journal of Biological Chemistry. 2002;277(49):47557-63. doi: 10.1074/jbc.M208377200.

64. Anttonen AK, Mahjneh I, Hamalainen RH, Lagier-Tourenne C, Kopra O, Waris $\mathrm{L}$, et al. The gene disrupted in Marinesco-Sjogren syndrome encodes SIL1, an HSPA5 cochaperone. Nature genetics. 2005;37(12):1309-11. doi: 10.1038/ng1677. PubMed PMID: 16282978. 
65. Hale SJ, Lovell SC, de Keyzer J, Stirling CJ. Interactions between Kar2p and its nucleotide exchange factors Sillp and Lhs $1 p$ are mechanistically distinct. The Journal of biological chemistry. 2010;285(28):21600-6. Epub 2010/05/01. doi: 10.1074/jbc.M1 10.111211. PubMed PMID: 20430899; PubMed Central PMCID: PMCPMC2898433.

66. Siegenthaler KD, Pareja KA, Wang J, Sevier CS. An unexpected role for the yeast nucleotide exchange factor Sill as a reductant acting on the molecular chaperone BiP. Elife. 2017;6. Epub 2017/03/04. doi: 10.7554/eLife.24141. PubMed PMID: 28257000 ; PubMed Central PMCID: PMCPMC5358974.

67. Wang J, Pareja KA, Kaiser CA, Sevier CS. Redox signaling via the molecular chaperone BiP protects cells against endoplasmic reticulum-derived oxidative stress. Elife. 2014;3:e03496. Epub 2014/07/24. doi: 10.7554/eLife.03496.

PubMed PMID: 25053742; PubMed Central PMCID: PMCPMC4132286.

68. Wang J, Sevier CS. Formation and Reversibility of BiP Protein Cysteine Oxidation Facilitate Cell Survival during and post Oxidative Stress. The Journal of biological chemistry. 2016;291(14):7541-57. Epub 2016/02/13. doi: 10.1074/jbc.M115.694810. PubMed PMID: 26865632; PubMed Central PMCID: PMCPMC4817183.

69. Wang M, Kaufman RJ. The impact of the endoplasmic reticulum protein-folding environment on cancer development. Nature Reviews Cancer. 2014;14:581. doi: $10.1038 / \mathrm{nrc} 3800$ https://www.nature.com/articles/nrc3800\#supplementary-information.

70. Brewer JW, Hendershot LM, Sherr CJ, Diehl JA. Mammalian unfolded protein response inhibits cyclin D1 translation and cell-cycle progression. Proceedings of the National Academy of Sciences of the United States of America. 1999;96(15):8505-10. Epub 1999/07/21. PubMed PMID: 10411905; PubMed Central PMCID: PMCPMC17546.

71. Gardner BM, Walter P. Unfolded proteins are Ire1-activating ligands that directly induce the unfolded protein response. Science. 2011;333(6051):1891-4. Epub 2011/08/20. doi: 10.1126/science.1209126. PubMed PMID: 21852455; PubMed Central PMCID: PMCPMC3202989.

72. Shaffer AL, Shapiro-Shelef M, Iwakoshi NN, Lee A-H, Qian S-B, Zhao H, et al. XBP1, Downstream of Blimp-1, Expands the Secretory Apparatus and Other Organelles, and Increases Protein Synthesis in Plasma Cell Differentiation. Immunity. 2004;21(1):81-93. doi: https://doi.org/10.1016/j.immuni.2004.06.010.

73. Walter P, Ron D. The Unfolded Protein Response: From Stress Pathway to Homeostatic Regulation. Science. 2011;334(6059):1081-6. doi:

10.1126/science. 1209038 . 
74. Shen J, Snapp EL, Lippincott-Schwartz J, Prywes R. Stable binding of ATF6 to $\mathrm{BiP}$ in the endoplasmic reticulum stress response. Molecular and cellular biology. 2005;25(3):921-32. Epub 2005/01/20. doi: 10.1128/mcb.25.3.921-932.2005. PubMed PMID: 15657421; PubMed Central PMCID: PMCPMC543992.

75. Marinesco G. Novelle maladie familiale caracterisee pare une cataracte congenitale et un arret du development somato-neurophysique. L'Encephale. 1931;26:97-109.

76. Sjogren T. Hereditary congenital spinocerebellar ataxia accompanied by congenital cataract and oligophrenia; a genetic and clinical investigation. Confinia neurologica. 1950;10(5):293-308. Epub 1950/01/01. PubMed PMID: 14792949.

77. Alter M, Talbert OR, Croffead G. Cerebellar ataxia, congenital cataracts, and retarded somatic and mental maturation. Report of cases of Marinesco-Sjogren syndrome. Neurology. 1962;12:836-47. PubMed PMID: 14012309.

78. Krieger M, Roos A, Stendel C, Claeys KG, Sonmez FM, Baudis M, et al. SIL1 mutations and clinical spectrum in patients with Marinesco-Sjögren syndrome2013 2013-10-30 00:00:00.

79. Superneau DW, Wertelecki W, Zellweger H, Bastian F. Myopathy in MarinescoSjogren syndrome. European neurology. 1987;26(1):8-16. PubMed PMID: 3469098 .

80. Sewry CA, Voit T, Dubowitz V. Myopathy with unique ultrastructural feature in Marinesco-Sjogren syndrome. Annals of neurology. 1988;24(4):576-80. doi: 10.1002/ana.410240416. PubMed PMID: 3239958.

81. Goto Y, Komiyama A, Tanabe Y, Katafuchi Y, Ohtaki E, Nonaka I. Myopathy in Marinesco-Sjogren syndrome: an ultrastructural study. Acta neuropathologica. 1990;80(2):123-8. PubMed PMID: 2389676.

82. Sasaki K, Suga K, Tsugawa S, Sakuma K, Tachi N, Chiba S, et al. Muscle pathology in Marinesco-Sjogren syndrome: a unique ultrastructural feature. Brain \& development. 1996;18(1):64-7. PubMed PMID: 8907346.

83. Mahjneh I, Anttonen AK, Somer M, Paetau A, Lehesjoki AE, Somer H, et al. Myopathy is a prominent feature in Marinesco-Sjogren syndrome: A muscle computed tomography study. Journal of neurology. 2006;253(3):301-6. doi: 10.1007/s00415-005-0983-9. PubMed PMID: 16151599.

84. Torbergsen T, Stalberg E, Aasly J, Lindal S. Myopathy in Marinesco-Sjogren syndrome: an electrophysiological study. Acta neurologica Scandinavica. 1991;84(2):132-8. Epub 1991/08/01. PubMed PMID: 1659103.

85. Lagier-Tourenne C, Tranebaerg L, Chaigne D, Gribaa M, Dollfus H, Silvestri G, et al. Homozygosity mapping of Marinesco-Sjogren syndrome to 5q31. European 
journal of human genetics : EJHG. 2003;11(10):770-8. Epub 2003/09/27. doi: 10.1038/sj.ejhg.5201068. PubMed PMID: 14512967.

86. Senderek J, Krieger M, Stendel C, Bergmann C, Moser M, Breitbach-Faller N, et al. Mutations in SIL1 cause Marinesco-Sjogren syndrome, a cerebellar ataxia with cataract and myopathy. Nature genetics. 2005;37(12):1312-4. doi:

10.1038/ng1678. PubMed PMID: 16282977.

87. Zhao L, Longo-Guess C, Harris BS, Lee JW, Ackerman SL. Protein accumulation and neurodegeneration in the woozy mutant mouse is caused by disruption of SIL1, a cochaperone of BiP. Nature genetics. 2005;37(9):974-9. doi: 10.1038/ng1620. PubMed PMID: 16116427.

88. Anttonen AK, Siintola E, Tranebjaerg L, Iwata NK, Bijlsma EK, Meguro H, et al. Novel SIL1 mutations and exclusion of functional candidate genes in MarinescoSjogren syndrome. European journal of human genetics : EJHG. 2008;16(8):9619. doi: 10.1038/ejhg.2008.22. PubMed PMID: 18285827.

89. Howes J, Shimizu Y, Feige MJ, Hendershot LM. C-terminal mutations destabilize SIL1/BAP and can cause Marinesco-Sjogren syndrome. The Journal of biological chemistry. 2012;287(11):8552-60. Epub 2012/01/06. doi:

10.1074/jbc.M111.333286. PubMed PMID: 22219183; PubMed Central PMCID: PMCPmc3318681.

90. Inaguma Y, Hamada N, Tabata H, Iwamoto I, Mizuno M, Nishimura YV, et al. SIL1, a causative cochaperone gene of Marinesco-Sojgren syndrome, plays an essential role in establishing the architecture of the developing cerebral cortex. EMBO molecular medicine. 2014;6(3):414-29. Epub 2014/01/30. doi: 10.1002/emmm.201303069. PubMed PMID: 24473200; PubMed Central PMCID: PMCPmc3958314.

91. Zhao L, Rosales C, Seburn K, Ron D, Ackerman SL. Alteration of the unfolded protein response modifies neurodegeneration in a mouse model of MarinescoSjogren syndrome. Human molecular genetics. 2010;19(1):25-35. doi: 10.1093/hmg/ddp464. PubMed PMID: 19801575; PubMed Central PMCID: PMC2792147.

92. Ellgaard L, Helenius A. Quality control in the endoplasmic reticulum. Nature reviews Molecular cell biology. 2003;4(3):181-91.

93. Marcinowski M, Höller M, Feige MJ, Baerend D, Lamb DC, Buchner J. Substrate discrimination of the chaperone $\mathrm{BiP}$ by autonomous and cochaperone-regulated conformational transitions. Nature structural \& molecular biology. 2011;18(2):150-8.

94. Marcinowski M, Rosam M, Seitz C, Elferich J, Behnke J, Bello C, et al. Conformational selection in substrate recognition by Hsp70 chaperones. Journal of molecular biology. 2013;425(3):466-74. 
95. Wei X, Pan S, Lang W, Kim H, Schneider T, Perez-Reyes E, et al. Molecular determinants of cardiac $\mathrm{Ca} 2+$ channel pharmacology. Subunit requirement for the high affinity and allosteric regulation of dihydropyridine binding. The Journal of biological chemistry. 1995;270(45):27106-11.

96. Kabani M, Beckerich JM, Gaillardin C. Sls1p stimulates Sec63p-mediated activation of Kar2p in a conformation-dependent manner in the yeast endoplasmic reticulum. Molecular and cellular biology. 2000;20(18):6923-34.

97. Lin HY, Masso-Welch P, Di YP, Cai JW, Shen JW, Subjeck JR. The 170-kDa glucose-regulated stress protein is an endoplasmic reticulum protein that binds immunoglobulin. Molecular biology of the cell. 1993;4(11):1109-19.

98. Spee P, Subjeck J, Neefjes J. Identification of novel peptide binding proteins in the endoplasmic reticulum: ERp72, calnexin, and grp170. Biochemistry. 1999;38(32):10559-66.

99. Horvers M, Anttonen AK, Lehesjoki AE, Morava E, Wortmann S, Vermeer S, et al. Marinesco-Sjogren syndrome due to SIL1 mutations with a comment on the clinical phenotype. European journal of paediatric neurology : EJPN : official journal of the European Paediatric Neurology Society. 2013;17(2):199-203. Epub 2012/10/16. doi: 10.1016/j.ejpn.2012.09.007. PubMed PMID: 23062754.

100. Roos A, Buchkremer S, Kollipara L, Labisch T, Gatz C, Zitzelsberger M, et al. Myopathy in Marinesco-Sjogren syndrome links endoplasmic reticulum chaperone dysfunction to nuclear envelope pathology. Acta neuropathologica. 2014;127(5):761-77. Epub 2013/12/24. doi: 10.1007/s00401-013-1224-4. PubMed PMID: 24362440.

101. Feige MJ, Groscurth S, Marcinowski M, Shimizu Y, Kessler H, Hendershot LM, et al. An unfolded $\mathrm{CH} 1$ domain controls the assembly and secretion of $\operatorname{IgG}$ antibodies. Molecular cell. 2009;34(5):569-79.

102. Hendershot L, Bole D, Köhler G, Kearney JF. Assembly and secretion of heavy chains that do not associate posttranslationally with immunoglobulin heavy chainbinding protein. The Journal of cell biology. 1987;104(3):761-7.

103. Lee LS, Conover C, Shi C, Whitlow M, Filpula D. Prolonged circulating lives of single-chain Fv proteins conjugated with polyethylene glycol: a comparison of conjugation chemistries and compounds. Bioconjugate chemistry. 1999;10(6):973-81.

104. Vanhove M, Usherwood YK, Hendershot LM. Unassembled Ig heavy chains do not cycle from $\mathrm{BiP}$ in vivo but require light chains to trigger their release. Immunity. 2001;15(1):105-14.

105. Ittner AA, Bertz J, Chan TY, van Eersel J, Polly P, Ittner LM. The nucleotide exchange factor SIL1 is required for glucose-stimulated insulin secretion from 
mouse pancreatic beta cells in vivo. Diabetologia. 2014;57(7):1410-9. Epub 2014/04/16. doi: 10.1007/s00125-014-3230-z. PubMed PMID: 24733160.

106. Wiest DL, Burkhardt JK, Hester S, Hortsch M, Meyer DI, Argon Y. Membrane biogenesis during $\mathrm{B}$ cell differentiation: most endoplasmic reticulum proteins are expressed coordinately. The Journal of cell biology. 1990;110(5):1501-11.

107. van Anken E, Romijn EP, Maggioni C, Mezghrani A, Sitia R, Braakman I, et al. Sequential waves of functionally related proteins are expressed when B cells prepare for antibody secretion. Immunity. 2003;18(2):243-53. Epub 2003/02/22. PubMed PMID: 12594951.

108. Gass JN, Gifford NM, Brewer JW. Activation of an unfolded protein response during differentiation of antibody-secreting B cells. The Journal of biological chemistry. 2002;277(50):49047-54.

109. Iwakoshi NN, Lee A-H, Vallabhajosyula P, Otipoby KL, Rajewsky K, Glimcher LH. Plasma cell differentiation and the unfolded protein response intersect at the transcription factor XBP-1. Nature immunology. 2003;4(4):321-9.

110. Park J, Easton DP, Chen X, MacDonald IJ, Wang XY, Subjeck JR. The chaperoning properties of mouse grp170, a member of the third family of hsp70 related proteins. Biochemistry. 2003;42(50):14893-902. doi: 10.1021/bi030122e. PubMed PMID: 14674765.

111. David V, Hochstenbach F, Rajagopalan S, Brenner MB. Interaction with newly synthesized and retained proteins in the endoplasmic reticulum suggests a chaperone function for human integral membrane protein IP90 (calnexin). The Journal of biological chemistry. 1993;268(13):9585-92.

112. Melnick J, Aviel S, Argon Y. The endoplasmic reticulum stress protein GRP94, in addition to $\mathrm{BiP}$, associates with unassembled immunoglobulin chains. Journal of Biological Chemistry. 1992;267(30):21303-6.

113. Shen Y, Hendershot LM. ERdj3, a stress-inducible endoplasmic reticulum DnaJ homologue, serves as a cofactor for BiP's interactions with unfolded substrates. Molecular biology of the cell. 2005;16(1):40-50. doi: 10.1091/mbc.E04-05-0434. PubMed PMID: 15525676; PubMed Central PMCID: PMC539150.

114. Conley ME, Rohrer J, Rapalus L, Boylin EC, Minegishi Y. Defects in early B-cell development: comparing the consequences of abnormalities in pre-BCR signaling in the human and the mouse. Immunological reviews. 2000;178:75-90.

115. Ezgu F, Krejci P, Li S, de Sousa C, Graham JM, Jr., Hansmann I, et al. Phenotype-genotype correlations in patients with Marinesco-Sjogren syndrome. Clinical genetics. 2014;86(1):74-84. Epub 2013/07/09. doi: 10.1111/cge.12230. PubMed PMID: 23829326. 
116. Barends TRM, Brosi RWW, Steinmetz A, Scherer A, Hartmann E, Eschenbach J, et al. Combining crystallography and EPR: crystal and solution structures of the multidomain cochaperone DnaJ. Acta crystallographica Section D, Biological crystallography. 2013;69(Pt 8):1540-52.

117. Superneau DW, Wertelecki W, Zellweger H, Bastian F. Myopathy in MarinescoSjogren syndrome. European neurology. 1987;26(1):8-16.

118. Hasegawa S, Imai K, Yoshida K, Okuno Y, Muramatsu H, Shiraishi Y, et al. Whole-exome sequence analysis of ataxia telangiectasia-like phenotype. Journal of the neurological sciences. 2014;340(1-2):86-90.

119. Abrams JL, Verghese J, Gibney PA, Morano KA. Hierarchical functional specificity of cytosolic heat shock protein 70 (Hsp70) nucleotide exchange factors in yeast. The Journal of biological chemistry. 2014;289(19):13155-67. Epub 2014/03/29. doi: 10.1074/jbc.M113.530014. PubMed PMID: 24671421; PubMed Central PMCID: PMCPMC4036327.

120. Hohfeld J, Jentsch S. GrpE-like regulation of the hsc70 chaperone by the antiapoptotic protein BAG-1. The EMBO journal. 1997;16:6209-16.

121. Ben-Zvi A, Miller EA, Morimoto RI. Collapse of proteostasis represents an early molecular event in Caenorhabditis elegans aging. Proceedings of the National Academy of Sciences of the United States of America. 2009;106(35):14914-9.

122. Moore GJ, Aldred P. Treatment of Pasteurella pneumotropica abscesses in nude mice (nu/nu). Laboratory animals. 1978;12(4):227-8. Epub 1978/10/01. doi: 10.1258/002367778781088413. PubMed PMID: 732265.

123. Okuda-Shimizu Y, Hendershot LM. Characterization of an ERAD Pathway for Nonglycosylated BiP Substrates, which Require Herp. Molecular Cell. 2007;28(4):544-54. doi: https://doi.org/10.1016/j.molcel.2007.09.012.

124. Lawson B, Brewer JW, Hendershot LM. Geldanamycin, an hsp90/GRP94-binding drug, induces increased transcription of endoplasmic reticulum (ER) chaperones via the ER stress pathway. Journal of cellular physiology. 1998;174(2):170-8.

125. Ahner A, Brodsky JL. Checkpoints in ER-associated degradation: excuse me, which way to the proteasome? Trends in cell biology. 2004;14(9):474-8.

126. Qi L, Tsai B, Arvan P. New Insights into the Physiological Role of Endoplasmic Reticulum-Associated Degradation. Trends in cell biology. 2017;27(6):430-40.

127. McCaffrey K, Braakman I. Protein quality control at the endoplasmic reticulum. Essays in biochemistry. 2016;60(2):227-35. 
128. Wei J, Gaut JR, Hendershot LM. In vitro dissociation of BiP-peptide complexes requires a conformational change in $\mathrm{BiP}$ after $\mathrm{ATP}$ binding but does not require ATP hydrolysis. Journal of Biological Chemistry. 1995;270(44):26677-82.

129. Williams WM, Berry DC. A Qualitative Study: African-American Girls' Perceptions of Why Physical Activity Declines in High School. Journal of National Black Nurses' Association : JNBNA. 2015;26(2):60-6.

130. Inoue T, Dosey A, Herbstman JF, Ravindran MS, Skiniotis G, Tsai B. ERdj5 Reductase Cooperates with Protein Disulfide Isomerase To Promote Simian Virus 40 Endoplasmic Reticulum Membrane Translocation. Journal of virology. 2015;89(17):8897-908.

131. Inoue D, Tsunoda T, Sawada K, Yamamoto N, Saito Y, Sei K, et al. 1,4-Dioxane degradation potential of members of the genera Pseudonocardia and Rhodococcus. Biodegradation. 2016;27(4-6):277-86.

132. Kitao Y, Ozawa K, Miyazaki M, Tamatani M, Kobayashi T, Yanagi H, et al. Expression of the endoplasmic reticulum molecular chaperone (ORP150) rescues hippocampal neurons from glutamate toxicity. The Journal of clinical investigation. 2001;108(10):1439-50.

133. Ichhaporia VP, Sanford T, Howes J, Marion TN, Hendershot LM. Sil a nucleotide exchange factor for BiP, is not required for antibody assembly or secretion. MolBiolCell available from PM25473114. 2014;1 SRC - BaiduScholar.

134. Weitzmann A, Baldes C, Dudek J, Zimmermann R. The heat shock protein 70 molecular chaperone network in the pancreatic endoplasmic reticulum - a quantitative approach. The FEBS journal. 2007;274(19):5175-87.

135. Filézac de L'Etang A, Maharjan N, Cordeiro Braña M, Ruegsegger C, Rehmann R, Goswami A, et al. Marinesco-Sjögren syndrome protein SIL1 regulates motor neuron subtype-selective ER stress in ALS. Nature neuroscience. 2015;18:227. doi: 10.1038/nn.3903 https://www.nature.com/articles/nn.3903\#supplementary-information.

136. Stelzer G, Rosen N, Plaschkes I, Zimmerman S, Twik M, Fishilevich S, et al. The GeneCards Suite: From Gene Data Mining to Disease Genome Sequence Analyses. Current protocols in bioinformatics. 2016;54:1.30.1-1..3.

137. Demontis F, Piccirillo R, Goldberg AL, Perrimon N. Mechanisms of skeletal muscle aging: insights from $<\mathrm{em}>$ Drosophila $</ \mathrm{em}>$ and mammalian models. Disease models \& mechanisms. 2013;6(6):1339-52. doi: 10.1242/dmm.012559.

138. Lopez-Otin C, Blasco MA, Partridge L, Serrano M, Kroemer G. The hallmarks of aging. Cell 6 available from PM23746838. 2013;153 SRC - BaiduScholar:1194217. 
139. Hartwig S, Raschke S, Knebel B, Scheler M, Irmler M, Passlack W, et al. Secretome profiling of primary human skeletal muscle cells. Biochimica et biophysica acta. 2014;1844(5):1011-7.

140. Mirabella M, Alvarez RB, Engel WK, Weisgraber KH, Askanas V. Apolipoprotein E and apolipoprotein E messenger RNA in muscle of inclusion body myositis and myopathies. Annals of neurology. 1996;40(6):864-72.

141. Sardiello M, Palmieri M, di Ronza A, Medina DL, Valenza M, Gennarino VA, et al. A gene network regulating lysosomal biogenesis and function. Science (New York, NY). 2009;325(5939):473-7.

142. Mansueto G, Armani A, Viscomi C, D'Orsi L, De Cegli R, Polishchuk EV, et al. Transcription Factor EB Controls Metabolic Flexibility during Exercise. Cell metabolism. 2017;25(1):182-96.

143. Dubinska-Magiera M, Jablonska J, Saczko J, Kulbacka J, Jagla T, Daczewska M, et al. Contribution of small heat shock proteins to muscle development and function. 4 available from PM24440355. 2014;588 SRC - BaiduScholar:517-30.

144. Arrigo A-P, Simon S, Gibert B, Kretz-Remy C, Nivon M, Czekalla A, et al. Hsp27 (HspB1) and alphaB-crystallin (HspB5) as therapeutic targets. FEBS letters. 2007;581(19):3665-74.

145. Ramalingam L, Oh E, Thurmond DC. Novel roles for insulin receptor (IR) in adipocytes and skeletal muscle cells via new and unexpected substrates. Cellular and molecular life sciences : CMLS. 2013;70(16):2815-34.

146. Schiaffino S, Dyar KA, Ciciliot S, Blaauw B, Sandri M. Mechanisms regulating skeletal muscle growth and atrophy. The FEBS journal. 2013;280(17):4294-314.

147. Gingras AC, Raught B, Sonenberg N. mTOR signaling to translation. Current topics in microbiology and immunology. 2004;279:169-97.

148. Kim KH, Lee M-S. FGF21 as a mediator of adaptive responses to stress and metabolic benefits of anti-diabetic drugs. The Journal of endocrinology. 2015;226(1):R1-16.

149. Ebert SM, Dyle MC, Kunkel SD, Bullard SA, Bongers KS, Fox DK, et al. Stressinduced skeletal muscle Gadd45a expression reprograms myonuclei and causes muscle atrophy. The Journal of biological chemistry. 2012;287(33):27290-301.

150. Rowland AF, Fazakerley DJ, James DE. Mapping insulin/GLUT4 circuitry. Traffic (Copenhagen, Denmark). 2011;12(6):672-81.

151. Acosta-Alvear D, Zhou Y, Blais A, Tsikitis M, Lents NH, Arias C, et al. XBP1 controls diverse cell type- and condition-specific transcriptional regulatory networks. Molecular cell. 2007;27(1):53-66. 
152. Iwawaki T, Akai R, Kohno K, Miura M. A transgenic mouse model for monitoring endoplasmic reticulum stress. Nature medicine. 2004;10(1):98-102.

153. Munoz JP, Ivanova S, Sanchez-Wandelmer J, Martinez-Cristobal P, Noguera E, Sancho A, et al. Mfn2 modulates the UPR and mitochondrial function via repression of PERK. EMBO J 17 available from PM23921556. 2013;32 SRC BaiduScholar:2348-61.

154. Blais A, Tsikitis M, Acosta-Alvear D, Sharan R, Kluger Y, Dynlacht BD. An initial blueprint for myogenic differentiation. Genes \& development. 2005;19(5):553-69.

155. Wu J, Ruas JL, Estall JL, Rasbach KA, Choi JH. The Unfolded Protein Response Mediates Adaptation to Exercise in Skeletal Muscle through a PGC-1 $\alpha$ /ATF6 $\alpha$ Complex. Cell metabolism. 2011;13(2).

156. O'Neill Brian T, Lauritzen Hans PMM, Hirshman Michael F, Smyth G, Goodyear Laurie J, Kahn CR. Differential Role of Insulin/IGF-1 Receptor Signaling in Muscle Growth and Glucose Homeostasis. Cell reports. 2015;11(8):1220-35. doi: https://doi.org/10.1016/j.celrep.2015.04.037.

157. Ozcan U, Cao Q, Yilmaz E, Lee A-H, Iwakoshi NN, Ozdelen E, et al. Endoplasmic reticulum stress links obesity, insulin action, and type 2 diabetes. Science (New York, NY). 2004;306(5695):457-61.

158. Glass DJ. PI3 Kinase Regulation of Skeletal Muscle Hypertrophy and Atrophy. In: Rommel C, Vanhaesebroeck B, Vogt PK, editors. Phosphoinositide 3-kinase in Health and Disease: Volume 1. Berlin, Heidelberg: Springer Berlin Heidelberg; 2011. p. 267-78.

159. Laplante M, Sabatini DM. mTOR signaling in growth control and disease. Cell. 2012;149(2):274-93.

160. Kim J, Kundu M, Viollet B, Guan KL. AMPK and mTOR regulate autophagy through direct phosphorylation of Ulk1. NatCell Biol 2 available from PM21258367. 2011;13 SRC - BaiduScholar:132-41.

161. Cohen M, Dawson MS, Kopistansky C, McBride R. Sex and other predictors of intra-aortic balloon counterpulsation-related complications: prospective study of 1119 consecutive patients. American heart journal. 2000;139(2 Pt 1):282-7.

162. Morley S, Williams ACdC, Black S. A confirmatory factor analysis of the Beck Depression Inventory in chronic pain. Pain. 2002;99(1-2):289-98.

163. Tain LS, Mortiboys H, Tao RN, Ziviani E, Bandmann O, Whitworth AJ. Rapamycin activation of 4E-BP prevents parkinsonian dopaminergic neuron loss. Nature neuroscience. 2009;12(9):1129-35. 
164. Spilman P, Podlutskaya N, Hart MJ, Debnath J, Gorostiza O, Bredesen D, et al. Inhibition of mTOR by rapamycin abolishes cognitive deficits and reduces amyloid-beta levels in a mouse model of Alzheimer's disease. PloS one. 2010;5(4):e9979.

165. Taylor JP, Hardy J, Fischbeck KH. Toxic proteins in neurodegenerative disease. Science (New York, NY). 2002;296(5575):1991-5.

166. Taylor RC, Dillin A. Aging as an event of proteostasis collapse. Cold Spring Harbor perspectives in biology. 2011;3(5).

167. Powers ET, Morimoto RI, Dillin A, Kelly JW, Balch WE. Biological and chemical approaches to diseases of proteostasis deficiency. Annual review of biochemistry. 2009;78:959-91. Epub 2009/03/21. doi:

10.1146/annurev.biochem.052308.114844. PubMed PMID: 19298183.

168. Deacon RMJ. Measuring the strength of mice. Journal of visualized experiments : JoVE. 2013(76).

169. Bai B, Tan H, Pagala VR, High AA, Ichhaporia VP, Hendershot L, et al. Deep Profiling of Proteome and Phosphoproteome by Isobaric Labeling, Extensive Liquid Chromatography, and Mass Spectrometry. Methods in enzymology. 2017;585:377-95.

170. Wang X, Li Y, Wu Z, Wang H, Tan H, Peng J. JUMP: a tag-based database search tool for peptide identification with high sensitivity and accuracy. Molecular \& cellular proteomics : MCP. 2014;13(12):3663-73.

171. Thomas LC, Rivett DA, Parsons M, Levi C. Risk factors, radiological features, and infarct topography of craniocervical arterial dissection. International journal of stroke : official journal of the International Stroke Society. 2014;9(8):1073-82.

172. Shomura Y, Dragovic Z, Chang HC, Tzvetkov N, Young JC, Brodsky JL, et al. Regulation of Hsp70 function by HspBP1: structural analysis reveals an alternate mechanism for Hsp70 nucleotide exchange. Mol Cell. 2005;17(3):367-79. Epub 2005/02/08. doi: 10.1016/j.molcel.2004.12.023. PubMed PMID: 15694338.

173. Riazuddin SA, Amiri-Kordestani L, Kaul H, Butt T, Jiao X, Riazuddin S, et al. Novel SIL1 mutations in consanguineous Pakistani families mapping to chromosomes 5q31. Mol Vis. 2009;15:1050-6. Epub 2009/05/28. PubMed PMID: 19471582; PubMed Central PMCID: PMCPMC2685889.

174. Goto M, Okada M, Komaki H, Sugai K, Sasaki M, Noguchi S, et al. A nationwide survey on Marinesco-Sjogren syndrome in Japan. Orphanet journal of rare diseases. 2014;9:58. Epub 2014/04/24. doi: 10.1186/1750-1172-9-58. PubMed PMID: 24755310; PubMed Central PMCID: PMCPMC4021608. 
175. Sievers F, Wilm A, Dineen D, Gibson TJ, Karplus K, Li W, et al. Fast, scalable generation of high-quality protein multiple sequence alignments using Clustal Omega. Molecular systems biology. 2011;7:539. Epub 2011/10/13. doi: 10.1038/msb.2011.75. PubMed PMID: 21988835; PubMed Central PMCID: PMCPMC3261699.

176. Hecht M, Bromberg Y, Rost B. Better prediction of functional effects for sequence variants. BMC genomics. 2015;16 Suppl 8:S1. Epub 2015/06/26. doi: 10.1186/1471-2164-16-s8-s1. PubMed PMID: 26110438; PubMed Central PMCID: PMCPMC4480835.

177. Fernandez-Escamilla AM, Rousseau F, Schymkowitz J, Serrano L. Prediction of sequence-dependent and mutational effects on the aggregation of peptides and proteins. Nature biotechnology. 2004;22(10):1302-6. Epub 2004/09/14. doi: 10.1038/nbt1012. PubMed PMID: 15361882.

178. Abrusán G, Marsh JA. Alpha Helices Are More Robust to Mutations than Beta Strands. PLOS Computational Biology. 2016;12(12):e1005242. doi: 10.1371/journal.pcbi.1005242.

179. Valastyan JS, Lindquist S. Mechanisms of protein-folding diseases at a glance. Disease models \& mechanisms. 2014;7(1):9-14. Epub 2014/01/08. doi: 10.1242/dmm.013474. PubMed PMID: 24396149; PubMed Central PMCID: PMCPMC3882043.

180. Cortez L, Sim V. The therapeutic potential of chemical chaperones in protein folding diseases. Prion. 2014;8(2). Epub 2014/05/14. PubMed PMID: 24818993; PubMed Central PMCID: PMCPMC4189890.

181. Kerem E. Pharmacological induction of CFTR function in patients with cystic fibrosis: mutation-specific therapy. Pediatric pulmonology. 2005;40(3):183-96. Epub 2005/05/10. doi: 10.1002/ppul.20200. PubMed PMID: 15880796.

182. Lim M, McKenzie K, Floyd AD, Kwon E, Zeitlin PL. Modulation of deltaF508 cystic fibrosis transmembrane regulator trafficking and function with 4phenylbutyrate and flavonoids. American journal of respiratory cell and molecular biology. 2004;31(3):351-7. Epub 2004/06/12. doi: 10.1165/rcmb.2002-0086OC. PubMed PMID: 15191910.

183. Burrows JA, Willis LK, Perlmutter DH. Chemical chaperones mediate increased secretion of mutant alpha 1-antitrypsin (alpha 1-AT) Z: A potential pharmacological strategy for prevention of liver injury and emphysema in alpha 1-AT deficiency. Proceedings of the National Academy of Sciences of the United States of America. 2000;97(4):1796-801. Epub 2000/03/04. PubMed PMID: 10677536; PubMed Central PMCID: PMCPMC26515.

184. Fujiwara M, Yamamoto H, Miyagi T, Seki T, Tanaka S, Hide I, et al. Effects of the chemical chaperone 4-phenylbutylate on the function of the serotonin 
transporter (SERT) expressed in COS-7 cells. Journal of pharmacological sciences. 2013;122(2):71-83. Epub 2013/05/17. PubMed PMID: 23676312.

185. Ma L, Liu Y, El-Achkar TM, Wu XR. Molecular and cellular effects of TammHorsfall protein mutations and their rescue by chemical chaperones. The Journal of biological chemistry. 2012;287(2):1290-305. Epub 2011/11/26. doi: 10.1074/jbc.M111.283036. PubMed PMID: 22117067; PubMed Central PMCID: PMCPMC3256885.

186. Liu XL, Done SC, Yan K, Kilpelainen P, Pikkarainen T, Tryggvason K. Defective trafficking of nephrin missense mutants rescued by a chemical chaperone. Journal of the American Society of Nephrology : JASN. 2004;15(7):1731-8. Epub 2004/06/24. PubMed PMID: 15213260.

187. Winter L, Staszewska I, Mihailovska E, Fischer I, Goldmann WH, Schroder R, et al. Chemical chaperone ameliorates pathological protein aggregation in plectindeficient muscle. The Journal of clinical investigation. 2014;124(3):1144-57. Epub 2014/02/04. doi: 10.1172/jci71919. PubMed PMID: 24487589; PubMed Central PMCID: PMCPMC3934181.

188. Chen YM, Zhou Y, Go G, Marmerstein JT, Kikkawa Y, Miner JH. Laminin beta2 gene missense mutation produces endoplasmic reticulum stress in podocytes. Journal of the American Society of Nephrology : JASN. 2013;24(8):1223-33. Epub 2013/06/01. doi: 10.1681/asn.2012121149. PubMed PMID: 23723427; PubMed Central PMCID: PMCPMC3736718.

189. Zhang YJ, Jansen-West K, Xu YF, Gendron TF, Bieniek KF, Lin WL, et al. Aggregation-prone c9FTD/ALS poly(GA) RAN-translated proteins cause neurotoxicity by inducing ER stress. Acta neuropathologica. 2014;128(4):505-24. Epub 2014/09/01. doi: 10.1007/s00401-014-1336-5. PubMed PMID: 25173361; PubMed Central PMCID: PMCPMC4159567.

190. Kabani M, Beckerich JM, Gaillardin C. Sls1p stimulates Sec63p-mediated activation of Kar2p in a conformation-dependent manner in the yeast endoplasmic reticulum. Molecular and cellular biology. 2000;20(18):6923-34. Epub 2000/08/25. PubMed PMID: 10958688; PubMed Central PMCID: PMCPMC88768.

191. Petrova K, Oyadomari S, Hendershot LM, Ron D. Regulated association of misfolded endoplasmic reticulum lumenal proteins with P58/DNAJc3. The EMBO journal. 2008;27(21):2862-72. Epub 2008/10/17. doi: 10.1038/emboj.2008.199. PubMed PMID: 18923430; PubMed Central PMCID: PMCPMC2580781.

192. Yang J, Nune M, Zong Y, Zhou L, Liu Q. Close and Allosteric Opening of the Polypeptide-Binding Site in a Human Hsp70 Chaperone BiP. Structure. 2015;23(12):2191-203. doi: https://doi.org/10.1016/j.str.2015.10.012. 
193. Jin Y, Awad W, Petrova K, Hendershot LM. Regulated release of ERdj3 from unfolded proteins by BiP. The EMBO journal. 2008;27(21):2873-82. Epub 2008/10/17. doi: 10.1038/emboj.2008.207. PubMed PMID: 18923428; PubMed Central PMCID: PMCPMC2580786.

194. Ichhaporia VP, Kim J, Kavdia K, Vogel P, Horner L, Frase S, et al. SIL1, the endoplasmic-reticulum-localized BiP co-chaperone, plays a crucial role in maintaining skeletal muscle proteostasis and physiology. Disease models \& mechanisms. 2018;11(5). doi: 10.1242/dmm.033043.

195. Yiş U, Cirak S, Hız S, Çakmakçı H, Dirik E. Heterogeneity of Marinesco-Sjögren Syndrome: Report of Two Cases. Pediatric neurology. 2011;45(6):409-11. doi: https://doi.org/10.1016/j.pediatrneurol.2011.08.015.

196. Synofzik M, Haack Tobias B, Kopajtich R, Gorza M, Rapaport D, Greiner M, et al. Absence of BiP Co-chaperone DNAJC3 Causes Diabetes Mellitus and Multisystemic Neurodegeneration. The American Journal of Human Genetics. 2014;95(6):689-97. doi: https://doi.org/10.1016/j.ajhg.2014.10.013.

197. Ladiges WC, Knoblaugh SE, Morton JF, Korth MJ, Sopher BL, Baskin CR, et al. Pancreatic $\beta$-Cell Failure and Diabetes in Mice With a Deletion Mutation of the Endoplasmic Reticulum Molecular Chaperone Gene P58<sup $>$ IPK $</$ sup $>$. Diabetes. 2005;54(4):1074-81. doi: 10.2337/diabetes.54.4.1074.

198. Deane S. Significance of Glucose-Regulated Protein GRP170 (ORP150) in Mouse Striatal Development.

199. Wiessner M, Roos A, Munn CJ, Viswanathan R, Whyte T, Cox D, et al. Mutations in INPP5K, Encoding a Phosphoinositide 5-Phosphatase, Cause Congenital Muscular Dystrophy with Cataracts and Mild Cognitive Impairment. The American Journal of Human Genetics. 2017;100(3):523-36. doi: https://doi.org/10.1016/j.ajhg.2017.01.024.

200. Kitao Y, Hashimoto K, Matsuyama T, Iso H, Tamatani T, Hori O, et al. ORP150/HSP12A Regulates Purkinje Cell Survival: A Role for Endoplasmic Reticulum Stress in Cerebellar Development. The Journal of Neuroscience. 2004;24(6):1486-96. doi: 10.1523/jneurosci.4029-03.2004.

201. Kobayashi T, Takita Y, Suzuki A, Katsu Y, Iguchi T, Ohta Y. Vacuolar Degeneration of Skeletal Muscle in Transgenic Mice Overexpressing ORP150. Journal of Veterinary Medical Science. 2008;70(1):115-8. doi: 10.1292/jvms.70.115.

202. Mimura N, Yuasa S, Soma M, Jin H, Kimura K, Goto S, et al. Altered Quality Control in the Endoplasmic Reticulum Causes Cortical Dysplasia in Knock-In Mice Expressing a Mutant BiP. Molecular and cellular biology. 2008;28(1):293301. doi: 10.1128/mcb.00473-07. 
203. Komiyama A, Nonaka I, Hirayama K. Muscle pathology in Marinesco-Sjogren syndrome. Journal of the neurological sciences. 1989;89(1):103-13. PubMed PMID: 2522540.

204. Zimmer C, Gosztonyi G, Cervos-Navarro J, von Moers A, Schroder JM. Neuropathy with lysosomal changes in Marinesco-Sjogren syndrome: fine structural findings in skeletal muscle and conjunctiva. Neuropediatrics. 1992;23(6):329-35. doi: 10.1055/s-2008-1071368. PubMed PMID: 1491754.

205. Masiero E, Agatea L, Mammucari C, Blaauw B, Loro E, Komatsu M, et al. Autophagy Is Required to Maintain Muscle Mass. Cell metabolism. 2009;10(6):507-15. doi: https://doi.org/10.1016/j.cmet.2009.10.008.

206. Lecker SH, Jagoe RT, Gilbert A, Gomes M, Baracos V, Bailey J, et al. Multiple types of skeletal muscle atrophy involve a common program of changes in gene expression. Faseb j. 2004;18(1):39-51. Epub 2004/01/14. doi: 10.1096/fj.030610com. PubMed PMID: 14718385.

207. Edström E, Altun M, Hägglund M, Ulfhake B. Atrogin-1/MAFbx and MuRF1 Are Downregulated in Aging-Related Loss of Skeletal Muscle. The Journals of Gerontology: Series A. 2006;61(7):663-74. doi: 10.1093/gerona/61.7.663.

208. O’Neill BT, Lee KY, Klaus K, Softic S, Krumpoch MT, Fentz J, et al. Insulin and IGF-1 receptors regulate FoxO-mediated signaling in muscle proteostasis. The Journal of clinical investigation. 2016;126(9):3433-46. doi: 10.1172/JCI86522.

209. Bentzinger CF, Lin S, Romanino K, Castets P, Guridi M, Summermatter S, et al. Differential response of skeletal muscles to mTORC1 signaling during atrophy and hypertrophy. Skeletal muscle. 2013;3(1):6. doi: 10.1186/2044-5040-3-6.

210. Agoulnik IU, Hodgson MC, Bowden WA, Ittmann MM. INPP4B: the new kid on the PI3K block. Oncotarget. 2011;2(4):321-8. Epub 2011/04/14. doi: 10.18632/oncotarget.260. PubMed PMID: 21487159; PubMed Central PMCID: PMCPMC3248162.

211. Balakrishnan A, Chaillet JR. Role of the inositol polyphosphate-4-phosphatase type II Inpp4b in the generation of ovarian teratomas. Developmental Biology. 2013;373(1):118-29. doi: https://doi.org/10.1016/j.ydbio.2012.10.011.

212. Fridlyand LE, Phillipson LH. Mechanisms of glucose sensing in the pancreatic $\beta$ cell. Islets. 2011;3(5):224-30. doi: 10.4161/isl.3.5.16409.

213. Sanford T. The Role of BiP Nucleotide Exchange Factor Sill in Immunoglobulin Biosynthesis. 2012.

214. Bromberg MB, Junck L, Gebarski SS, McLean MJ, Gilman S. The marinescosjögren syndrome examined by computed tomography, magnetic resonance, and 
18f-2-fluoro-2-deoxy-d-glucose and positron emission tomography. Archives of neurology. 1990;47(11):1239-42. doi: 10.1001/archneur.1990.00530110101025.

215. Wang J, Takeuchi T, Tanaka S, Kubo SK, Kayo T, Lu D, et al. A mutation in the insulin 2 gene induces diabetes with severe pancreatic beta-cell dysfunction in the Mody mouse. The Journal of clinical investigation. 1999;103(1):27-37. Epub 1999/01/12. doi: 10.1172/jci4431. PubMed PMID: 9884331; PubMed Central PMCID: PMCPMC407861.

216. Ichhaporia VP, Sanford T, Howes J, Marion TN, Hendershot LM, Gilmore R. Sil1, a nucleotide exchange factor for BiP, is not required for antibody assembly or secretion. Molecular biology of the cell. 2015;26(3):420-9. doi: 10.1091/mbc.e14-09-1392. PubMed PMID: 25473114.

217. Wang J, Sevier CS. Formation and Reversibility of BiP Protein Cysteine Oxidation Facilitate Cell Survival during and post Oxidative Stress. Journal of Biological Chemistry. 2016;291(14):7541-57. doi: 10.1074/jbc.M115.694810.

218. Stevens KLP, Black AL, Wells KM, Yeo KYB, Steuart RFL, Stirling CJ, et al. Diminished Ost3-dependent N-glycosylation of the BiP nucleotide exchange factor Sil1 is an adaptive response to reductive ER stress. Proceedings of the National Academy of Sciences. 2017;114(47):12489-94. doi: 10.1073/pnas. 1705641114 .

219. Rosam M, Krader D, Nickels C, Hochmair J, Back KC, Agam G, et al. Bap (Sil1) regulates the molecular chaperone $\mathrm{BiP}$ by coupling release of nucleotide and substrate. Nature structural \& molecular biology. 2018;25(1):90-100. doi: 10.1038/s41594-017-0012-6.

220. Fujitake J, Komatsu Y, Hataya Y, Nishikawa A, Eriguchi M, Mizuta H, et al. A case of Marinesco-Sjogren syndrome: MRI observations of skeletal muscles, bone metabolism, and treatment with testosterone and risedronate. Internal medicine (Tokyo, Japan). 2011;50(2):145-9. Epub 2011/01/20. PubMed PMID: 21245640.

221. Rowe SM, Verkman AS. Cystic fibrosis transmembrane regulator correctors and potentiators. Cold Spring Harbor perspectives in medicine. 2013;3(7). Epub 2013/07/03. doi: 10.1101/cshperspect.a009761. PubMed PMID: 23818513; PubMed Central PMCID: PMCPMC3685879.

222. Kawahara G, Hayashi YK. Characterization of Zebrafish Models of MarinescoSjögren Syndrome. PLOS ONE. 2016;11(10):e0165563. doi: 10.1371/journal.pone.0165563.

223. Kashimada A, Hasegawa S, Isagai T, Uchiyama T, Matsuo M, Kawai M, et al. Targeting the enhanced ER stress response in Marinesco-Sjögren syndrome. Journal of the neurological sciences. 2018;385:49-56. doi: https://doi.org/10.1016/j.jns.2017.12.010. 
224. Bergmann TJ, Fregno I, Fumagalli F, Rinaldi A, Bertoni F, Boersema PJ, et al. Chemical stresses fail to mimic the unfolded protein response resulting from luminal load with unfolded polypeptides. Journal of Biological Chemistry. 2018;293(15):5600-12. doi: 10.1074/jbc.RA117.001484.

225. Kim AJ, Shi Y, Austin RC, Werstuck GH. Valproate protects cells from ER stress-induced lipid accumulation and apoptosis by inhibiting glycogen synthase kinase-3. Journal of cell science. 2005;118(1):89-99. doi: 10.1242/jcs.01562.

226. Das I, Png CW, Oancea I, Hasnain SZ, Lourie R, Proctor M, et al. Glucocorticoids alleviate intestinal ER stress by enhancing protein folding and degradation of misfolded proteins. The Journal of experimental medicine. 2013;210(6):1201-16. doi: 10.1084/jem.20121268.

227. Grande V, Ornaghi F, Comerio L, Restelli E, Masone A, Corbelli A, et al. PERK inhibition delays neurodegeneration and improves motor function in a mouse model of Marinesco-Sjögren syndrome. Human molecular genetics. 2018;27(14):2477-89. doi: 10.1093/hmg/ddy152.

228. Axten JM, Medina JR, Feng Y, Shu A, Romeril SP, Grant SW, et al. Discovery of 7-methyl-5-(1-\{[3-(trifluoromethyl)phenyl]acetyl\}-2,3-dihydro-1H-indol-5-yl)7H-p yrrolo[2,3-d]pyrimidin-4-amine (GSK2606414), a potent and selective firstin-class inhibitor of protein kinase R (PKR)-like endoplasmic reticulum kinase (PERK). Journal of medicinal chemistry. 2012;55(16):7193-207. Epub 2012/07/26. doi: 10.1021/jm300713s. PubMed PMID: 22827572.

229. Rojas-Rivera D, Delvaeye T, Roelandt R, Nerinckx W, Augustyns K, Vandenabeele P, et al. When PERK inhibitors turn out to be new potent RIPK1 inhibitors: critical issues on the specificity and use of GSK2606414 and GSK2656157. Cell Death Differ. 2017;24(6):1100-10. Epub 2017/04/30. doi: 10.1038/cdd.2017.58. PubMed PMID: 28452996; PubMed Central PMCID: PMCPMC5442476.

230. Das I, Krzyzosiak A, Schneider K, Wrabetz L, D'Antonio M, Barry N, et al. Preventing proteostasis diseases by selective inhibition of a phosphatase regulatory subunit. Science. 2015;348(6231):239-42. Epub 2015/04/11. doi: 10.1126/science.aaa4484. PubMed PMID: 25859045; PubMed Central PMCID: PMCPMC4490275.

231. Krzyzosiak A, Sigurdardottir A, Luh L, Carrara M, Das I, Schneider K, et al. Target-Based Discovery of an Inhibitor of the Regulatory Phosphatase PPP1R15B. Cell. 2018. doi: https://doi.org/10.1016/j.cell.2018.06.030.

232. Liu Z-C, Chu J, Lin L, Song J, Ning L-N, Luo H-B, et al. SIL1 Rescued Bip Elevation-Related Tau Hyperphosphorylation in ER Stress. Molecular neurobiology. 2016;53(2):983-94. doi: 10.1007/s12035-014-9039-4. 
233. Labisch T, Buchkremer S, Phan V, Kollipara L, Gatz C, Lentz C, et al. Tracking Effects of SIL1 Increase: Taking a Closer Look Beyond the Consequences of Elevated Expression Level. Molecular neurobiology. 2018;55(3):2524-46. doi: 10.1007/s12035-017-0494-6.

234. Xu H, Xu S, Zhang R, Xin T, Pang Q. SIL1 functions as an oncogene in glioma by AKT/mTOR signaling pathway. OncoTargets and therapy. 2018;11:3775-83. Epub 2018/07/13. doi: 10.2147/ott.S167552. PubMed PMID: 29997438; PubMed Central PMCID: PMCPMC6033116. 
A

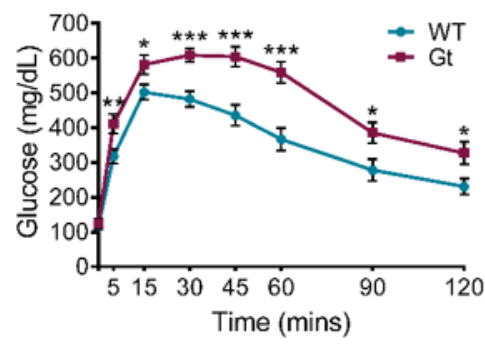

C

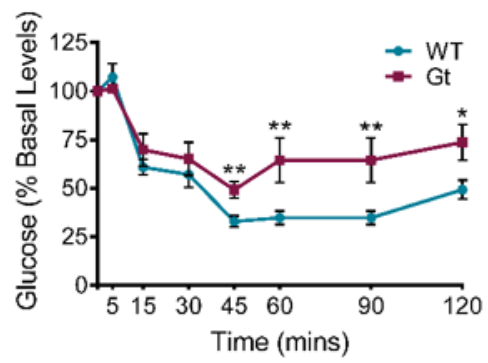

B

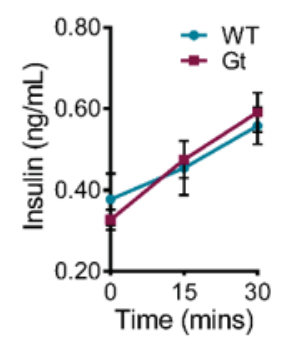

Figure A-1. $\quad$ Sil1 $^{\mathrm{Gt}}$ mice demonstrate glucose intolerance and insulin resistance.

Age-matched wild-type and Sill ${ }^{\mathrm{Gt}}$ mice $(n=8-12)$ were subjected to an (A) intraperitoneal Glucose Tolerance Test (i.p.GTT), or (C) an intraperitoneal Insulin Tolerance Test (i.p.ITT). Area Under Curve (AUC) calculations for each test is displayed next to the respective blood glucose levels. Error bars indicate means \pm s.e.m. (B) Plasma insulin levels during the initial time-course of the i.p.GTT were measured and plotted. Statistical comparisons were performed using t-tests; P-values are indicated as $* P \leq 0.05$, $* * P \leq 0.01$, $* * * P \leq 0.001, * * * * P \leq 0.0001$. 
A

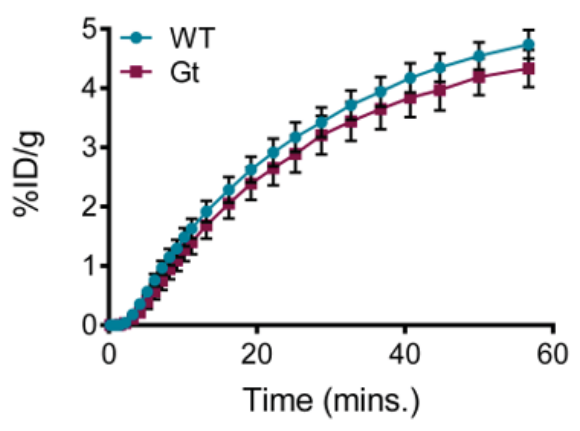

C

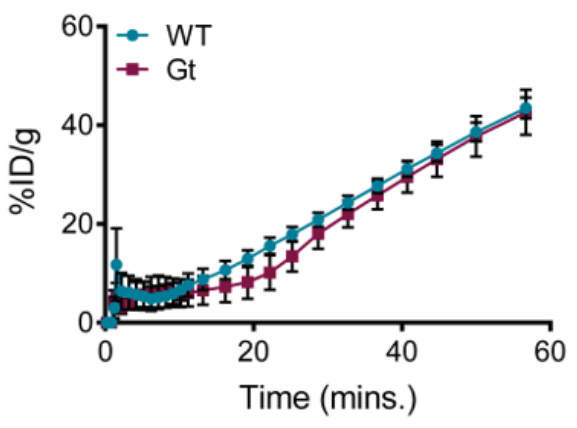

B

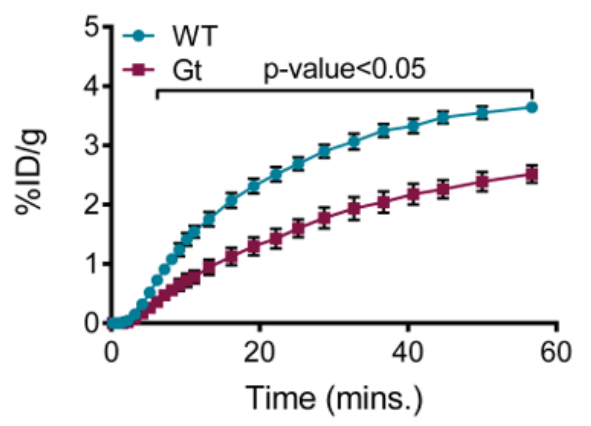

D

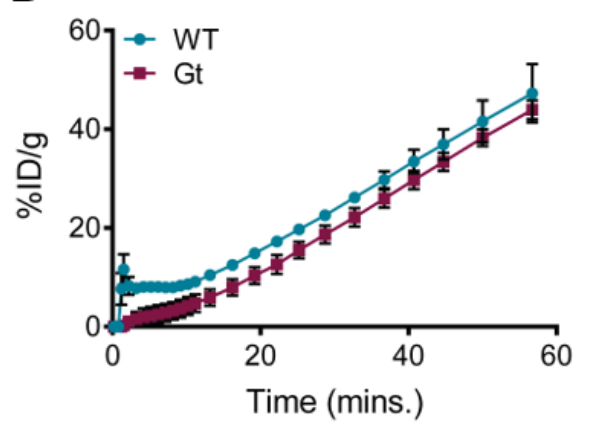

Figure A-2. Loss of SIL1 leads to defects in brain glucose uptake in Sil1 ${ }^{\mathrm{Gt}}$ mice.

Graphical representation of ${ }^{18} \mathrm{~F}$-fluorodeoxyglucose uptake over 60 minutes in wild-type (blue) and $\mathrm{Sill}^{\mathrm{Gt}}$ (red) brains (A, B) and urinary bladders (C, D) under basal conditions $(A, C)$ or after an intraperitoneal injection of $0.75 \mathrm{mU} / \mathrm{g}$ body weight of human insulin (B, D; $n=4$ ) assayed by PET- $\mu$ CT. Statistical differences were computed using unpaired, two-tailed Student's $t$-tests, and are indicated in the graph. $\% \mathrm{ID} / \mathrm{g}, \%$ injected dose per gram body weight. 

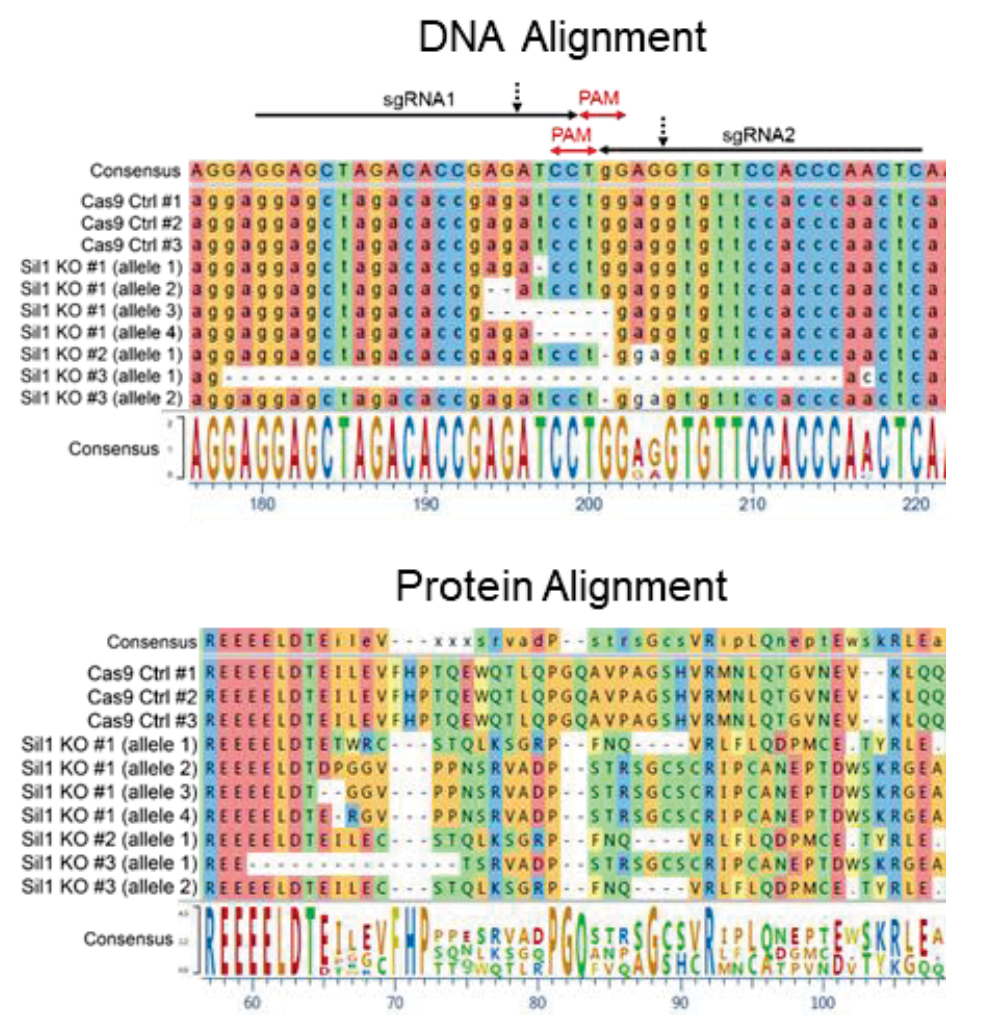

Figure A-3. Deep sequencing reveals successful CRISPR/Cas9-mediated targeting of the Sil1 locus in murine C2C12 myoblasts.

SgRNA sequences targeting exon 3 of the murine Sill gene were 5'GGAGCTAGACACCGAGATCC-3' (sgRNA \#1) and 5'-

GAGTTGGGTGGAACACCTCC-3' (sgRNA \#2), respectively, and were designed to recapitulate the MSS-associated p.E60X mutation (86). DNA oligonucleotides containing sgRNA sequences were cloned into a PX458 (SpCas9-2A-EGFP-U6-sgRNA, Addgene 48138) all-in-one backbone using the Bbs1 restriction enzyme site. Clones were verified by Sanger sequencing using the U6 promoter forward primer 5'-

GAGGGCCTATTTCCCATGAT-3'. The PX458 all-in-one vector containing either sgRNA \#1 or \#2, respectively, or the vector alone (experimental control) were transfected into the polyploid C2C12 murine skeletal muscle myoblasts (ATCC $\left(\mathrm{CRL}-1772^{\mathrm{TM}}\right.$ ) using Lipofectamine ${ }^{\mathrm{TM}} 3000$, as per the company protocol. 24 hours following transfection, GFP-expressing cells by were sorted by FACS, allowed to recover in tissue culture and subsequently subjected to single-cell sorting by FACS. The Exon 3 region of

Sill from single cell-derived clonal populations was amplified using forward (5'-

CAGTGCTAACCCTCTCCCTAGTATTTTCTTTAACC-3') and reverse (5'-

GAGCCAAGCAGCAGAGCCCAAAATAGC-3') primers and subjected to deep sequencing to characterize the Sill mutations. DNA and protein alignments of three Cas9 control and Sill-targeted single-cell clones displayed above demonstrate efficient locus targeting, which generated frameshift mutations that cause premature truncations of the encoded SIL1 protein. 
A

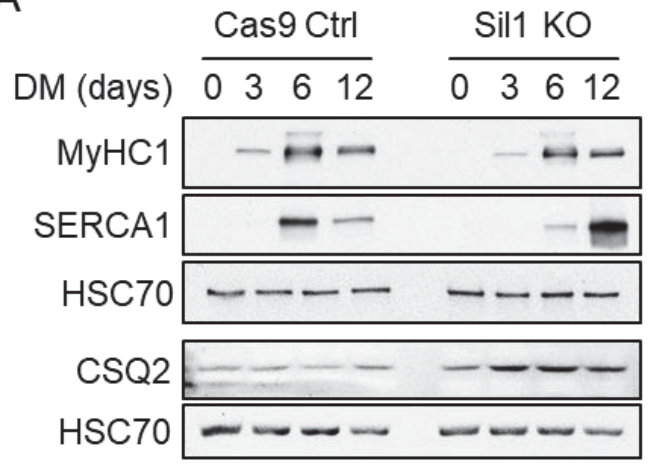

B

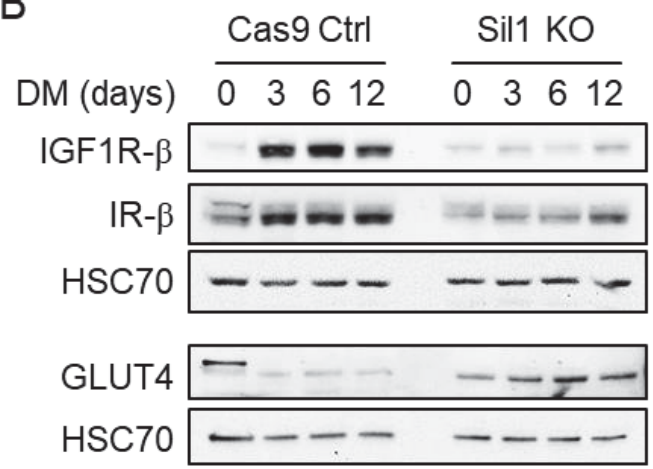

Figure A-4. SIL1-null myoblasts retain the ability to differentiate into myotubes and demonstrate molecular changes similar to that observed in Sill $^{\mathrm{Gt}}$ skeletal muscles.

Preliminary western blot analysis of CRISPR/Cas9-engineered SIL1-null and control myoblasts revealed that both had similar growth rates and differentiated into myotubes that express myosin heavy chain with similar kinetics (A), confirming that SIL1 is dispensable for myotube differentiation. Similar to $\mathrm{Sill}^{\mathrm{Gt}}$ quadriceps (Figure 3-13C), both IR- $\beta$ and IGF1R- $\beta$ were decreased and GLUT4 was increased (B), arguing that CRISPR/Cas9-engineered SIL1-knockout myoblasts provide a useful tool for assessing ER protein folding and quality control pathways, which are critical to muscle function. This also was true for calsequestrin 2 levels (Figure 3-6A). 


\section{VITA}

Viraj Ichhaporia, born in 1988, graduated high school from Kishinchand Chellaram College, India, in May of 2006. He obtained his Bachelor of Science (Hons.) degree from the University of Mumbai, India, with a dual major in Life Sciences and Biochemistry in 2009. He then enrolled at the Maharaja Sayajirao University of Baroda, Vadodara, India, and completed his Master of Science degree with a major in Medical Biotechnology in 2011. He enrolled at the University of Tennessee Health Science Center in August of 2011 as a Ph.D. student and has been conducting his doctoral research on understanding the molecular mechanisms of Marinesco-Sjögren Syndrome. He is expected to obtain his Ph.D. degree in December 2018.

\section{Publications:}

Ichhaporia, V.P., Sanford, T., Howes, J., Marion, T.N. and Hendershot, L.M., 2015. Sil1, a nucleotide exchange factor for $\mathrm{BiP}$, is not required for antibody assembly or secretion. Molecular biology of the cell, 26(3), pp.420-429.

Ichhaporia, V.P., Kim, J., Kavdia, K., Vogel, P., Horner, L., Frase, S. and Hendershot, L.M., 2018. SIL1, the endoplasmic-reticulum-localized BiP co-chaperone, plays a crucial role in maintaining skeletal muscle proteostasis and physiology. Disease Models \& Mechanisms, 11(5), p.dmm033043.

Bai, B., Tan, H., Pagala, V.R., High, A.A., Ichhaporia, V.P., Hendershot, L. and Peng, J., 2017. Deep profiling of proteome and phosphoproteome by isobaric labeling, extensive liquid chromatography, and mass spectrometry. In Methods in enzymology (Vol. 585, pp. 377-395). Academic Press.

Wang, B., Joo, J.H., Mount, R., Teubner, B.J., Krenzer, A., Ward, A.L., Ichhaporia, V.P., Adams, E.J., Khoriaty, R., Peters, S.T. and Pruett-Miller, S.M., 2018. The COPII cargo adapter SEC24C is essential for neuronal homeostasis. The Journal of clinical investigation, 128(8). 